\title{
Quantification of soluble organic fraction measurement variation in diesel particulate matter emissions
}

John Wesley Hardin

West Virginia University

Follow this and additional works at: https://researchrepository.wvu.edu/etd

\section{Recommended Citation}

Hardin, John Wesley, "Quantification of soluble organic fraction measurement variation in diesel particulate matter emissions" (2008). Graduate Theses, Dissertations, and Problem Reports. 1974. https://researchrepository.wvu.edu/etd/1974

This Thesis is protected by copyright and/or related rights. It has been brought to you by the The Research Repository @ WVU with permission from the rights-holder(s). You are free to use this Thesis in any way that is permitted by the copyright and related rights legislation that applies to your use. For other uses you must obtain permission from the rights-holder(s) directly, unless additional rights are indicated by a Creative Commons license in the record and/ or on the work itself. This Thesis has been accepted for inclusion in WVU Graduate Theses, Dissertations, and Problem Reports collection by an authorized administrator of The Research Repository @ WVU. For more information, please contact researchrepository@mail.wvu.edu. 


\title{
Quantification of Soluble Organic Fraction Measurement Variation in Diesel
} Particulate Matter Emissions

\author{
John Wesley Hardin \\ Thesis submitted to the \\ College of Engineering and Mineral Resources \\ at West Virginia University \\ in partial fulfillment of the requirements \\ for the degree of \\ Master of Science \\ in \\ Mechanical Engineering \\ Gregory J. Thompson, Ph.D., Chair \\ Nigel N. Clark, Ph.D. \\ Mridul Gautam, Ph.D. \\ Department of Mechanical Engineering \\ Morgantown, West Virginia \\ 2008
}

Keywords: Diesel Engines, Emissions, Soluble Organic Fraction. 


\title{
ABSTRACT \\ Quantification of Soluble Organic Fraction Measurement Variation in Diesel Particulate Matter Emissions
}

\author{
John Wesley Hardin
}

\begin{abstract}
Heavy-duty diesel engines (HDDE) are the dominate prime movers of goods and services in the United States (US) and the world today. HDDE will continue to lead the transportation sector in the near term because of its superior fuel economy and performance compared to existing alternatives. However, HDDE produce many harmful emissions constituents that include: hydrocarbons (HC), oxides of nitrogen (NOx), carbon monoxide (CO), and particulate matter (PM). Due to these harmful emissions, the US Environmental Protection Agency (EPA) has been entrusted to enforce the Clean Air Act to limit the amount of emissions that can be produced from these HDDE. Additionally, the EPA requires that each state maintain a state implantation plan (SIP) to ensure minimum air quality standards. Because of SIP concerns, California and Texas have identified on-road heavy-duty diesel engines as a significant pollution source and have implemented an alternative diesel fuel formulation program in order to meet their SIP. To meet these more stringent fuel requirements, refineries use additives in diesel fuel to reduce these emissions. One main concern is when reducing these emissions using fuel additives, PM may be increased. PM is composed of soluble organics along with other constituents. The soluble organic fraction (SOF) contains polynuclear (or polycyclic) aromatic hydrocarbons (PAH) and nitro-PAHs (nitrogen-bound $\mathrm{PAH}$ ) that are carcinogens and linked to heart and respiratory diseases. Because of this, California has required that the SOF emissions from HDDE fueled with on-road fuels sold in the state of California must not increase SOF more than $6 \%$ above a 48 cetane, $10 \%$ aromatic reference fuel SOF emissions values.
\end{abstract}

The purpose of this research was to develop a test procedure for SOF determination and quantifying the variability when using Soxhlet extraction. This was accomplished by extracting SOF from PM gathered from engines exercised over an engine dynamometer transient test cycle using various fuels. Also, the filter media, loaded and unloaded with PM, was examined to understand weighing variations over time in a controlled environment. Lastly, handling issues with the transportation of the filter media was examined.

The test procedure developed for SOF determination produced a coefficient of variation of $4.3 \%$. Weighing variations were found to be caused from constituents lost by the filter media during the extraction process. However, some of this weight loss was then regained while the filter was conditioning in a conditioned clean room. This weight gain can be expressed using the equation: $\mathrm{W}_{\text {gain }}=.001175\left(\mathrm{t}_{\text {hours }}{ }^{0.74014}\right)$. Lastly, shipping and receiving of filter media caused slight weight loss of initial weighing before extraction but had no affect on weight gain after extraction. 


\section{ACKNOWLEDGMENTS}

It has been along journey to get to the point I am today, and with that, there are many people I want to give thanks to:

First and foremost, my advisor, Dr. Thompson for giving me a job at the ERC, without this I wouldn't have a thesis to work on, also for Dr. Thompson allowing me to take a job in the middle of my thesis work, but continue to work with me in the afternoons to finish my thesis. All of the employees at the ERC who worked with me to gather my research, especially Brad Ralston, who was patient enough to teach me about the laboratory when I knew he was way to busy to,

To Geoff Newlin and Moy Dam for assisting in weighing filters,

All my friends I made along the way in college including Mike Ursic, Kevin Flaim, and Ray Tincher, who sat with me in the test cell all those weekends performing research and running the test bench.

Aurora Flight Sciences for working with me to finish classes, research and my thesis, my parents, Ron and Chele Hardin, and my sister Cari Baliles, for pushing me to finish my thesis, when I didn't think it would be possible.

Lastly, my wonderful girlfriend, Meredith Jenkins, who I would be lost without during my last hectic year and a half of college and a fulltime job.

Without all these people, it would not have been possible to be graduating with a Masters in Mechanical Engineering, I Thank You... 


\section{Table of Contents}

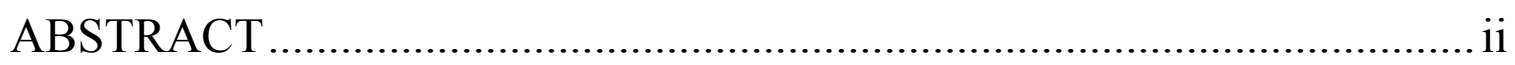

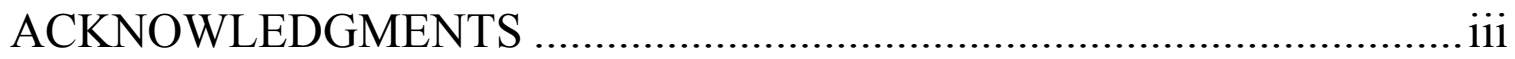

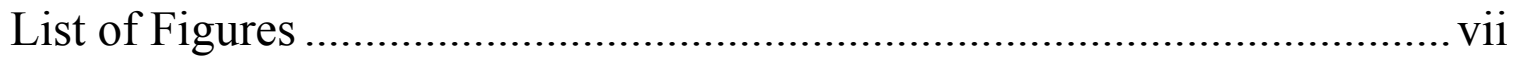

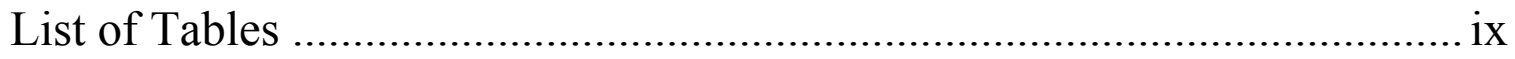

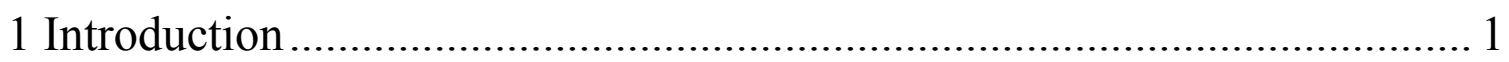

1.1 Prologue

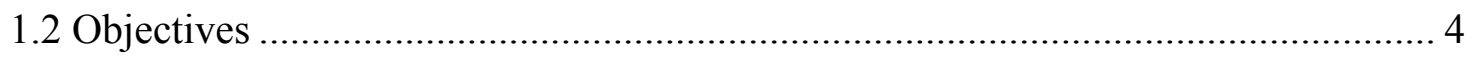

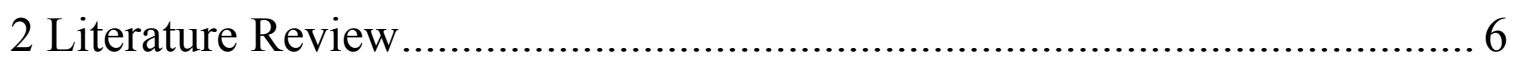

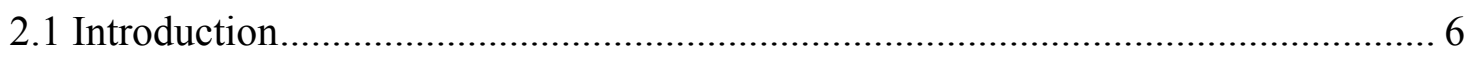

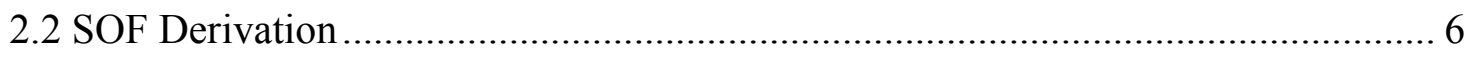

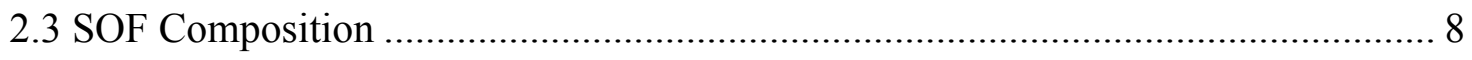

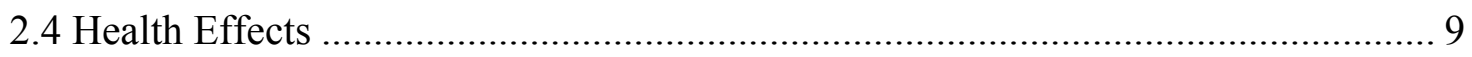

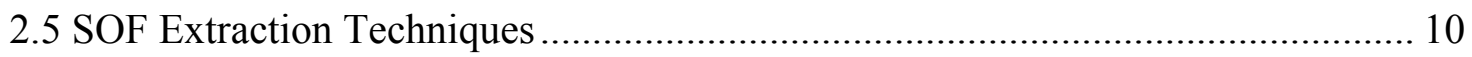

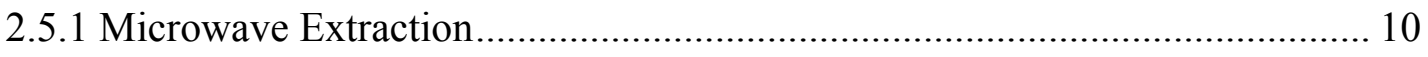

2.5.2 Supercritical Extraction............................................................................ 10

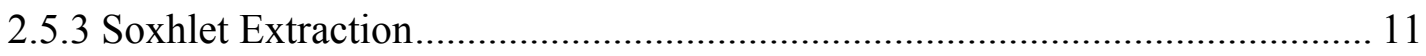

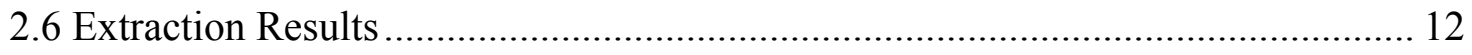

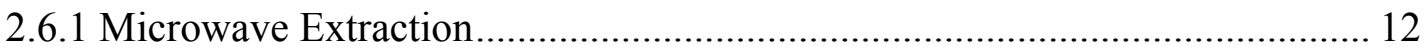

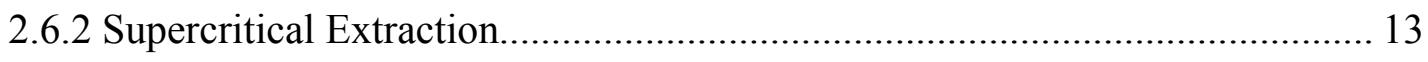

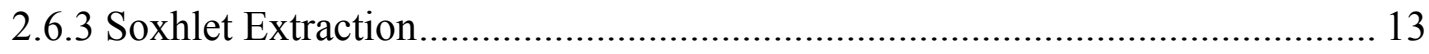


3 Experimental Procedures and Equipment................................................ 15

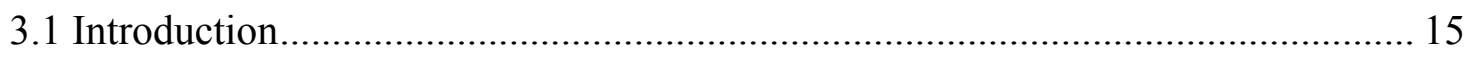

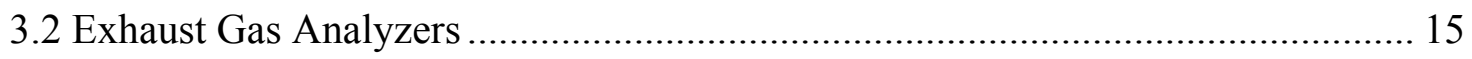

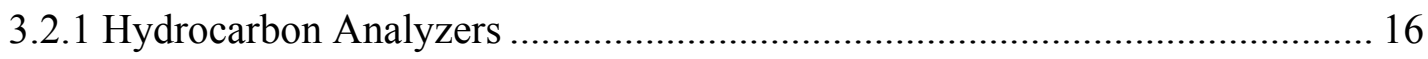

3.2.1 Oxides of Nitrogen (NOx) Analyzer ........................................................... 17

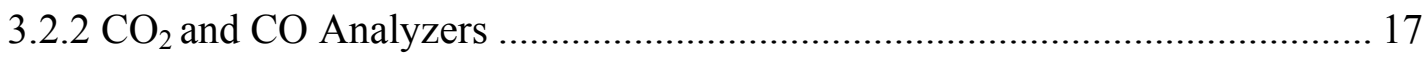

3.3 Full Flow Exhaust Dilution Tunnel ................................................................... 17

3.4 Particulate Matter Sampling System............................................................... 20

3.5 Clean Room Weighing and Filter Conditioning ……........................................ 21

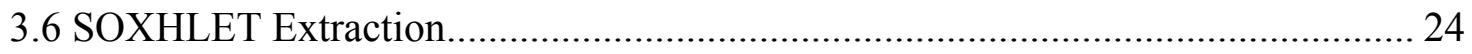

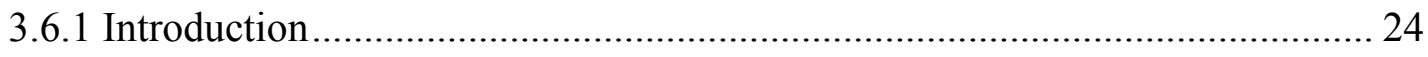

3.6.2 Soxhlet Laboratory Setup ....................................................................... 24

3.6.3 SOXHLET Extraction Procedure.............................................................. 25

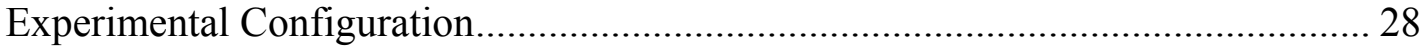

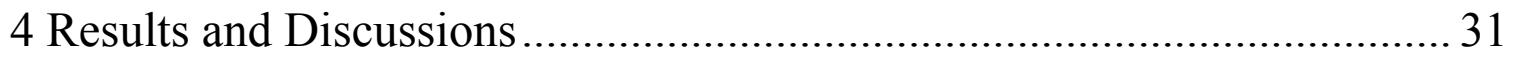

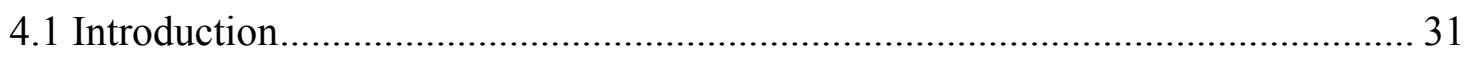

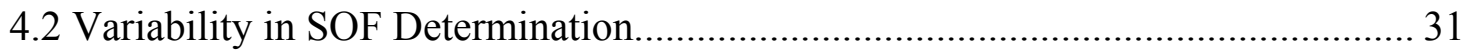

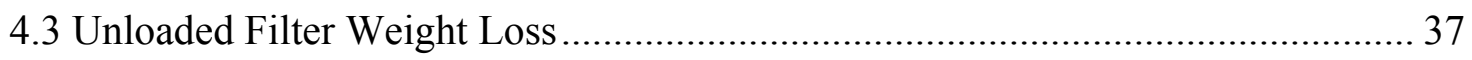

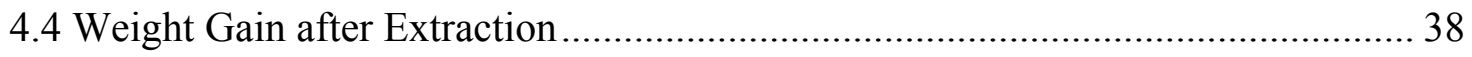

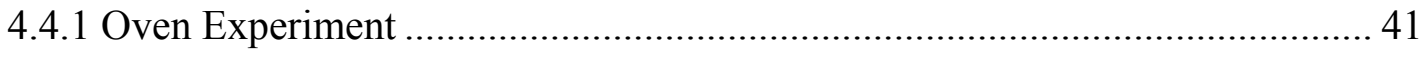

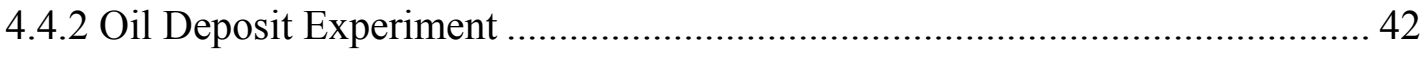

4.4.5 Loaded vs. Non Loaded Filters ........................................................................ 43

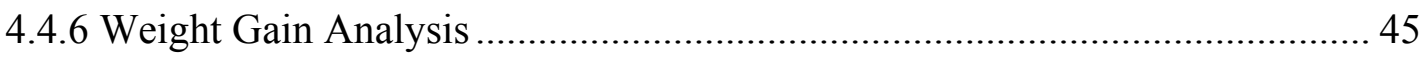


4.5 Handling of Filter Media .............................................................................. 46

5 Conclusions and Recommendations ............................................... 49

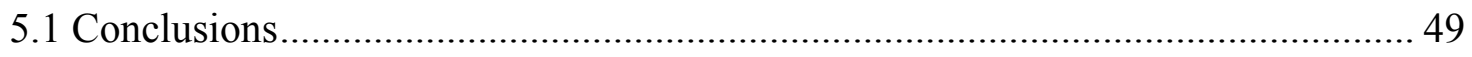

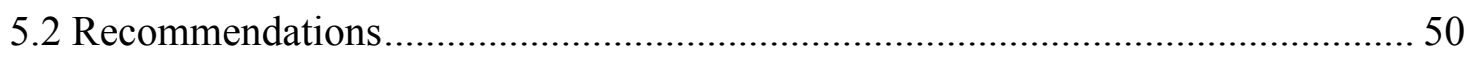

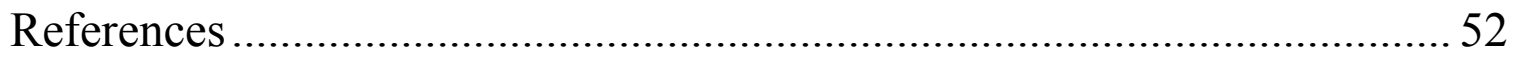

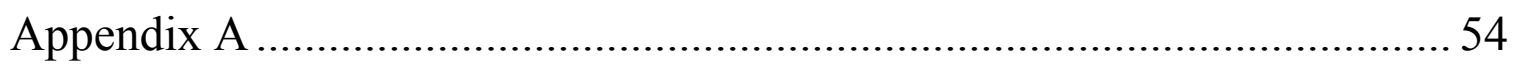

Appendix B ........................................................................... 57

Appendix C ................................................................................ 59

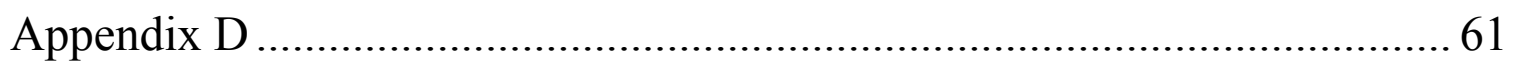

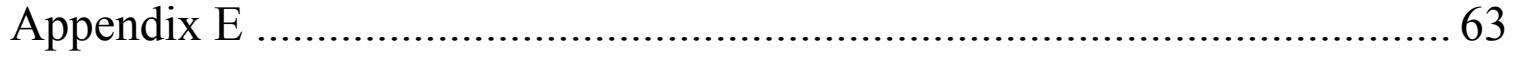

Appendix F .......................................................................... 70 


\section{List of Figures}

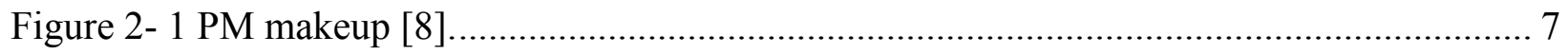

Figure 2- 2 Engine load and Exhaust Temperature effects on SOF formation [7] ..................... 8

Figure 2- 3 A chromatograph of a SOF sample [8] ..................................................... 9

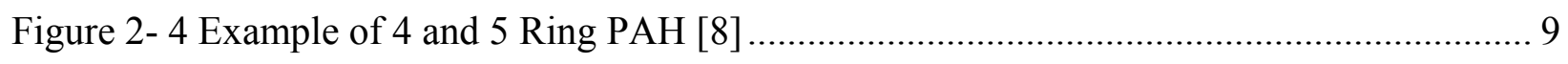

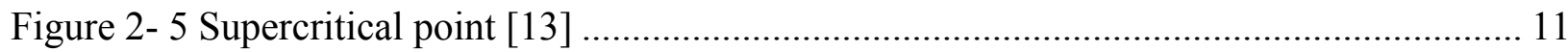

Figure 2- 7 Results of factorial design in microwave extraction of SOF in diesel PM [10] ....... 13

Figure 2- 8 Results of factorial design in Soxhlet extraction of SOF in diesel PM [10] ............ 14

Figure 3- 1 Exhaust Analyzer Bench ........................................................................... 16

Figure 3- 2 Full Scale Dilution Tunnel ............................................................................. 18

Figure 3- 3 Secondary Dilution Tunnel with Filter Compartment ......................................... 19

Figure 3- 4 Schematic of Full Scale and Secondary Dilution Tunnels................................... 20

Figure 3- 5 Stainless steel filter holder connected in line with the Secondary Dilution Tunnel .. 21

Figure 3- 6 Stainless steel filter housing separated into it's components............................... 21

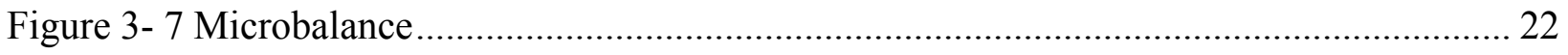

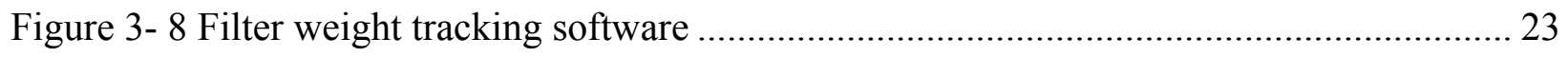

Figure 3- 9 Extraction Apparatus, Heating Mantle, and Heat Control Module......................... 25

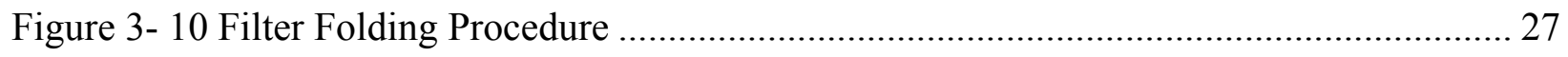

Figure 3- 11 Thimble with filter inserted into extractor ................................................ 28

Figure 4- 1 Allowable SOF Variation for Passage ................................................................ 34

Figure 4- 2 COV of SOF for Each Set of FTPs PM filters Extracted .................................... 36

Figure 4- 3 Net weight loss of an unloaded filter due to Soxhlet extraction ........................... 38

Figure 4- 4 Weighed Gained after Extraction over Time ...................................................... 39 
Figure 4- 5 Reference Filter Tracking ................................................................................ 40

Figure 4- 6 Weight Gain of Unused T60A20 Extracted Filters Over 31 Days.............................. 41

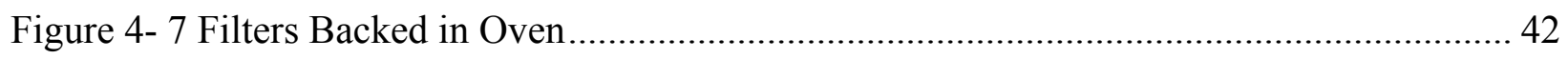

Figure 4- 8 Oil Deposited Filters ………………………................................................... 43

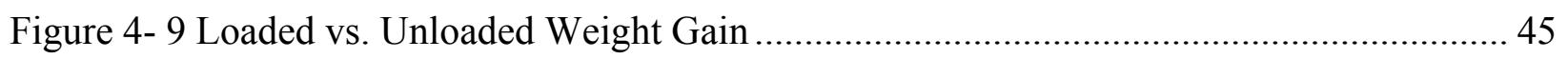

Figure 4- 10 Weight Gained after Extraction over Time ............................................................... 46

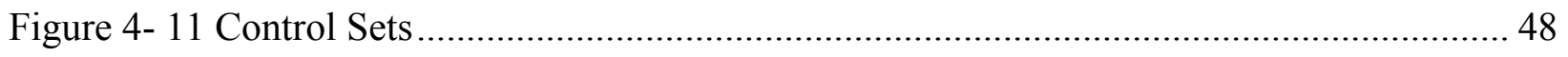




\section{List of Tables}

Table 1-1 EPA Heavy-Duty Diesel Engine Emissions Standards (g/bhp-hr) [2] ...................... 1

Table 1-2 Fuel property requirements for on-road fuels [6] .............................................. 3

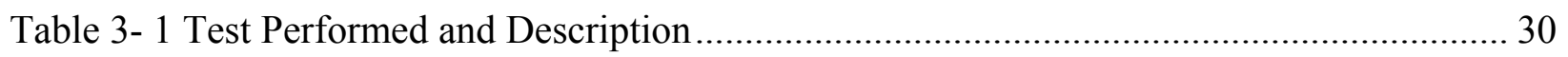




\section{Introduction}

\subsection{Prologue}

Heavy-duty diesel engines (HDDE) are the dominate prime movers of goods and services in the United States (US) and the world today and will continue to lead the transportation sector in the near term because of its superior fuel economy and performance compared to existing alternatives. However, HDDE produce many harmful emissions constituents that include: hydrocarbons $(\mathrm{HC})$, oxides of nitrogen $(\mathrm{NOx})$, carbon monoxide $(\mathrm{CO})$, and particulate matter (PM). Additionally, $\mathrm{HC}$ are divided between methane and non-methane hydrocarbons (NMHC) because of green house gas reactivity concerns. Due to these harmful emissions, the US Environmental Protection Agency (EPA) has been entrusted to enforce the Clean Air Act [1] to limit the amount of emissions that can be produced from these HDDE as shown in Table 1-1. As illustrated in this table, NOx and PM emissions will have been reduced by over $98 \%$ between 1988 and 2010.

Table 1-1 EPA Heavy-Duty Diesel Engine Emissions Standards (g/bhp-hr) [2]

\begin{tabular}{|l|c|c|c|c|c|c|}
\hline Year & HC & CO & NOx & PM & NMHC + NOx & NMHC \\
\hline 1988 & 1.3 & 15.5 & 10.7 & 0.6 & n/a & n/a \\
\hline 1990 & 1.3 & 15.5 & 6.0 & 0.6 & n/a & n/a \\
\hline 1991 & 1.3 & 15.5 & 5.0 & 0.25 & n/a & n/a \\
\hline 1994 & 1.3 & 15.5 & 5.0 & 0.1 & n/a & n/a \\
\hline 1998 & 1.3 & 15.5 & 4.0 & 0.1 & n/a & n/a \\
\hline $2004 *$ (option 1) & 1.3 & 15.5 & n/a & 0.1 & 2.4 & n/a \\
\hline $2004 *$ (option 2) & 1.3 & 15.5 & n/a & 0.1 & 2.5 & 0.5 \\
\hline $2007-2010$ & 1.3 & 15.5 & 0.2 & 0.01 & n/a & 0.14 \\
\hline
\end{tabular}

* 2004 was moved to October 2002

Although the emissions of NOx and PM from HDDE have been reduced by two orders of magnitude over the last two decades, some cities and states still have trouble meeting minimum air quality standards. Specifically, states such as California and Texas have difficulties meeting their state implementation plans (SIP) on certain criteria pollutants. As such, these states have 
implemented measures to reduce those pollutants that are in violation of their respected SIP. With regards to HDDE, California and Texas have identified that by changing the diesel fuel composition, lower emissions can be achieved and thus reduce the ambient emissions that are a concern in their SIP. Although diesel fuel sold in the US must meet certain standards for emissions and engine performance as shown in Table 1-2, California and Texas have placed tighter requirements on fuels that are to be sold in these states. Specifically, a minimum cetane number of 48 and a maximum aromatic content of $10 \%$ is specified to be sold in these two states. It is well documented that increased cetane number and decreased aromatic content will reduce NOx and PM emissions [3]. However, it is expensive for refineries to produce diesel fuels that are 48 cetane number and $10 \%$ aromatic content. Any additional cost that would be incurred by the refinery would be passed on to the consumer. A significant additional cost to diesel fuel would offset the fuel economy benefit that HDDE have over their spark ignition (gasoline) counterparts and would result in added costs to the consumer. Because of these additional costs, the states of California and Texas have implemented an alternative diesel fuel formulation program to demonstrate equivalency between a candidate fuel and a 48 cetane number and $10 \%$ aromatic reference fuel. The candidate fuel still has to meet the on-road specifications as shown in Table 1-2 but can be below the 48 cetane number and above the $10 \%$ aromatic content $[2,3]$. 
Table 1-2 Fuel property requirements for on-road fuels [6]

\begin{tabular}{|l|c|c|c|}
\hline Property & Test Method & Units & ASTM D975 \\
\hline Flash Point, min & D93 & ${ }^{\circ} \mathrm{F}$ & 125.6 \\
\hline Water and Sediment, max & D2709 & $\%$ vol & 0.05 \\
\hline Distillation Temperature @ 90\% & D86 & ${ }^{\circ} \mathrm{F}$ & $540-640$ \\
\hline Kinematic Viscosity at $40{ }^{\circ} \mathrm{C}$ & D445 & $\mathrm{mm}^{2} / \mathrm{s}$ & $1.9-4.1$ \\
\hline Ash, max & D482 & $\%$ mass & 0.01 \\
\hline Sulfur, max & D5453 & $\mathrm{ppm}$ mass & 15 \\
\hline Copper Strip Corrosion, max & D130 & - & No. 3 \\
\hline Cetane Number, min & D613 & - & 40 \\
\hline Aromaticity, max & D1319 & $\%$ vol & 35 \\
\hline Cloud Point, max & D2500 & ${ }^{\circ} \mathrm{C}$ & Report \\
\hline $\begin{array}{l}\text { Ramsbottom Carbon Residue on } \\
10 \% \text { distillation Residue, max }\end{array}$ & D524 & $\%$ mass & 0.35 \\
\hline Lubricity, HFRR at $60{ }^{\circ} \mathrm{C}, \max$ & D6079 & micron & 520 \\
\hline
\end{tabular}

Many refineries have been able to demonstrate equivalency of diesel product produced through refining processes. Many of these processes involve reducing the aromatic content of the diesel range with some form of hydrotreating to a level that has equivalent emissions as the reference fuel. Hydrotreating or hydrodesulfurization, uses a chemical process to remove sulfur and nitrogen from gasoline, jet fuels, and diesel fuels by vaporizing the fuel in the presence of hydrogen and introducing the mixture to a catalyst (for example, molybdenum disulfide). This catalyst causes a chemical reaction to occur, where sulfur and nitrogen compounds are converted to hydrogen sulfide and ammonia, then condensed and the hydrocarbons are separated from the gas [7]. However, as with any process, reducing the aromatic content through hydrotreating is relatively expensive. Another means to show equivalency by some has been through the use of additives to reduce the emissions from a higher aromatic diesel stock. Many of these additives increase the cetane number of the fuel without influencing the other fuel properties to any significant extent. The increased cetane number correlates to a shorter ignition delay which 
results in a lower NOx value and increase in PM [5]. The increase in PM is problematic in the California and Texas fuel equivalency tests since there is a limit to how high the candidate NOx and PM values can be relative to the reference fuel values. Presently, the candidate fuel NOx and PM values can be a nominal $1 \%$ and $2 \%$, respectively, higher than the reference fuel values. Additionally, California requires the reporting of soluble organic fraction (SOF) of the PM where the candidate fuel SOF value can only be $6 \%$ higher than the reference fuel SOF value.

Particulate matter is composed of three different matters: solid fraction (SOL), SOF, and sulfate particulates $\left(\mathrm{SO}_{4}\right)$. Of these three parts, $\mathrm{SOF}$ is the main concern. SOF is composed of unburned fuels, burned fuels and lubricating oils [8]. SOF also contains polynuclear (or polycyclic) aromatic hydrocarbons (PAH) and nitro-PAHs (nitrogen-bound PAH). These PAHs are carcinogens that are linked to heart and reparatory diseases [9]. Due to this, the California Air Resources Board (CARB) has enforced testing these candidate fuels to insure they do not cause an overall rise in SOF while trying to reduce other emissions.

The method for SOF determination is an extraction process known as Soxhlet. Soxhlet is a chemical process where solvent is cycled through a PM-loaded filter to dissolve all organic matter in the PM and only leaving the inorganic matter on the filter media. From this, the SOF can be determined from pre- and post-weight of the PM-loaded filter and from the post Soxhlet process filter weight.

\subsection{Objectives}

The main objective of this study was to develop a test procedure for SOF determination and quantifying the variability when using Soxhlet extraction. This was accomplished by extracting SOF from PM gathered from engines exercised over an engine dynamometer transient test cycles using various fuels. The second objective of this study was to examine how the SOF 
may vary over time when weighed in a controlled environment. This determined what weighing precautions must be taken to insure accurate results. The third and final objective of this work was to explore handling issues with the transportation of the filter media. 


\section{Literature Review}

\subsection{Introduction}

Diesel engines are known for excellent fuel economy and low CO and $\mathrm{HC}$ emissions, but with that come at the expense of high NOx and PM emissions relative to their gasoline counterparts. PM is formed from carbonaceous particulate that is comprised of inorganic and organic compounds [10]. The organic compounds are formed from engine lubrication oils, unburned fuel, PAH and nitro-PAHs [8]. These PAHs are highly carcinogenetic and also known to increase the risk of heart and respiratory diseases [9]. For this reason SOF is monitored from PM formed from diesel combustion. SOF is determined through an extraction process of a PM sample. In this section three different extraction processes will be explained and compared: microwave, supercritical, and Soxhlet along with SOF derivation, composition and health effects. Although present (2007 and newer model year) on-road HDDE's implement some form of particulate traps in the exhaust and hence change the PM mass and type coming out of the exhaust, this discussion will only focus on engine-out PM.

\subsection{SOF Derivation}

In pre-2007 HDDE regulations, diesel emission "particulates" are measured as any material deposited on the filter from the dilute exhaust gases sampled at a temperature lower than $125^{\circ} \mathrm{F}$ but above $68^{\circ} \mathrm{F}$ [9]. These particulate are made up of three different matters: solid fraction (SOL), SOF, and sulfate particulates $\left(\mathrm{SO}_{4}\right)$. SOL is composed of elemental carbon and ash, whereas $\mathrm{SO}_{4}$ is composed of sulfuric acid and water. One reason PM is so heavily restricted is SOF is derived from engine lubricating oil, unburned fuel and species formed from partially burned fuel [1]. As shown in Figure 2- 1, SOF makes up on average 32\% of the engine-out PM 
sample, where over two-thirds comes from the engine lube oil [8]. SOF formation depends highly on operating conditions of the engine such as test modes and duty cycles.

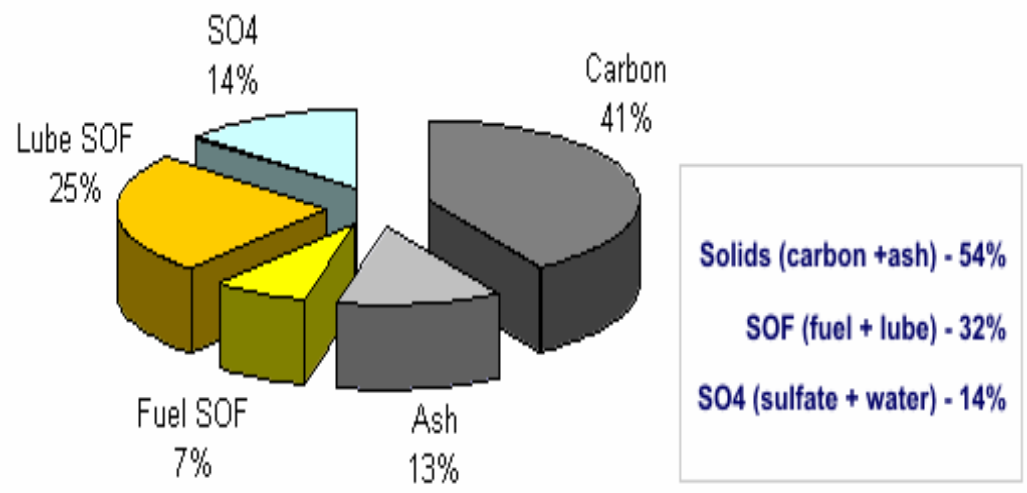

Figure 2- 1 PM makeup [8]

For some engines the SOF may only constitute $10 \%$ or less of the total PM deposited, this is known as a "dry" sample. Other engines may have a SOF of over $50 \%$, up to $80 \%$, and this is referred to as a "wet" sample. SOF formation is mainly affected by engine load and exhaust temperature. Figure 2- 2 shows as engine load and exhaust temperature increases SOF formation decreases. At low engine load and with temperatures lower then $100^{\circ} \mathrm{C} \mathrm{SOF}$ is at almost $50 \%$ of $\mathrm{PM}$, but as the engine reaches full load and temperatures greater then $400^{\circ} \mathrm{C}$ SOF drops to less than $5 \%$. 


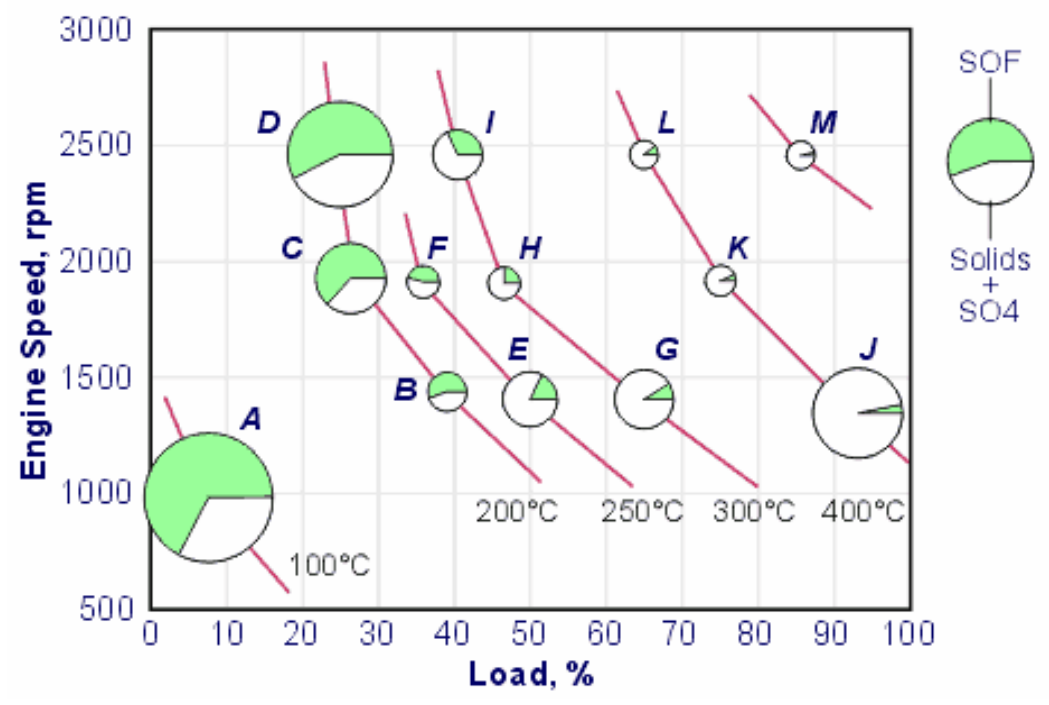

Figure 2- 2 Engine load and Exhaust Temperature effects on SOF formation [7]

\subsection{SOF Composition}

SOF is mainly composed of engine lube oil derived hydrocarbons, and some

hydrocarbons that come from high temperature boiling end fuel hydrocarbons. Diesel lube contains hydrocarbons that range from $\mathrm{C} 18$ to $\mathrm{C} 36$ whereas diesel fuel contains hydrocarbons from $\mathrm{C} 12$ to $\mathrm{C} 20$ [8]. An analysis of an SOF sample with a chromatograph illustrate that the majority of the hydrocarbons contain mostly hydrocarbon ranging from $\mathrm{C} 20$ to $\mathrm{C} 32$ corresponding with engine lube oil as shown in Figure 2- 3. 


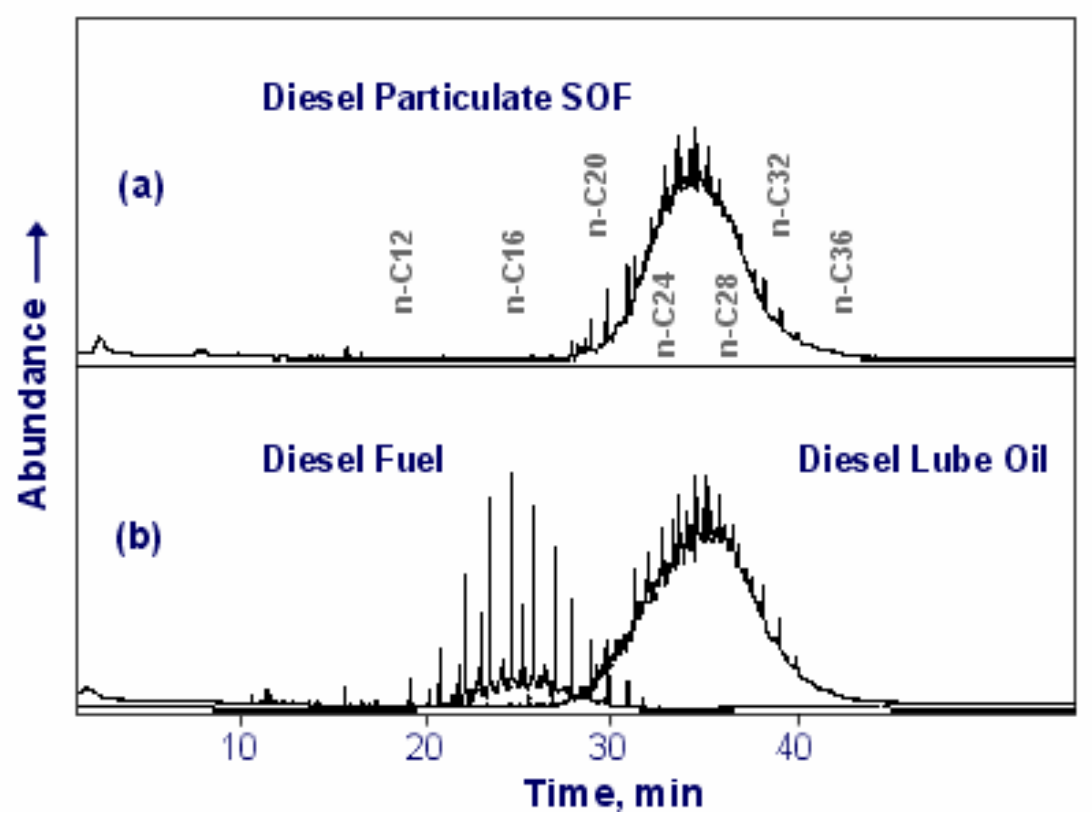

Figure 2- 3 A chromatograph of a SOF sample [8]

\subsection{Health Effects}

The most harmful effect of SOF is that it contains most of the PAH and nitro-PAHs emitted with diesel exhaust emissions [1]. PAHs are carcinogens that are linked to heart diseases and respiratory disease [9]. PAHs are made up of one to seven fused aromatic rings joined in various cluster forms as show in Figure 2- 4.
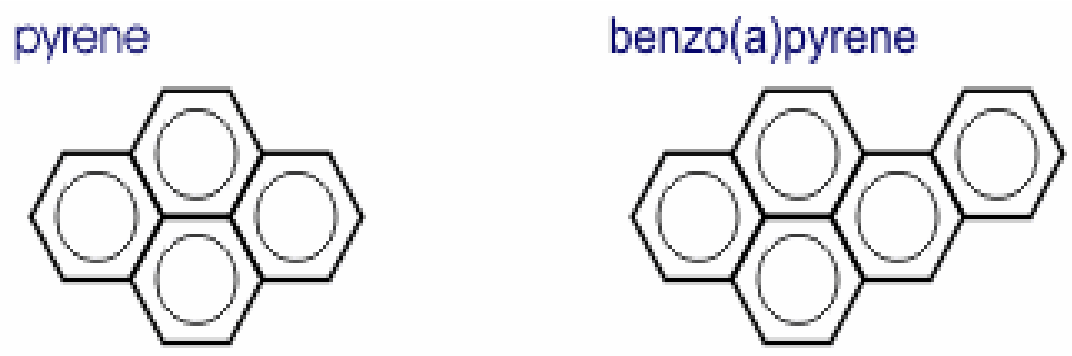

Figure 2- 4 Example of 4 and 5 Ring PAH [8]

PAHs are under close observation by the US EPA, they classify PAHs as an air toxin or polycyclic organic matter (POM). This is a class that is defined by having one or more benzene 
rings and a boiling point of $100^{\circ} \mathrm{C}$ and higher [8]. Seven major POMs are classiefed as a major human carcinogen and include benz(a)anthracene, benzo(b)fluoranthene, benzo(k)fluoranthene, benzo(a)pyrene, chrysene, 7,12-dimethylbenz(a)anthracene, and indeno(1,2,3-cd)pyrene which all are found in SOF.

\subsection{SOF Extraction Techniques}

Since the PM is composed of more than just $\mathrm{SOF}$, such as $\mathrm{SOL}$ and $\mathrm{SO}_{4}$, extraction methods must be used to separate the SOF from all other compounds found in the PM. Three such techniques are microwave, supercritical, and Soxhlet and are detailed below.

\subsubsection{Microwave Extraction}

The use of microwave energy to help in the extraction of organic material was first achieved in the late 1980s [11]. Microwave extraction uses polar organic solvents in contact with solid samples heated in a microwave to extract organic contaminants [12]. Microwave energy is non ionizing radiation; it causes molecular motion by migration of ions and rotation of dipoles. This extraction technique needs a solvent to separate the organic matter from inorganic matter. For normal extraction techniques benzene is used, but benzene is a non-polar solvent, so it is not affected by the microwaves energy. For this reason a polar additive must be introduced such as dichloromethane. When the microwave energy is introduced to the polar solvent, heat is generated and cycled through the PM sample causing the organic material to dissolve.

\subsubsection{Supercritical Extraction}

During supercritical extraction the fluid or solvent is heated and pressurized to its critical point $(\mathrm{CP})$. The critical point is where the vapor and liquid coexist. A fluid reaches a supercritical point (as shown in Figure 2- 5) when the temperature and pressure are higher than 
the corresponding critical values [13]. Once this point is met a fluid can no longer transition to a liquid phase, regardless of the amount of pressure applied.

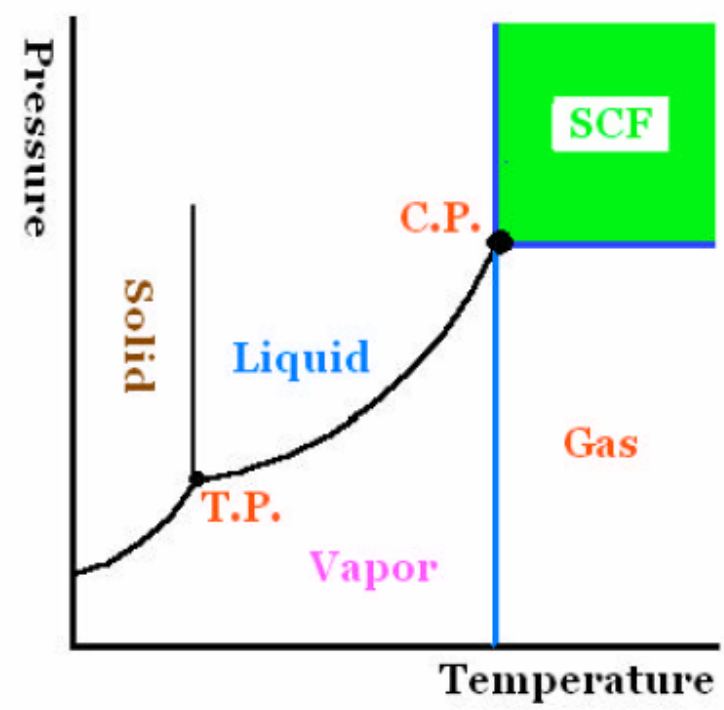

Figure 2- 5 Supercritical point [13]

Fluid at the supercritical point has a much higher diffusivity than for a liquid allowing it to readily penetrate porous and fibrous solids; this can offer excellent catalytic activity [9]. Supercritical fluids can be used to extract SOF from samples. "The main advantages of using supercritical fluids for extractions is that they are inexpensive, extract the SOF faster and more environmentally friendly than organic solvents. For these reasons supercritical fluid $\mathrm{CO}_{2}$ is the reagent widely used as the supercritical solvent [14]."

\subsubsection{Soxhlet Extraction}

Soxhlet extraction was invented in 1879 by Franz von Soxhlet and is illustrated in Figure 2- 6. The Soxhlet process works by placing the PM sample into a thimble (4) made from either thick filter paper or glass with a false bottom made out of a filter substance [15]. The thimble is placed onto a flask containing the solvent (in most cases dichloromethane is used but there are many other solvents depending on what the extraction is for). The solvent is then heated to the 
boiling point which enables the solvent vapor to travel up the distillation arm and condenses in the thimble. The top of the apparatus has a condenser to ensure none of the solvent is able to escape, it cools down and drips back to the stirred bath (2). The chamber housing the thimble then completely fills with warm solvent dissolving part of the organic matter [15]. Once the solvent reaches the level indicated at (6), the solvent is then automatically emptied by the siphon arm (7) taking the dissolved organic material out of the sample. This process is repeated up to 600 times and may take as long as 24 hours to complete and fully dissolve all of the SOF. After the extraction has completed the PM filter sample is permitted to completely dry or evaporate all of the solvent and only leave the inorganic material

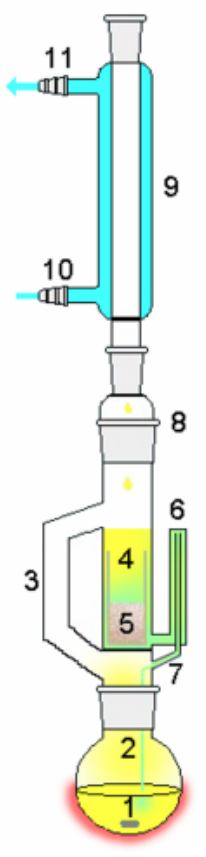
[15].

Figure 2- 6 Soxhlet extractor [8]

\subsection{Extraction Results}

\subsubsection{Microwave Extraction}

One microwave extraction study used different concentration of a dichloromethane and benzene mixture to extract the PM sample. In this study, the dichloromethane-benzene ratio was changed over ten different tests from a molar fraction of 0.1475 to 0.8425 and a run time from 10 to 32 minutes. Since a dual solvent was used two maximum extraction cases are observed as shown in Figure 2- 7 [10]. One instance is high dichloromethane-benzene ratios with low temperatures and high extraction times. The other case of low dichloromethane-benzene ratios with higher temperatures and shorter extraction times [10]. 


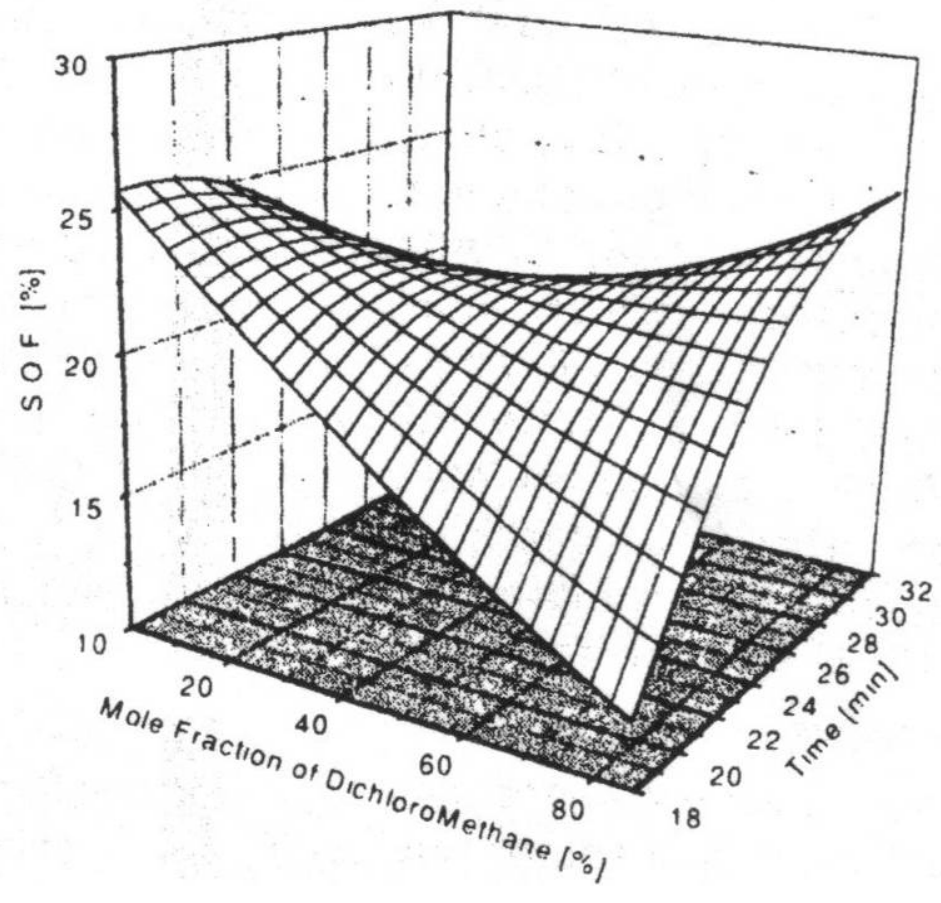

Figure 2- 7 Results of factorial design in microwave extraction of SOF in diesel PM [10]

\subsubsection{Supercritical Extraction}

Supercritical extraction was performed using $\mathrm{CO}_{2}$ and $\mathrm{CH}_{2} \mathrm{Cl}_{2}$ as a modifier. The process was performed at the highest temperature and pressure achievable $\left(\mathrm{T}=130^{\circ} \mathrm{C}\right.$ and $\mathrm{P}=300 \mathrm{~atm}$ due to lab restrictions). Supercritical extraction only needs 40 minutes to complete an entire test and shows similar results as microwave extraction [10].

\subsubsection{Soxhlet Extraction}

For the same PM samples, experiments were performed changing the molar fraction of dichloromethane in benzene from .025 to 1 . Also the number of cycles was increased from 100 to 400 [16]. As shown in Figure 2- 8 the optimal condition for extraction is a dichloromethanebenzene ratio of 70:30 and a relatively high number of cycles. This can be explained because hydrocarbons with low molecular weight are extracted more easily using a solvent that is heavy in dichloromethane, whereas hydrocarbons that are heavier in molecular weight need a solvent 
containing benzene in order to extract the most possible SOF. This is why a binary mixture solvent is beneficial over a single component solvent for the SOF extraction process [10].

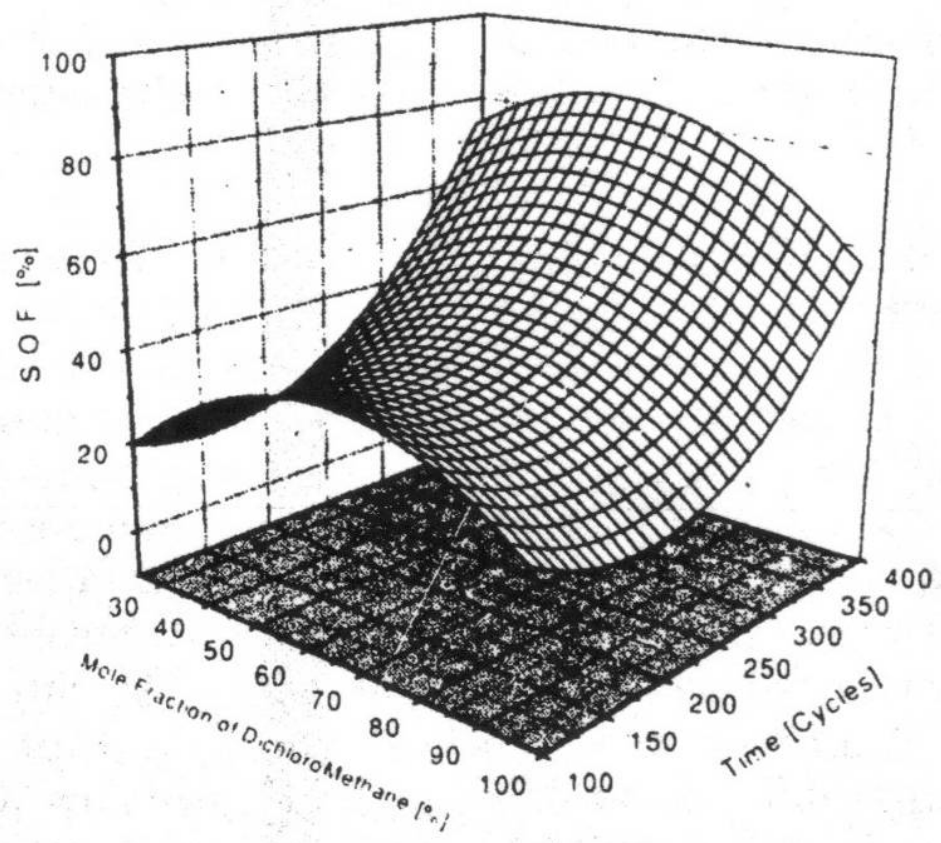

Figure 2- 8 Results of factorial design in Soxhlet extraction of SOF in diesel PM [10] 


\section{Experimental Procedures and Equipment}

\subsection{Introduction}

All experiments conducted for this research were performed at the Center for Alternative Fuels, Engines, and Emissions (CAFEE) at West Virginia University which operate following the 40 CFR Part 86, Subpart N and the standards set by ISO 8178 [2]. There were five different engines used to gather particulate matter for this experiment: 1991 Detroit Diesel Corporation S60, 1992 Detroit Diesel Corporation S60, 1992 rebuilt Detroit Diesel S60, 1999 Cummins ISM 370, and 2004 Cummins ISM 370. This chapter will go into detail to explain all equipment and procedures used to perform this study.

\subsection{Exhaust Gas Analyzers}

Although not directly related to the SOF procedures, the gaseous emissions sampling system is used in the collection of emissions at CAFEE during an emissions test. A description of these systems is provided here to provide a complete description of the laboratory. Additionally, the gaseous emissions data provided a means to verify the repeatability of the engine emissions. The gaseous emissions data are not discussed in this work.

The WVU CAFEE has an array of gas analyzers, heated sampling probes, heated pumps, and heated sampling lines to measure $\mathrm{HC}, \mathrm{NOx}, \mathrm{CO}$, and $\mathrm{CO}_{2}$. These sensors are located in the exhaust emission analyzer bench [Figure 3-1] that receives exhaust emission via the full scale dilution tunnel (explained in a latter section). These analyzers are calibrated in accordance with 40 CFR Part 86, Subpart N using a 10-point calibration curve that is certified within an accuracy of $1 \%$ traceable to National Institute of Standards and Technology (NIST). 


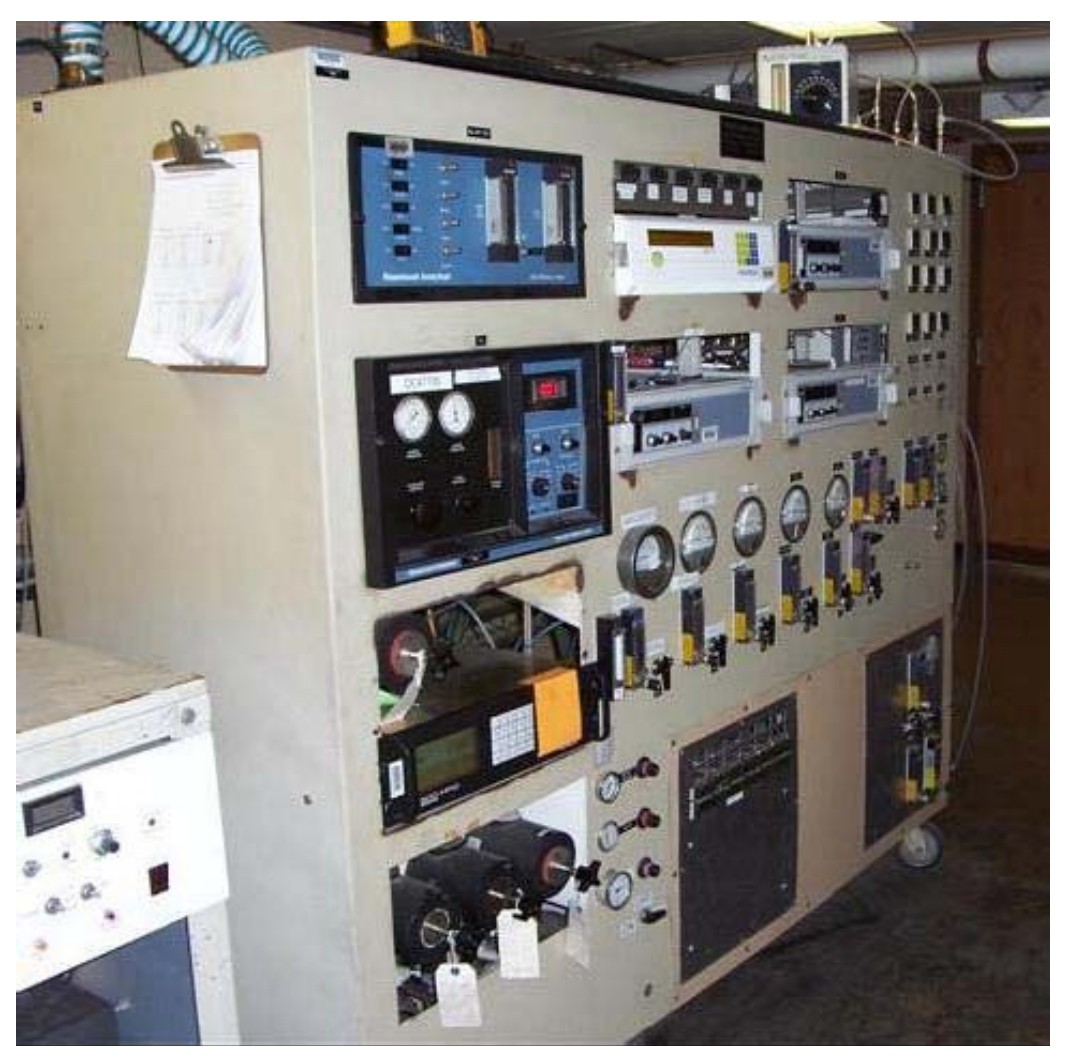

Figure 3- 1 Exhaust Analyzer Bench

\subsubsection{Hydrocarbon Analyzers}

HC is measured using a Rosemont Analytical Model 402 with HFID technology for measuring $\mathrm{HC}$ concentrations. This analyzer uses an internal filter and pump and receives exhaust emissions via a heated line, maintained at $375 \pm 20^{\circ} \mathrm{F}$ to prevent condensation of heavier hydrocarbons. Once passed through the internal filter a flame composed of $60 \%$ helium and $40 \%$ hydrogen burns the hydrogen-carbon molecules. This creates positively charged ions and electrons. A cathode and an anode are used to attract these ions and electrons, which generates current that is proportional to the amount of carbons atoms present in the exhaust gas. This current is processed into an analog voltage with a readable output of 0.0 to 100.0. This represents zero to full scale of the calibration gas used. 


\subsubsection{Oxides of Nitrogen (NOx) Analyzer}

NOx is measured using the Rosemount Analytical Model 955 CLA as a primary device while a secondary device, the Eco Physics CLD $844 \mathrm{CM}$ h, provides for an independent NOx value for quality assurance. Both these analyzer use the principle of chemiluminescence. Any $\mathrm{NO}_{2}$ in the dilute sample stream is converted to $\mathrm{NO}$ by a catalyst inside the analyzer. This $\mathrm{NO}$ stream is then reacted with ozone to produce $\mathrm{NO}_{2}$. In this excited state, a small portion of the molecules decay by emitting photons. Using a photo-detector, the analyzer can measure the amount of light given off by the decaying molecules and convert this light signal into a proportional voltage. An analog voltage is displayed from 0 to $5 \mathrm{~V}$ that represents zero to full scale of the calibration gas used.

\subsection{2 $\mathrm{CO}_{2}$ and $\mathrm{CO}$ Analyzers}

$\mathrm{CO}_{2}$ is measured using the HORIBA AIA-210 NDIR whereas CO uses the HORIBA AIA-210LE NDIR. A NDIR (non-dispersive infrared) analyzer for $\mathrm{CO}_{2}$ and $\mathrm{CO}$ works by emitting only light with the wavelength that the gas being measured can be absorbed. The gas concentration is then measured electro optically by its absorption of the specific wavelength and then converted to a full scale read out. In the CAFEE laboratory, two CO analyzers are used, a low-range analyzer to measure $\mathrm{CO}$ up to $1000 \mathrm{ppm}$ and a high analyzer to measure $1000 \mathrm{ppm}$ to $5000 \mathrm{ppm}$. This allows a more accurate measurement of the full span of CO emissions based on the type of engine and test cycle being run.

\subsection{Full Flow Exhaust Dilution Tunnel}

To simulate real world environments when analyzing diesel exhaust emissions, a full scale dilution tunnel is implemented. This tunnel provides ambient air to mix with raw diesel exhaust emissions in order for reactions to occur to simulate exhaust entering the atmosphere. This also allows exhaust gases to cool and eliminates any condensates that may occur. The 
dilution tunnel is composed of an 18inch diameter stainless steel duct, 40feet long as shown in Figure 3- 2.

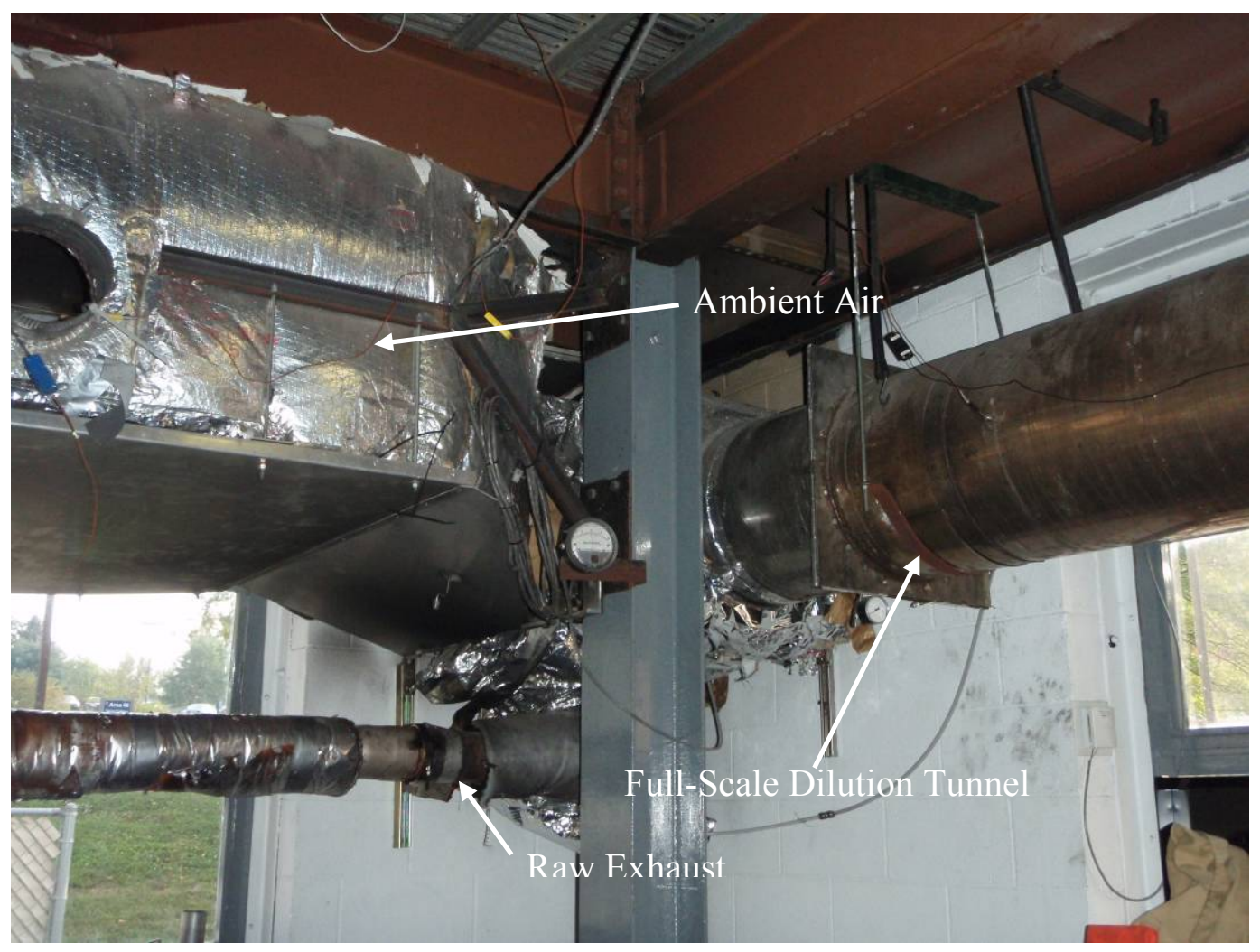

Figure 3- 2 Full Scale Dilution Tunnel

Temperature controlled ambient air is pulled through up to four critical flow venturis (CFV) using a 75hp blower. At the entrance to the tunnel, the raw exhaust and ambient air introduced at a mixing orifice to promote adequate mixing of these two streams. The four CFVs are composed of one $400 \mathrm{scfm}$ venturi and three $1000 \mathrm{scfm}$ venturis. These venturis can be opened or closed to provide a total flow rate of $3400 \mathrm{scfm}$. The venturi flow rate is proportional to temperature and pressure thus allowing the mass flow rate through the tunnel to be calculated using the equation below:

$$
Q(s c f m)=K_{v} \frac{P_{a b s}}{\sqrt{T_{a b s}}} .
$$

Equation 3- 1 
Where, $\mathrm{Q}(\mathrm{scfm})$ is the standard volumetric flow rate at $29.92 \mathrm{inHg}$ and $68^{\circ} \mathrm{F}, \mathrm{K}_{\mathrm{v}}$ is the calibration constant for the operating venturi, $\mathrm{P}_{\mathrm{abs}}$ is the absolute pressure before entering the venturi, and $\mathrm{T}_{\mathrm{abs}}$ is the absolute temperature of the diluted exhaust before the venturi entrance.

The sampling probes for the analyzer are placed 10 diameters (180inches) downstream to collect the diluted exhaust as shown in Figure 3- 3. The diluted exhaust is then piped to the sensors using heated lines and filters to prevent condensation.

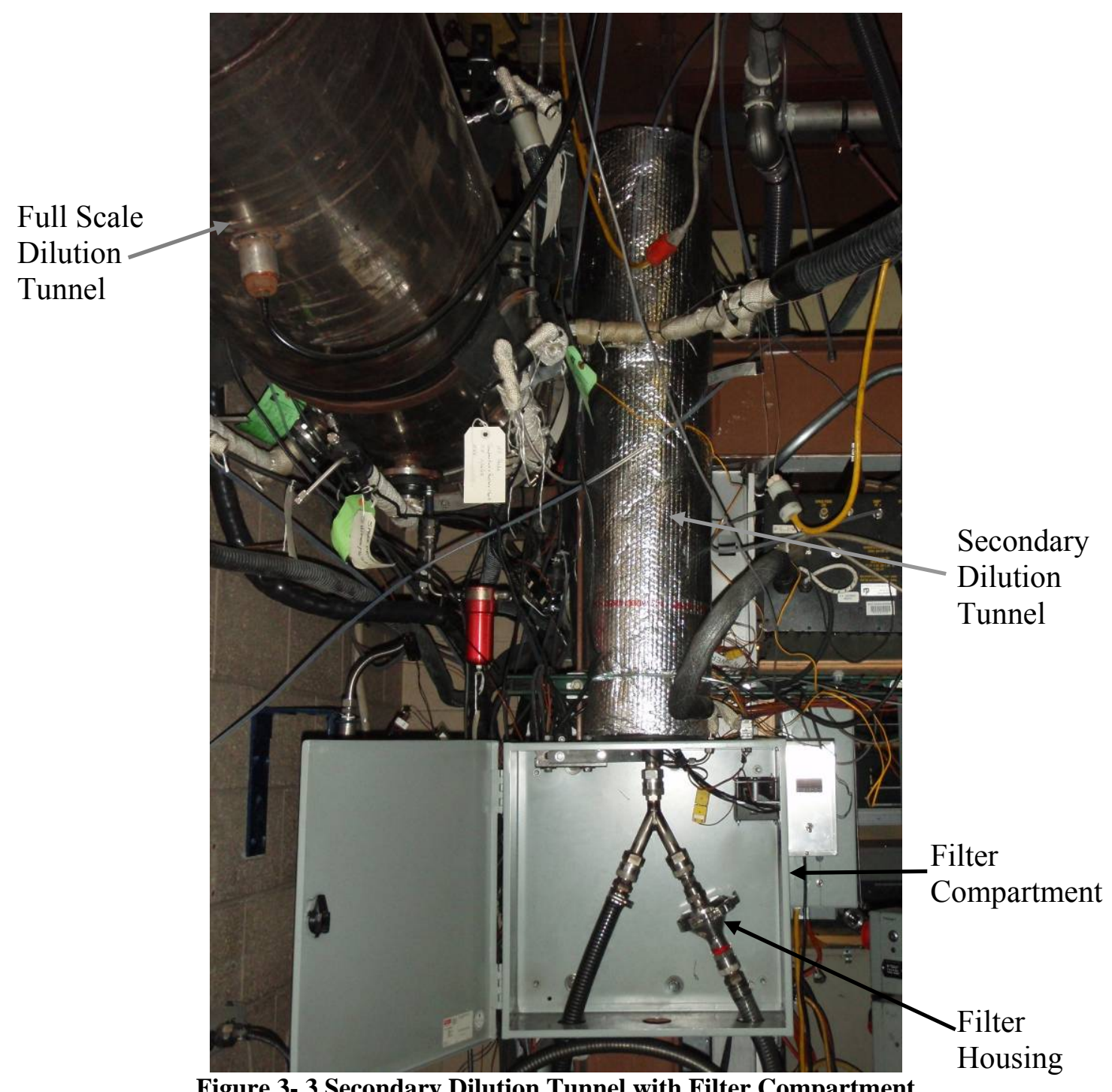

Figure 3- 3 Secondary Dilution Tunnel with Filter Compartment 


\subsection{Particulate Matter Sampling System.}

Particulate matter is gathered using a gravimetric-based approach. A slipstream is taken from the main dilution tunnel and routed to a secondary dilution tunnel. This secondary dilution tunnel, as shown in Figure 3- 3 and Figure 3-4, is held to a proportionally equivalent mass flow rate of the main dilution tunnel.

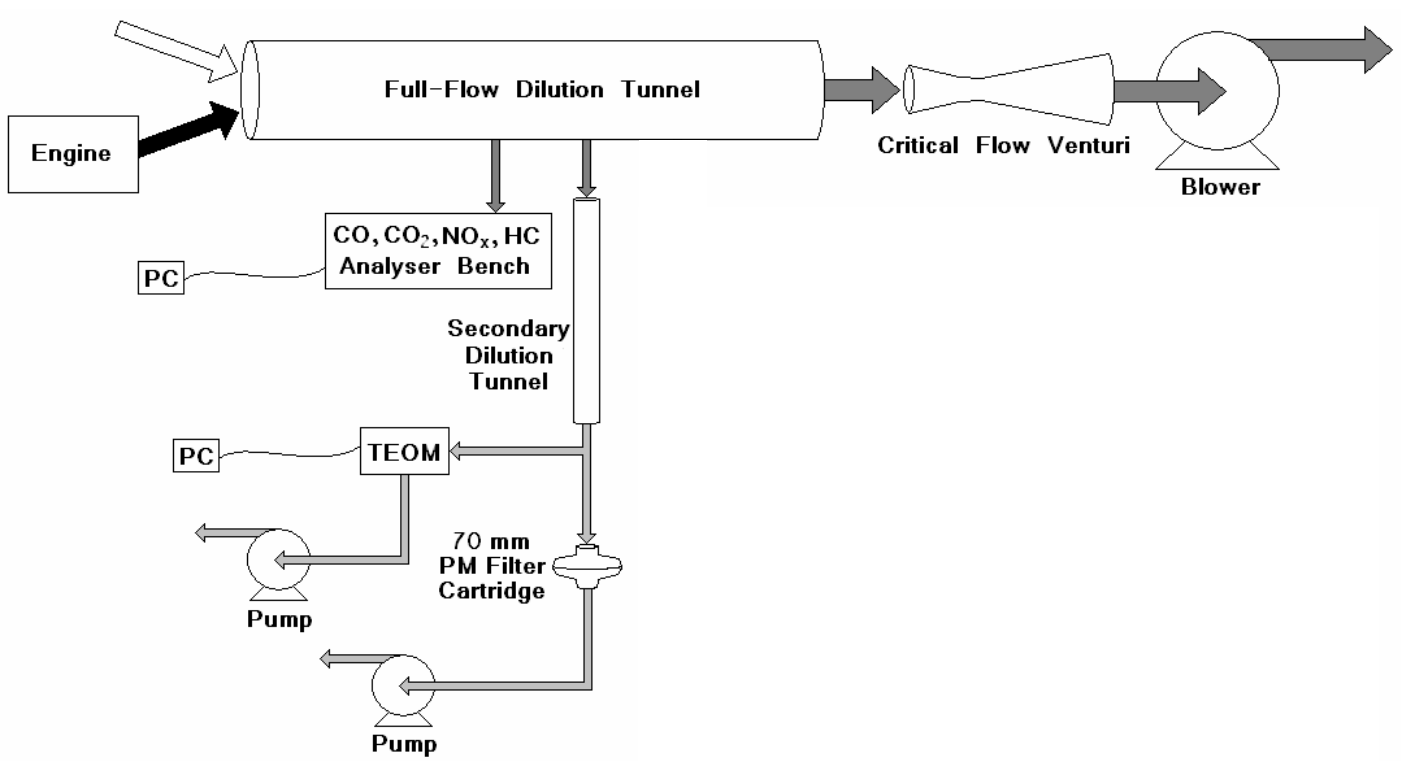

Figure 3- 4 Schematic of Full Scale and Secondary Dilution Tunnels

The diluted air is drawn through a stainless steel filter housing (shown in Figure 3- 3,

Figure 3- 5, and Figure 3- 6), containing a primary and secondary T60A20 70mm Pallflex fluorocarbon coated glass filter. The volumetric flow rate through this secondary dilution tunnel is regulated using a mass flow controller. The mass flow rate is calibrated yearly using a laminar flow element (LFE) manufactured by Meriam Instruments (Model No. 50MC2-6) as specified by the CAFEE Emissions Laboratory Standard Procedures [18]. The filter face is maintained at a temperature below $52^{\circ} \mathrm{C}$ per requirements of the 40 CFR Part 86, Subpart N [2]. 


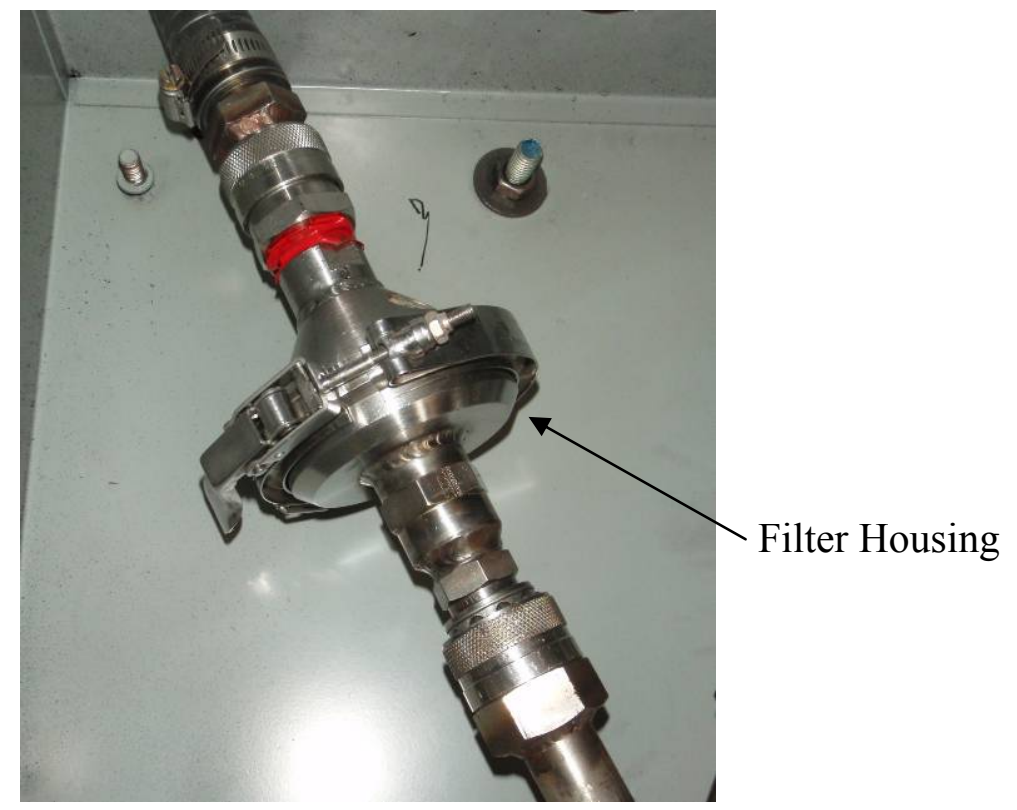

Figure 3- 5 Stainless steel filter holder connected in line with the Secondary Dilution Tunnel

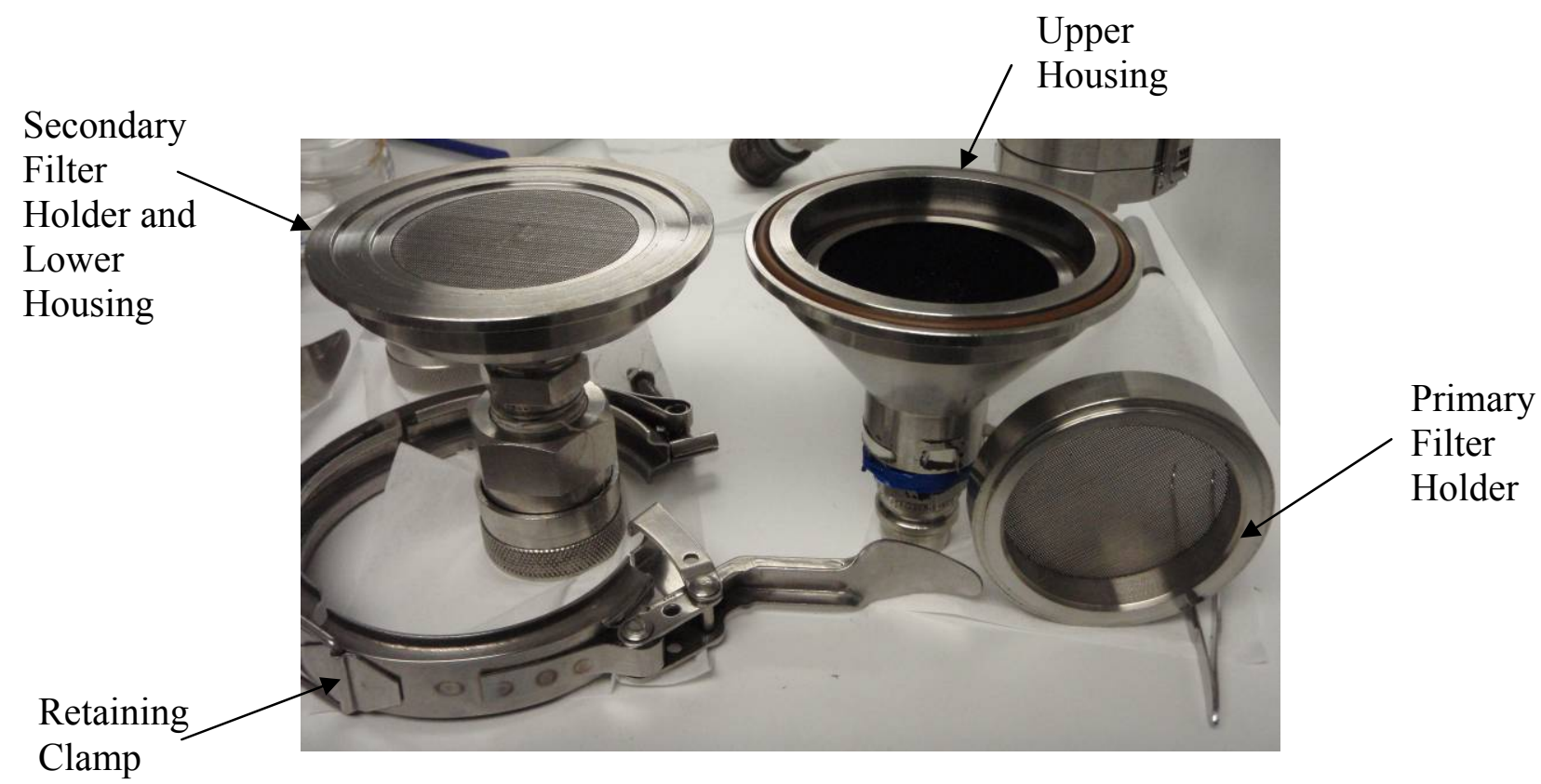

Figure 3- 6 Stainless steel filter housing separated into it's components

\subsection{Clean Room Weighing and Filter Conditioning}

The WVU CAFEE has an ISO Class 6 clean room, where all conditioning and weighing of filters are performed. Although this clean room exceeds the requirements for filter weighing for the engines tested, it was used in this program. As specified in 40 CFR Part 86, Subpart N 
[2], the filters are pre-conditioned in an environmentally controlled room for at least one hour before PM weighing. The room is maintained at a temperature of $22 \pm 3^{\circ} \mathrm{C}$ and a dew point of $9.5 \pm 1{ }^{\circ} \mathrm{C}$. Initially, the filters are pre-conditioned before the initial pre-weight. Prior to the initial pre-weight, reference filters are weighed during each weighing session to track the amount of variation on these filters as a quality check. These reference filters are maintained for the entire length of the emissions testing period. After the reference filters are weighed, the test filters are weighed to obtain the filter's pre-weight before the test. The filters are weighed using a Sartorius SE2-F ultra-microbalance. This microbalance has a readability of $0.1 \mu \mathrm{g}$ and a repeatability of $\pm 0.25 \mu \mathrm{g}$ [19]. Once a test cycle has been completed and PM is collected, the filters are placed back into the clean room and brought back to equilibrium condition for at least one hour before post weighing. The microbalance located in the clean room is shown in Figure 3- 7. The post test weight is collected in a similar manner as the pre-weight measurement.

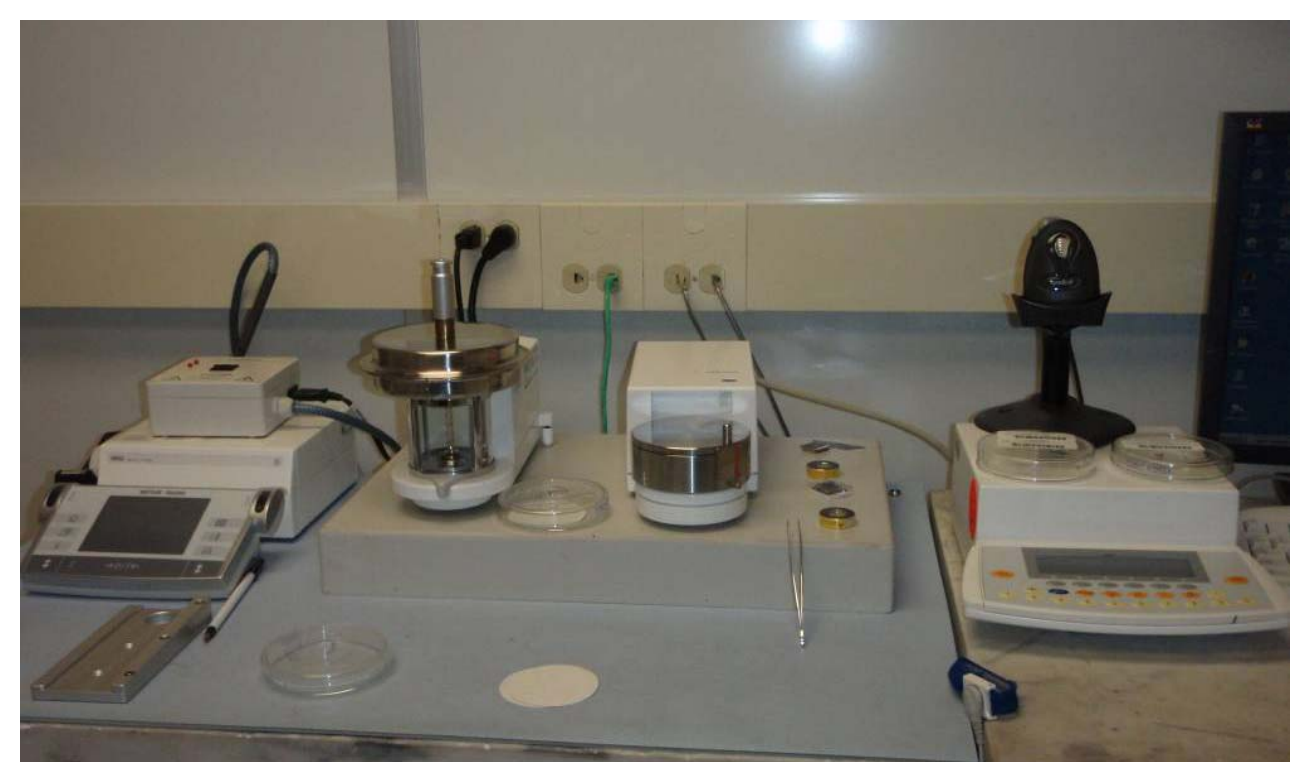

Figure 3- 7 Microbalance

An in-house computer software system was designed and written to record all weight measurements gathered from the reference filters and PM deposited filters. This software utilizes 
a barcode scanning system, which enables the user to track all filters by scanning a unique barcode that is assigned to each Petri dish housing the filters. The software then tracks each weight entered, time, date, and user. The software also tracks temperature, humidity, dew point and records a running average to alert the user if the clean room measure was out of allowed tolerance in any of these areas. Finally this system is used to record when a filter has been checkout of the clean room for testing, when the filter has been returned to the clean room, and when the filter has been in equilibrium conditions for at least one hour. If the filter is then tried to be post weighed and has not meet the one hour requirement, it will alert the user and not allow the weight measurement to be recorded. A visual display is shown in Figure 3- 8 of the software system.

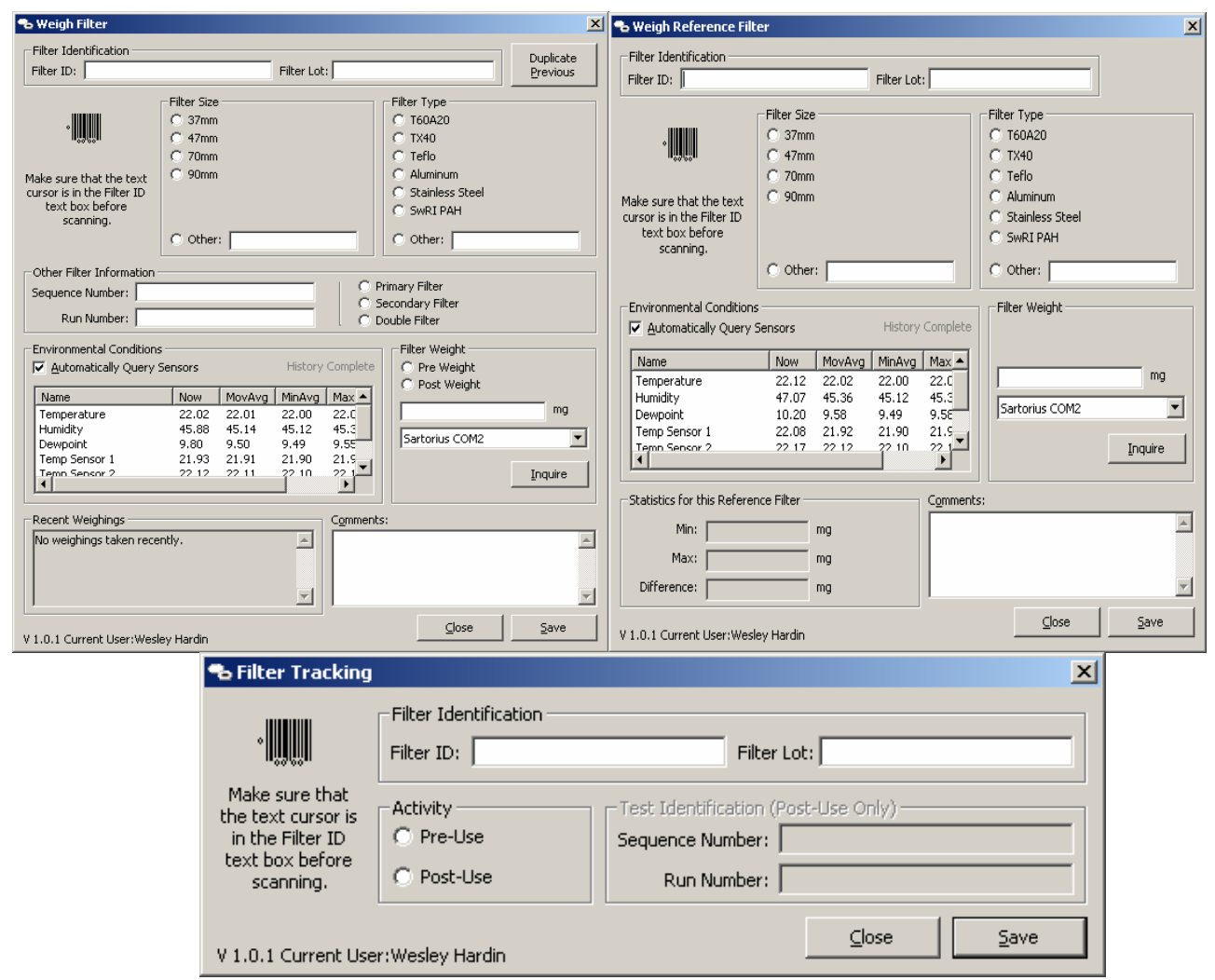

Figure 3- 8 Filter weight tracking software 


\subsection{SOXHLET Extraction}

\subsubsection{Introduction}

Soxhlet extraction is a process that involves placing a filter with deposited PM into an extraction device to dissolve all organic matter in the PM while leaving only inorganic matter behind. From this, the soluble organic fraction can be calculated. The Soxhlet process used for this study is governed by the "Test Method for Soluble Organic Fraction (SOF) Extraction" [20] as stated by the State of California Air Resources Board. Although there are other processes and solvents that may be used for SOF determination, the California Air Resources Board method was of interest since the CAFEE laboratory performs research on behalf of the California Air Resources Board and prior test programs using external laboratories to extract the SOF has shown high variability in the SOF data. The following section will explain in detail how CAFEE uses the Soxhlet extraction process to measure the SOF following the California Air Resources Board requirements.

\subsubsection{Soxhlet Laboratory Setup}

The Soxhlet extraction process uses six Ace Glass 6716 extraction apparatus, a Glas-Col heating mantle, and Glas-Col heating control module as shown in Figure 3- 9. The extraction apparatus is made up of four parts: (from top to bottom) condenser, $45 / 50$ extractor, thimble, and $125 \mathrm{ml}$ flask. The condenser has cold tap water pipe to it, entering at the bottom and exiting at the top. Each condenser is linked to the next unit to providing cooling for the vaporized solvent to condense and drain back into the extractor. The whole assembly is contained inside a chemical hood to prevent fumes escaping to the room. 


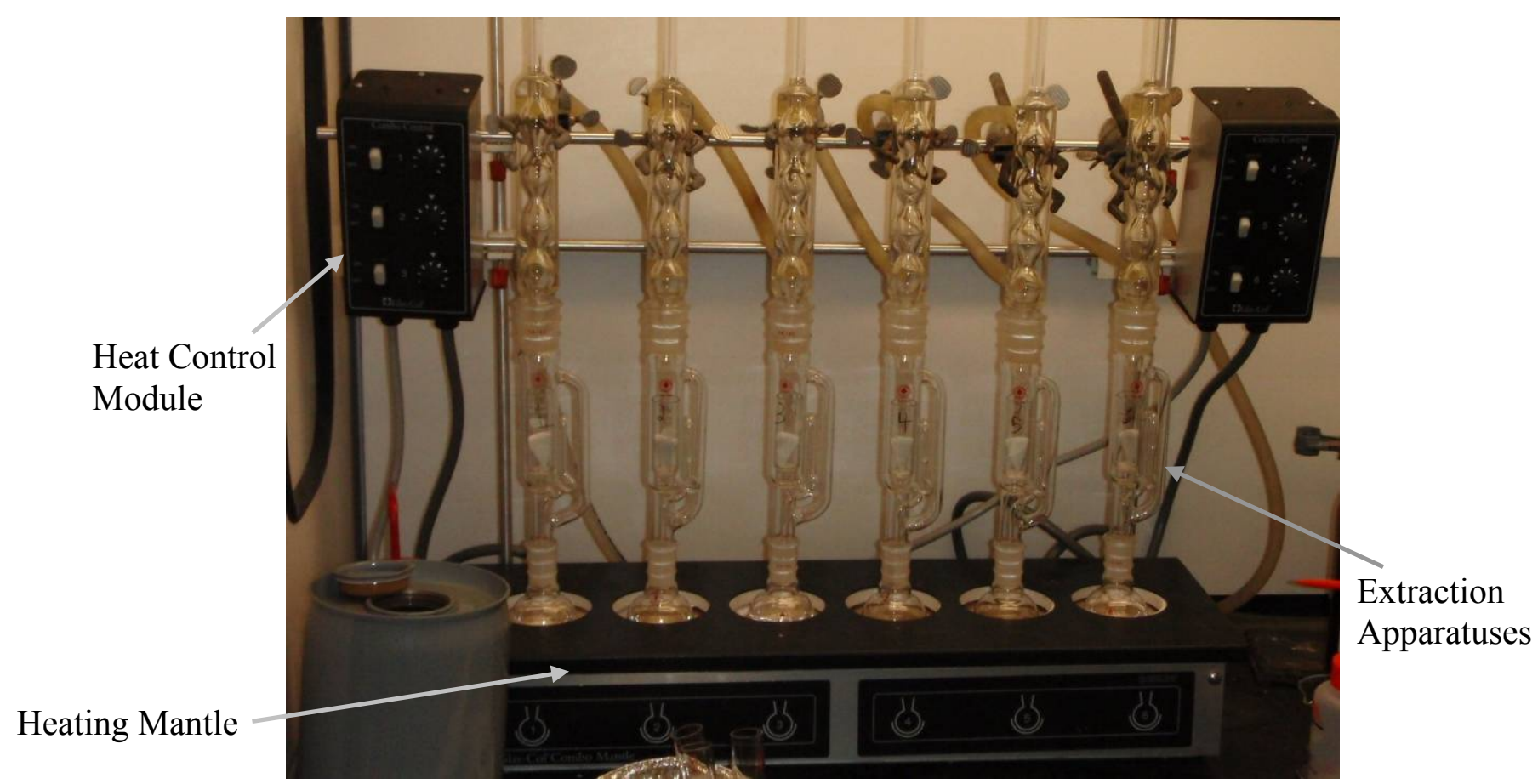

Figure 3- 9 Extraction Apparatus, Heating Mantle, and Heat Control Module

\subsubsection{SOXHLET Extraction Procedure}

To use the extraction apparatus, first the filter pair with the deposited PM must be folded using forceps and a spatula. This is done by laying the primary and secondary filter with PM sided towards one another on a clean stainless steel work surface. The two filters are then folded in halves, then quarter, and finally eights while only being touched with the forceps and spatula, as shown in Figure 3-10. This is done in the clean room to prevent containments from depositing on the filters. The filter is then placed into a glass thimble as shown in Figure 3-11. The thimble and filter are then placed into a sealed container for transportation to the extraction apparatus located in the Engineering Sciences Building. Once at the extraction apparatus, $80 \mathrm{ml}$ of solvent is placed into the flask along with three boiling chips (Boileezers). The solvent used for this process was composed of a toluene:ethanol binary solvent 32:68 wt/wt. with a boiling point of $76.7^{\circ} \mathrm{C}$ as required by the California Air Resources Board test method [20]. Boiling chips are used to prevent the solvent from becoming superheated and boiling violently [21]. The thimble is 
removed form the container, placed carefully into the extractor, and the whole assembly is then placed onto the heating mantle. Note that rubber gloves are used to handle the thimble and glassware to prevent contamination of the glassware with oils from the skin and to prevent the solvent contacting the skin. The heat control module and the water valve are turned on. The heat control module is set to apply heat to the flask such that the solvent is cycled though the extraction process once every 15 minutes. Once the solvent has cycled for 8-12 hours (32 to 48 cycles), the heat is turned off and the extractor is allowed to cool. The apparatus is then taken apart and the thimble is removed from the extractor. The thimble with the filter is left in the chemical hood for six hours to allow the filter to dry. After the filter has dried, it is placed back in the sealed container and transported to the clean room. Therein the clean room, the filter is removed from the thimble using forceps, placed into a Petri dish, and allowed to reach equilibrium for at least one hour. The filter is then weighed, the weight recorded, and the SOF determined. 


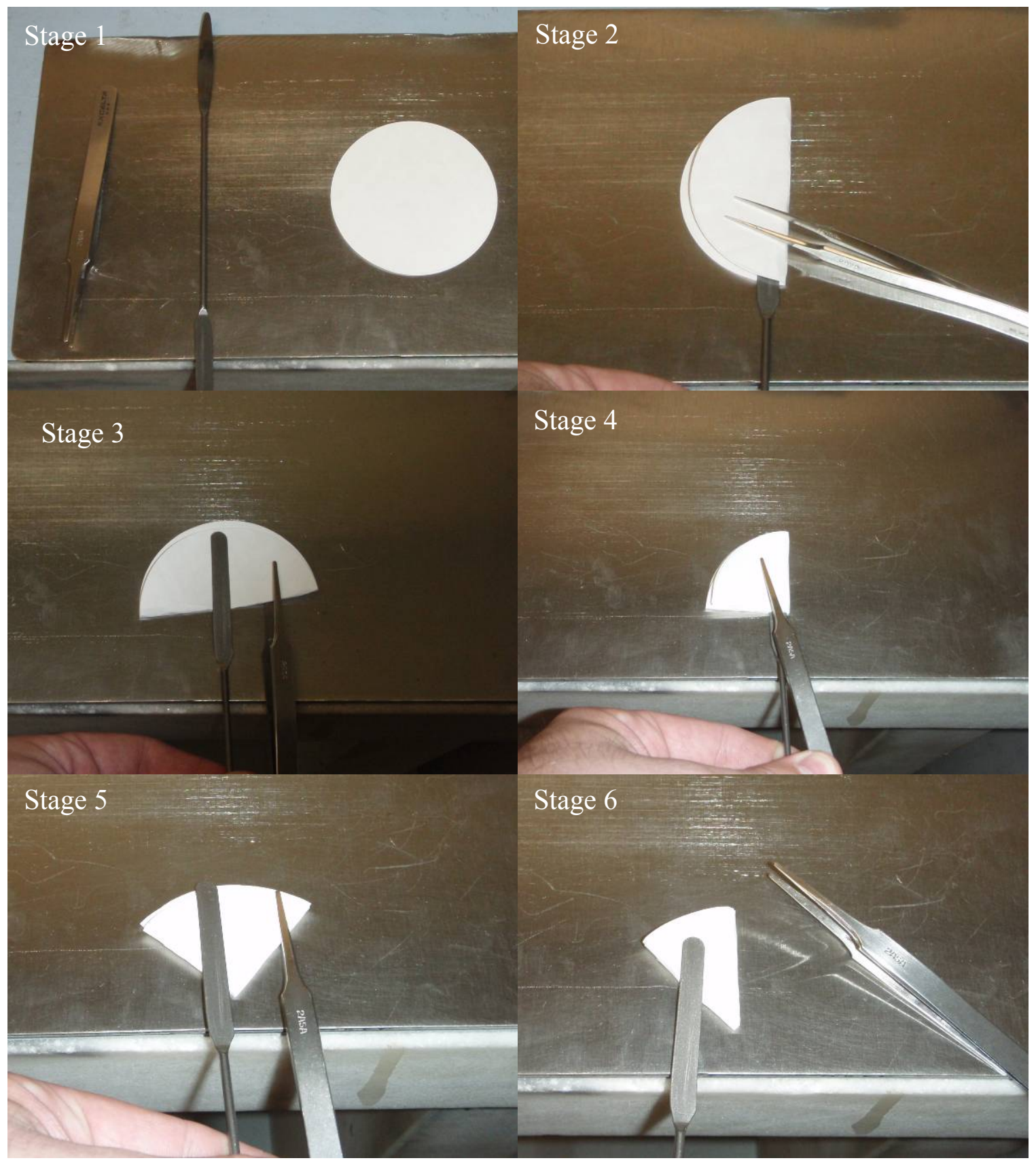

Figure 3- 10 Filter Folding Procedure 


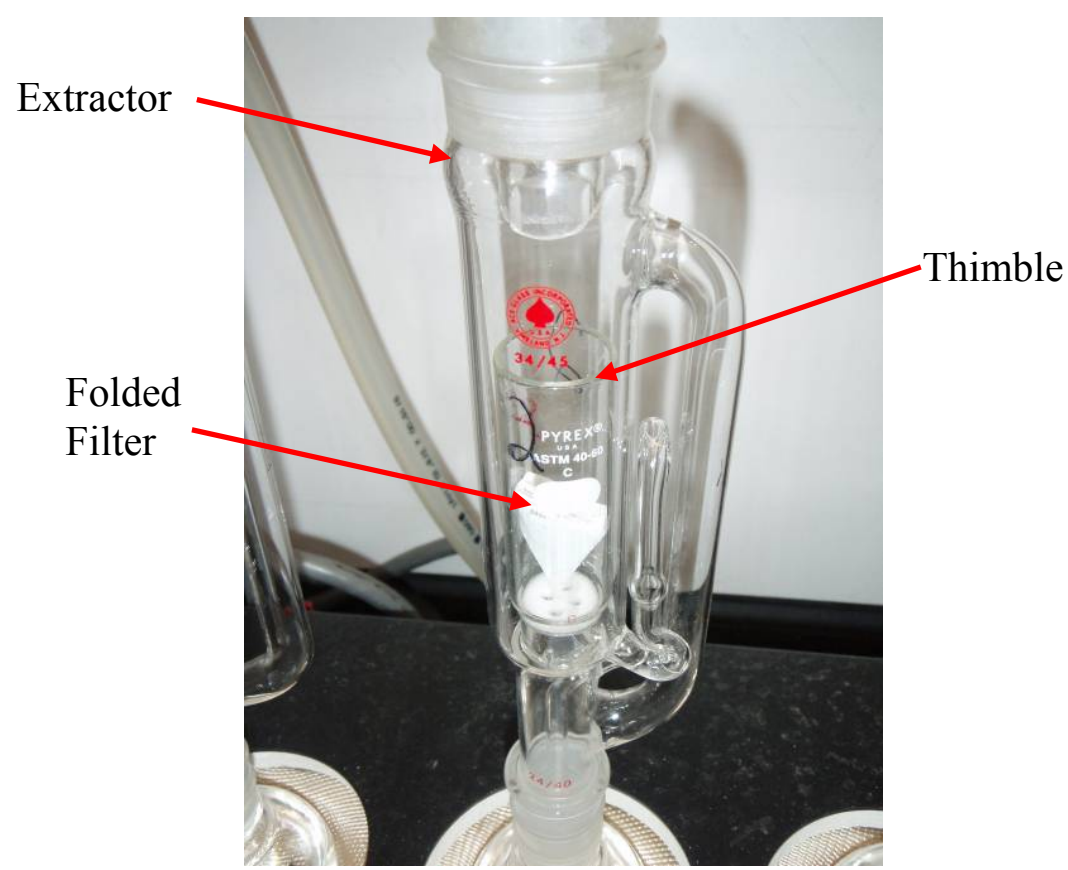

Figure 3- 11 Thimble with filter inserted into extractor

\section{Experimental Configuration}

The PM-loaded filters used for this work were obtained from prior studies where the filters were stored in their original glass Petri dishes or in anti static bags. The prior studies were generally from a 1992 Detroit Diesel Corporation Series $60 \mathrm{HDDE}$ with the same fuel but at different time periods. Although different filters were used from different time periods with possible different fuels, the experiments conducted in this study were selected to use the same batch of filters from a common program to examine test-to-test variability. In other words, the uncertainty in the SOF measurement from a batch of filters were of interest and not the absolute value. It is noted that 40 CFR Part 86 , Subpart N requires reference filters to be changed out during monthly intervals and that test filters' pre and post weights be done with the same reference filter pair. Due to the nature of the experiments being conducted over many months, this requirement was not meet. However, additional reference filters were collected to account for the extended duration of this program. It is also noted that the reference filters used for the 
PM-loaded filters net mass determination and the reference filters used for the reporting of this data were generally different.

Only $70 \mathrm{~mm}$ T60A20 fiber filters were used in this study since these filters are specifically called for in 40 CFR Part 86 , Subpart $N$ for the testing required by the California Air Resources Board. Two filters were used as a pair with the nominal mass of these filters pair of 300mg. The net mass from an FTP test was on the order of four to five mg. The uncertainty (one standard deviation) in repeated reference filter weighing was approximately $0.006 \mathrm{mg}$ (6 micrograms), the uncertainty in the zero reading of the scale was approximately $0.001 \mathrm{mg}$ (1 microgram), and the uncertainty in the calibration reading of the scale was approximately $0.0007 \mathrm{mg}(0.7$ microgram $)$. An overall uncertainty in the measurement of a filter is assumed to be $0.010 \mathrm{mg}$ (10 micrograms) as required by 40 CFR Part 86 , Subpart N. The variations listed were from a span of more than a one month period and from different personnel weighing the filters.

For this research, the CARB regulations govern that the test must be a minimum of 40 cycles as a guideline. Each cycle takes a minimum of 12 minutes to complete, for a total cycling time of 8 hours. It is noted that cycling time could last up to 72 hours for different applications for Soxhlet extraction such as if organic matter being further extracted and tested for the individual species; however, the cycle times in this program were less than 24 hours. 
Table 3- 1 Test Performed and Description

\begin{tabular}{|c|c|c|c|}
\hline \multicolumn{4}{|c|}{ Tests Performed } \\
\hline \multicolumn{2}{|r|}{ SOF Variability } & \multicolumn{2}{|r|}{ Shipping and Weighing } \\
\hline & & Type of Test & $\begin{array}{c}\text { Description } \\
\end{array}$ \\
\hline Type of Test & Description & \begin{tabular}{|l|} 
Set 1 \\
Control
\end{tabular} & $\begin{array}{c}\text { Clean filters placed in clean room and weighed } \\
\text { continuously for a month }\end{array}$ \\
\hline COV\% of SOF & $\begin{array}{l}57 \text { sets of PM Deposited filters } \\
\text { extracted and weighed }\end{array}$ & $\begin{array}{l}\text { Set } 2 \\
\text { Petri Ship } \\
\text { Control }\end{array}$ & $\begin{array}{c}\text { Clean filters } \\
\text { placed in Petri dishes then shipped, returned, and } \\
\text { weighed }\end{array}$ \\
\hline \multicolumn{2}{|r|}{ Weight Gain over Time } & $\begin{array}{l}\text { Set } 3 \\
\text { Petri Ship } \\
\text { Extract }\end{array}$ & $\begin{array}{l}\text { PM deposited filters } \\
\text { shipped, returned, extracted, and weighed }\end{array}$ \\
\hline Type of Test & Description & \begin{tabular}{|l} 
Set 4 \\
Anti-Static \\
Ship Control \\
\end{tabular} & $\begin{array}{l}\text { Clean filters placed in anti-static bags, shipped, } \\
\text { returned, and weighed }\end{array}$ \\
\hline $\begin{array}{l}\text { Set } 1 \\
\text { Control }\end{array}$ & $\begin{array}{c}\text { Clean filters placed in clean room and } \\
\text { weighed } \\
\text { continuously for a month }\end{array}$ & $\begin{array}{l}\text { Set } 5 \\
\text { Anti-Static } \\
\text { Extract }\end{array}$ & $\begin{array}{l}\text { PM deposited filters placed in anti-static bags, } \\
\text { shipped, returned, extracted, weighed }\end{array}$ \\
\hline $\begin{array}{l}\text { Set } 2 \\
\text { Transport } \\
\text { Control } \\
\end{array}$ & $\begin{array}{l}\text { Folded, place in SOF thimble transported } \\
\text { to experiment room and back to clean room }\end{array}$ & $\begin{array}{l}\text { Set } 6 \\
\text { Freezer Petri } \\
\text { Control }\end{array}$ & $\begin{array}{l}\text { Clean Filters, placed in petri dishes, } \\
\text { placed in freezer, placed in clean room, weighed }\end{array}$ \\
\hline $\begin{array}{l}\text { Set } 4 \\
\text { Soxhlet } \\
\text { Extraction }\end{array}$ & $\begin{array}{l}\text { Soxhlet Extraction performed on filters with } \\
\text { PM and continuously weighed for a month }\end{array}$ & \begin{tabular}{|l} 
Set 7 \\
Freezer Petri \\
Extract \\
\end{tabular} & $\begin{array}{l}\text { PM deposited filters, placed in petri dishes, } \\
\text { placed in freezer, extracted, weighed }\end{array}$ \\
\hline $\begin{array}{l}\text { Set } 5 \\
\text { Sonication }\end{array}$ & $\begin{array}{l}\text { Clean filters, first sonicated, then extracted, } \\
\text { then weighed continuously for a month }\end{array}$ & $\begin{array}{l}\text { Set } 8 \\
\text { Freezer Petri } \\
\text { Ship Control } \\
\end{array}$ & $\begin{array}{c}\text { Clean Filters, placed in petri dishes, } \\
\text { placed in freezer, shipped, returned, placed in clean } \\
\text { room, weighed }\end{array}$ \\
\hline $\begin{array}{l}\text { Set } 6 \\
\text { Oil deposited }\end{array}$ & $\begin{array}{c}1 \mathrm{mg} \text { of } 15-\mathrm{W} 40 \text { oil deposited on clean filter. } \\
\text { Placed in clean room and weighed } \\
\text { continuously for a month }\end{array}$ & $\begin{array}{l}\text { Set } 9 \\
\text { Freezer Petri } \\
\text { Ship Extract }\end{array}$ & $\begin{array}{l}\text { PM deposited filters, placed in petri dishes, } \\
\text { placed in freezer, shipped, returned, extracted' } \\
\text { placed in clean room, weighed }\end{array}$ \\
\hline $\begin{array}{l}\text { Set } 7 \\
\text { Oven Baking }\end{array}$ & $\begin{array}{l}\text { Clean filters baked in oven at } 375 \mathrm{~F} \text {, } \\
\text { for } 1 \text { hour, then weighed continuously for } \\
\text { one month }\end{array}$ & $\begin{array}{l}\text { Set } 10 \\
\text { Extracted }\end{array}$ & $\begin{array}{l}\text { PM deposited filters, extracted, } \\
\text { placed in clean room, weighed }\end{array}$ \\
\hline
\end{tabular}

The above table is an outline of all test performed for this study. The first set of test

performed were 52 sets of PM deposited filters that were extracted using the Soxhlet extraction

process. These sets of filters were used to test and prove the variability of the test procedure for

determining SOF. The next sets of test performed were to examine the weight gain over time

from different processes that T60A20 filters may experience. Configuration other than just PM

extracted filters were used to try to determine the root cause of post SOF extraction weight gain

in a controlled environment. Finally the last sets of test were performed to test the impact of

shipping had on extraction results. 


\section{Results and Discussions}

\subsection{Introduction}

For this study, three outcomes were desired; first develop a test procedure that would reduce variability for SOF determination when using Soxhlet extraction. The second was to examine how post extraction weight can vary over time in a controlled environment. The last objective was to explore handling issues with the transportation of the filter media. This chapter will explain in detail results for each objective.

\subsection{Variability in SOF Determination}

The main purpose of this study was to deliver a procedure to determine SOF while achieving a low variability of results. This was achieved by creating a test procedure that could be followed by different laboratory personnel while obtaining the same results. While doing this, many factors in SOF determination were observed in order to produce lower variability. In order to test the variability for this procedure, groups of filters were selected that were run as a set of FTP's. Since these filter groups were collected from repeat FTP tests using the same engine and setup (engine set point file, fuel, intake and exhaust settings, etc.) they produced relatively the same amount of deposited PM. Each of these sets were then extracted and weighed at the same time to check variability of the procedure. SOF was found using the following equation.

$$
S O F=\frac{\text { TestMass }_{\text {final }}-\text { ExtractionMass }}{\text { TestMass }_{\text {final }}-\text { TestMass }_{\text {initial }}} \text { Equation 4- } 1
$$


Where the TestMass initial is the mass of the filter set prior to the engine emissions test (pre-test mass), TestMass final is the mass of the filter set after the engine emissions test (post-test mass), and ExtractionMass is the mass of the filter set after the SOF.

Once the SOF is found for individual FTP tests, the coefficient of variation (COV) was calculated using

$$
\text { COV }=\frac{\text { Standard Deviation }_{\text {FTP }}}{\text { Average }_{\mathrm{FTP}}} \times 100 \% \text { Equation 4- } 2
$$

Where the Standard Deviation ${ }_{\text {FTP }}$ is the standard deviation of the set of FTP tests and the Average $_{\text {FTP }}$ is the average of the set of FTP tests. Quantifying the variability is desired because CARB has implemented a passage criterion for all fuel additives being tested as shown in

$$
\overline{\mathrm{x}}_{\mathrm{C}} \leq \overline{\mathrm{x}}_{\mathrm{R}}+\delta-\mathrm{S}_{\mathrm{p}}(\sqrt{2 / \mathrm{n}}) \mathrm{t}(\mathrm{a}, 2 \mathrm{n}-2) . \text { Equation 4- 3 [3] }
$$

Where $\quad \bar{x}_{C} \quad=$ Average SOF during testing with the candidate fuel;

$\bar{x}_{R} \quad=$ Average SOF during testing with reference fuel;

$\delta \quad=$ Tolerance level, equal to 6 percent for SOF;

$S_{p} \quad=$ Pooled standard deviation;

$t(a, 2 n-2)=$ The one-sided upper point of $t$ distribution with $\mathrm{a}=0.15$ and $2 \mathrm{n}-2$ degrees of freedom;

$\mathrm{n} \quad=$ Number of paired candidate and reference fuel tests, generally either 20 or 21 .

As the COV of the SOF extractions is lowered, the pooled standard deviation will lower. The lower the pooled deviation, the larger the $\bar{x}_{C}$ of the candidate fuel can be, without failing the passage criterion set forth by CARB. Equation $4-4$ can be rearranged as: 


$$
\frac{\bar{x}_{C}-\bar{x}_{R}}{\bar{x}_{R}} \leq 0.06-\frac{S_{p}}{\bar{x}_{R}} C \text {. Equation 4- 4 }
$$

Where $\delta$ has been set to $0.06(6 \%)$ and

$$
\mathrm{C}=(\sqrt{2 / \mathrm{n}}) \mathrm{t}(\mathrm{a}, 2 \mathrm{n}-2) . \text { Equation 4- } 5
$$

For 20 tests $(n=20)$ and $\mathrm{a}=0.15, \mathrm{C}=0.33228$ and for 21 tests, $\mathrm{C}=0.32405$. Figure 4 - 1 illustrates the range of allowable variation in the pooled deviation and difference between the candidate and reference fuel SOF values relative to the reference fuel SOF average value. For no variation $(\mathrm{Sp}=0)$ in the test-to-test SOF values, the candidate fuel SOF average value may be $6 \%$ higher than the reference fuel SOF average value. However, as the variation in the test-to-test SOF values increase, the allowable difference between the candidate fuel and reference fuel SOF average values decrease to where the candidate fuel SOF value must be less than the reference fuel SOF value for a pooled variation of $\sim 18 \%$ and the candidate fuel SOF average value must be $10 \%$ lower than the reference fuel SOF average value for a pooled variation of $\sim 48 \%$. 


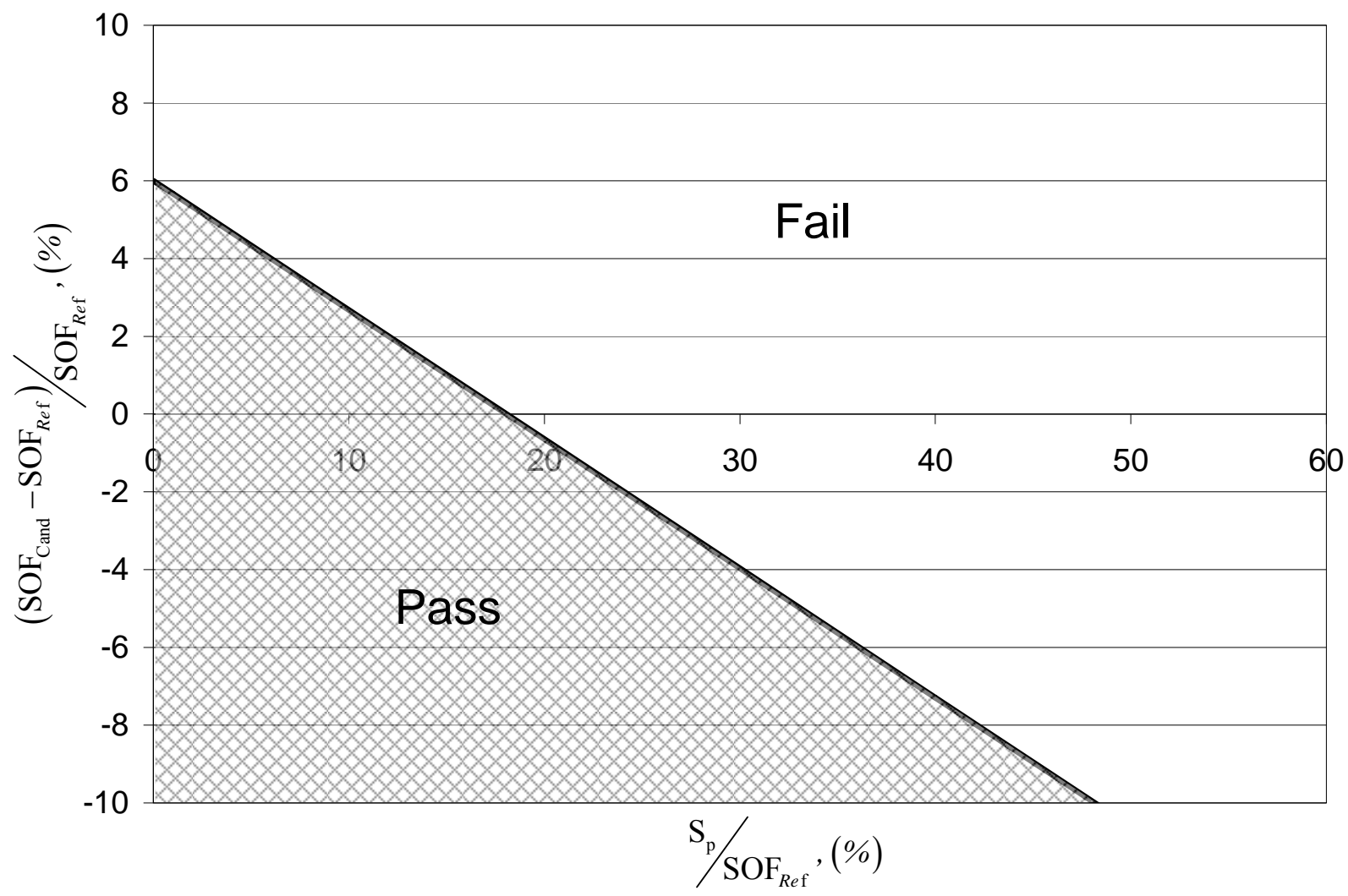

Figure 4- 1 Allowable SOF Variation for Passage

Figure 4- 2 shows the COV for each of the 52 sets extracted. Each of the labeled series represents the sets of FTP's tested shown in Appendix E (all other weight data is shown in Appendix F). The COVs range from $2.6 \%$ to $13.7 \%$, over a six month test period. Within this six month test period many test procedures were tried to reduce the COV. When the trials first started (series 1-23) a glass thimble was used with a porous porcelain bottom. When the extractor would cycle, then drain away the used solvent, the thimble would not drain quick enough to empty all of the used solvent. This was a problem because fresh solvent was unable to refill the thimble holding the filters and dissolve the organic fraction of the PM. This might have been a cause of higher variability because the filter wasn't experiencing a full cycle of extraction. This was solved by drilling six small holes in the porcelain bottom. This still allowed the filter to remain in the thimble, but would allow all used solvent to drain out of the thimble when the 
extractor cycled, insuring the filter would receive a full cycle of extraction. Another reason for variance in SOF results is human error between tests. Since working with a small weight difference for SOF ( $1 \mathrm{mg}$ extracted mass out of the 4 to $5 \mathrm{mg}$ net mass), any small error would result in a large variation in test results. A main problem that caused variation was folding of the filter sets. Even though many precautions were taken when folding such as wearing gloves, and sterilizing the folding utensils, errors can still occur from damaging the filters while folding, such as puncturing or tearing. This damage to the filter could result in weight loss before extraction leading to higher variation in test-to-test averages.

The 52 test series produced an overall average COV of $7.3 \%$. Included in the $7.3 \% \mathrm{COV}$ for the SOF, is the COV for all the repeat FTP tests, this is approximately $3 \%$ for PM for an early 1990's (5g/bhp-hr NOx, 0.25g/bhp-hr PM) electronically-controlled heavy-duty diesel engine and about $1.5 \%$ for a late 1990 's (4g/bhp-hr NOx, 0.1g/bhp-hr PM) electronically-controlled heavy-duty diesel engine. Excluding the COV from the PM, the COV for the SOF is as low as 4.3\%. Based on the variability of the extracted filter sets shown in Figure 4- 2, Additional studies were commissioned to determine the cause of the variability. 


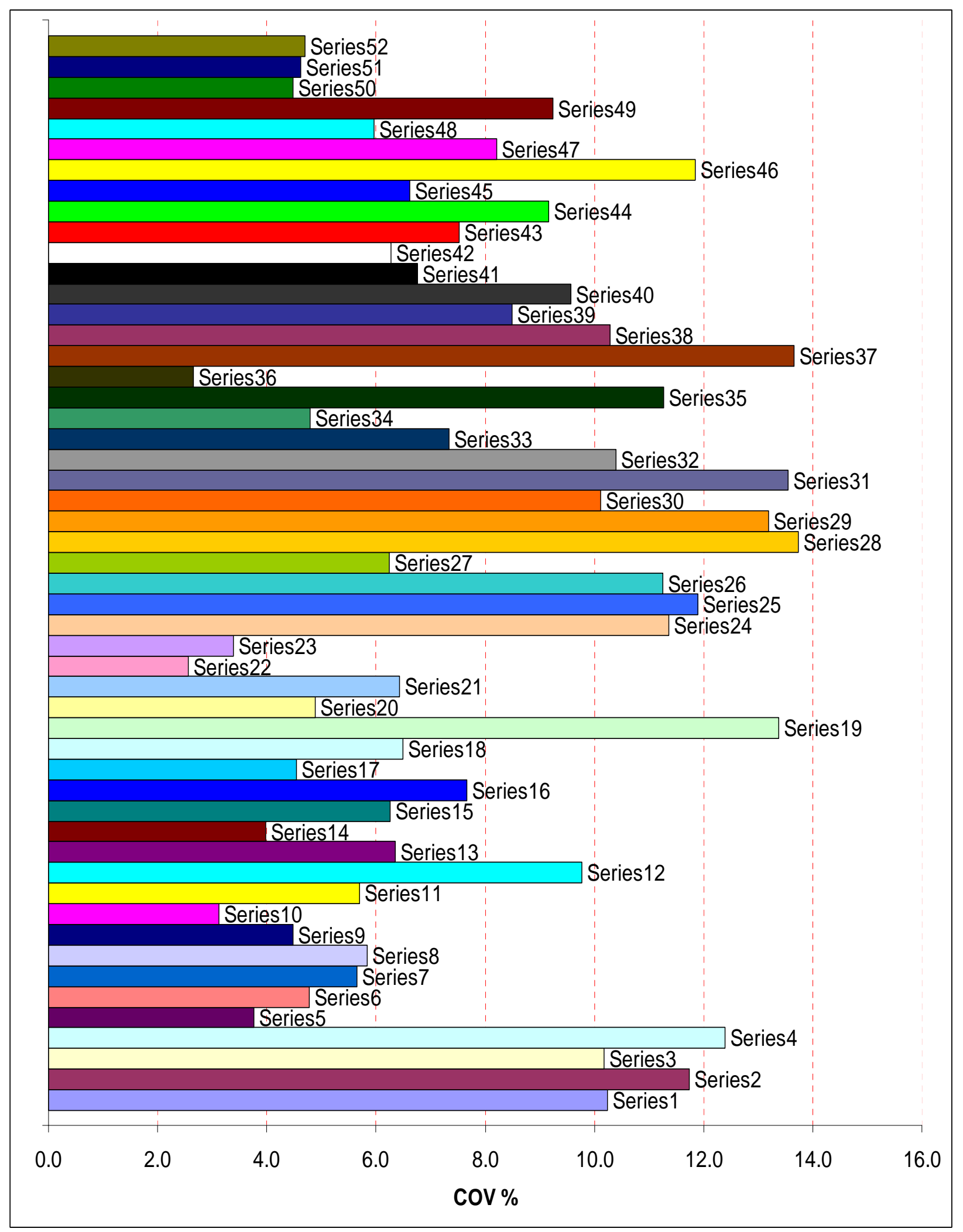

Figure 4- 2 COV of SOF for Each Set of FTPs PM filters Extracted 


\subsection{Unloaded Filter Weight Loss}

An examination of the weight loss of an unused, unloaded 70mm T60A20 filter pair illustrates that there is a significant weight loss from these filters as shown in Figure 4- 3. This weight loss from unloaded filters was a motivating factor that spurred this research effort. As illustrated in this figure, the net weight loss for the initial weight following the 11.5 hours extraction process $(7 / 8 / 08$ weighing) is on the order of $0.22 \mathrm{mg}$ for the five filter set examined. Based on prior work in the WVU CAFEE laboratory, this is a typical weight loss for unloaded dual 70mm T60A20 filters that are extracted using the Soxhlet procedures described herein. The variation (one standard deviation) in the five filter set examined for this research was $0.014 \mathrm{mg}$. However, a prior research program at CAFEE included a 16 unloaded extracted filter set with an average extracted mass of $0.19 \mathrm{mg}$ and a variation (one standard deviation) of $0.023 \mathrm{mg}$.

Although the average unloaded filter blank mass can be accounted for in the SOF calculation, the variability can not be accounted for and is detrimental to the passage criteria shown in Equations 4-3 and 4-4 and detailed above. For the typical case of $\sim 4 \mathrm{mg}$ net mass on the PM filter and $25 \%$ SOF ( $1 \mathrm{mg}$ extracted), the $0.023 \mathrm{mg}$ variation results in a $2.3 \%$ variation (one standard deviation) in the SOF just due to artifacts on the unused filter. It is assumed that these artifacts carry over after the filter set has been loaded with PM and are subsequently extracted. At a three standard deviation variation, this will result in an SOF variation of $\sim 7 \%$, a significant influence to the passage criteria as shown in Figure 4- 1. Based on this result, the minimum expected COV for the data presented is $2.3 \%$ due to filter media artifacts with this result supported by the data in Figure 4- 2 . 


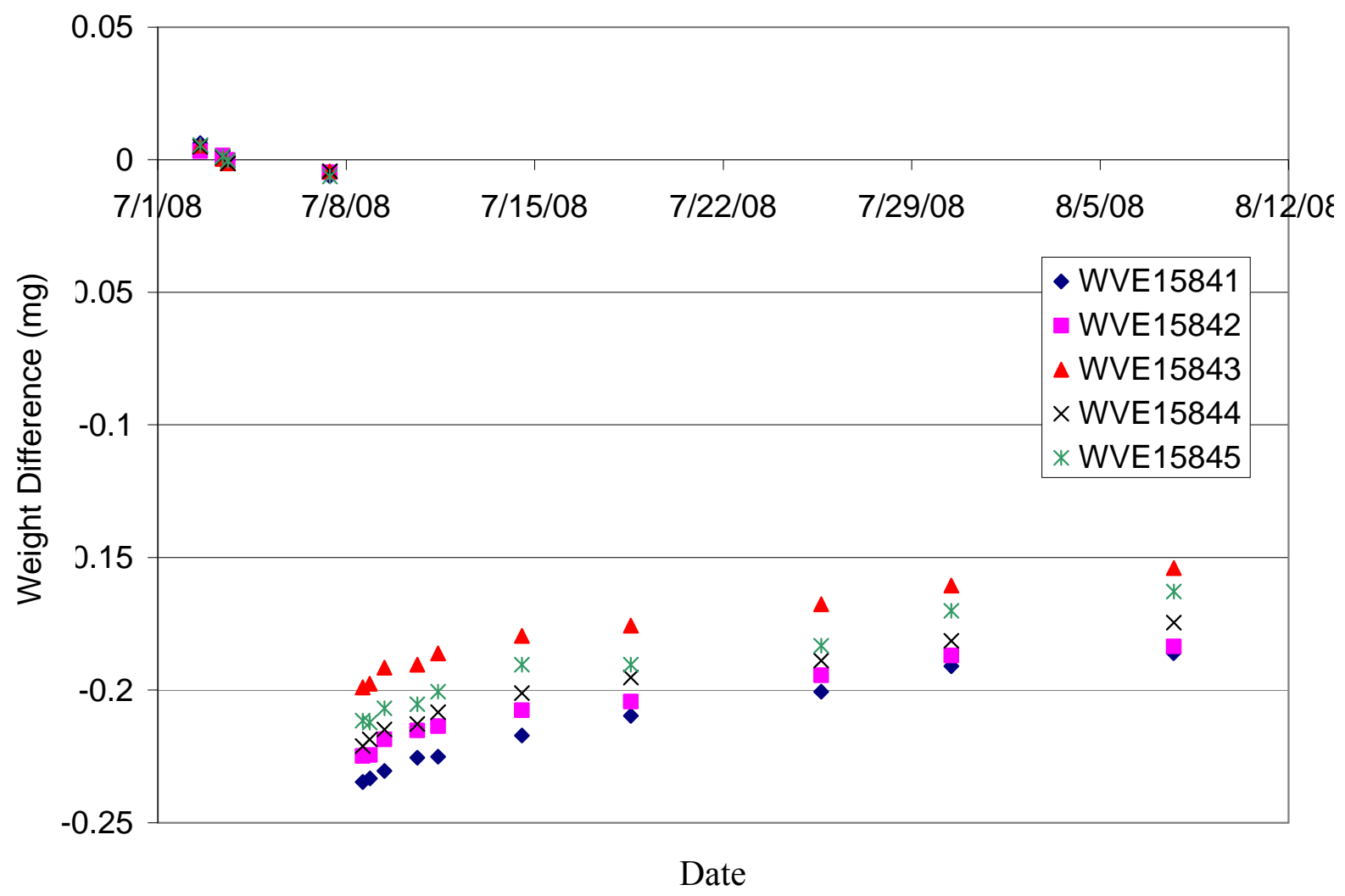

Figure 4- 3 Net weight loss of an unloaded filter due to Soxhlet extraction

\subsection{Weight Gain after Extraction}

Based on the weight gain of the extracted, unloaded filters in Figure 4- 3, additional experiments were performed to explore this effect. Used, loaded filters were examined and after extraction, the filters were left to sit in the clean room and weighed at regular intervals. After continual weighing of the filter sets, weight gains were again noticed. Figure 4- 4 shows the first three filters that were weighed over a period of six months. The filters gained on average of $0.40 \mathrm{mg}$ and have relatively the same slope of weight gain over time. This weight gain is ten times the allowable variation in the reference filter pair. Based on this finding, other tests were performed to try to find the cause of this weight gain. It is noted that good engineering practice would ensure that PM-loaded filters would be post weighed with 24 hours after a test and that 40 CFR Part 86, Subpart N requires post weighing within less than four days. However, some of 
the field work that CAFEE performs does result in post filter weighing to occur until a week or two after the tests have been performed, and the net mass could be influenced by a time delay between test and weighing. Additionally, extraction for SOF is not well defined in time requirements and it is possible that there could be significant delay between the post test weighing and SOF extraction.

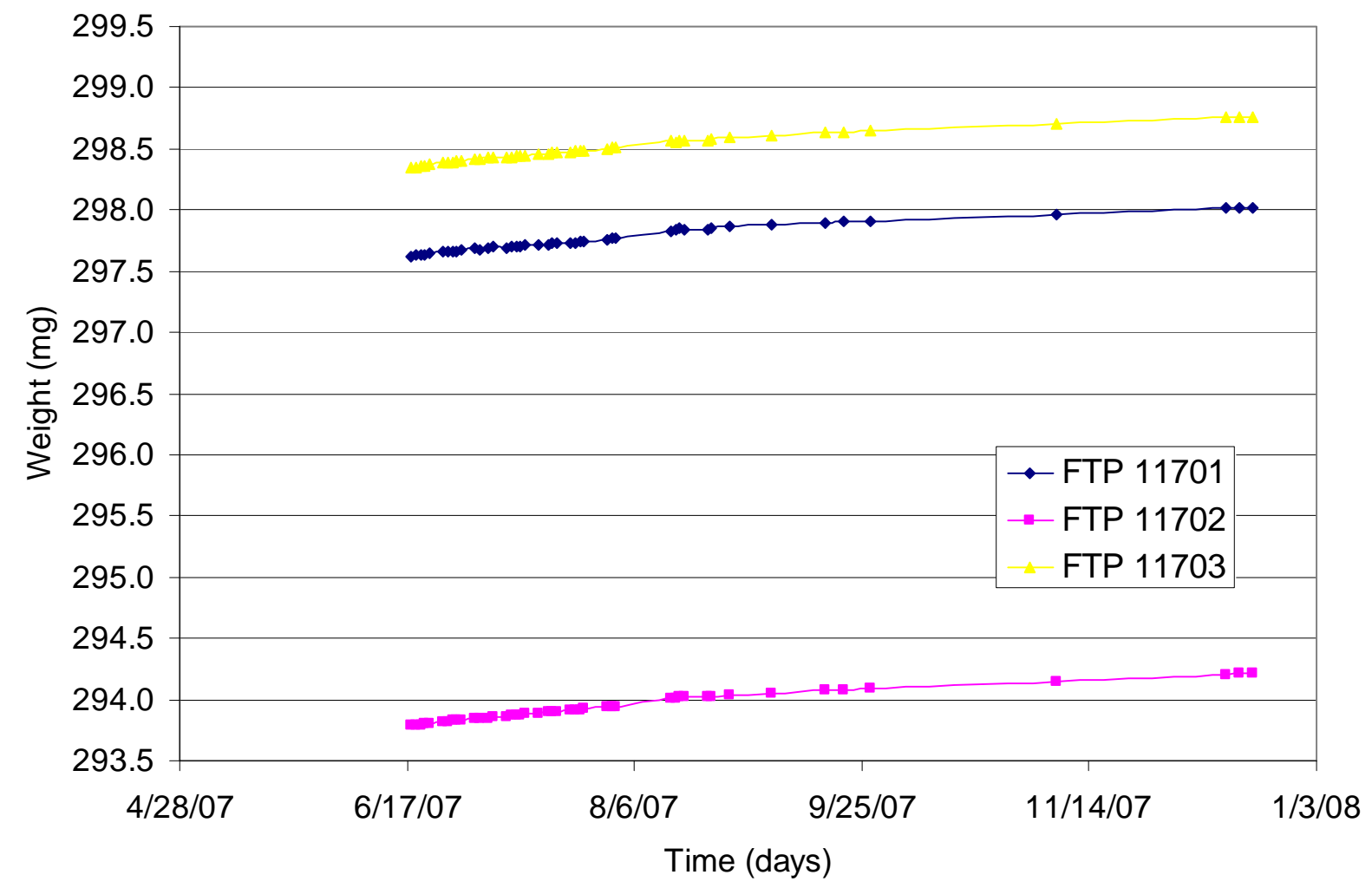

Figure 4- 4 Weighed Gained after Extraction over Time

This initial experiment was done without the use of a common set of reference filters. Because of the lack of the reference filters over the repeated weighing experiment, the experiment was then repeated with reference filters to track weight gain along with extracted filters to insure consistency of clean room conditions. For this subsequent set of experiments, unused T60A20 filter pairs were extracted. The interval for post weighing the extracted filters was three to five times the first day, then every day for the first week, then finally once a week 
for the remainder of the test period. In the discussion of the data, the average of the first four sets of mass measurement, for each filter set, were used as the baseline and then all measurements, for each filter set, were subtracted by this average measurement in order to provide a relative change in the filter mass for display purposes since the nominal mass on a filter pair could vary by tens of milligrams as illustrated in Figure 4- 4. Figure 4- 5 shows the reference filters used to tracks clean room variation. In this graph, the average and one standard deviation of multiple filters (five sets) are shown for two set of unused reference filters. The first set was filters kept in the clean room for the duration of this part of the study. The second set was folded, placed in the glass thimble, transported to the extraction room, and then transported back to the clean room. The reference filters gained negligible amounts of weight over the length of the experiment. This weight gain was on the order of the uncertainty of the scale.

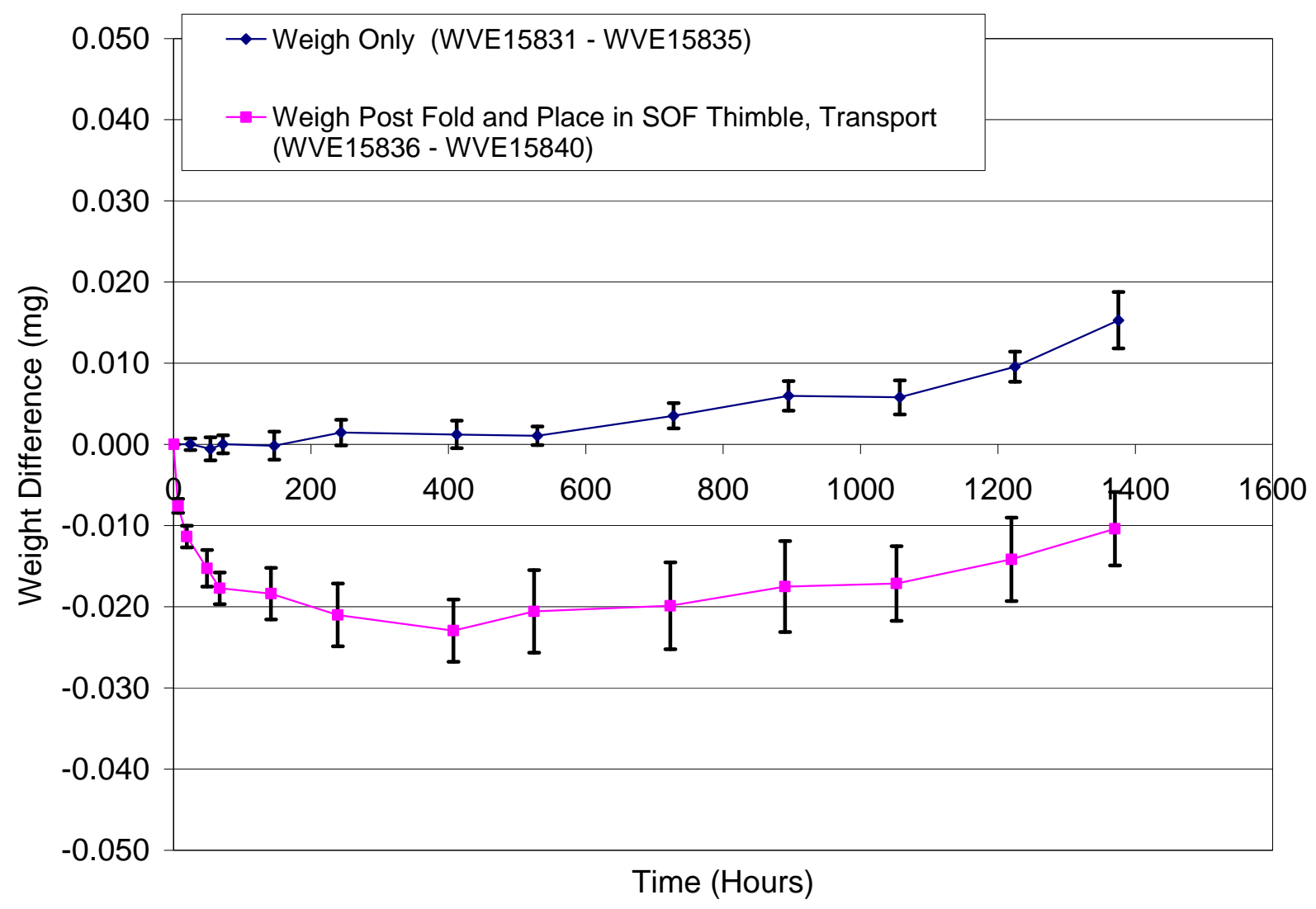

Figure 4- 5 Reference Filter Tracking 
Figure 4- 6 shows a steady increase in the post extracted weight of unloaded filters over a 31 day tracking period. The data is the average of the five filters and the bar represents one standard deviation of the weight gain for the five filters at each time. It is evident from this figure that an unloaded, extracted filter gains about $0.040 \mathrm{mg}$, the maximum variation of the reference filters, after three weeks of conditioning. Note these filters are the same filters as shown in Figure 4- 3 but with time and net filter mass set to zero for the initial weighing on $7 / 8 / 08$.

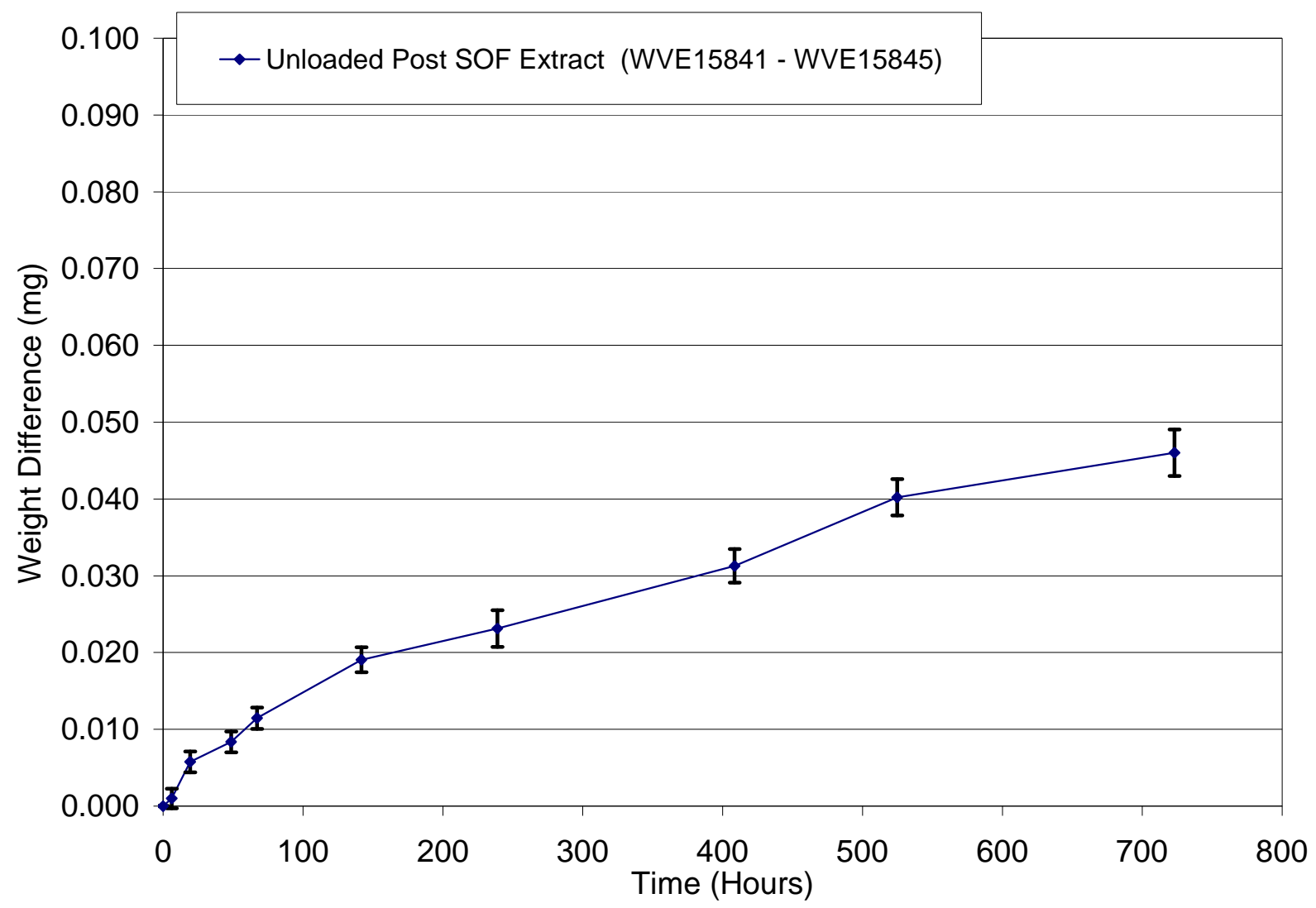

Figure 4- 6 Weight Gain of Unused T60A20 Extracted Filters Over 31 Days

\subsubsection{Oven Experiment}

A set of blank, unused filters were placed in an oven, heated to $375^{\circ} \mathrm{F}$ for one hour, and let cool in the clean room. These filters were then weighed at regular intervals to examine if water or volatile matter removed from the filters would affect weight gain. These filters lost 
$\sim 0.35 \mathrm{mg}$ after being placed in the oven and gained $\sim 0.15 \mathrm{mg}$ back over the 31 day interval as shown in Figure 4- 7. These filters gained over four times that of only extracted filters, showing that water and or volatiles in the filter itself could cause a large amount of weight gain. It is evident that T60A20 filters exposed to high temperatures will have sampling artifacts associated with them.

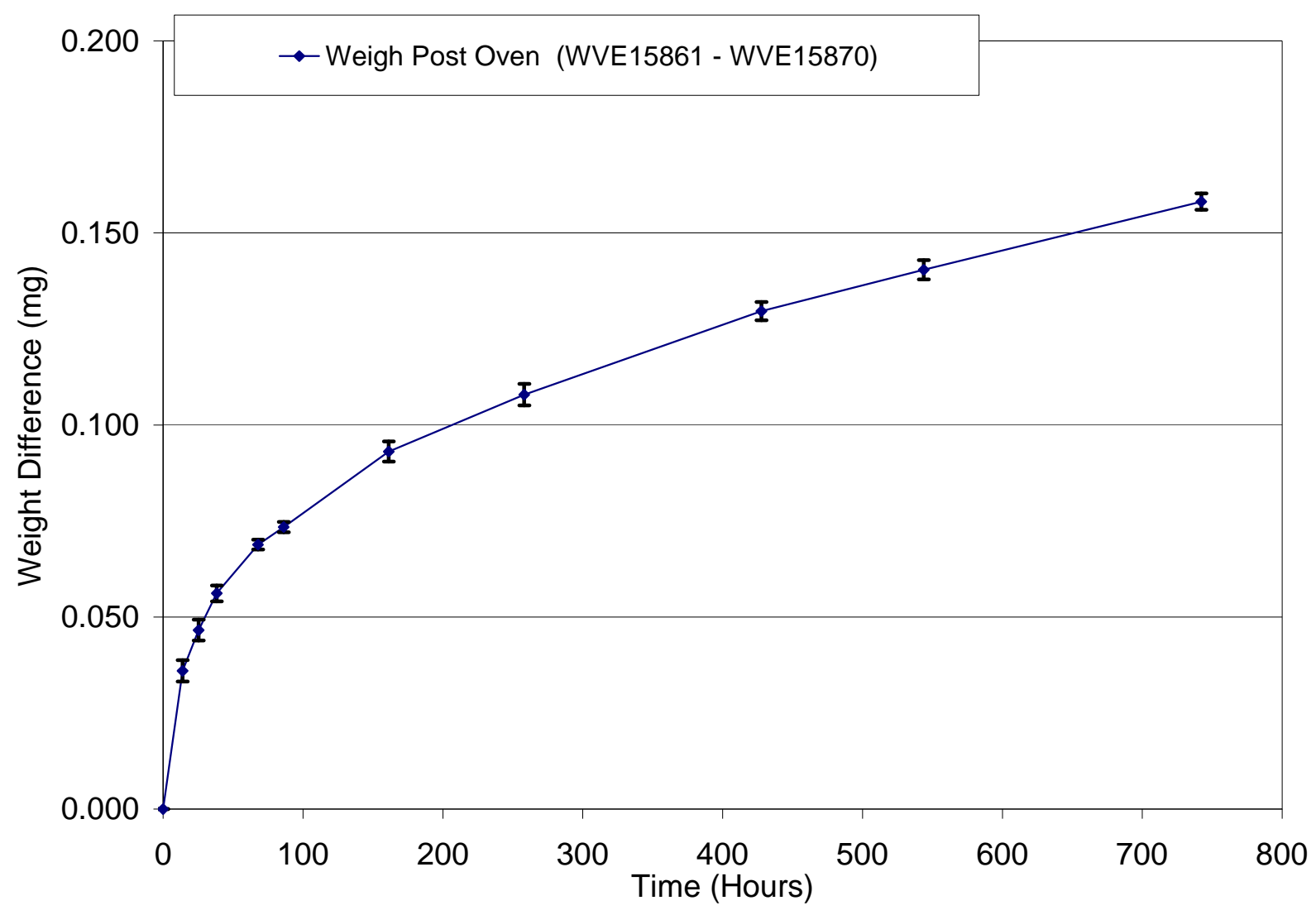

Figure 4- 7 Filters Backed in Oven

\subsubsection{Oil Deposit Experiment}

Another test was done to try to establish weight gain in the presence of organic matter. Unused filters were loaded with $\sim 1 \mathrm{mg}$ of 15 -W40 motor oil to simulate the amount of organic matter on a typical PM-loaded filter for the filters used in this work. This is supported by the 
data presented in Figure 2- 3 where the majority of the SOF is motor oil. These filters were placed in the clean room and weighed at regular intervals. As shown in Figure 4- 8, the oil deposited filters gained approximately $0.01 \mathrm{mg}$ over the 31 day test period. This is equivalent to the variation in the filter weighing, establishing that the presence of organic matter does not influence the change in weight over a one month duration.

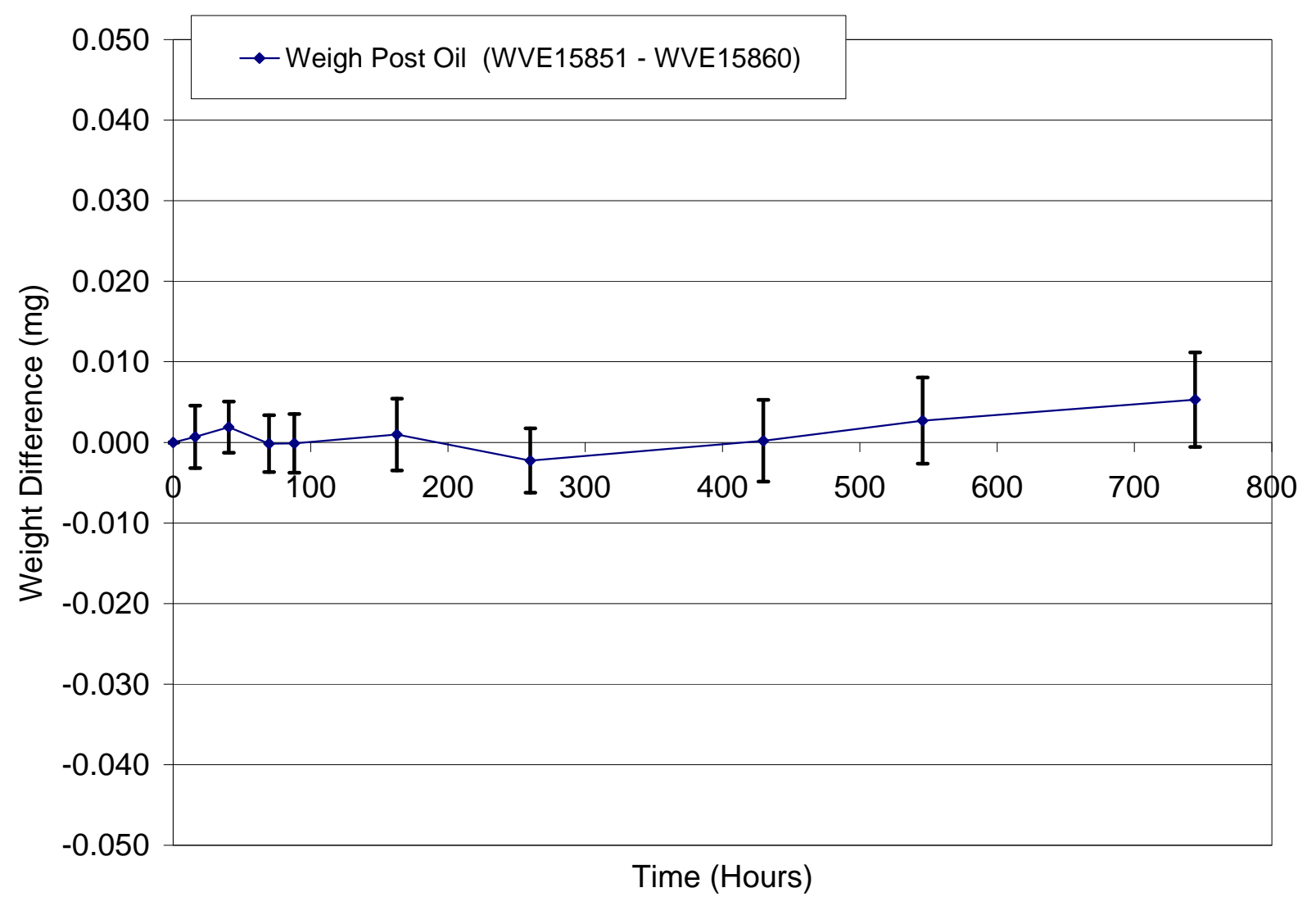

Figure 4- 8 Oil Deposited Filters

\subsubsection{Loaded vs. Non Loaded Filters}

The oil deposit experiment showed the weight gain in the filters is caused by something other than organic matter deposited on the filter during testing. The plots below illustrate two sets of filter groups. One set is blank, unused filters (unloaded) and displayed in Figure 4- 3 and 
Figure 4- 6; the other is loaded with PM from FTP testing (loaded). Both of these groups were extracted using the Soxhlet apparatus and stated testing procedures. After extraction these groups were placed in the clean room and weighed periodically for 31 days. Averages of the elapsed time between weighing and weight gained were calculated. From these averages, two linear results were plotted on a log-log graph. As seen in Figure 4- 9, both sets of filters produce relatively close slopes, 0.591 for loaded filter set, and 0.589 for unloaded filter set, showing for loaded and unloaded filter sets, post extraction weight gain over time occurs at the same rate. However, the intercept value is different. Based on the linear relationship on the log-log plot, the weight gain (W) of an extracted filter as a function of time ( $t$ ) has the form of the equation

$$
\mathrm{W}_{\text {gain }}=\mathrm{C}^{*} \mathrm{t}_{\text {hour }}{ }^{\mathrm{m}} \text {.Equation 4- } 6
$$

Where $\mathrm{C}$ accounts for the intercept and $\mathrm{m}$ is the slope of the line in log-log form. 


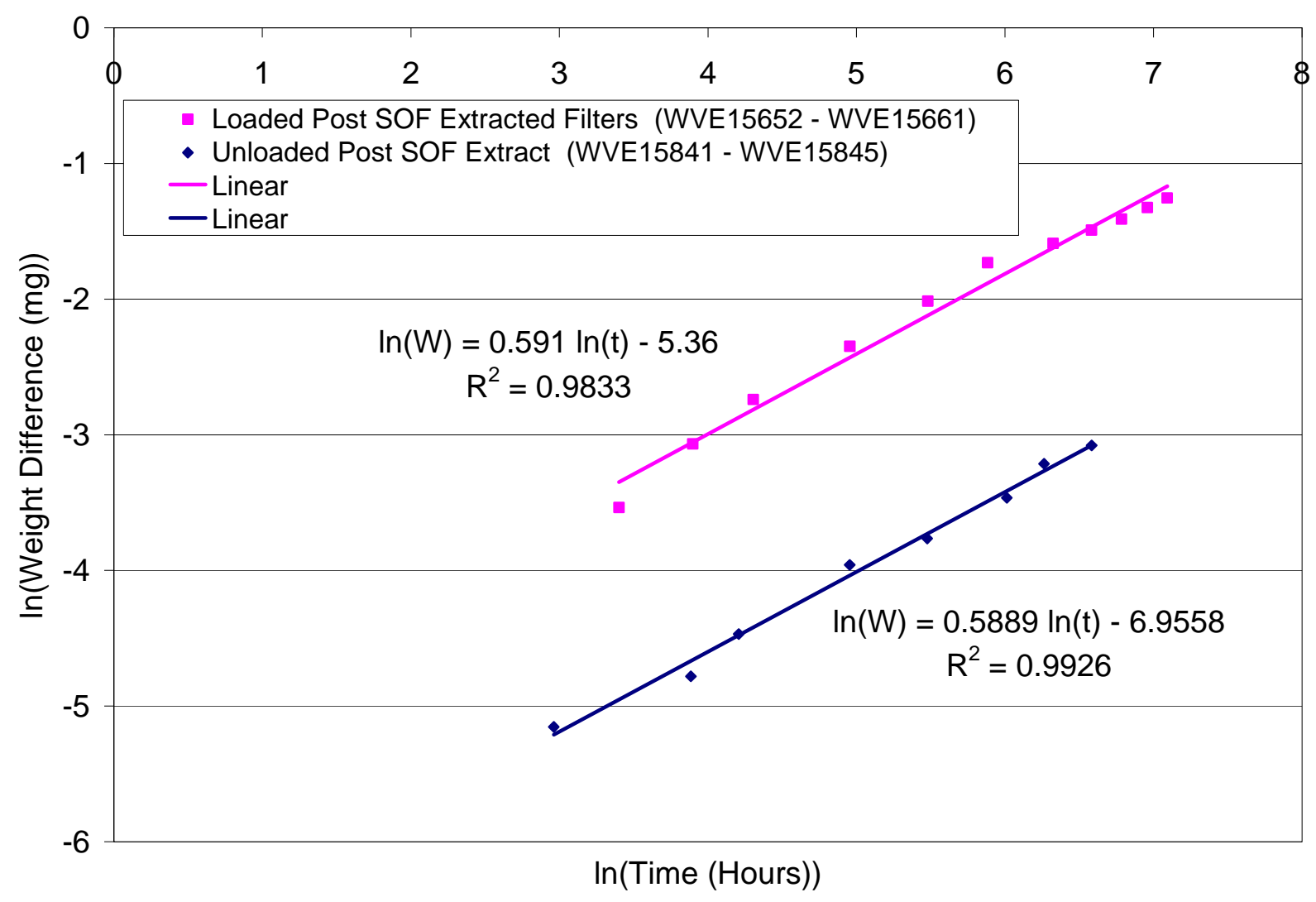

Figure 4- 9 Loaded vs. Unloaded Weight Gain

\subsubsection{Weight Gain Analysis}

Figure 4- 10 shows trend lines applied to each of the different extracted filter sets. The data for this plot is explained in more detail in section 4.5. For this plot only the weight gain over the test period is of value. The five sets of data show weight gain on a linear plot. This data is gathered from sets of similar filters that were averaged to create each curve for multiple filter sets. The bars represent one standard deviation of the data for that filter set. The data was then plotted as weight gain over time, and a trend line was applied to see the rate of growth for each filter set. Averaging these growth rates of the five data sets gives an average log-log slope of 0.740 regardless of the handling method of the filter. The method of freezing and shipping the filter in a Petri dish may result in greater post extraction weight gain than the other methods but additional testing would be warranted to make this claim. 


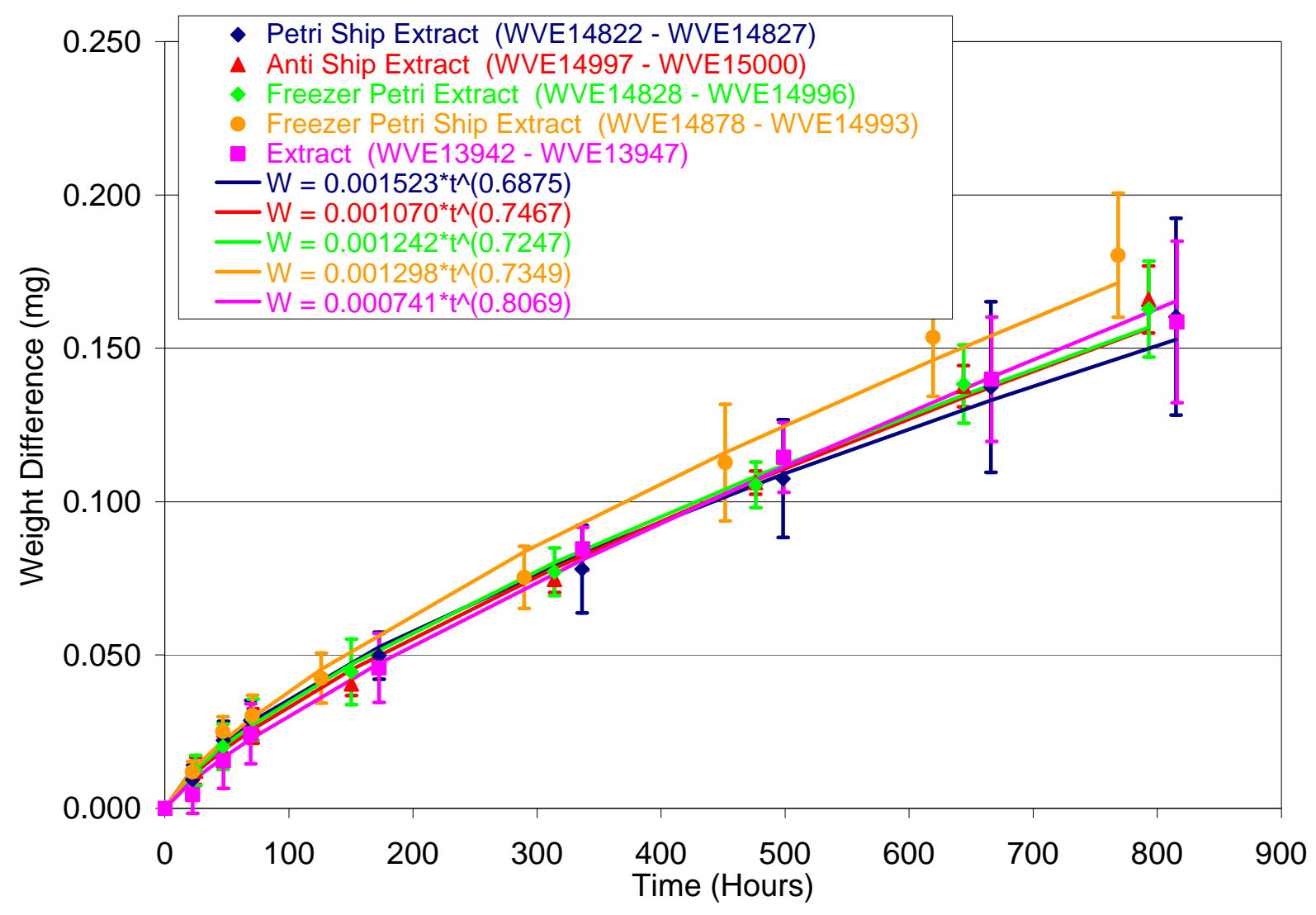

Figure 4- 10 Weight Gained after Extraction over Time

Based on the results presented in Figure 4- 10, a significant delay between post weighing and post extraction weighing can dramatically affect the SOF reading. Using the baseline of $\sim 4 \mathrm{mg}$ net PM mass and $\sim 1 \mathrm{mg}$ of extracted SOF from the filter, each $0.010 \mathrm{mg}$ weight gain results in a $1 \%$ error in the SOF determination. Therefore, post SOF extraction weighing should occur within the first 24 hours and should be consistent for all filters that are extracted.

\subsection{Handling of Filter Media}

The final objective of this study was to explore how transportation of the filter media would affect the variability of the Soxhlet extraction process and what impact it would have on 
overall weight gain. As shown in Table 3- 1, ten tests were performed to examine in detail how different ways of storing and shipping the filter media could affect the SOF.

Four tests were performed: for the first test loaded filters were placed in a Petri dish, boxed and shipped via Fedex to California and then returned. Once returned, a set of filters were extracted and a set were used for control. After extraction, both the control filters and extracted filters were periodically weighed for 31 days. The second test used loaded filters that were placed in 3M anti-static bags. The remainder of the test was performed the same as the first. The third and fourth test explored the option of freezing the filters before shipment. Media such as PM filters are typically stored in a freezer to minimize post test reactions that may occur before final extraction. All filters were placed in the freezer while being contained in Petri dishes. Once removed from the freezer, one set was boxed and shipped via Fedex to Califonia, while the other set went straight to extraction. For the third and fourth test, sets were also kept as controls. For these four tests another two sets of loaded filters were kept, one in the clean room only as a control, while the second set was extracted and then placed in the clean room. As in the loaded versus non-loaded filter experiments, the recorded data for the filters sets were averaged and referenced to zero average weight to obtain the resulting plots. Shown in Figure 4- 11 are the weight gain for the control set for each experiment. None of the control tests lost or gained over $.020 \mathrm{mg}$ in the 31 day test period, but each of the controls did show a similar pattern of loosing weight for the first 50 hours once placed back in the clean room after shipping. 


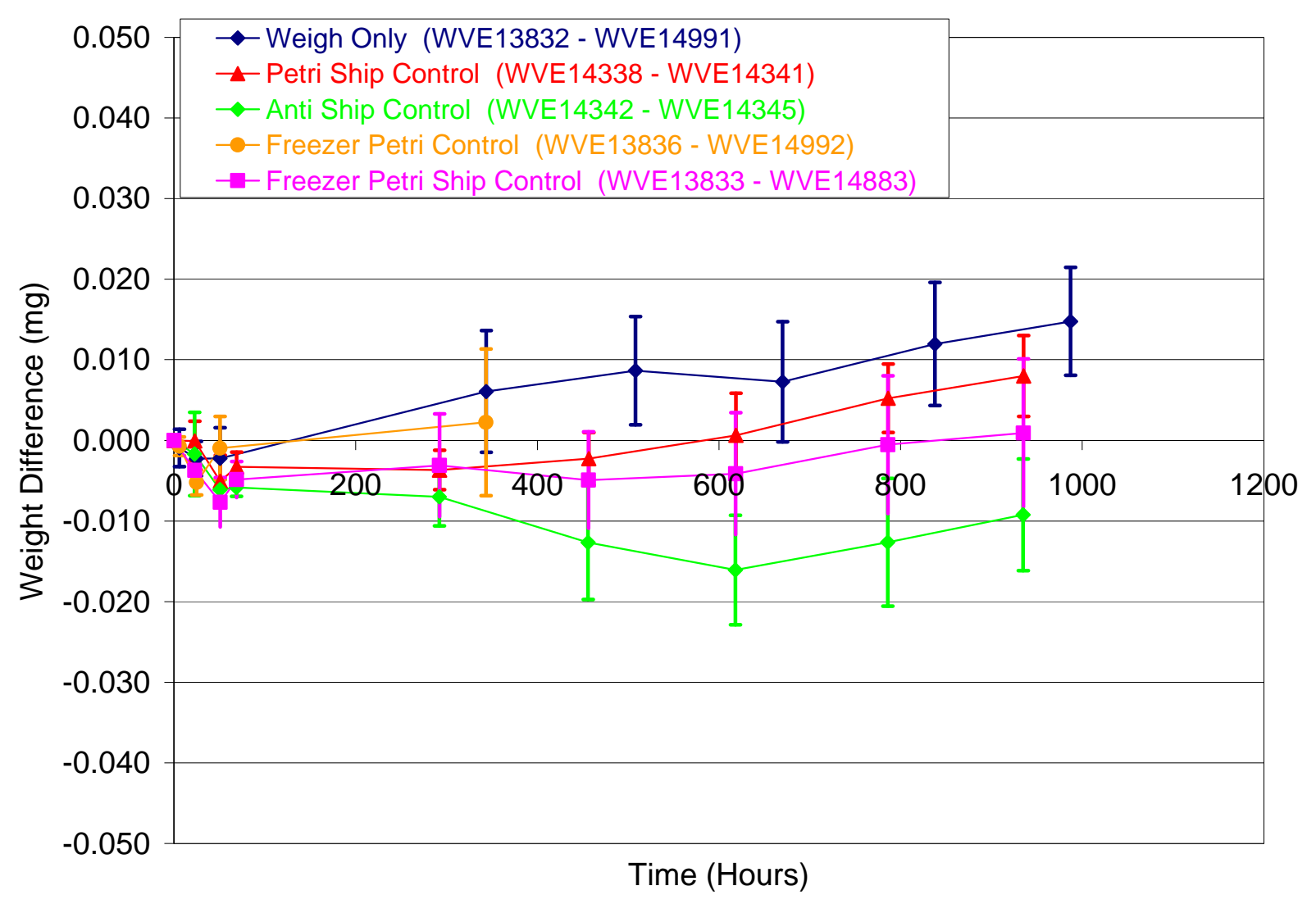

Figure 4- 11 Control Sets

Figure 4- 11 shows weight gain of averaged data from the shipping experiment appear to

be independent of the method used to store and ship the filters. These results show that the methods of shipping the filter sets examined in this work have little or no affect on weight gain after extraction for control filters. Weight gain occurs at close to the same rate for all sets recorded. 


\section{Conclusions and Recommendations}

\subsection{Conclusions}

This research was performed to solve three main objectives. First, develop a test procedure for SOF determination and quantifying the variability when using Soxhlet extraction. Second, examine how the SOF may vary over time when weighed in a controlled environment. Lastly, explore handling issues with the transportation of the filter media.

Test procedure "Determination of Soluble Organic Fraction (SOF) - CARB Method (SOP-0720)" (Appendix A) was created to provide guidelines to reduce results of test to test variation of Soxhlet extraction (Appendix B, Appendix C, Appendix D are referenced by Appendix A). Following this test procedure over a course of 52 test cycles, a COV was reached of $7.3 \%$. Removing the COV for all repeat FTP tests, a COV for Soxhlet extraction is approximately $4.3 \%$. Approximately half of this variation, $2.3 \%$, is due to artifacts on the filter media. These numbers are specific to the experiments conducted herein and should be used with care. These numbers are applicable to early 1990's HDDEs exercised over the FTP cycle. Based on Figure 4- 1, this permits the candidate fuel SOF value to be $\sim 4 \%$ higher than the reference fuel SOF value.

Weight gain after extraction is shown by loaded and unloaded filters sets, almost at the same rate, concluding that the accumulated weight is due to the filter itself, not from the PM on the filter. This weight gain happens after extraction of the filter, showing that the T60A20 filters are losing a percentage of its weight, which it continues to regain from atmospheric conditions when placed in a clean room. This weight gain can be expressed using the equation: $\mathrm{W}_{\text {gain }}=.001175\left(\mathrm{t}_{\text {hours }} 0.74014\right)$. 
Shipping and receiving of filter media had little affect on the weight gain of the filter sets. All shipping experiments examined provided almost identical rates of weight gain over the 31 days test cycle. Shipping and receiving filters did have a slight effect on the initial weighing of the filter media before extraction but is within the variation of the measurement for $70 \mathrm{~mm}$ T60A20 filter pairs. Each of the controls showed a slight decrease in weight before reaching a stable condition. This variation will result in increased variability of SOF.

\subsection{Recommendations}

The following suggestion will be made to expand on additional conclusions about this study. These recommendations are based on parameters that were not or could not be controlled in this work.

- When testing for SOF, the extraction device should be in close vicinity (within the same building) of the clean room to help reduce potential errors that can occur from transportation of filter media.

- The extraction device be placed in a controlled environment to keep atmospheric conditions close to that of clean room conditions and to insure no contaminates are introduced to the filters during extraction.

- A drying rack should be constructed to enable filters to dry in a flat state. This would facilitate quicker weighing.

- A measurement device should be constructed to assure continuous cycling of the extraction solvent in order to insure the number of cycles are reached for desired test ( 40 for this research).

- A more practical device should be constructed for folding filter sets to insure no damage to the filter is occurring. 
- Tests should be performed to determine the mechanism of weight loss and then weight gain of extracted, unloaded T60A20 filters.

- Tests should be performed to determine the mechanism of weight gain of extracted, loaded T60A20 filters. 


\section{References}

1. Clean Air Act, Environmental Protection Agency, Washington, DC, Feb. 24, 2004.

2. "Texas Low Emission Diesel (TXLED) Program," Texas Commission on Environmental Quality, 30 Sept. 2008. Accessed 19 Oct 2008 from <http://www.tceq.state.tx.us/implementation/air/ sip/cleandiesel.html\#top>.

3. "The California Diesel Fuel Regulations," California Air Resources Board, 14 Aug. 2004, California Code of Regulations. Accessed 19 Oct 2008 from <http://www.arb.ca.gov/fuels/iesel/ 081404dslregs.pdf>.

4. Code of Federal Regulations, CFR Title 40 Part 86-89, Office of Federal Register National Archives and Records Administration, Washington, DC, 2006.

5. Li, X., Chippior, L. W., and Gulder, L. O., "Effects of Cetane-Enhancing Additives and Ignition Quality on Diesel Engine Emissions," SAE Paper No. 972968, SAE International, Warrendale, PA, 1997.

6. ASTM D975-08, "Standard Specification for Diesel Fuel Oils," ASTM International, West Conshohocken, PA. See also www.astm.org.

7. "Hydrotreating," Sundyne Corporation World Headquarters, Arvada, Colorado, Accessed 9 Sep. 2008 from <http://www.sundyne.com/ind/details/0,,cli1_div92_eti8025,00+en-uss_0fsbc.html>.

8. Majewski, A. W., "Diesel Particulate Matter," DiesnetNet Technolgy Guide. 2002. Accessed 24 Apr. 2007 from <http://www.dieselnet.com/tech/dpm.html\#sof>.

9. "Toxicological Profile for Polycyclic Aromatic Hydrocarbons (PAHs)," U.S. Department of Health and Human Services, Public Health Service, Atlanta, GA, 1995.

10. Duran, A., Carmona, M., de Lucas, A., and Lapuerta, M., "Characterization of Soluble Organic Fraction in DPM: Optimization of the Extraction Method." SAE Paper 1999-01-3532, SAE International, Warrendale, PA, 1999.

11. Burtscher, H. and Majewski, A. W., "PM Measurement: Collecting Methods," DieselNet Technolgy Guide (2004). Accessed 24 Apr. 2007 from <http://www.dieselnet.com/tech/measure _pm_col.html>.

12. Ganzler, K. Salgo, A., and Valto, K., "Microwave Extraction: a Novel Sample Preparation Method for Chromatography," Journal of Chromatogrphy, Volume 1192, Issue 2, 30 May 2008, Pages 203207.

13. Soxhlet, F., "Die gewichtsanalytische Bestimmung des Milchfettes," Dinglers Polytechnisches Journal, 1879, Pages 462-465.

14. Masoori, G. A., "Supercritcal Fluids and Suprecritcal Fluid Extraction." Department of Chemical Engineering, University of Illinois at Chicago, Chicago, IL, 60680, Accessed 24 Apr. 2007 from <http://tigger.uic.edu/ - mansoori/SCF.and.SFE.by.TRL.at.UIC.pdf >

15. Kramer, B. K. and Ryan, P. B., "Soxhlet and Microwave Extraction in Determining the Bioaccessibility of Pesticides from Soil and Model Solids," Department of Chemistry, Emory University, Atlanta, GA, 2000. Accessed 24 Apr. 2007 from <http://www.p2pays.org/ref/ 15/14758.pdf>

16. Gomes, P. C. and Yates. D. A., "The Influence of Some Engine Operating Parameters on Particulate Emissions," SAE Paper 922222, SAE International, Warrendale, PA, 1992.

17. Flaim, K. A. C., "Study of Variations in Continuous Diesel Particulate Matter Size Measurements and Effect of Fuel Properties on DPM Size," Masters Thesis, Department of Mechanical and Aerospace Engineering, West Virginia University, Morgantown, WV, 2008. 
18. CAFEE Emissions Laboratory Standard Procedures, Calibration Procedures and Scheduled Maintenance Checks, Center for Alternative Fuels, Engines and Emissions, Version 20070305, Department of Mechanical and Aerospace Engineering, West Virginia University, Morgantown, WV, 2008..

19. "Sartorius SE2-F Ultra-microbalance," Precision Weight Balances, Bradford, MA, 2008.

20. "Test Method for Soluble Organic Fraction (SOF) Extraction," State of California Air Resources Board, Sacramento, CA, April 1989.

21. "Boiling Chip." Wikipedia, Wikimedia Foundation Inc, San Francisco, CA, 29 Oct 2008. Accessed 25 Sep 2008 from <http://en.wikipedia.org/ wiki/Boiling_chip>. 


\section{Appendix A \\ Determination of Soluble Organic Fraction (SOF) - CARB Method (SOP- 0720) \\ Overview}

The soluble organic fraction (SOF) is defined as the fraction of total particulate matter (TPM) that is removed in an extraction process from the TPM filter media collected from an emissions test. A Soxhlet extraction unit is used to cycle solvent through the test filter to remove the SOF fraction. The solvent removes the soluble hydrocarbon fraction from the TPM collected on the filter media, leaving the carbon fraction on the filter.

The procedures are based on $70 \mathrm{~mm}$ T60A20 filter media used for TPM collection of heavy-duty diesel engines exercised over the Federal Test Procedure (FTP) test schedule. These procedures may be used for other test cycles or other filter media. However, changes in solvent type, solvent volume, or cycle times may need to be varied and must be documented in the Extraction/Custody log (E/C log).

Precision scales are used to measure the filter mass before and after extraction.

\section{References}

40 CFR Parts 86.1312-88, 86.1339-90

State of California Air Resources Board, "Test Method for Soluable Organic Fraction (SOF) Extraction," April 1989.

SOP-0270 - "TPM Filter Weighing"

SOP-0600 - "TPM Media (70 mm) Handling"

SOP-0630 - "SOF Media Handling"

\section{Conditions}

Ensure that the TPM filter has been post weighed from the emissions test and that the TPM filter may be released for SOF analysis. The TPM filter pre- and post-test masses must be known to calculate the SOF fraction.

Filter media must be transferred between the Engine and Emissions Research Laboratory and the fume hood where the Soxhlet extraction resides. It is recommended to transfer the filters in their original Petri dishes if possible. Otherwise, the filter may be transported in the storage container that it is in. Ensure that the filter is secured to prevent any damage to the filter media or the loss of TPM from the filter.

The extraction is carried out using a toluene:ethanol binary solvent (32:68 wt/wt). Use only HPLC-grade toluene and ethanol to make this binary solvent.

\section{Procedure}

The following procedures parallel those of the 1989 ARB SOF document with minor differences.

1. Record the TPM filter pre-test and post-test mass in the E/C log. This may be obtained from the emissions test sheet log or from the filter weighing database. 
2. Ensure that the fume hood will be free of any other experiments for the duration of the extraction process. Ensure that each Soxhlet extraction unit is intact and free of any defects that may impede the performance of the extraction.

3. Ensure the area is clean of dust or any other foreign matter that may be deposited on the filters. Ensure that the stainless steel filter folder surface is clean of any foreign matter.

4. Ensure that the extraction unit has been rinsed with solvent prior to use. This may be accomplished by placing approximately $5 \mathrm{ml}$ of solvent in the extractor and another $5 \mathrm{ml}$ in the flask. The extractor, and then the flask, should be rotated or turned to coat the inside of each unit with the solvent. After the unit has been coated, the solvent is placed in the waste container. The extractor and flask are permitted to air dry in the fume hood while the rest of the procedure is performed.

5. Fold the filter in halves, quarters, and final eights with the particulate to the inside. Use the stainless steel filter forceps to fold the filter. Wear gloves to prevent transferring of hand oils or other contaminates to the forceps. However, do not touch the filters with your hands. If the filter is touched with any other surface other than the storage container, forceps, or folder surface, note this in the $\mathrm{E} / \mathrm{C} \log$. Avoid tearing fibers from the filter. If a fiber is torn from the filter, include the torn portion with the rest of the filter and note this in the $\mathrm{E} / \mathrm{C} \log$.

6. Place the folded filter in a Pyrex thimble, with the point of the fold towards the base of the thimble, and place the thimble in a Soxhlet extractor. Record the thimble ID on the $\mathrm{E} / \mathrm{C} \log$. Use care when inserting the thimble into the extractor to avoid damaging the extractor unit. Record the extractor ID on the $\mathrm{E} / \mathrm{C}$ log.

7. Place approximately $80 \mathrm{ml}$ of solvent in the flask. Use the bottle dispenser to set the volume of solvent to be transferred or use a $100 \mathrm{ml}$ beaker and measure to $80 \pm 2 \mathrm{ml}$.

8. Assemble the Soxhlet apparatus. Ensure that the extraction is done in the dark. If this is not possible, cover the Soxhlet apparatus with aluminum foil to keep light from the extractor unit

9. Turn on the water supply.

10. Turn on the electric heater units. Record the time in the $\mathrm{E} / \mathrm{C} \log$ that the heater was turned on.

11. Ensure that the cycle time is approximately 15 minutes. A minimum of 40 cycles are required or approximately eight and one half hours. It is recommended to use approximately eleven and one half hours for each extraction.

12. Turn off the heat and allow the solvent to stop boiling. Record the time in the E/C $\log$ that the heater was turned off.

13. Disassemble the Soxhlet apparatus and carefully remove the thimble. Dispose of the solvent from the flask and extractor unit into a waste container. Place the thimble on a clean surface in the fume hood and allow the solvent to evaporate for at least six hours. After six hours in the fume hood the filter can be placed back in the storage container and returned to the constant temperature and humidity clean room. Record the time in the $\mathrm{E} / \mathrm{C} \log$ that the filter was removed from the thimble. Record the time in the extraction $\log$ that the filter was placed in the clean room.

14. Follow the weighing procedures in SOP-0270 to record the post extraction filters mass. Record the time in the $\mathrm{E} / \mathrm{C} \log$ that the filter was weighed.

15. The loss of weight after this extraction is the SOF and is given by: 


$$
S O F=\frac{\text { TestMass }_{\text {final }}-\text { ExtractionMass }_{\text {final }}-\text { TestMass }_{\text {initial }}}{\text { TestMass }_{\text {final }}}
$$

Where the TestMassinitial is the mass of the filter set prior to the engine emissions test (Pre-Test Mass), TestMass final is the mass of the filter set after the engine emissions test (Post-Test Mass), and ExtractionMass is the mass of the filter set after the SOF extraction. Calculate and record the final SOF in the E/C log. The SOF may be corrected for background or interferences using good engineering judgment.

\section{Repeatability}

The recommended minimum net mass on a $70 \mathrm{~mm}$ filter set is $2 \mathrm{mg}$ for the FTP cycle. Lower net mass on a filter will result in higher variability in the SOF results from repeat emissions test (multiple filters extracted using the same engine and same conditions). For repeat FTP tests using the same engine and setup (engine set point file, fuel, intake and exhaust settings, etc.), the coefficient of variation (COV, standard deviation divided by the average) is approximately $3 \%$ for TPM for an early 1990's (5 g/bhp-hr NOx, $0.25 \mathrm{~g} / \mathrm{bhp}$-hr TPM) electronically-controlled heavy-duty diesel engine and about $1.5 \%$ for a late 1990's (4 g/bhp-hr NOx, $0.1 \mathrm{~g} / \mathrm{bhp}$-hr TPM) electronically-controlled heavy-duty diesel engine. The extraction process adds additional variation to the final SOF value and for tightly-controlled conditions the overall SOF variation should have a COV of less than $20 \%$. Filters with higher TPM loading will generally have lower COV values.

\section{Interferences}

It is possible that organic matter existed on the filter prior to the emissions test. It is recommended that pre-cleaned filter be used prior to the emissions test. Additionally, torn or missing filter media will lead to incorrect SOF determination. Any abnormality with the filter or $\mathrm{SOF}$ extraction process must be reported on the $\mathrm{E} / \mathrm{C} \log$.

Field blanks must be extracted as part of the SOF analysis and may be used to correct the SOF value. Likewise, tunnel background filters should also be extracted and used in the correction of the SOF value. Similar filters (lot number) that were assigned for the emissions must be extracted during the same time period as the test filter SOF extractions. Field blanks should be at least $10 \%$ of the TPM test filters with a minimum of 6 filter sets per test program.

\section{Corrective Actions}

If there were any problems, record the issue in the $\mathrm{E} / \mathrm{C} \log$ and contact the principal investigator and laboratory supervisor.

Outliers should be identified using the methodology stated in the test plan.

Filter sets from repeat tests with a large COV may be re-extracted a second time to determine if the initial extraction process failed to remove all of the organic matter. The principal investigator must approve the second extraction. If a filter set is extracted a second time, both the initial and final extraction values are to be reported with the dataset. 


\section{SOF Media Handling (SOP-0630) \\ Overview}

\section{Appendix B}

The laboratory's $70 \mathrm{~mm}$ TPM media may be used for the SOF determination. The procedures used in the TPM handling must be followed. Additional handling for the SOF determination is given below for filters shipped to external laboratories. Filter analyzed for SOF internally at WVU do not need to follow this procedure.

\section{References}

None

\section{Conditions}

The media must be tracked using the existing TPM software. After post TPM weighing, the used media is placed in anti-static bags if sent for analysis to an external laboratory. Tweezers must be used to handle the filters. Under no circumstances shall filters come in contact with any surfaces other than the storage container, tweezers, or filter holders. If the filter contacts any other surface, the filter must be marked as invalid.

\section{Procedure - Check In}

1. Follow the TPM filter weighing and handling procedures.

\section{Procedure - Use}

1. Follow the TPM filter use procedures.

2. After the TPM filter post weighing, place the used media in the storage container. Maintain custody sheets with the media.

\section{Procedure - Check Out}

1. Check out should occur as late in the day as possible so that the shipping container does not sit at the shipping company for an extended period of time. Additionally, the day that the shipping occurs should be done so that the analytical laboratory receiving the media is open the next morning.

2. When the media is to be shipped, check the media out with the Media Custody Log program. Use dust-free gloves to handle the media container. Visually inspect the media to ensure that there is no apparent damage. Contact the principle investigator if there are problems.

3. The media must be secured to prevent movement of the filter media as it is being shipped. Package the media into anti-static bags. Label each anti-static bag with the same filter identification as that on the Petri dish. Tape the anti-static bag onto a sheet of paper. Up to four anti-static bags may be taped to one sheet of paper. Multiple sheets of paper may be stacked together. Place the sheets together between two pieces of cardboard and tape the cardboard together. Cover the cardboard in "bubble wrap" or similar material. Place contents in a shipping box. Ensure that the contents are not free 
to move in the box. Place the media custody sheets in the box with the media. Other media samples should be shipped in a different box.

4. Take the box to the local shipping office (FedEx). Do not allow the box to sit in the open environment where the media is exposed to high temperatures.

\section{Corrective Actions}

If the filter contacts any other surface other than the media storage containers, tweezers, or filter holder, the filter must be identified on the custody sheet and the principal investigator notified. Any filter that is damaged prior to use must not be used. Any filter that is damaged during or after use must be clearly noted in the logs. Filters damaged prior to the end of the test phase in which an extra set of filters can not be used in its place may result in the test being repeated. 


\section{Appendix C}

TPM Filter Weighing (SOP-0270)

Overview

Precision scales are used to measure filter weights. Environment, calibration and weighing information is recorded by software on the weighing room computer.

\section{References}

40 CFR Parts 86.1312-88, 86.1339-90

\section{Conditions}

Ensure that the environmental conditions of the weighing and storage area within the following specifications.

- Filters must be exposed to the environment of the conditioning area for a minimum of 1 hour prior to weighing.

- Filters must be stored in covered Petri dishes or sealed filter holders in the conditioned storage area when not in use.

- Used filter pairs must be stored face-to-face in covered Petri dishes.

- If the sample on the filters contacts the Petri dish or any other surface, the associated test is void and must be re-run.

- At least two unused reference filter pairs must be in use at any given time. If the average weight of the reference filter pairs changes between sample filter weighings by more than 40 micrograms, then all the associated sample and background filters being stabilized are void and their respective emissions tests must be re-run.

- The 1988 version requires post weight before 56 hours.

- Filters must be weighed within 4 hours of reference filters but preferably during the same session.

- The reference filters should be changed once a month, however, reference filters must not be changed until all filters being processed with those reference filters are pre- and post-weighed

- The zero/tare drift of the scale must not exceed \pm 5 micrograms during a weighing session and must be checked after weighing 10 filters and at the conclusion of a weighing session.

The filter weighing room must be maintained at the following conditions

- Temperature: $22 \pm 3^{\circ} \mathrm{C}$

- Dew Point: $9.4 \pm 3^{\circ} \mathrm{C}$

- Relative Humidity: $45 \pm 8 \%$

where the dew point and relative humidity are averaged over 10 minutes. If these conditions are not met the room conditions must be brought to within the above specifications and allowed to remain in that state for one hour prior to performing filter weighing operations.

\section{Procedure}

1. Check that the room conditions are within specification.

2. Level the scale. 
3. Follow the manufacturer's recommended procedures for the scale's internal calibration or adjustments.

4. Check that the zero/tare reading is $0 \pm 5$ micrograms. If the zero/tare reading is outside this range, re-zero the scale. Record the zero/tare reading from the scale.

5. Place the $200 \mathrm{mg}$ reference weight on the balance and record the reading. The weight must be within \pm 5 micrograms of its actual value. For the "CAL4" weight, that value is $199.992 \mathrm{mg}$.

6. Remove the $200 \mathrm{mg}$ reference weight and check that the zero/tare reading is $0 \pm 5$ micrograms. If the zero/tare reading is outside this range, re-zero the scale, re-weigh the $200 \mathrm{mg}$ reference weight and re-check the zero until both the \pm 5 microgram zero/tare and \pm 5 microgram reference weight conditions are satisfied.

7. Measure and record the weights of the reference filter pairs.

8. Check that the difference in the sum of the weight of the each reference filter with their previously recorded value is within 40 micrograms.

9. Check that the zero/tare reading is $0 \pm 5$ micrograms. If the zero/tare reading is outside this range, re-zero the scale, re-weigh the reference filter pairs.

10. Measure and record the weights of the sample filters.

11. After 10 filter sets have been weighed, check that the zero/tare reading is $0 \pm 5$ micrograms. Record the zero/tare reading from the scale. If the zero/tare reading is outside this range, re-zero the scale and re-weigh the previous filters since that last valid zero/tare reading.

12. Continue with steps 10 and 11 until the entire filter set has been weighed or four hours has passed since the reference filter pairs were weighed.

\section{Corrective Actions}

If the room conditions can not be met, wait until the room conditions are within specifications. If the scale is unable to meet the zero, spans, or reference filter requirements, ensure that the room conditions are stable. If problems persist, contact the laboratory supervisor. 


\section{Appendix D}

TPM Media (70 mm) Handling (SOP-0600)

\section{Overview}

The laboratory's $70 \mathrm{~mm}$ filters are used for the TPM determination. The filters must be preconditioned and pre-weighed before use.

\section{References}

40 CFR Parts 86.1312-88, 86.1339-90

\section{Conditions}

The filters must be pre-weighed before using. Tweezers must be used to handle the filters. Under no circumstances shall filters come in contact with any surfaces other than the storage containers, tweezers, or filter holders. If the filter contacts any other surface, the filter must be marked as invalid. Filters are to be loaded and unloaded in the PM room pass thru window only.

\section{Procedure - Check In}

1. Obtain the required number of filters for the test session.

2. Record each filter as "Pre-use" in the PM room database.

3. Attach PM filter bar code for each filter set on the test sheet.

4. Place Petri dish(s) and test sheet(s) in the filter room pass thru window.

\section{Procedure - Use}

1. Install a filter into a filter holder in the pass thru window.

2. Place a cover over the entrance to the filter holder to prevent dirt or debris from entering the filter holder.

3. Remove the filter holder from the PM room and take to the secondary dilution tunnel.

4. Remove the PM filter holder in the secondary tunnel, if installed. Remove the cover from the new PM filter holder and place over the old PM filter holder. Install the new PM filter holder into the secondary tunnel.

5. Return the old PM filter holder to the PM room pass thru window. Remove the filters from the filter holder. If filters are dummy media, discard filters. If filters are from a test, return the filter to the appropriate Petri dish.

6. At the end of the last test, place test sheets in PM room pass thru window.

\section{Procedure - Check Out}

1. Retrieve Petri dish(s) and test sheet(s) from the filter room pass thru window.

2. Record each filter as "Post-use" in the PM room database.

3. Place Petri dishes on shelves to condition for at least one hour. Remove Petri dish lid to permit air movement around the filter during conditioning.

4. Follow TPM Filter Weighing procedure for post weighing the filters.

5. After post weighing procedures, the filter may be stored in a freezer if further analyses are required; ensure that custody sheets remain with each filter set. If filters are not required to be maintained, discard used filters. 


\section{Corrective Actions}

If the filter contacts any other surface other than the media storage containers, tweezers, or filter holder, the filter must be marked as invalid. Any filter that is damaged prior to use must not be used. Any filter that is damaged during or after use must be clearly noted in the logs. Filters damaged prior to the end of the test phase in which an extra set of filters can not be used in its place will result in the test being repeated. 


\section{Appendix E}

\begin{tabular}{|c|c|c|c|c|c|c|c|c|c|c|c|c|c|}
\hline & \multicolumn{13}{|c|}{ Double $70 \mathrm{~mm}$ Only } \\
\hline & $\begin{array}{c}\text { Run } \\
\text { Number }\end{array}$ & $\begin{array}{c}\text { Test } \\
\text { Number }\end{array}$ & $\begin{array}{c}\text { Extractor } \\
\text { Used }\end{array}$ & $\begin{array}{c}\text { Thimble } \\
\text { used }\end{array}$ & Date Ran & Preweight & Postwieght & $\begin{array}{c}\text { PM } \\
\text { Amount }\end{array}$ & $\begin{array}{l}\text { Sox } \\
\text { Post }\end{array}$ & $\begin{array}{c}\text { Sox } \\
\text { Postweight }\end{array}$ & Sox Diff & SOF & $\operatorname{cov} \%$ \\
\hline \multirow[b]{3}{*}{ Series 1} & 11657 & & 6 & & $6 / 4 / 07$ & 293.4930 & 298.4053 & 4.9123 & $6 / 21 / 07$ & 297.7696 & 0.6357 & 12.9410 & 10.2 \\
\hline & 11660 & & 1 & & $6 / 12 / 07$ & 291.8482 & 296.8787 & 5.0305 & $6 / 21 / 07$ & 296.1602 & 0.7185 & 14.2829 & \\
\hline & 11663 & & 4 & & $6 / 4 / 07$ & 293.1744 & 298.1870 & 5.0126 & $6 / 21 / 07$ & 297.604 & 0.5830 & 11.6307 & \\
\hline & & & & & & & & & & & & & \\
\hline \multirow[b]{3}{*}{ Series 2} & 11666 & & 3 & & $6 / 4 / 07$ & 293.4063 & 298.3954 & 4.9891 & $6 / 7 / 07$ & 297.6877 & 0.7077 & 14.1849 & 11.7 \\
\hline & 11667 & & 2 & & $6 / 4 / 07$ & 284.6155 & 289.7425 & 5.1270 & $6 / 7 / 07$ & 288.9848 & 0.7577 & 14.7786 & \\
\hline & 11668 & & 1 & & $6 / 4 / 07$ & 296.0604 & 301.0032 & 4.9428 & $6 / 7 / 07$ & 300.1336 & 0.8696 & 17.5933 & \\
\hline \multirow[b]{4}{*}{ Series 3} & & & & & & & & & & & & & \\
\hline & 11674 & & 1 & & $6 / 6 / 07$ & 294.4327 & 298.9501 & 4.5174 & $6 / 7 / 07$ & 298.1249 & 0.8252 & 18.2671 & 10.2 \\
\hline & 11675 & & 2 & & $6 / 6 / 07$ & 293.8072 & 298.3485 & 4.5413 & $6 / 7 / 07$ & 297.6723 & 0.6762 & 14.8900 & \\
\hline & 11676 & & 2 & & $6 / 12 / 07$ & 288.9654 & 293.5200 & 4.5546 & $6 / 13 / 07$ & 292.7618 & 0.7582 & 16.6469 & \\
\hline & & & & & & & & & & & & & \\
\hline \multirow[b]{3}{*}{ Series 4} & 11679 & & 1 & & $6 / 13 / 07$ & 294.8682 & 299.0822 & 4.2140 & $6 / 14 / 07$ & 298.3118 & 0.7704 & 18.2819 & 12.4 \\
\hline & 11680 & & 2 & & 6/13/07 & 287.5177 & 291.8069 & 4.2892 & $6 / 14 / 07$ & 291.0326 & 0.7743 & 18.0523 & \\
\hline & 11681 & & 3 & & $6 / 13 / 07$ & 291.2214 & 295.5857 & 4.3643 & $6 / 14 / 07$ & 294.6097 & 0.9760 & 22.3633 & \\
\hline & & & & & & & & & & & & & \\
\hline \multirow[b]{3}{*}{ Series 5} & 11683 & & 3 & & $6 / 12 / 07$ & 291.7609 & 296.1001 & 4.3392 & $6 / 21 / 07$ & 295.5792 & 0.5209 & 12.0045 & 3.8 \\
\hline & 11684 & & 4 & & $6 / 12 / 07$ & 294.4005 & 298.6851 & 4.2846 & $6 / 21 / 07$ & 298.1593 & 0.5258 & 12.2719 & \\
\hline & 11685 & & 6 & & $6 / 12 / 07$ & 295.4195 & 299.8262 & 4.4067 & $6 / 21 / 07$ & 299.2572 & 0.5690 & 12.9122 & \\
\hline & & & & & & & & & & & & & \\
\hline \multirow{4}{*}{ Series 6} & 11701 & & 1 & & $6 / 15 / 07$ & 293.8455 & 298.2144 & 4.3689 & $6 / 18 / 07$ & 297.6211 & 0.5933 & 13.5801 & 4.8 \\
\hline & 11702 & & 2 & & $6 / 15 / 07$ & 290.0210 & 294.3744 & 4.3534 & $6 / 18 / 07$ & 293.7855 & 0.5889 & 13.5274 & \\
\hline & 11703 & & 3 & & $6 / 15 / 07$ & 294.5894 & 298.9936 & 4.4042 & $6 / 18 / 07$ & 298.3459 & 0.6477 & 14.7064 & \\
\hline & & & & & & & & & & & & & \\
\hline \multirow[b]{3}{*}{ Series 7} & 11778 & & 1 & & $6 / 21 / 07$ & 296.1069 & 300.6263 & 4.5194 & $6 / 21 / 07$ & 299.858 & 0.7683 & 17.0000 & 5.7 \\
\hline & 11779 & & 2 & & 6/21/07 & 296.5233 & 300.9909 & 4.4676 & $6 / 21 / 07$ & 300.2569 & 0.7340 & 16.4294 & \\
\hline & 11780 & & 3 & & $6 / 21 / 07$ & 293.6939 & 298.1681 & 4.4742 & $6 / 21 / 07$ & 297.348 & 0.8201 & 18.3295 & \\
\hline & & & & & & & & & & & & & \\
\hline \multirow[b]{3}{*}{ Series 8} & 11783 & & 4 & & $6 / 21 / 07$ & 297.1851 & 301.6694 & 4.4843 & $6 / 21 / 07$ & 300.9424 & 0.7270 & 16.2121 & 5.8 \\
\hline & 11784 & & 5 & & $6 / 21 / 07$ & 295.6151 & 300.2353 & 4.6202 & $6 / 21 / 07$ & 299.4021 & 0.8332 & 18.0339 & \\
\hline & 11785 & & 6 & & $6 / 21 / 07$ & 296.9137 & 301.4708 & 4.5571 & $6 / 21 / 07$ & 300.655 & 0.8158 & 17.9017 & \\
\hline & & & & & & & & & & & & & \\
\hline \multirow{4}{*}{ Series 9} & 11878 & & 1 & & $6 / 22 / 07$ & 293.2093 & 299.3287 & 6.1194 & $6 / 25 / 07$ & 298.2403 & 1.0884 & 17.7861 & 4.5 \\
\hline & 11879 & & 2 & & $6 / 22 / 07$ & 290.3185 & 296.4015 & 6.0830 & $6 / 25 / 07$ & 295.2868 & 1.1147 & 18.3248 & \\
\hline & 11880 & & 3 & & $6 / 22 / 07$ & 294.1768 & 300.1747 & 5.9979 & $6 / 25 / 07$ & 299.0103 & 1.1644 & 19.4135 & \\
\hline & & & & & & & & & & & & & \\
\hline \multirow[b]{3}{*}{ Series 10} & 11894 & & 4 & & $6 / 25 / 07$ & 295.2019 & 301.6062 & 6.4043 & $6 / 26 / 07$ & 300.5834 & 1.0228 & 15.9705 & 3.1 \\
\hline & 11895 & & 5 & & 6/25/07 & 293.9587 & 300.2982 & 6.3395 & $6 / 26 / 07$ & 299.2253 & 1.0729 & 16.9240 & \\
\hline & 11896 & & 6 & & $6 / 25 / 07$ & 294.1719 & 300.42 & 6.2481 & $6 / 26 / 07$ & 299.3709 & 1.0491 & 16.7907 & \\
\hline
\end{tabular}




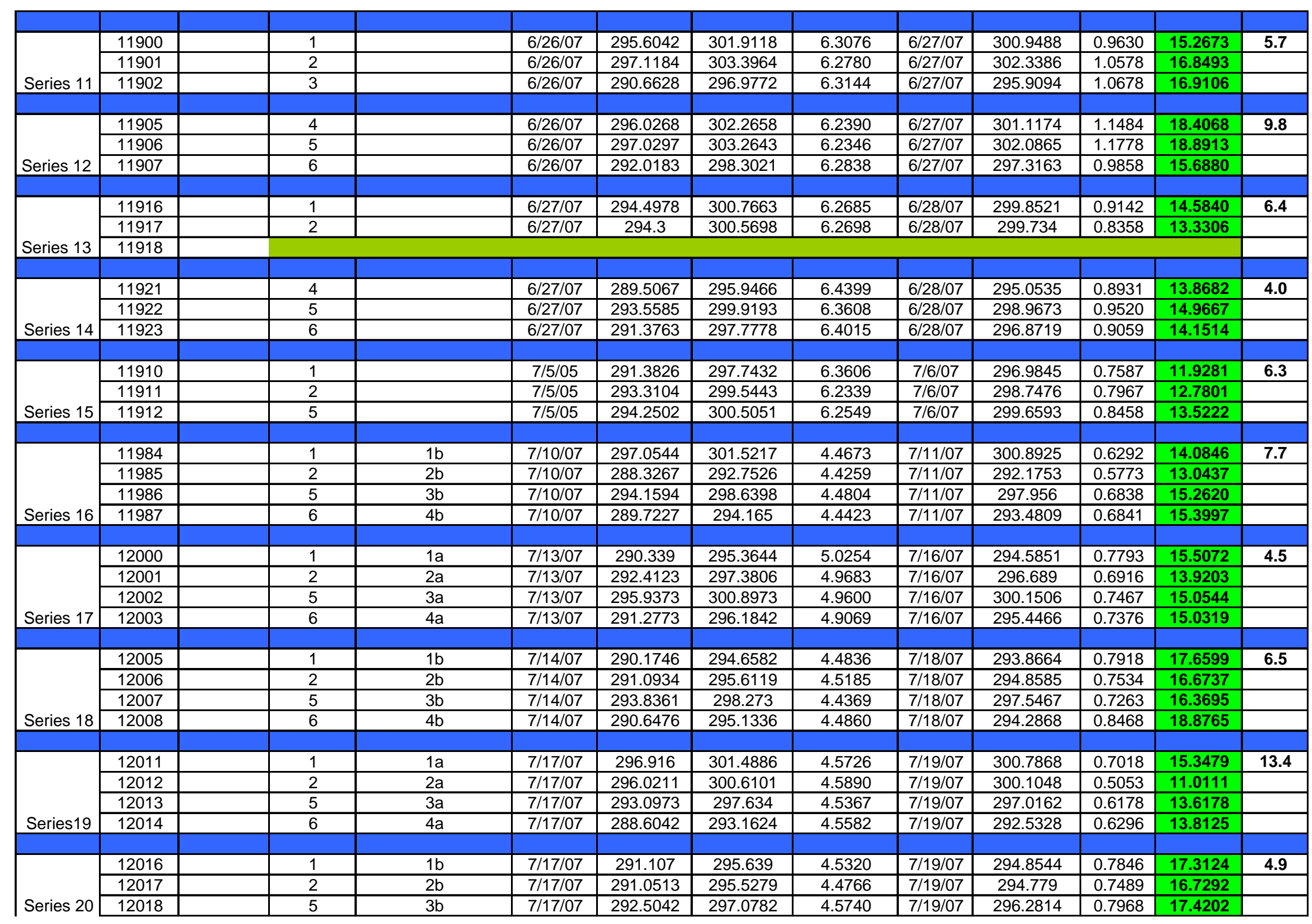




\begin{tabular}{|c|c|c|c|c|c|c|c|c|c|c|c|c|c|}
\hline \multirow{5}{*}{ Series 21} & 12019 & & 6 & $4 \mathrm{~b}$ & $7 / 17 / 07$ & 292.0998 & 296.6452 & 4.5454 & $7 / 19 / 07$ & 295.7925 & 0.8527 & 18.7596 & \\
\hline & 11945 & & 1 & $1 \mathrm{~b}$ & $7 / 19 / 07$ & 285.8336 & 290.8555 & 5.0219 & $7 / 23 / 07$ & 290.3107 & 0.5448 & 10.8485 & 6.4 \\
\hline & 11946 & & 2 & $2 \mathrm{~b}$ & $7 / 19 / 07$ & 291.5184 & 296.6293 & 5.1109 & $7 / 23 / 07$ & 296.0166 & 0.6127 & 11.9881 & \\
\hline & 11947 & & 5 & $3 b$ & $7 / 19 / 07$ & 289.8509 & 295.0326 & 5.1817 & $7 / 23 / 07$ & 294.3969 & 0.6357 & 12.2682 & \\
\hline & & & & & & & & & & & & & \\
\hline \multirow[b]{4}{*}{ Series 22} & 12143 & & 1 & $1 \mathrm{~b}$ & $7 / 24 / 07$ & 286.0909 & 291.6234 & 5.5325 & $7 / 26 / 07$ & 290.5314 & 1.0920 & 19.7379 & 2.6 \\
\hline & 12144 & & 2 & $2 b$ & $7 / 24 / 07$ & 296.2186 & 301.786 & 5.5674 & $7 / 26 / 07$ & 300.6584 & 1.1276 & 20.2536 & \\
\hline & 12145 & & 5 & $3 b$ & $7 / 24 / 07$ & 288.6546 & 294.4172 & 5.7626 & $7 / 26 / 07$ & 293.207 & 1.2102 & 21.0009 & \\
\hline & 12146 & & 6 & $4 b$ & $7 / 24 / 07$ & 294.3823 & 300.0375 & 5.6552 & $7 / 26 / 07$ & 298.8818 & 1.1557 & 20.4361 & \\
\hline & & & & & & & & & & & & & \\
\hline \multirow[b]{3}{*}{ Series 23} & 12150 & & 2 & $2 a$ & $7 / 24 / 07$ & 295.0208 & 300.6382 & 5.6174 & $7 / 26 / 07$ & 299.5983 & 1.0399 & 18.5121 & 3.4 \\
\hline & 12151 & & 3 & $3 a$ & $7 / 24 / 07$ & 283.7716 & 289.4413 & 5.6697 & $7 / 26 / 07$ & 288.4461 & 0.9952 & 17.5530 & \\
\hline & 12152 & & 4 & $4 a$ & $7 / 24 / 07$ & 286.9984 & 292.6396 & 5.6412 & $7 / 26 / 07$ & 291.658 & 0.9816 & 17.4006 & \\
\hline \multirow{10}{*}{ Series 24} & & & & & & & & & & & & & \\
\hline & 12443 & \begin{tabular}{|l|} 
E02038-03 \\
\end{tabular} & 1 & 1 140micron & $8 / 14 / 07$ & 323.7065 & 328.4221 & 4.7156 & $8 / 16 / 07$ & 327.5051 & 0.9170 & 19.4461 & 11.4 \\
\hline & 12444 & E02038-04 & 2 & 2 140micron & $8 / 14 / 07$ & 315.4444 & 320.0646 & 4.6202 & $8 / 16 / 07$ & 319.4502 & 0.6144 & 13.2981 & \\
\hline & 12445 & E02038-05 & 3 & 3 140micron & $8 / 14 / 07$ & 319.308 & 323.9439 & 4.6359 & $8 / 16 / 07$ & 323.1176 & 0.8263 & 17.8239 & \\
\hline & 12446 & E02038-06 & 4 & 4 140micron & $8 / 14 / 07$ & 312.6616 & 317.3194 & 4.6578 & $8 / 16 / 07$ & 316.5391 & 0.7803 & 16.7525 & \\
\hline & 12447 & E02038-07 & 5 & 5 140micron & $8 / 14 / 07$ & 325.5572 & 330.1698 & 4.6126 & $8 / 16 / 07$ & 329.3949 & 0.7749 & 16.7996 & \\
\hline & 12448 & E02038-08 & 6 & 6 140micron & $8 / 14 / 07$ & 307.7391 & 312.427 & 4.6879 & $8 / 16 / 07$ & 311.569 & 0.8580 & 18.3024 & \\
\hline & 12449 & E02038-09 & 1 & 1140 micron & $8 / 14 / 07$ & 322.0509 & 326.6945 & 4.6436 & $8 / 16 / 07$ & 325.7902 & 0.9043 & 19.4741 & \\
\hline & 12450 & E02038-10 & 2 & 2140 micron & $8 / 14 / 07$ & 316.9905 & 321.6914 & 4.7009 & $8 / 16 / 07$ & 320.8928 & 0.7986 & 16.9882 & \\
\hline & & & & & & & & & & & & & \\
\hline \multirow[b]{6}{*}{ Series 25} & 12453 & E02042-02 & 3 & 3 140micron & $8 / 14 / 07$ & 315.3053 & 320.1823 & 4.8770 & $8 / 16 / 07$ & 319.4638 & 0.7185 & 14.7324 & 11.9 \\
\hline & 12454 & E02042-03 & 4 & 4 140micron & $8 / 14 / 07$ & 321.8331 & 326.6925 & 4.8594 & $8 / 16 / 07$ & 325.9712 & 0.7213 & 14.8434 & \\
\hline & 12455 & \begin{tabular}{|l}
$E 02042-04$ \\
\end{tabular} & 5 & 5 140micron & $8 / 14 / 07$ & 317.1041 & 321.9614 & 4.8573 & $8 / 16 / 07$ & 321.1673 & 0.7941 & 16.3486 & \\
\hline & 12456 & \begin{tabular}{|l}
$E 02042-05$ \\
\end{tabular} & 6 & 6 140micron & $8 / 14 / 07$ & 324.2906 & 329.1146 & 4.8240 & $8 / 16 / 07$ & 328.3714 & 0.7432 & 15.4063 & \\
\hline & 12457 & E02042-06 & 1 & 1a Drilled Thimble & $8 / 15 / 07$ & 321.1469 & 325.9024 & 4.7555 & $8 / 17 / 07$ & 325.224 & 0.6784 & 14.2656 & \\
\hline & 12458 & E02042-07 & 2 & 2a Drilled Thimble & $8 / 15 / 07$ & 312.9501 & 317.7253 & 4.7752 & $8 / 17 / 07$ & 317.1867 & 0.5386 & 11.2791 & \\
\hline \multirow{7}{*}{ Series 26} & & & & & & & & & & & & & \\
\hline & 12461 & E02044-02 & 3 & 3a Drilled Thimble & $8 / 15 / 07$ & 323.4404 & 328.397 & 4.9566 & $8 / 17 / 07$ & 327.7299 & 0.6671 & 13.4588 & 11.3 \\
\hline & 12462 & E02044-03 & 4 & 4a Drilled Thimble & $8 / 15 / 07$ & 321.7311 & 326.5894 & 4.8583 & $8 / 17 / 07$ & 325.8774 & 0.7120 & 14.6553 & \\
\hline & 12463 & E02044-04 & 5 & 5a Drilled Thimble & $8 / 15 / 07$ & 317.177 & 322.0988 & 4.9218 & $8 / 17 / 07$ & 321.3399 & 0.7589 & 15.4192 & \\
\hline & 12464 & E02044-05 & 6 & 6a Drilled Thimble & $8 / 15 / 07$ & 319.6735 & 324.546 & 4.8725 & $8 / 17 / 07$ & 323.9208 & 0.6252 & 12.8312 & \\
\hline & 12466 & E02044-07 & 2 & 2b Drilled Thimble & $8 / 15 / 07$ & 319.9139 & 324.8545 & 4.9406 & $8 / 17 / 07$ & 324.0139 & 0.8406 & 17.0141 & \\
\hline & & & & & & & & & & & & & \\
\hline \multirow[b]{6}{*}{ Series 27} & 12469 & E02045-02 & 3 & 3b Drilled Thimble & $8 / 15 / 07$ & 310.8439 & 314.8804 & 4.0365 & $8 / 17 / 07$ & 314.1036 & 0.7768 & 19.2444 & 6.2 \\
\hline & 12470 & E02045-03 & 4 & 4b Drilled Thimble & $8 / 15 / 07$ & 336.0691 & 340.057 & 3.9879 & $8 / 17 / 07$ & 339.2373 & 0.8197 & 20.5547 & \\
\hline & 12471 & E02045-04 & 5 & 5b Drilled Thimble & $8 / 15 / 07$ & 319.3587 & 323.2837 & 3.9250 & $8 / 17 / 07$ & 322.4999 & 0.7838 & 19.9694 & \\
\hline & 12472 & E02045-05 & 6 & 6b Drilled Thimble & $8 / 15 / 07$ & 315.8663 & 319.9247 & 4.0584 & $8 / 17 / 07$ & 319.2183 & 0.7064 & 17.4059 & \\
\hline & 12473 & E02045-06 & 1 & 1a Drilled Thimble & $8 / 16 / 07$ & 319.954 & 323.8547 & 3.9007 & $8 / 20 / 07$ & 323.0929 & 0.7618 & 19.5298 & \\
\hline & 12474 & E02045-07 & 2 & 2a Drilled Thimble & $8 / 16 / 07$ & 316.7147 & 320.6512 & 3.9365 & $8 / 20 / 07$ & 319.8315 & 0.8197 & 20.8231 & \\
\hline & & & & & & & & & & & & & \\
\hline
\end{tabular}




\begin{tabular}{|c|c|c|c|c|c|c|c|c|c|c|c|c|c|}
\hline \multirow[b]{6}{*}{ Series 28} & 12477 & E02046-02 & 3 & 3a Drilled Thimble & $8 / 16 / 07$ & 318.2251 & 322.2307 & 4.0056 & $8 / 20 / 07$ & 321.3529 & 0.8778 & 21.9143 & 13.7 \\
\hline & 12478 & E02046-03 & 4 & 4a Drilled Thimble & $8 / 16 / 07$ & 311.9847 & 316.0836 & 4.0989 & $8 / 20 / 07$ & 315.0936 & 0.9900 & 24.1528 & \\
\hline & 12479 & E02046-04 & 5 & $5 a$ Drilled Thimble & $8 / 16 / 07$ & 317.8384 & 321.791 & 3.9526 & $8 / 20 / 07$ & 320.9816 & 0.8094 & 20.4777 & \\
\hline & 12480 & E02046-05 & 6 & 6a Drilled Thimble & $8 / 16 / 07$ & 311.8318 & 315.849 & 4.0172 & $8 / 20 / 07$ & 315.008 & 0.8410 & 20.9350 & \\
\hline & 12481 & E02046-06 & 1 & 1a Drilled Thimble & $8 / 16 / 07$ & 308.3002 & 312.338 & 4.0378 & $8 / 20 / 07$ & 311.6542 & 0.6838 & 16.9350 & \\
\hline & 12482 & E02046-07 & 2 & 2b Drilled Thimble & $8 / 16 / 07$ & 315.7201 & 319.8027 & 4.0826 & $8 / 20 / 07$ & 319.1004 & 0.7023 & 17.2023 & \\
\hline & & & & & & & & & & & & & \\
\hline \multirow[b]{4}{*}{ Series 29} & 12486 & E02047-04 & 3 & 3b Drilled Thimble & $8 / 16 / 07$ & 317.6151 & 321.7358 & 4.1207 & $8 / 20 / 07$ & 320.8491 & 0.8867 & 21.5182 & 13.2 \\
\hline & 12487 & E02047-05 & 4 & 4b Drilled Thimble & $8 / 16 / 07$ & 307.4027 & 311.4551 & 4.0524 & $8 / 20 / 07$ & 310.7606 & 0.6945 & 17.1380 & \\
\hline & 12488 & E02047-06 & 5 & 5b Drilled Thimble & $8 / 16 / 07$ & 317.5195 & 321.7166 & 4.1971 & $8 / 20 / 07$ & 320.7535 & 0.9631 & 22.9468 & \\
\hline & 12489 & E02047-07 & 6 & 6b Drilled Thimble & $8 / 16 / 07$ & 301.5519 & 305.7135 & 4.1616 & $8 / 20 / 07$ & 304.9375 & 0.7760 & 18.6467 & \\
\hline & & & & & & & & & & & & & \\
\hline \multirow{5}{*}{ Series 30} & 12492 & E02048-02 & 1 & 1a Drilled Thimble & $8 / 17 / 07$ & 316.5557 & 321.746 & 5.1903 & $8 / 22 / 07$ & 320.8543 & 0.8917 & 17.1801 & 10.1 \\
\hline & 12493 & E02048-03 & 2 & 2a Drilled Thimble & $8 / 17 / 07$ & 318.3626 & 323.5369 & 5.1743 & $8 / 22 / 07$ & 322.8035 & 0.7334 & 14.1739 & \\
\hline & 12494 & E02048-04 & 3 & 3a Drilled Thimble & $8 / 17 / 07$ & 314.362 & 319.6562 & 5.2942 & $8 / 22 / 07$ & 318.8844 & 0.7718 & 14.5782 & \\
\hline & 12495 & E02048-05 & 4 & 4a Drilled Thimble & $8 / 17 / 07$ & 316.432 & 321.5931 & 5.1611 & $8 / 22 / 07$ & 320.877 & 0.7161 & 13.8749 & \\
\hline & & & & & & & & & & & & & \\
\hline \multirow[b]{6}{*}{ Series 31} & 12499 & E02049-02 & 5 & 5a Drilled Thimble & $8 / 17 / 07$ & 314.298 & 318.2588 & 3.9608 & $8 / 22 / 07$ & 317.601 & 0.6578 & 16.6078 & 13.5 \\
\hline & 12500 & E02049-03 & 6 & 6a Drilled Thimble & $8 / 17 / 07$ & 316.522 & 320.571 & 4.0490 & $8 / 22 / 07$ & 319.7938 & 0.7772 & 19.1949 & \\
\hline & 12501 & E02049-04 & 5 & 5a Drilled Thimble & $9 / 18 / 07$ & 318.8982 & 322.8795 & 3.9813 & $9 / 21 / 07$ & 321.9143 & 0.9652 & 24.2433 & \\
\hline & 12502 & E02049-05 & 6 & 6a Drilled Thimble & $9 / 18 / 07$ & 317.9778 & 321.9882 & 4.0104 & $9 / 21 / 07$ & 321.1876 & 0.8006 & 19.9631 & \\
\hline & 12503 & E02049-06 & 1 & 1a Drilled Thimble & $9 / 19 / 07$ & 318.9217 & 322.9685 & 4.0468 & $9 / 21 / 07$ & 322.0513 & 0.9172 & 22.6648 & \\
\hline & 12504 & E02049-07 & 2 & 2a Drilled Thimble & $9 / 19 / 07$ & 306.8737 & 310.8001 & 3.9264 & $9 / 21 / 07$ & 310.0535 & 0.7466 & 19.0149 & \\
\hline & & & & & & & & & & & & & \\
\hline \multirow[b]{4}{*}{ Series 32} & 12658 & E02095-02 & 1 & 1a Drilled Thimble & $8 / 28 / 07$ & 287.5933 & 292.247 & 4.6537 & $9 / 5 / 07$ & 291.3379 & 0.9091 & 19.5350 & 10.4 \\
\hline & 12659 & E02095-03 & 2 & 2a Drilled Thimble & $8 / 28 / 07$ & 287.7254 & 292.3184 & 4.5930 & $9 / 5 / 07$ & 291.5622 & 0.7562 & 16.4642 & \\
\hline & 12660 & E02095-04 & 3 & 3a Drilled Thimble & $8 / 28 / 07$ & 289.4624 & 294.0404 & 4.5780 & $9 / 5 / 07$ & 293.3203 & 0.7201 & 15.7296 & \\
\hline & 12661 & E02095-05 & 4 & 4a Drilled Thimble & $8 / 28 / 07$ & 287.8841 & 292.4592 & 4.5751 & $9 / 5 / 07$ & 291.5964 & 0.8628 & 18.8586 & \\
\hline \multirow[b]{10}{*}{ Series 33} & & & & & & & & & & & & & \\
\hline & 12637 & E02093-02 & 1 & 1a Drilled Thimble & 9/18/07 & 304.2039 & 308.8405 & 4.6366 & 9/21/07 & 307.7238 & 1.1167 & 24.0845 & 7.3 \\
\hline & 12638 & E02093-03 & 2 & 2a Drilled Thimble & $9 / 18 / 07$ & 305.3055 & 309.9623 & 4.6568 & $9 / 21 / 07$ & 308.8115 & 1.1508 & 24.7122 & \\
\hline & 12639 & E02093-04 & 3 & 3a Drilled Thimble & $9 / 18 / 07$ & 304.3309 & 308.9436 & 4.6127 & $9 / 21 / 07$ & 307.9283 & 1.0153 & 22.0110 & \\
\hline & 12640 & E02093-05 & 4 & 4a Drilled Thimble & $9 / 18 / 07$ & 310.7514 & 315.3872 & 4.6358 & $9 / 21 / 07$ & 314.4021 & 0.9851 & 21.2498 & \\
\hline & 12651 & E02093-06 & 5 & 5a Drilled Thimble & $8 / 28 / 07$ & 286.33 & 290.9677 & 4.6377 & $9 / 5 / 07$ & 289.94 & 1.0277 & 22.1597 & \\
\hline & 12652 & E02093-07 & 6 & 6a Drilled Thimble & $8 / 28 / 07$ & 289.3173 & 293.887 & 4.5697 & $9 / 5 / 07$ & 292.9581 & 0.9289 & 20.3274 & \\
\hline & 12653 & E02093-08 & 1 & 1a Drilled Thimble & $8 / 29 / 07$ & 297.4142 & 302.0407 & 4.6265 & $9 / 5 / 07$ & 301.082 & 0.9587 & 20.7219 & \\
\hline & 12654 & E02093-09 & 2 & 2a Drilled Thimble & $8 / 29 / 07$ & 293.1863 & 297.8278 & 4.6415 & $9 / 5 / 07$ & 296.7193 & 1.1085 & 23.8824 & \\
\hline & 12655 & E02093-10 & 3 & 3a Drilled Thimble & $8 / 29 / 07$ & 296.1488 & 300.8328 & 4.6840 & $9 / 5 / 07$ & 299.8577 & 0.9751 & 20.8177 & \\
\hline \multirow[b]{6}{*}{ Series 34} & & & & & & & & & & & & & \\
\hline & 12507 & E02050-02 & 3 & 3a Drilled Thimble & $9 / 19 / 07$ & 318.1582 & 322.2144 & 4.0562 & $9 / 21 / 07$ & 321.4251 & 0.7893 & 19.4591 & 4.8 \\
\hline & 12508 & E02050-03 & 4 & 4a Drilled Thimble & $9 / 19 / 07$ & 315.4461 & 319.4905 & 4.0444 & $9 / 21 / 07$ & 318.7 & 0.7905 & 19.5455 & \\
\hline & 12509 & E02050-04 & 5 & 5a Drilled Thimble & $9 / 19 / 07$ & 313.5171 & 317.5971 & 4.0800 & $9 / 21 / 07$ & 316.7539 & 0.8432 & 20.6667 & \\
\hline & 12511 & E02050-06 & 6 & 6a Drilled Thimble & $9 / 19 / 07$ & 316.676 & 320.7299 & 4.0539 & $9 / 21 / 07$ & 319.8586 & 0.8713 & 21.4929 & \\
\hline & 12512 & E02050-07 & 1 & 1a Drilled Thimble & $9 / 24 / 07$ & 322.2567 & 326.1808 & 3.9241 & $9 / 27 / 07$ & 325.1695 & 1.0113 & 25.7715 & \\
\hline
\end{tabular}




\begin{tabular}{|c|c|c|c|c|c|c|c|c|c|c|c|c|c|}
\hline & & & & & & & & & & & & & \\
\hline \multirow[b]{6}{*}{ Series 35} & 12514 & E02052-02 & 1 & 1a Drilled Thimble & $9 / 20 / 07$ & 317.4239 & 322.0377 & 4.6138 & 9/21/07 & 321.1682 & 0.8695 & 18.8456 & 11.3 \\
\hline & 12515 & E02052-03 & 2 & 2a Drilled Thimble & $9 / 20 / 07$ & 316.7504 & 321.3155 & 4.5651 & $9 / 21 / 07$ & 320.2105 & 1.1050 & 24.2054 & \\
\hline & 12516 & E02052-04 & 3 & 3a Drilled Thimble & $9 / 20 / 07$ & 311.4542 & 316.0619 & 4.6077 & $9 / 21 / 07$ & 315.2202 & 0.8417 & 18.2672 & \\
\hline & 12517 & E02052-05 & 4 & 4a Drilled Thimble & $9 / 20 / 07$ & 311.3394 & 315.95 & 4.6106 & $9 / 21 / 07$ & 315.0917 & 0.8583 & 18.6158 & \\
\hline & 12518 & E02052-06 & 5 & 5a Drilled Thimble & $9 / 20 / 07$ & 306.0085 & 310.703 & 4.6945 & $9 / 21 / 07$ & 309.7476 & 0.9554 & 20.3515 & \\
\hline & 12519 & E02052-07 & 6 & 6a Drilled Thimble & $9 / 20 / 07$ & 317.219 & 321.9793 & 4.7603 & $9 / 21 / 07$ & 321.0773 & 0.9020 & 18.9484 & \\
\hline & & & & & & & & & & & & & \\
\hline \multirow[b]{6}{*}{ Series 36} & 12522 & E02053-02 & 1 & 1a Drilled Thimble & 9/21/07 & 316.058 & 320.7603 & 4.7023 & $9 / 27 / 07$ & 319.9775 & 0.7828 & 16.6472 & 2.6 \\
\hline & 12523 & E02053-03 & 2 & 2a Drilled Thimble & $9 / 21 / 07$ & 317.0631 & 321.7381 & 4.6750 & $9 / 27 / 07$ & 320.923 & 0.8151 & 17.4353 & \\
\hline & 12524 & E02053-04 & 3 & 3a Drilled Thimble & $9 / 21 / 07$ & 304.8478 & 309.5295 & 4.6817 & $9 / 27 / 07$ & 308.7464 & 0.7831 & 16.7268 & \\
\hline & 12525 & E02053-05 & 4 & 4a Drilled Thimble & $9 / 21 / 07$ & 319.4941 & 324.1499 & 4.6558 & $9 / 27 / 07$ & 323.3609 & 0.7890 & 16.9466 & \\
\hline & 12526 & E02053-06 & 5 & 5a Drilled Thimble & $9 / 21 / 07$ & 319.412 & 324.1114 & 4.6994 & $9 / 27 / 07$ & 323.2791 & 0.8323 & 17.7108 & \\
\hline & 12527 & E02053-07 & 6 & 6a Drilled Thimble & $9 / 21 / 07$ & 315.6156 & 320.3762 & 4.7606 & $9 / 27 / 07$ & 319.542 & 0.8342 & 17.5230 & \\
\hline \multirow[b]{5}{*}{ Series 37} & & & & & & & & & & & & & \\
\hline & 12529 & E02054-02 & 2 & 2a Drilled Thimble & $9 / 24 / 07$ & 311.738 & 316.4992 & 4.7612 & $9 / 27 / 07$ & 315.4968 & 1.0024 & 21.0535 & 13.7 \\
\hline & 12530 & E02054-03 & 3 & 3a Drilled Thimble & $9 / 24 / 07$ & 321.8186 & 326.3623 & 4.5437 & $9 / 27 / 07$ & 325.4512 & 0.9111 & 20.0519 & \\
\hline & 12531 & E02054-04 & 4 & 4a Drilled Thimble & 9/24/07 & 307.3071 & 311.7855 & 4.4784 & 9/27/07 & 311.0002 & 0.7853 & 17.5353 & \\
\hline & 12532 & E02054-05 & 5 & 5a Drilled Thimble & $9 / 24 / 07$ & 313.5352 & 318.1776 & 4.6424 & $9 / 27 / 07$ & 317.0458 & 1.1318 & 24.3796 & \\
\hline & & & & & & & & & & & & & \\
\hline \multirow[b]{4}{*}{ Series 38} & 12535 & E02055-02 & 6 & 6a Drilled Thimble & 9/24/07 & 325.3529 & 329.8714 & 4.5185 & 9/27/07 & 328.982 & 0.8894 & 19.6835 & 10.3 \\
\hline & 12536 & E02055-03 & 1 & 1a Drilled Thimble & $9 / 25 / 07$ & 313.3728 & 317.9732 & 4.6004 & $9 / 27 / 07$ & 316.8903 & 1.0829 & 23.5393 & \\
\hline & 12537 & E02055-04 & 2 & 2a Drilled Thimble & $9 / 25 / 07$ & 317.0306 & 321.6274 & 4.5968 & $9 / 27 / 07$ & 320.5892 & 1.0382 & 22.5853 & \\
\hline & 12538 & E02055-05 & 3 & 3a Drilled Thimble & $9 / 25 / 07$ & 316.281 & 320.858 & 4.5770 & $9 / 27 / 07$ & 319.9857 & 0.8723 & 19.0583 & \\
\hline & & & & & & & & & & & & & \\
\hline \multirow[b]{3}{*}{ Series 39} & 12540 & E02056-02 & 4 & 4a Drilled Thimble & $9 / 25 / 07$ & 314.4915 & 319.0784 & 4.5869 & $9 / 27 / 07$ & 318.2498 & 0.8286 & 18.0645 & 8.5 \\
\hline & 12562 & E02056-04 & 5 & 5a Drilled Thimble & $9 / 25 / 07$ & 302.3836 & 307.1183 & 4.7347 & $9 / 27 / 07$ & 306.1165 & 1.0018 & 21.1587 & \\
\hline & 12563 & E02056-06 & 6 & 6a Drilled Thimble & $9 / 25 / 07$ & 308.2408 & 312.9086 & 4.6678 & $9 / 27 / 07$ & 311.9365 & 0.9721 & 20.8257 & \\
\hline & & & & & & & & & & & & & \\
\hline \multirow{7}{*}{ Series 40} & 13103 & E02187-02 & 1 & 1a Drilled Thimble & $10 / 30 / 07$ & 297.5969 & 300.9266 & 3.3297 & $11 / 2 / 07$ & 300.1487 & 0.7779 & 23.3625 & 9.6 \\
\hline & 13104 & E02187-03 & 2 & 2a Drilled Thimble & $10 / 30 / 07$ & 296.8633 & 300.13 & 3.2667 & $11 / 2 / 07$ & 299.2089 & 0.9211 & 28.1967 & \\
\hline & 13105 & E02187-04 & 3 & 3a Drilled Thimble & $10 / 30 / 07$ & 305.0563 & 308.4696 & 3.4133 & $11 / 2 / 07$ & 307.623 & 0.8466 & 24.8030 & \\
\hline & 13106 & E02187-05 & 4 & 4a Drilled Thimble & $10 / 30 / 07$ & 299.363 & 302.6736 & 3.3106 & $11 / 2 / 07$ & 301.7622 & 0.9114 & 27.5298 & \\
\hline & 13107 & E02187-06 & 5 & 5a Drilled Thimble & $10 / 30 / 07$ & 294.2738 & 297.4628 & 3.1890 & $11 / 2 / 07$ & 296.4861 & 0.9767 & 30.6272 & \\
\hline & 13108 & E02187-07 & 6 & 6a Drilled Thimble & $10 / 30 / 07$ & 298.8103 & 302.0374 & 3.2271 & $11 / 2 / 07$ & 301.1792 & 0.8582 & 26.5935 & \\
\hline & & & & & & & & & & & & & \\
\hline \multirow{7}{*}{ Series 41} & 13117 & E02189-02 & 1 & 1a Drilled Thimble & $10 / 31 / 07$ & 275.3932 & 278.1845 & 2.7913 & $11 / 2 / 07$ & 277.5956 & 0.5889 & 21.0977 & 6.8 \\
\hline & 13112 & E02189-03 & 2 & 2a Drilled Thimble & $10 / 31 / 07$ & 298.4955 & 301.3237 & 2.8282 & $11 / 2 / 07$ & 300.6776 & 0.6461 & 22.8449 & \\
\hline & 13113 & E02189-04 & 3 & 3a Drilled Thimble & $10 / 31 / 07$ & 304.1104 & 306.9545 & 2.8441 & $11 / 2 / 07$ & 306.2159 & 0.7386 & 25.9696 & \\
\hline & 13114 & E02189-05 & 4 & 4a Drilled Thimble & $10 / 31 / 07$ & 299.8973 & 302.7381 & 2.8408 & $11 / 2 / 07$ & 302.0651 & 0.6730 & 23.6905 & \\
\hline & 13115 & E02189-06 & 5 & 5a Drilled Thimble & $10 / 31 / 07$ & 301.2121 & 304.08 & 2.8679 & $11 / 2 / 07$ & 303.4174 & 0.6626 & 23.1040 & \\
\hline & 13116 & E02189-07 & 6 & 6a Drilled Thimble & $10 / 31 / 07$ & 277.6 & 280.4293 & 2.8293 & $11 / 2 / 07$ & 279.757 & 0.6723 & 23.7621 & \\
\hline & & & & & & & & & & & & & \\
\hline
\end{tabular}




\begin{tabular}{|c|c|c|c|c|c|c|c|c|c|c|c|c|c|}
\hline \multirow[b]{8}{*}{ Series 42} & 13119 & E02191-02 & 1 & 1a Drilled Thimble & $11 / 1 / 07$ & 302.8403 & 305.885 & 3.0447 & $11 / 5 / 07$ & 305.0321 & 0.8529 & 28.0126 & 6.3 \\
\hline & 13120 & E02191-03 & 2 & 2a Drilled Thimble & $11 / 1 / 07$ & 306.2385 & 309.3156 & 3.0771 & $11 / 5 / 07$ & 308.5081 & 0.8075 & 26.2422 & \\
\hline & 13121 & E02191-04 & 3 & 3a Drilled Thimble & $11 / 1 / 07$ & 301.7282 & 304.7785 & 3.0503 & $11 / 5 / 07$ & 304.0277 & 0.7508 & 24.6140 & \\
\hline & 13122 & E02191-05 & 4 & 4a Drilled Thimble & $11 / 1 / 07$ & 306.1713 & 309.2107 & 3.0394 & $11 / 5 / 07$ & 308.4529 & 0.7578 & 24.9326 & \\
\hline & 13123 & E02191-06 & 5 & $5 a$ Drilled Thimble & $11 / 1 / 07$ & 300.6467 & 303.6224 & 2.9757 & $11 / 5 / 07$ & 302.7907 & 0.8317 & 27.9497 & \\
\hline & 13124 & E02191-07 & 6 & 6a Drilled Thimble & $11 / 1 / 07$ & 298.9502 & 301.8389 & 2.8887 & $11 / 5 / 07$ & 301.0691 & 0.7698 & 26.6487 & \\
\hline & 13125 & E02191-08 & 1 & 1a Drilled Thimble & $11 / 5 / 07$ & 298.4658 & 301.3624 & 2.8966 & $11 / 6 / 07$ & 300.5343 & 0.8281 & 28.5887 & \\
\hline & 13126 & E02191-09 & 2 & 2a Drilled Thimble & $11 / 5 / 07$ & 303.3735 & 306.1924 & 2.8189 & $11 / 6 / 07$ & 305.3684 & 0.8240 & 29.2313 & \\
\hline & & & & & & & & & & & & & \\
\hline \multirow[b]{6}{*}{ Series 43} & 13131 & E02192-04 & 3 & 3a Drilled Thimble & $11 / 5 / 07$ & 309.474 & 312.271 & 2.797 & $11 / 6 / 07$ & 311.3849 & 0.8861 & 31.6804 & 7.5 \\
\hline & 13132 & E02192-05 & 4 & 4a Drilled Thimble & $11 / 5 / 07$ & 306.8462 & 309.6772 & 2.831 & $11 / 6 / 07$ & 308.9399 & 0.7373 & 26.0438 & \\
\hline & 13133 & E02192-06 & 5 & 5a Drilled Thimble & $11 / 5 / 07$ & 310.6059 & 313.4004 & 2.7945 & $11 / 6 / 07$ & 312.6199 & 0.7805 & 27.9299 & \\
\hline & 13134 & E02192-07 & 6 & 6a Drilled Thimble & $11 / 5 / 07$ & 308.6263 & 311.5126 & 2.8863 & $11 / 6 / 07$ & 310.6812 & 0.8314 & 28.8050 & \\
\hline & 13135 & E02192-08 & 1 & $1 \mathrm{~b}$ Drilled Thimble & $11 / 6 / 07$ & 299.8368 & 302.6149 & 2.7781 & $11 / 7 / 07$ & 301.7336 & 0.8813 & 31.7231 & \\
\hline & 13136 & E02192-09 & 2 & 2b Drilled Thimble & $11 / 6 / 07$ & 304.2979 & 307.0843 & 2.7864 & $11 / 7 / 07$ & 306.2631 & 0.8212 & 29.4717 & \\
\hline & & & & & & & & & & & & & \\
\hline \multirow[b]{6}{*}{ Series 44} & 13139 & E02193-02 & 1 & 1a Drilled Thimble & $11 / 6 / 07$ & 306.0854 & 308.8435 & 2.7581 & $11 / 7 / 07$ & 308.0726 & 0.7709 & 27.9504 & 9.2 \\
\hline & 13140 & E02193-03 & 2 & 2a Drilled Thimble & $11 / 6 / 07$ & 300.0987 & 302.8911 & 2.7924 & $11 / 7 / 07$ & 302.0424 & 0.8487 & 30.3932 & \\
\hline & 13141 & E02193-04 & 3 & 3a Drilled Thimble & $11 / 6 / 07$ & 302.8864 & 305.6156 & 2.7292 & $11 / 7 / 07$ & 304.8073 & 0.8083 & 29.6167 & \\
\hline & 13142 & E02193-05 & 4 & 4a Drilled Thimble & $11 / 6 / 07$ & 310.902 & 313.6503 & 2.7483 & $11 / 7 / 07$ & 312.7393 & 0.9110 & 33.1478 & \\
\hline & 13143 & E02193-06 & 5 & 5a Drilled Thimble & $11 / 6 / 07$ & 300.3086 & 303.055 & 2.7464 & $11 / 7 / 07$ & 302.1514 & 0.9036 & 32.9013 & \\
\hline & 13144 & E02193-07 & 6 & 6a Drilled Thimble & $11 / 6 / 07$ & 316.8667 & 319.7195 & 2.8528 & $11 / 7 / 07$ & 318.9742 & 0.7453 & 26.1252 & \\
\hline & & & & & & & & & & & & & \\
\hline \multirow{5}{*}{ Series 45} & 13146 & E02194-02 & 1 & 1b Drilled Thimble & $11 / 7 / 07$ & 310.7381 & 313.4921 & 2.754 & $11 / 13 / 07$ & 312.6601 & 0.8320 & 30.2106 & 6.6 \\
\hline & 13147 & E02194-03 & 2 & 2b Drilled Thimble & $11 / 7 / 07$ & 304.6489 & 307.4639 & 2.815 & $11 / 13 / 07$ & 306.6793 & 0.7846 & 27.8721 & \\
\hline & 13148 & E02194-04 & 3 & 3b Drilled Thimble & $11 / 7 / 07$ & 309.6293 & 312.4917 & 2.8624 & $11 / 13 / 07$ & 311.6315 & 0.8602 & 30.0517 & \\
\hline & 13149 & E02194-05 & 4 & 4b Drilled Thimble & $11 / 7 / 07$ & 302.1858 & 305.0288 & 2.843 & $11 / 13 / 07$ & 304.2825 & 0.7463 & 26.2504 & \\
\hline & & & & & & & & & & & & & \\
\hline \multirow[b]{6}{*}{ Series 46} & 13159 & E02195-02 & 1 & 1b Drilled Thimble & $11 / 9 / 07$ & 314.3018 & 317.3247 & 3.0229 & $11 / 13 / 07$ & 316.2507 & 1.0740 & 35.5288 & 11.8 \\
\hline & 13160 & E02195-03 & 2 & 2b Drilled Thimble & $11 / 9 / 07$ & 305.6471 & 308.6162 & 2.9691 & $11 / 13 / 07$ & 307.6334 & 0.9828 & 33.1009 & \\
\hline & 13161 & E02195-04 & 3 & 3b Drilled Thimble & $11 / 9 / 07$ & 304.9535 & 308.0921 & 3.1386 & $11 / 13 / 07$ & 307.2606 & 0.8315 & 26.4927 & \\
\hline & 13162 & E02195-05 & 4 & 4b Drilled Thimble & $11 / 9 / 07$ & 308.3881 & 311.4367 & 3.0486 & $11 / 13 / 07$ & 310.5884 & 0.8483 & 27.8259 & \\
\hline & 13163 & E02195-06 & 5 & 5b Drilled Thimble & $11 / 9 / 07$ & 303.0613 & 306.0052 & 2.9439 & $11 / 13 / 07$ & 305.0677 & 0.9375 & 31.8455 & \\
\hline & 13164 & E02195-07 & 6 & 6b Drilled Thimble & $11 / 9 / 07$ & 309.4879 & 312.4302 & 2.9423 & $11 / 13 / 07$ & 311.3976 & 1.0326 & 35.0950 & \\
\hline & & & & & & & & & & & & & \\
\hline \multirow{7}{*}{ Series 47} & 13167 & E02197-02 & 1 & 1a Drilled Thimble & $11 / 12 / 07$ & 309.131 & 312.0825 & 2.9515 & $11 / 15 / 07$ & 311.2207 & 0.8618 & 29.1987 & 8.2 \\
\hline & 13168 & E02197-03 & 2 & 2a Drilled Thimble & $11 / 12 / 07$ & 305.5208 & 308.4593 & 2.9385 & $11 / 15 / 07$ & 307.6308 & 0.8285 & 28.1947 & \\
\hline & 13169 & E02197-04 & 3 & 3a Drilled Thimble & $11 / 12 / 07$ & 307.0956 & 310.0136 & 2.918 & $11 / 15 / 07$ & 309.1406 & 0.8730 & 29.9178 & \\
\hline & 13170 & E02197-05 & 4 & 4a Drilled Thimble & $11 / 12 / 07$ & 309.6928 & 312.7016 & 3.0088 & $11 / 15 / 07$ & 311.748 & 0.9536 & 31.6937 & \\
\hline & 13171 & E02197-06 & 5 & $5 a$ Drilled Thimble & $11 / 12 / 07$ & 302.6121 & 305.5609 & 2.9488 & $11 / 15 / 07$ & 304.8353 & 0.7256 & 24.6066 & \\
\hline & 13172 & E02197-07 & 6 & 6a Drilled Thimble & $11 / 12 / 07$ & 308.643 & 311.592 & 2.949 & $11 / 15 / 07$ & 310.7502 & 0.8418 & 28.5453 & \\
\hline & & & & & & & & & & & & & \\
\hline
\end{tabular}




\begin{tabular}{|c|c|c|c|c|c|c|c|c|c|c|c|c|c|}
\hline \multirow[b]{6}{*}{ Series 48} & 13212 & E02199-02 & 1 & 1a Drilled Thimble & $11 / 14 / 07$ & 278.6357 & 281.509 & 2.8733 & $11 / 19 / 07$ & 280.7467 & 0.7623 & 26.5305 & 6.0 \\
\hline & 13213 & E02199-03 & 2 & 2a Drilled Thimble & $11 / 14 / 07$ & 288.0448 & 290.8826 & 2.8378 & \begin{tabular}{|l|}
$11 / 19 / 07$ \\
\end{tabular} & 290.1271 & 0.7555 & 26.6227 & \\
\hline & 13214 & E02199-04 & 3 & 3a Drilled Thimble & $11 / 14 / 07$ & 278.8664 & 281.699 & 2.8326 & $11 / 19 / 07$ & 280.9585 & 0.7405 & 26.1421 & \\
\hline & 13215 & E02199-05 & 4 & 4a Drilled Thimble & $11 / 14 / 07$ & 284.8412 & 287.61 & 2.7688 & $11 / 19 / 07$ & 286.8639 & 0.7461 & 26.9467 & \\
\hline & 13216 & E02199-06 & 5 & 5a Drilled Thimble & $11 / 14 / 07$ & 279.9265 & 282.6997 & 2.7732 & $11 / 19 / 07$ & 281.9171 & 0.7826 & 28.2201 & \\
\hline & 13217 & E02199-07 & 6 & 6a Drilled Thimble & $11 / 14 / 07$ & 287.7202 & 290.5701 & 2.8499 & $11 / 19 / 07$ & 289.9013 & 0.6688 & 23.4675 & \\
\hline & & & & & & & & & & & & & \\
\hline \multirow[b]{6}{*}{ Series 49} & 13219 & E02120-02 & 1 & 1b Drilled Thimble & $11 / 15 / 07$ & 287.9825 & 290.9049 & 2.9224 & $11 / 19 / 07$ & 290.2076 & 0.6973 & 23.8605 & 9.2 \\
\hline & 13220 & E02120-03 & 2 & 2b Drilled Thimble & $11 / 15 / 07$ & 277.1737 & 280.1377 & 2.964 & $11 / 19 / 07$ & 279.3392 & 0.7985 & 26.9399 & \\
\hline & 13221 & E02120-04 & 3 & 3b Drilled Thimble & $11 / 15 / 07$ & 280.3548 & 283.243 & 2.8882 & 11/19/07 & 282.4965 & 0.7465 & 25.8465 & \\
\hline & 13222 & E02120-05 & 4 & 4b Drilled Thimble & $11 / 15 / 07$ & 287.0705 & 290.04 & 2.9695 & 11/19/07 & 289.1064 & 0.9336 & 31.4396 & \\
\hline & 13223 & E02120-06 & 5 & 5b Drilled Thimble & $11 / 15 / 07$ & 286.8324 & 289.7529 & 2.9205 & 11/19/07 & 288.9382 & 0.8147 & 27.8959 & \\
\hline & 13224 & E02120-07 & 6 & 6b Drilled Thimble & $11 / 15 / 07$ & 279.4377 & 282.2783 & 2.8406 & $11 / 19 / 07$ & 281.487 & 0.7913 & 27.8568 & \\
\hline & & & & & & & & & & & & & \\
\hline \multirow[b]{5}{*}{ Series 50} & 13483 & E02252-02 & 1 & 1a Drilled Thimble & $12 / 12 / 07$ & 296.0094 & 301.1985 & 5.1891 & $12 / 13 / 07$ & 300.1966 & 1.0019 & 19.3078 & 4.5 \\
\hline & 13484 & E02252-03 & 2 & 2a Drilled Thimble & $12 / 12 / 07$ & 296.5815 & 301.6818 & 5.1003 & $12 / 13 / 07$ & 300.6735 & 1.0083 & 19.7694 & \\
\hline & 13485 & E02252-04 & 3 & 3a Drilled Thimble & $12 / 12 / 07$ & 294.2146 & 299.2074 & 4.9928 & $12 / 13 / 07$ & 298.2918 & 0.9156 & 18.3384 & \\
\hline & 13486 & E02252-05 & 4 & 4a Drilled Thimble & $12 / 12 / 07$ & 296.2525 & 301.3893 & 5.1368 & $12 / 13 / 07$ & 300.432 & 0.9573 & 18.6361 & \\
\hline & 13487 & E02252-06 & 5 & 5a Drilled Thimble & $12 / 12 / 07$ & 300.7167 & 305.8312 & 5.1145 & $12 / 13 / 07$ & 304.9303 & 0.9009 & 17.6146 & \\
\hline & & & & & & & & & & & & & \\
\hline \multirow[b]{4}{*}{ Series 51} & 13498 & E02254-02 & 1 & 1b Drilled Thimble & $12 / 13 / 07$ & 296.7337 & 301.5396 & 4.8059 & $12 / 14 / 07$ & 300.5905 & 0.9491 & 19.7486 & 4.6 \\
\hline & 13499 & E02254-03 & 2 & 2b Drilled Thimble & $12 / 13 / 07$ & 290.3686 & 295.1114 & 4.7428 & 12/14/07 & 294.1483 & 0.9631 & 20.3066 & \\
\hline & 13500 & E02254-04 & 3 & 3b Drilled Thimble & $12 / 13 / 07$ & 284.9383 & 289.7199 & 4.7816 & $12 / 14 / 07$ & 288.7081 & 1.0118 & 21.1603 & \\
\hline & 13501 & E02254-05 & 4 & 4b Drilled Thimble & $12 / 13 / 07$ & 302.1627 & 306.944 & 4.7813 & $12 / 14 / 07$ & 306.0372 & 0.9068 & 18.9656 & \\
\hline \multirow[b]{7}{*}{ Series 52} & & & & & & & & & & & & & \\
\hline & 13491 & E02253-02 & 1 & 1a Drilled Thimble & $12 / 13 / 07$ & 284.3233 & 289.1126 & 4.7893 & $12 / 14 / 07$ & 288.2708 & 0.8418 & 17.5767 & 4.7 \\
\hline & 13492 & E02253-03 & 2 & 2a Drilled Thimble & $12 / 13 / 07$ & 286.8362 & 291.5619 & 4.7257 & $12 / 14 / 07$ & 290.6655 & 0.8964 & 18.9686 & \\
\hline & 13493 & E02253-04 & 3 & 3a Drilled Thimble & $12 / 13 / 07$ & 289.2428 & 294.0337 & 4.7909 & $12 / 14 / 07$ & 293.0691 & 0.9646 & 20.1340 & \\
\hline & 13494 & E02253-05 & 4 & 4a Drilled Thimble & $12 / 13 / 07$ & 283.7612 & 288.4946 & 4.7334 & $12 / 14 / 07$ & 287.5632 & 0.9314 & 19.6772 & \\
\hline & 13495 & E02253-06 & 5 & 5a Drilled Thimble & $12 / 13 / 07$ & 289.4803 & 294.2359 & 4.7556 & 12/14/07 & 293.3057 & 0.9302 & 19.5601 & \\
\hline & 13496 & E02253-07 & 6 & 6a Drilled Thimble & $12 / 13 / 07$ & 300.0058 & 304.7017 & 4.6959 & 12/14/07 & 303.8186 & 0.8831 & 18.8058 & \\
\hline
\end{tabular}




\section{Appendix F}

\begin{tabular}{|c|c|c|c|c|c|}
\hline & Index & FilterID & DateTime & Weight & $\begin{array}{l}\text { Weighing } \\
\text { Number }\end{array}$ \\
\hline CAL4-1 & 26632 & CAL4 & 7/2/08 13:16 & 199.9957 & 1 \\
\hline CAL4-2 & 26635 & CAL4 & 7/2/08 13:18 & 199.9926 & 2 \\
\hline CAL4-3 & 26746 & CAL4 & 7/3/08 9:17 & 199.9933 & 3 \\
\hline CAL4-4 & 26956 & CAL4 & 7/7/08 8:51 & 199.9933 & 4 \\
\hline CAL4-5 & 27061 & CAL4 & 7/7/08 16:32 & 199.9938 & 5 \\
\hline CAL4-6 & 27125 & CAL4 & 7/8/08 9:28 & 199.9933 & 6 \\
\hline CAL4-7 & 27343 & CAL4 & 7/9/08 9:37 & 199.994 & 7 \\
\hline CAL4-8 & 27346 & CAL4 & 7/9/08 9:39 & 199.9923 & 8 \\
\hline CAL4-9 & 27460 & CAL4 & $7 / 10 / 08$ 12:36 & 199.9929 & 9 \\
\hline CAL4-10 & 27464 & CAL4 & $7 / 10 / 08$ 12:43 & 199.9935 & 10 \\
\hline CAL4-11 & 27584 & CAL4 & 7/11/08 9:30 & 199.9929 & 11 \\
\hline CAL4-12 & 27760 & CAL4 & $7 / 12 / 08$ 12:56 & 199.993 & 12 \\
\hline CAL4-13 & 27793 & CAL4 & $7 / 13 / 08$ 14:55 & 199.993 & 13 \\
\hline CAL4-14 & 27828 & CAL4 & $7 / 14 / 08$ 12:06 & 199.9931 & 14 \\
\hline CAL4-15 & 27977 & CAL4 & $7 / 15 / 08$ 13:21 & 199.9933 & 15 \\
\hline CAL4-16 & 28106 & CAL4 & $7 / 17 / 0814: 33$ & 199.993 & 16 \\
\hline CAL4-17 & 28483 & CAL4 & $7 / 22 / 08$ 8:25 & 199.9934 & 17 \\
\hline CAL4-18 & 28563 & CAL4 & $7 / 23 / 08$ 11:25 & 199.9928 & 18 \\
\hline CAL4-19 & 28637 & CAL4 & $7 / 24 / 08$ 12:12 & 199.9935 & 19 \\
\hline CAL4-20 & 28711 & CAL4 & $7 / 25 / 08$ 13:01 & 199.9934 & 20 \\
\hline CAL4-21 & 28899 & CAL4 & $7 / 26 / 08$ 14:53 & 199.9933 & 21 \\
\hline CAL4-22 & 28964 & CAL4 & $7 / 27 / 08$ 14:10 & 199.9934 & 22 \\
\hline CAL4-23 & 28967 & CAL4 & $7 / 27 / 08$ 14:12 & 199.9929 & 23 \\
\hline CAL4-24 & 29021 & CAL4 & $7 / 28 / 08$ 18:10 & 199.9938 & 24 \\
\hline CAL4-25 & 29066 & CAL4 & 7/29/08 7:56 & 199.9934 & 25 \\
\hline CAL4-26 & 29134 & CAL4 & $7 / 30 / 087: 21$ & 199.9944 & 26 \\
\hline CAL4-27 & 29280 & CAL4 & $7 / 31 / 08$ 15:20 & 199.9947 & 27 \\
\hline CAL4-28 & 29306 & CAL4 & $8 / 1 / 08$ 13:37 & 199.9938 & 28 \\
\hline CAL4-29 & 29368 & CAL4 & $8 / 2 / 0814: 19$ & 199.9934 & 29 \\
\hline CAL4-30 & 29413 & CAL4 & $8 / 3 / 08$ 12:26 & 199.9926 & 30 \\
\hline CAL4-31 & 29459 & CAL4 & $8 / 4 / 08$ 12:51 & 199.9936 & 31 \\
\hline CAL4-32 & 29506 & CAL4 & $8 / 5 / 08$ 12:55 & 199.9938 & 32 \\
\hline CAL4-33 & 30007 & CAL4 & $8 / 21 / 08$ 9:42 & 199.9951 & 33 \\
\hline CAL4-34 & 30262 & CAL4 & 8/28/08 9:19 & 199.9938 & 34 \\
\hline CAL4-35 & 30483 & CAL4 & 9/3/08 14:22 & 199.9934 & 35 \\
\hline WVE13832-1 & 28332 & WVE13832 & $7 / 18 / 08$ 16:43 & 281.3739 & 1 \\
\hline WVE13832-2 & 28464 & WVE13832 & $7 / 21 / 08$ 12:25 & 281.3683 & 2 \\
\hline WVE13832-3 & 28546 & WVE13832 & $7 / 22 / 08$ 13:09 & 281.3681 & 3 \\
\hline WVE13832-4 & 28620 & WVE13832 & $7 / 23 / 08$ 12:08 & 281.3673 & 4 \\
\hline WVE13832-5 & 28646 & WVE13832 & $7 / 24 / 08$ 12:19 & 281.3636 & 5 \\
\hline WVE13832-6 & 28690 & WVE13832 & $7 / 24 / 08$ 18:10 & 281.3655 & 6 \\
\hline WVE13832-7 & 28721 & WVE13832 & $7 / 25 / 08$ 13:08 & 281.3647 & 7 \\
\hline
\end{tabular}




\begin{tabular}{|c|c|c|c|c|}
\hline WVE13832-8 & 28912 WVE13832 & 7/26/08 15:01 & 281.3633 & 8 \\
\hline WVE13832-9 & 29699|WVE13832 & 8/7/08 20:05 & 281.374 & 9 \\
\hline WVE13832-10 & \begin{tabular}{|l|l|}
29899 & WVE13832
\end{tabular} & 8/14/08 16:21 & 281.3747 & 10 \\
\hline WVE13832-11 & \begin{tabular}{l|l}
30070 & WVE13832 \\
\end{tabular} & $8 / 21 / 08$ 10:30 & 281.376 & 11 \\
\hline WVE13832-12 & 30325|WVE13832 & 8/28/08 10:07 & 281.3826 & 12 \\
\hline WVE13832-13 & \begin{tabular}{l|l|l|}
30548 & WVE13832 \\
\end{tabular} & 9/3/08 15:25 & 281.3847 & 13 \\
\hline WVE13833-1 & $28333 \mid$ WVE13833 & 7/18/08 16:44 & 281.3716 & 1 \\
\hline WVE13833-2 & $28465 \mid$ WVE13833 & $7 / 21 / 08$ 12:26 & 281.3607 & 2 \\
\hline WVE13833-3 & 28547|WVE13833 & 7/22/08 13:09 & 281.3641 & 3 \\
\hline WVE13833-4 & \begin{tabular}{l|l|l|}
28621 & WVE13833 \\
\end{tabular} & $7 / 23 / 08$ 12:09 & 281.361 & 4 \\
\hline WVE13833-5 & 28930 WVE13833 & 7/26/08 15:26 & 281.3311 & 5 \\
\hline WVE13833-6 & $29000 \mid$ WVE13833 & $7 / 27 / 08$ 14:36 & 281.3268 & 6 \\
\hline WVE13833-7 & $29055 \mid$ WVE13833 & 7/28/08 18:33 & 281.3228 & 7 \\
\hline WVE13833-8 & $29123 \mid$ WVE13833 & $7 / 29 / 08$ 12:35 & 281.325 & 8 \\
\hline WVE13833-9 & $29694 \mid$ WVE13833 & 8/7/08 20:01 & 281.3255 & 9 \\
\hline WVE13833-10 & 29858 WVE13833 & $8 / 14 / 08$ 15:50 & 281.3241 & 10 \\
\hline WVE13833-11 & 30028|WVE13833 & 8/21/08 9:57 & 281.3213 & 11 \\
\hline WVE13833-12 & 30283 WVE13833 & 8/28/08 9:34 & 281.3242 & 12 \\
\hline WVE13833-13 & 30504|WVE13833 & 9/3/08 14:40 & 281.3259 & 13 \\
\hline WVE13834-1 & \begin{tabular}{l|l|l|}
28334 & WVE13834 \\
\end{tabular} & $7 / 18 / 08$ 16:45 & 282.7107 & 1 \\
\hline WVE13834-2 & \begin{tabular}{|l|l|}
28466 & WVE13834 \\
\end{tabular} & $7 / 21 / 08$ 12:26 & 282.7022 & 2 \\
\hline WVE13834-3 & \begin{tabular}{l|l|}
28548 & WVE13834 \\
\end{tabular} & 7/22/08 13:10 & 282.7022 & 3 \\
\hline WVE13834-4 & \begin{tabular}{l|l|l|}
28622 & WVE13834 \\
\end{tabular} & $7 / 23 / 08$ 12:10 & 282.7002 & 4 \\
\hline WVE13834-5 & 28948 WVE13834 & $7 / 26 / 08$ 15:40 & 282.6519 & 5 \\
\hline WVE13834-6 & \begin{tabular}{l|l|l|}
28993 & WVE13834 \\
\end{tabular} & $7 / 27 / 0814: 30$ & 282.6487 & 6 \\
\hline WVE13834-7 & \begin{tabular}{l|l|}
29047 & WVE13834 \\
\end{tabular} & $7 / 28 / 08$ 18:27 & 282.6431 & 7 \\
\hline WVE13834-8 & \begin{tabular}{|l|l|}
29115 & WVE13834 \\
\end{tabular} & $7 / 29 / 08$ 12:29 & 282.6452 & 8 \\
\hline WVE13834-9 & \begin{tabular}{|l|l|}
29684 & WVE13834 \\
\end{tabular} & 8/7/08 19:54 & 282.6437 & 9 \\
\hline WVE13834-10 & 29859 WVE13834 & $8 / 14 / 08$ 15:50 & 282.6405 & 10 \\
\hline WVE13834-11 & 30029 WVE13834 & 8/21/08 9:58 & 282.6413 & 11 \\
\hline WVE13834-12 & \begin{tabular}{|l|l|}
30284 & WVE13834 \\
\end{tabular} & 8/28/08 9:35 & 282.6445 & 12 \\
\hline WVE13834-13 & \begin{tabular}{|l|l|}
30505 & WVE13834 \\
\end{tabular} & 9/3/08 14:41 & 282.6468 & 13 \\
\hline WVE13835-1 & 28335 WVE13835 & 7/18/08 16:45 & 285.2495 & 1 \\
\hline WVE13835-2 & \begin{tabular}{l|l|l|}
28467 & WVE13835 \\
\end{tabular} & $7 / 21 / 08$ 12:27 & 285.2391 & 2 \\
\hline WVE13835-3 & 28549 WVE13835 & 7/22/08 13:11 & 285.2407 & 3 \\
\hline WVE13835-4 & \begin{tabular}{l|l|l}
28623 & WVE13835 \\
\end{tabular} & $7 / 23 / 08$ 12:10 & 285.2396 & 4 \\
\hline WVE13835-5 & 28949 WVE13835 & 7/26/08 15:41 & 285.1924 & 5 \\
\hline WVE13835-6 & 28992 WVE13835 & $7 / 27 / 08$ 14:29 & 285.1892 & 6 \\
\hline WVE13835-7 & $29046 \mid$ WVE13835 & 7/28/08 18:27 & 285.1814 & 7 \\
\hline WVE13835-8 & $29114 \mid$ WVE13835 & $7 / 29 / 08$ 12:28 & 285.186 & 8 \\
\hline WVE13835-9 & 29683 WVE13835 & 8/7/08 19:54 & 285.1854 & 9 \\
\hline WVE13835-10 & 29860 WVE13835 & 8/14/08 15:51 & 285.1856 & 10 \\
\hline WVE13835-11 & $30030 \mid$ WVE13835 & 8/21/08 9:59 & 285.1866 & 11 \\
\hline WVE13835-12 & 30285 WVE13835 & 8/28/08 9:36 & 285.1888 & 12 \\
\hline WVE13835-13 & \begin{tabular}{|l|l|}
30506 & WVE13835 \\
\end{tabular} & 9/3/08 14:41 & 285.1881 & 13 \\
\hline WVE13836-1 & 28336 WVE13836 & 7/18/08 16:46 & 300.7863 & 1 \\
\hline WVE13836-2 & \begin{tabular}{l|l|}
28468 & WVE13836 \\
\end{tabular} & $7 / 21 / 08$ 12:28 & 300.7735 & 2 \\
\hline WVE13836-3 & 28550 WVE13836 & 7/22/08 13:12 & 300.7793 & 3 \\
\hline WVE13836-4 & 28624|WVE13836 & 7/23/08 12:11 & 300.7755 & 4 \\
\hline
\end{tabular}




\begin{tabular}{|c|c|c|c|c|}
\hline WVE13836-5 & 28651|WVE13836 & $7 / 24 / 08$ 12:22 & 300.7544 & 5 \\
\hline WVE13836-6 & 28695|WVE13836 & $7 / 24 / 08$ 18:13 & 300.7528 & 6 \\
\hline WVE13836-7 & 28725 WVE13836 & $7 / 25 / 08$ 13:11 & 300.7501 & 7 \\
\hline WVE13836-8 & \begin{tabular}{|l|l|}
28920 & WVE13836 \\
\end{tabular} & 7/26/08 15:06 & 300.7504 & 8 \\
\hline WVE13836-9 & 29690|WVE13836 & 8/7/08 19:58 & 300.7497 & 9 \\
\hline WVE13836-10 & 29863|WVE13836 & $8 / 14 / 08$ 15:52 & 300.7445 & 10 \\
\hline WVE13836-11 & 30033 $\mid$ WVE13836 & $8 / 21 / 08$ 10:00 & 300.7447 & 11 \\
\hline WVE13836-12 & 30288|WVE13836 & 8/28/08 9:37 & 300.7526 & 12 \\
\hline WVE13836-13 & 30509 WVE13836 & 9/3/08 14:45 & 300.7517 & 13 \\
\hline WVE13837-1 & 28337 WVE13837 & 7/18/08 16:47 & 295.9341 & 1 \\
\hline WVE13837-2 & 28469 WVE13837 & $7 / 21 / 08$ 12:29 & 295.9246 & 2 \\
\hline WVE13837-3 & 28551 $\mid$ WVE13837 & $7 / 22 / 08$ 13:13 & 295.9218 & 3 \\
\hline WVE13837-4 & 28625 $\mid$ WVE13837 & $7 / 23 / 08$ 12:12 & 295.9208 & 4 \\
\hline WVE13837-5 & \begin{tabular}{l|l|l|}
28652 & WVE13837
\end{tabular} & $7 / 24 / 08$ 12:23 & 295.9079 & 5 \\
\hline WVE13837-6 & \begin{tabular}{|l|l|}
28696 & WVE13837 \\
\end{tabular} & 7/24/08 18:14 & 295.9057 & 6 \\
\hline WVE13837-7 & \begin{tabular}{|l|l|}
28726 & WVE13837
\end{tabular} & 7/25/08 13:11 & 295.9021 & 7 \\
\hline WVE13837-8 & 28921 WVE13837 & 7/26/08 15:07| & 295.9035 & 8 \\
\hline WVE13837-9 & 29691 WVE13837 & 8/7/08 19:59 & 295.9047 & 9 \\
\hline WVE13837-10 & 29864 WVE13837 & 8/14/08 15:53 & 295.9036 & 10 \\
\hline WVE13837-11 & 30034 WVE13837 & 8/21/08 10:01 & 295.8994 & 11 \\
\hline WVE13837-12 & \begin{tabular}{|l|l|}
30289 & WVE13837 \\
\end{tabular} & 8/28/08 9:38 & 295.9056 & 12 \\
\hline WVE13837-13 & \begin{tabular}{|l|l|}
30510 & WVE13837 \\
\end{tabular} & 9/3/08 14:46 & 295.9037 & 13 \\
\hline WVE13838-1 & 28338 $\mid$ WVE13838 & 7/18/08 16:48 & 282.6694 & 1 \\
\hline WVE13838-2 & \begin{tabular}{|l|l|}
28470 & WVE13838 \\
\end{tabular} & $7 / 21 / 08$ 12:30 & 282.6632 & 2 \\
\hline WVE13838-3 & \begin{tabular}{|l|l|}
28552 & WVE13838 \\
\end{tabular} & $7 / 22 / 08$ 13:14 & 282.6604 & 3 \\
\hline WVE13838-4 & \begin{tabular}{|l|l|}
28626 & WVE13838 \\
\end{tabular} & $7 / 23 / 08$ 12:12 & 282.6598 & 4 \\
\hline WVE13838-5 & 28653 WVE13838 & 7/24/08 12:23 & 282.6588 & 5 \\
\hline WVE13838-6 & 28697|WVE13838 & 7/24/08 18:14 & 282.6591 & 6 \\
\hline WVE13838-7 & 28727 WVE13838 & 7/25/08 13:12 & 282.6512 & 7 \\
\hline WVE13838-8 & 28923|WVE13838 & $7 / 26 / 08$ 15:09 & 282.6569 & 8 \\
\hline WVE13838-9 & 29695 WVE13838 & 8/7/08 20:02 & 282.6561 & 9 \\
\hline WVE13838-10 & 29865|WVE13838 & 8/14/08 15:54 & 282.654 & 10 \\
\hline WVE13838-11 & 30035 WVE13838 & 8/21/08 10:02 & 282.656 & 11 \\
\hline WVE13838-12 & 30290 WVE13838 & 8/28/08 9:38 & 282.6663 & 12 \\
\hline WVE13838-13 & 30511 WVE13838 & 9/3/08 14:47 & 282.6626 & 13 \\
\hline WVE13839-1 & 28339 WVE13839 & 7/18/08 16:49 & 282.5631 & 1 \\
\hline WVE13839-2 & \begin{tabular}{|l|l|}
28471 & WVE13839 \\
\end{tabular} & $7 / 21 / 08$ 12:31 & 282.5045 & 2 \\
\hline WVE13839-3 & 28553|WVE13839 & 7/22/08 13:16 & 282.4996 & 3 \\
\hline WVE13839-4 & 28627 WVE13839 & $7 / 23 / 08$ 12:13 & 282.5024 & 4 \\
\hline WVE13940-1 & \begin{tabular}{|l|l|}
28340 & WVE13940 \\
\end{tabular} & $7 / 18 / 08$ 16:50 & 297.035 & 1 \\
\hline WVE13940-2 & \begin{tabular}{l|l|l|}
28472 & WVE13940
\end{tabular} & $7 / 21 / 08$ 12:32 & 297.0321 & 2 \\
\hline WVE13940-3 & 28554 WVE13940 & 7/22/08 13:17 & 297.029 & 3 \\
\hline WVE13940-4 & \begin{tabular}{|l|l|}
28628 & WVE13940 \\
\end{tabular} & $7 / 23 / 08$ 12:14 & 297.028 & 4 \\
\hline WVE13940-5 & \begin{tabular}{|l|l|}
28647 & WVE13940 \\
\end{tabular} & $7 / 24 / 08$ 12:19 & 297.0292 & 5 \\
\hline WVE13940-6 & 28691 WVE13940 & 7/24/08 18:11 & 297.0276 & 6 \\
\hline WVE13940-7 & \begin{tabular}{|l|l|}
28722 & WVE13940 \\
\end{tabular} & 7/25/08 13:09 & 297.026 & 7 \\
\hline WVE13940-8 & \begin{tabular}{l|l|l|}
28911 & WVE13940 \\
\end{tabular} & 7/26/08 15:00 & 297.0223 & 8 \\
\hline WVE13940-9 & 29698 $\mid$ WVE13940 & 8/7/08 20:04 & 297.0288 & 9 \\
\hline WVE13940-10 & \begin{tabular}{|l|l|l|}
29900 & WVE13940 \\
\end{tabular} & 8/14/08 16:22 & 297.0347 & 10 \\
\hline
\end{tabular}




\begin{tabular}{|c|c|c|c|c|}
\hline WVE13940-11 & \begin{tabular}{l|l|l|}
30071 & WVE13940
\end{tabular} & 8/21/08 10:31 & 297.0333 & 11 \\
\hline WVE13940-12 & \begin{tabular}{l|l|}
30326 & WVE13940 \\
\end{tabular} & 8/28/08 10:07| & 297.0379 & 12 \\
\hline WVE13940-13 & \begin{tabular}{|c|c|}
30547 & WVE13940
\end{tabular} & 9/3/08 15:24 & 297.0407 & 13 \\
\hline WVE13941-1 & \begin{tabular}{l|l}
28341 & WVE13941
\end{tabular} & 7/18/08 16:51 & 294.4728 & 1 \\
\hline WVE13941-2 & 28473|WVE13941 & $7 / 21 / 08$ 12:33 & 294.4699 & 2 \\
\hline WVE13941-3 & 28555|WVE13941 & 7/22/08 13:18 & 294.4659 & 3 \\
\hline WVE13941-4 & 28629 $\mid$ WVE13941 & $7 / 23 / 08$ 12:15 & 294.468 & 4 \\
\hline WVE13941-5 & \begin{tabular}{l|l|}
28654 & WVE13941
\end{tabular} & 7/24/08 12:24 & 294.46 & 5 \\
\hline WVE13941-6 & \begin{tabular}{|l|l|}
28698 & WVE13941 \\
\end{tabular} & 7/24/08 18:15 & 294.4594 & 6 \\
\hline WVE13941-7 & 28728 $\mid$ WVE13941 & 7/25/08 13:13 & 294.4552 & 7 \\
\hline WVE13941-8 & 28924 $\mid$ WVE13941 & $7 / 26 / 08$ 15:09 & 294.4603 & 8 \\
\hline WVE13941-9 & \begin{tabular}{|l|l|} 
29696 & WVE13941 \\
\end{tabular} & 8/7/08 20:03 & 294.4646 & 9 \\
\hline WVE13942-1 & 28344 WVE13942 & $7 / 18 / 08$ 16:53 & 296.4613 & 1 \\
\hline WVE13942-2 & \begin{tabular}{l|l|}
28477 & WVE13942 \\
\end{tabular} & $7 / 21 / 08$ 12:37 & 296.4562 & 2 \\
\hline WVE13942-3 & 28558|WVE13942 & $7 / 22 / 08$ 13:20 & 296.4578 & 3 \\
\hline WVE13942-4 & \begin{tabular}{l|l|}
28632 & WVE13942 \\
\end{tabular} & $7 / 23 / 08$ 12:16 & 296.4587 & 4 \\
\hline WVE13942-5 & 29289|WVE13942 & $7 / 31 / 08$ 15:25 & 295.5382 & 5 \\
\hline WVE13942-6 & 29315|WVE13942 & 8/1/08 13:44 & 295.5392 & 6 \\
\hline WVE13942-7 & 29384|WVE13942 & $8 / 2 / 08$ 14:32 & 295.5463 & 7 \\
\hline WVE13942-8 & \begin{tabular}{|l|l|}
29430 & WVE13942 \\
\end{tabular} & 8/3/08 12:38 & 295.5543 & 8 \\
\hline WVE13942-9 & \begin{tabular}{l|l|}
29706 & WVE13942
\end{tabular} & 8/7/08 20:09 & 295.576 & 9 \\
\hline WVE13942-10 & \begin{tabular}{|l|l|} 
29866 & WVE13942 \\
\end{tabular} & $8 / 14 / 08$ 15:55 & 295.6286 & 10 \\
\hline WVE13942-11 & \begin{tabular}{l|l|l}
30036 & WVE13942
\end{tabular} & 8/21/08 10:03 & 295.6595 & 11 \\
\hline WVE13942-12 & 30291 $\mid$ WVE13942 & 8/28/08 9:39 & 295.6903 & 12 \\
\hline WVE13942-13 & 30512|WVE13942 & 9/3/08 14:48 & 295.7179 & 13 \\
\hline WVE13943-1 & 28345 WVE13943 & $7 / 18 / 08$ 16:54 & 298.105 & 1 \\
\hline WVE13943-2 & 28478|WVE13943 & $7 / 21 / 08$ 12:38 & 298.093 & 2 \\
\hline WVE13943-3 & $28559 \mid$ WVE13943 & 7/22/08 13:21 & 298.0957 & 3 \\
\hline WVE13943-4 & 28633|WVE13943 & $7 / 23 / 08$ 12:17 & 298.0999 & 4 \\
\hline WVE13943-5 & 29290|WVE13943 & $7 / 31 / 08$ 15:26 & 297.2031 & 5 \\
\hline WVE13943-6 & 29316|WVE13943 & 8/1/08 13:45 & 297.2035 & 6 \\
\hline WVE13943-7 & 29385|WVE13943 & 8/2/08 14:33 & 297.2148 & 7 \\
\hline WVE13943-8 & 29431 $\mid$ WVE13943 & $8 / 3 / 08$ 12:39 & 297.2245 & 8 \\
\hline WVE13943-9 & \begin{tabular}{|l|l|l|}
29705 & WVE13943
\end{tabular} & 8/7/08 20:09 & 297.2404 & 9 \\
\hline WVE13943-10 & \begin{tabular}{|l|l|}
29894 & WVE13943 \\
\end{tabular} & 8/14/08 16:18 & 297.2877 & 10 \\
\hline WVE13943-11 & 30064 $\mid$ WVE13943 & $8 / 21 / 08$ 10:26 & 297.3081 & 11 \\
\hline WVE13943-12 & 30319|WVE13943 & 8/28/08 10:02 & 297.3231 & 12 \\
\hline WVE13943-13 & 30540 WVE13943 & 9/3/08 15:18 & 297.3357 & 13 \\
\hline WVE13944-1 & \begin{tabular}{|l|l|}
28290 & WVE13944 \\
\end{tabular} & $7 / 18 / 08$ 15:59 & 311.1093 & 1 \\
\hline WVE13944-2 & \begin{tabular}{l|l|l|}
28422 & WVE13944 \\
\end{tabular} & $7 / 21 / 08$ 11:45 & 311.0998 & 2 \\
\hline WVE13944-3 & \begin{tabular}{l|l|}
28504 & WVE13944
\end{tabular} & $7 / 22 / 08$ 12:19 & 311.0944 & 3 \\
\hline WVE13944-4 & 28578 $\mid$ WVE13944 & $7 / 23 / 08$ 11:35 & 311.0968 & 4 \\
\hline WVE13944-5 & 29291 $\mid$ WVE13944 & 7/31/08 15:27 & 310.2406 & 5 \\
\hline WVE13944-6 & \begin{tabular}{l|l|}
29317 & WVE13944 \\
\end{tabular} & 8/1/08 13:45 & 310.2436 & 6 \\
\hline WVE13944-7 & \begin{tabular}{|l|l|} 
29386 & WVE13944
\end{tabular} & 8/2/08 14:33 & 310.261 & 7 \\
\hline WVE13944-8 & \begin{tabular}{|l|l|}
29432 & WVE13944 \\
\end{tabular} & $8 / 3 / 08$ 12:39 & 310.2682 & 8 \\
\hline WVE13944-9 & \begin{tabular}{l|l|l|}
29707 & WVE13944
\end{tabular} & 8/7/08 20:10 & 310.2929 & 9 \\
\hline WVE13944-10 & \begin{tabular}{|l|l|}
29895 & WVE13944
\end{tabular} & 8/14/08 16:19 & 310.3191 & 10 \\
\hline WVE13944-11 & 30065 WVE13944 & 8/21/08 10:27| & 310.3394 & 11 \\
\hline
\end{tabular}




\begin{tabular}{|c|c|c|c|c|}
\hline WVE13944-12 & 30320 $\mid$ WVE13944 & 8/28/08 10:03 & 310.3561 & 12 \\
\hline WVE13944-13 & \begin{tabular}{l|l|}
30541 & WVE13944 \\
\end{tabular} & 9/3/08 15:19 & 310.3764 & 13 \\
\hline WVE13945-1 & \begin{tabular}{|l|l|}
28291 & WVE13945
\end{tabular} & $7 / 18 / 08$ 16:00 & 309.386 & 1 \\
\hline WVE13945-2 & \begin{tabular}{l|l|} 
28423 & WVE13945
\end{tabular} & $7 / 21 / 08$ 11:46 & 309.3751 & 2 \\
\hline WVE13945-3 & 28505|WVE13945 & $7 / 22 / 08$ 12:20 & 309.3698 & 3 \\
\hline WVE13945-4 & $28579 \mid$ WVE13945 & $7 / 23 / 08$ 11:36 & 309.3704 & 4 \\
\hline WVE13945-5 & \begin{tabular}{l|l|l|}
29292 & WVE13945
\end{tabular} & 7/31/08 15:27| & 308.4344 & 5 \\
\hline WVE13945-6 & 29318|WVE13945 & $8 / 1 / 08$ 13:46 & 308.4346 & 6 \\
\hline WVE13945-7 & \begin{tabular}{|l|l|}
29387 & WVE13945 \\
\end{tabular} & $8 / 2 / 0814: 34$ & 308.4401 & 7 \\
\hline WVE13945-8 & 29433 $\mid$ WVE13945 & $8 / 3 / 08$ 12:40 & 308.4479 & 8 \\
\hline WVE13945-9 & 29708 $\mid$ WVE13945 & 8/7/08 20:11 & 308.4691 & 9 \\
\hline WVE13945-10 & \begin{tabular}{|l|l|}
29896 & WVE13945
\end{tabular} & 8/14/08 16:19 & 308.5141 & 10 \\
\hline WVE13945-11 & \begin{tabular}{|l|l|l|}
30066 & WVE13945 \\
\end{tabular} & $8 / 21 / 08$ 10:27| & 308.5502 & 11 \\
\hline WVE13945-12 & 30321 $\mid$ WVE13945 & $8 / 28 / 08$ 10:04 & 308.5725 & 12 \\
\hline WVE13945-13 & \begin{tabular}{l|l|l}
30542 & WVE13945 \\
\end{tabular} & 9/3/08 15:20 & 308.5745 & 13 \\
\hline WVE13946-1 & \begin{tabular}{l|l|}
28292 & WVE13946
\end{tabular} & 7/18/08 16:01 & 301.3783 & 1 \\
\hline WVE13946-2 & 28424 $\mid$ WVE13946 & 7/21/08 11:47 & 301.3679 & 2 \\
\hline WVE13946-3 & \begin{tabular}{l|l|}
28506 & WVE13946
\end{tabular} & $7 / 22 / 08$ 12:21 & 301.3634 & 3 \\
\hline WVE13946-4 & 28580 $\mid$ WVE13946 & $7 / 23 / 08$ 11:36 & 301.3634 & 4 \\
\hline WVE13946-5 & \begin{tabular}{|l|l|}
29293 & WVE13946 \\
\end{tabular} & 7/31/08 15:28 & 300.4862 & 5 \\
\hline WVE13946-6 & 29319|WVE13946 & 8/1/08 13:47 & 300.4926 & 6 \\
\hline WVE13946-7 & 29388|WVE13946 & $8 / 2 / 0814: 35$ & 300.5035 & 7 \\
\hline WVE13946-8 & \begin{tabular}{l|l|}
29434 & WVE13946
\end{tabular} & $8 / 3 / 08$ 12:41 & 300.5128 & 8 \\
\hline WVE13946-9 & $29709 \mid$ WVE13946 & 8/7/08 20:12 & 300.5355 & 9 \\
\hline WVE13946-10 & 29897 WVE13946 & $8 / 14 / 08$ 16:20 & 300.5651 & 10 \\
\hline WVE13946-11 & \begin{tabular}{l|l|l|}
30067 & WVE13946
\end{tabular} & 8/21/08 10:28 & 300.601 & 11 \\
\hline WVE13946-12 & \begin{tabular}{l|l|l}
30322 & WVE13946
\end{tabular} & 8/28/08 10:05 & 300.6303 & 12 \\
\hline WVE13946-13 & \begin{tabular}{l|l|}
30543 & WVE13946
\end{tabular} & 9/3/08 15:21 & 300.6533 & 13 \\
\hline WVE13947-1 & \begin{tabular}{|l|l|}
28293 & WVE13947 \\
\end{tabular} & 7/18/08 16:01 & 291.9714 & 1 \\
\hline WVE13947-2 & \begin{tabular}{|l|l|}
28425 & WVE13947
\end{tabular} & 7/21/08 11:47 & 291.9607 & 2 \\
\hline WVE13947-3 & \begin{tabular}{|l|l|}
28507 & WVE13947 \\
\end{tabular} & $7 / 22 / 08$ 12:22 & 291.9623 & 3 \\
\hline WVE13947-4 & $28581 \mid$ WVE13947 & $7 / 23 / 08$ 11:37 & 291.9593 & 4 \\
\hline WVE13947-5 & 29294 $\mid$ WVE13947 & 7/31/08 15:28 & 290.8307 & 5 \\
\hline WVE13947-6 & \begin{tabular}{l|l|l} 
29320 & WVE13947
\end{tabular} & $8 / 1 / 08$ 13:48 & 290.8468 & 6 \\
\hline WVE13947-7 & \begin{tabular}{|l|l|}
29389 & WVE13947 \\
\end{tabular} & $8 / 2 / 0814: 36$ & 290.8609 & 7 \\
\hline WVE13947-8 & $29439 \mid$ WVE13947 & $8 / 3 / 08$ 12:43 & 290.8714 & 8 \\
\hline WVE13947-9 & \begin{tabular}{|l|l|} 
29716 & WVE13947 \\
\end{tabular} & 8/7/08 20:17 & 290.8943 & 9 \\
\hline WVE13947-10 & 29898|WVE13947 & 8/14/08 16:21 & 290.9263 & 10 \\
\hline WVE13947-11 & \begin{tabular}{|l|l|l|}
30068 & WVE13947 \\
\end{tabular} & 8/21/08 10:29 & 290.9615 & 11 \\
\hline WVE13947-12 & 30323|WVE13947 & 8/28/08 10:05 & 291.0005 & 12 \\
\hline WVE13947-13 & \begin{tabular}{l|l|l}
30544 & WVE13947 \\
\end{tabular} & 9/3/08 15:22 & 291.0269 & 13 \\
\hline WVE14338-1 & 28284 WVE14338 & 7/18/08 15:51 & 296.5318 & 1 \\
\hline WVE14338-2 & \begin{tabular}{|l|l|}
28416 & WVE14338 \\
\end{tabular} & $7 / 21 / 08$ 11:40 & 296.5257 & 2 \\
\hline WVE14338-3 & 28498|WVE14338 & $7 / 22 / 08$ 12:13 & 296.5254 & 3 \\
\hline WVE14338-4 & \begin{tabular}{l|l|}
28572 & WVE14338 \\
\end{tabular} & 7/23/08 11:32 & 296.5279 & 4 \\
\hline WVE14338-5 & 28937|WVE14338 & $7 / 26 / 08$ 15:32 & 296.4449 & 5 \\
\hline WVE14338-6 & \begin{tabular}{|l|l|}
29003 & WVE14338
\end{tabular} & 7/27/08 14:38 & 296.4424 & 6 \\
\hline WVE14338-7 & 29058|WVE14338 & $7 / 28 / 08$ 18:35 & 296.4378 & 7 \\
\hline WVE14338-8 & \begin{tabular}{l|l|}
29126 & WVE14338 \\
\end{tabular} & 7/29/08 12:37 & 296.439 & 8 \\
\hline
\end{tabular}




\begin{tabular}{|c|c|c|c|c|}
\hline WVE14338-9 & \begin{tabular}{l|l|}
29692 & WVE14338
\end{tabular} & 8/7/08 20:00 & 296.4377 & 9 \\
\hline WVE14338-10 & \begin{tabular}{l|l|}
29883 & WVE14338 \\
\end{tabular} & 8/14/08 16:07 & 296.4388 & 10 \\
\hline WVE14338-11 & \begin{tabular}{|c|c|c|}
3005 & WVE14338
\end{tabular} & 8/21/08 10:16 & 296.4381 & 11 \\
\hline WVE14338-12 & 30308|WVE14338 & 8/28/08 9:53 & 296.4451 & 12 \\
\hline WVE14338-13 & $30529 \mid$ WVE14338 & 9/3/08 15:07 & 296.4456 & 13 \\
\hline WVE14339-1 & 28285|WVE14339 & 7/18/08 15:52 & 294.5253 & 1 \\
\hline WVE14339-2 & 28417|WVE14339 & 7/21/08 11:41 & 294.5175 & 2 \\
\hline WVE14339-3 & 28499|WVE14339 & $7 / 22 / 08$ 12:14 & 294.5169 & 3 \\
\hline WVE14339-4 & \begin{tabular}{|l|l|}
28573 & WVE14339 \\
\end{tabular} & 7/23/08 11:32 & 294.5211 & 4 \\
\hline WVE14339-5 & \begin{tabular}{|l|l|}
28945 & WVE14339
\end{tabular} & 7/26/08 15:38 & 294.4577 & 5 \\
\hline WVE14339-6 & \begin{tabular}{|l|l|} 
28996 & WVE14339
\end{tabular} & $7 / 27 / 08$ 14:32 & 294.456 & 6 \\
\hline WVE14339-7 & 29050 WVE14339 & $7 / 28 / 08$ 18:29 & 294.4507 & 7 \\
\hline WVE14339-8 & 29118|WVE14339 & $7 / 29 / 08$ 12:31 & 294.4549 & 8 \\
\hline WVE14339-9 & \begin{tabular}{|l|l|}
29687 & WVE14339
\end{tabular} & $8 / 7 / 08$ 19:56 & 294.4559 & 9 \\
\hline WVE14339-10 & 29884 $\mid$ WVE14339 & 8/14/08 16:08 & 294.4549 & 10 \\
\hline WVE14339-11 & \begin{tabular}{l|l|l}
30054 & WVE14339
\end{tabular} & $8 / 21 / 08$ 10:17 & 294.4597 & 11 \\
\hline WVE14339-12 & 30309|WVE14339 & 8/28/08 9:54 & 294.4621 & 12 \\
\hline WVE14339-13 & 30530|WVE14339 & 9/3/08 15:08 & 294.4664 & 13 \\
\hline WVE14340-1 & 28286 $\mid$ WVE14340 & 7/18/08 15:53 & 297.5643 & 1 \\
\hline WVE14340-2 & \begin{tabular}{|l|l|}
28418 & WVE14340 \\
\end{tabular} & 7/21/08 11:42 & 297.5555 & 2 \\
\hline WVE14340-3 & 28500 $\mid$ WVE14340 & $7 / 22 / 08$ 12:15 & 297.5556 & 3 \\
\hline WVE14340-4 & \begin{tabular}{l|l|l}
28574 & WVE14340 \\
\end{tabular} & $7 / 23 / 08$ 11:33 & 297.5541 & 4 \\
\hline WVE14340-5 & 28947 $\mid$ WVE14340 & $7 / 26 / 08$ 15:39 & 297.4931 & 5 \\
\hline WVE14340-6 & 28994 $\mid$ WVE14340 & 7/27/08 14:31 & 297.4959 & 6 \\
\hline WVE14340-7 & 29048|WVE14340 & $7 / 28 / 08$ 18:28 & 297.4928 & 7 \\
\hline WVE14340-8 & \begin{tabular}{|l|l|} 
29116 & WVE14340 \\
\end{tabular} & $7 / 29 / 08$ 12:30 & 297.4913 & 8 \\
\hline WVE14340-9 & 29685|WVE14340 & 8/7/08 19:55 & 297.4908 & 9 \\
\hline WVE14340-10 & 29885|WVE14340 & 8/14/08 16:09 & 297.4911 & 10 \\
\hline WVE14340-11 & $30055 \mid$ WVE14340 & 8/21/08 10:18 & 297.4986 & 11 \\
\hline WVE14340-12 & \begin{tabular}{|l|l|}
30310 & WVE14340
\end{tabular} & 8/28/08 9:54 & 297.5036 & 12 \\
\hline WVE14340-13 & 30531|WVE14340 & 9/3/08 15:09 & 297.5047 & 13 \\
\hline WVE14341-1 & 28287 $\mid$ WVE14341 & $7 / 18 / 08$ 15:54 & 281.7495 & 1 \\
\hline WVE14341-2 & 28419 $\mid$ WVE14341 & $7 / 21 / 08$ 11:43 & 281.7391 & 2 \\
\hline WVE14341-3 & \begin{tabular}{l|l}
28501 & WVE14341
\end{tabular} & $7 / 22 / 08$ 12:16 & 281.7365 & 3 \\
\hline WVE14341-4 & \begin{tabular}{|l|l|}
28575 & WVE14341 \\
\end{tabular} & 7/23/08 11:33 & 281.7385 & 4 \\
\hline WVE14341-5 & \begin{tabular}{l|l|} 
28946 & WVE14341
\end{tabular} & 7/26/08 15:38 & 281.6996 & 5 \\
\hline WVE14341-6 & 28995|WVE14341 & $7 / 27 / 08$ 14:31 & 281.7007 & 6 \\
\hline WVE14341-7 & 29049 WVE14341 & $7 / 28 / 08$ 18:29 & 281.6937 & 7 \\
\hline WVE14341-8 & \begin{tabular}{|l|l|}
29117 & WVE14341 \\
\end{tabular} & $7 / 29 / 08$ 12:30 & 281.697 & 8 \\
\hline WVE14341-9 & \begin{tabular}{|l|l|} 
29686 & WVE14341 \\
\end{tabular} & 8/7/08 19:56 & 281.6962 & 9 \\
\hline WVE14341-10 & \begin{tabular}{|l|l|}
29886 & WVE14341 \\
\end{tabular} & 8/14/08 16:10 & 281.7014 & 10 \\
\hline WVE14341-11 & \begin{tabular}{|l|l|}
30056 & WVE14341 \\
\end{tabular} & 8/21/08 10:19 & 281.7014 & 11 \\
\hline WVE14341-12 & 30311|WVE14341 & 8/28/08 9:55 & 281.7054 & 12 \\
\hline WVE14341-13 & \begin{tabular}{l|l|l}
30532 & WVE14341 \\
\end{tabular} & 9/3/08 15:09 & 281.7106 & 13 \\
\hline WVE14342-1 & \begin{tabular}{l|l|}
28288 & WVE14342
\end{tabular} & $7 / 18 / 08$ 15:54 & 279.4554 & 1 \\
\hline WVE14342-2 & \begin{tabular}{|l|l|}
28420 & WVE14342 \\
\end{tabular} & 7/21/08 11:43 & 279.4424 & 2 \\
\hline WVE14342-3 & \begin{tabular}{l|l|}
28502 & WVE14342
\end{tabular} & $7 / 22 / 08$ 12:17 & 279.4451 & 3 \\
\hline WVE14342-4 & \begin{tabular}{|l|l|}
28576 & WVE14342 \\
\end{tabular} & $7 / 23 / 08$ 11:34 & 279.44 & 4 \\
\hline WVE14342-5 & \begin{tabular}{l|l|l|} 
28954 & WVE14342 \\
\end{tabular} & 7/26/08 15:44 & 279.421 & 5 \\
\hline
\end{tabular}




\begin{tabular}{|c|c|c|c|c|}
\hline WVE14342-6 & 28983 $\mid$ WVE14342 & $7 / 27 / 08$ 14:23 & 279.4166 & 6 \\
\hline WVE14342-7 & \begin{tabular}{l|l|l|}
29037 & WVE14342 \\
\end{tabular} & 7/28/08 18:20 & 279.4147 & 7 \\
\hline WVE14342-8 & \begin{tabular}{|c|c|}
29105 & WVE14342
\end{tabular} & $7 / 29 / 08$ 12:22 & 279.414 & 8 \\
\hline WVE14342-9 & \begin{tabular}{l|l|}
29679 & WVE14342 \\
\end{tabular} & 8/7/08 19:50 & 279.4186 & 9 \\
\hline WVE14342-10 & \begin{tabular}{l|l|l|}
29847 & WVE14342 \\
\end{tabular} & 8/14/08 15:41 & 279.4172 & 10 \\
\hline WVE14342-11 & \begin{tabular}{l|l|l|}
30017 & WVE14342 \\
\end{tabular} & $8 / 21 / 08$ 9:50 & 279.4143 & 11 \\
\hline WVE14342-12 & \begin{tabular}{l|l|l|}
30272 & WVE14342 \\
\end{tabular} & 8/28/08 9:26 & 279.42 & 12 \\
\hline WVE14342-13 & \begin{tabular}{|c|c|}
30493 & WVE14342 \\
\end{tabular} & 9/3/08 14:31 & 279.4219 & 13 \\
\hline WVE14343-1 & 28289 WVE14343 & $7 / 18 / 08$ 15:56 & 276.6593 & 1 \\
\hline WVE14343-2 & \begin{tabular}{l|l|l|}
28421 & WVE14343 \\
\end{tabular} & $7 / 21 / 08$ 11:44 & 276.6442 & 2 \\
\hline WVE14343-3 & 28503 WVE14343 & $7 / 22 / 08$ 12:18 & 276.6389 & 3 \\
\hline WVE14343-4 & \begin{tabular}{l|l|l|}
28577 & WVE14343
\end{tabular} & $7 / 23 / 08$ 11:35 & 276.64 & 4 \\
\hline WVE14343-5 & 28955 WVE14343 & 7/26/08 15:44 & 276.6211 & 5 \\
\hline WVE14343-6 & $28984 \mid$ WVE14343 & $7 / 27 / 08$ 14:23 & 276.6271 & 6 \\
\hline WVE14343-7 & 29038 WVE14343 & $7 / 28 / 08$ 18:21 & 276.6168 & 7 \\
\hline WVE14343-8 & \begin{tabular}{|l|l|}
29106 & WVE14343 \\
\end{tabular} & $7 / 29 / 08$ 12:23 & 276.6147 & 8 \\
\hline WVE14343-9 & 29678 WVE14343 & $8 / 7 / 08$ 19:50 & 276.6152 & 9 \\
\hline WVE14343-10 & \begin{tabular}{|l|l|}
29848 & WVE14343 \\
\end{tabular} & $8 / 14 / 08$ 15:42 & 276.6109 & 10 \\
\hline WVE14343-11 & \begin{tabular}{|l|l|}
30018 & WVE14343 \\
\end{tabular} & 8/21/08 9:50 & 276.6049 & 11 \\
\hline WVE14343-12 & 30273|WVE14343 & 8/28/08 9:27 & 276.6062 & 12 \\
\hline WVE14343-13 & 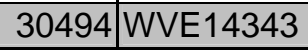 & 9/3/08 14:31 & 276.6083 & 13 \\
\hline WVE14344-1 & \begin{tabular}{l|l|}
28296 & WVE14344 \\
\end{tabular} & 7/18/08 16:07 & 294.8828 & 1 \\
\hline WVE14344-2 & \begin{tabular}{l|l|}
28428 & WVE14344 \\
\end{tabular} & $7 / 21 / 08$ 11:53 & 294.8833 & 2 \\
\hline WVE14344-3 & 28510 $\mid$ WVE14344 & $7 / 22 / 08$ 12:26 & 294.8822 & 3 \\
\hline WVE14344-4 & \begin{tabular}{l|l|l|}
28584 & WVE14344 \\
\end{tabular} & $7 / 23 / 0811: 40$ & 294.8843 & 4 \\
\hline WVE14344-5 & \begin{tabular}{l|l|}
28956 & WVE14344 \\
\end{tabular} & $7 / 26 / 08$ 15:45 & 294.8567 & 5 \\
\hline WVE14344-6 & \begin{tabular}{|l|l|}
28976 & WVE14344 \\
\end{tabular} & $7 / 27 / 08$ 14:18 & 294.8533 & 6 \\
\hline WVE14344-7 & \begin{tabular}{|l|l|}
29030 & WVE14344 \\
\end{tabular} & 7/28/08 18:16 & 294.8498 & 7 \\
\hline WVE14344-8 & \begin{tabular}{|l|l|}
29098 & WVE14344 \\
\end{tabular} & 7/29/08 12:18 & 294.8512 & 8 \\
\hline WVE14344-9 & \begin{tabular}{l|l|}
29682 & WVE14344 \\
\end{tabular} & 8/7/08 19:53 & 294.8473 & 9 \\
\hline WVE14344-10 & 29849 WVE14344 & $8 / 14 / 08$ 15:43 & 294.8379 & 10 \\
\hline WVE14344-11 & \begin{tabular}{l|l|l|}
30019 & WVE14344 \\
\end{tabular} & 8/21/08 9:51 & 294.8341 & 11 \\
\hline WVE14344-12 & 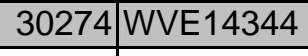 & $8 / 28 / 089: 28$ & 294.8381 & 12 \\
\hline WVE14344-13 & \begin{tabular}{l|l|l|}
30495 & WVE14344 \\
\end{tabular} & 9/3/08 14:32 & 294.8423 & 13 \\
\hline WVE14345-1 & 28297 WVE14345 & 7/18/08 16:08 & 295.0351 & 1 \\
\hline WVE14345-2 & \begin{tabular}{l|l|l|}
28429 & WVE14345 \\
\end{tabular} & $7 / 21 / 08$ 11:54 & 295.0196 & 2 \\
\hline WVE14345-3 & \begin{tabular}{l|l|l|}
28511 & WVE14345 \\
\end{tabular} & $7 / 22 / 08$ 12:27 & 295.0316 & 3 \\
\hline WVE14345-4 & $28585 \mid$ WVE14345 & $7 / 23 / 08$ 11:41 & 295.0198 & 4 \\
\hline WVE14345-5 & $\begin{array}{l}28952 \\
\text { WVE14345 }\end{array}$ & $7 / 26 / 08$ 15:42 & 295.005 & 5 \\
\hline WVE14345-6 & 28981 WVE14345 & $7 / 27 / 08$ 14:22 & 295 & 6 \\
\hline WVE14345-7 & 29035 WVE14345 & 7/28/08 18:19 & 294.9981 & 7 \\
\hline WVE14345-8 & 29103 $\mid$ WVE14345 & 7/29/08 12:21 & 295.0005 & 8 \\
\hline WVE14345-9 & \begin{tabular}{|l|l|}
29681 & WVE14345 \\
\end{tabular} & $8 / 7 / 08$ 19:52 & 294.9947 & 9 \\
\hline WVE14345-10 & $29850 \mid$ WVE14345 & 8/14/08 15:44 & 294.9871 & 10 \\
\hline WVE14345-11 & \begin{tabular}{|l|l|}
30020 & WVE14345 \\
\end{tabular} & 8/21/08 9:52 & 294.9862 & 11 \\
\hline WVE14345-12 & 30275 WVE14345 & 8/28/08 9:28 & 294.9889 & 12 \\
\hline WVE14345-13 & \begin{tabular}{|l|l|}
30496 & WVE14345 \\
\end{tabular} & 9/3/08 14:33 & 294.9944 & 13 \\
\hline WVE14695-1 & \begin{tabular}{l|l|l|}
27731 & WVE14695 \\
\end{tabular} & 7/11/08 18:30 & 291.6971 & 1 \\
\hline WVE14695-2 & \begin{tabular}{|l|l|}
27778 & WVE14695 \\
\end{tabular} & 7/12/08 13:07 & 291.6778 & 2 \\
\hline
\end{tabular}




\begin{tabular}{|c|c|c|c|c|}
\hline WVE14695-3 & \begin{tabular}{l|l|l}
27806 & WVE14695
\end{tabular} & $7 / 13 / 08$ 15:02 & 291.6716 & 3 \\
\hline WVE14695-4 & \begin{tabular}{l|l|l|}
27937 & WVE14695
\end{tabular} & $7 / 14 / 08$ 14:24 & 291.6748 & 4 \\
\hline WVE14695-5 & \begin{tabular}{|l|l|}
27996 & WVE14695
\end{tabular} & $7 / 15 / 08$ 13:33 & 291.697 & 5 \\
\hline WVE14695-6 & \begin{tabular}{l|l|}
28022 & WVE14695
\end{tabular} & 7/15/08 19:20 & 291.692 & 6 \\
\hline WVE14695-7 & 28085|WVE14695 & 7/16/08 19:24 & 291.6776 & 7 \\
\hline WVE14695-8 & 28119|WVE14695 & 7/17/08 14:43 & 291.6754 & 8 \\
\hline WVE14695-9 & 28264|WVE14695 & 7/18/08 15:32 & 291.6749 & 9 \\
\hline WVE14695-10 & \begin{tabular}{l|l|} 
28396 & WVE14695
\end{tabular} & 7/21/08 11:22 & 291.6675 & 10 \\
\hline WVE14695-11 & \begin{tabular}{|l|l|}
28744 & WVE14695 \\
\end{tabular} & 7/25/08 13:25 & 291.6701 & 11 \\
\hline WVE14695-12 & \begin{tabular}{|l|l|}
29259 & WVE14695
\end{tabular} & 7/30/08 12:33 & 291.6782 & 12 \\
\hline WVE14695-13 & 29658|WVE14695 & 8/7/08 19:34 & 291.6824 & 13 \\
\hline WVE14695-14 & 29930 WVE14695 & $8 / 14 / 08$ 16:42 & 291.6804 & 14 \\
\hline WVE14695-15 & $30101 \mid$ WVE14695 & $8 / 21 / 08$ 10:55 & 291.6831 & 15 \\
\hline WVE14695-16 & $30355 \mid$ WVE14695 & $8 / 28 / 08$ 10:33 & 291.6842 & 16 \\
\hline WVE14695-17 & 30581 $\mid$ WVE14695 & 9/3/08 17:01 & 291.6894 & 17 \\
\hline WVE14696-1 & \begin{tabular}{l|l|l|}
27732 & WVE14696
\end{tabular} & 7/11/08 18:31 & 291.4131 & 1 \\
\hline WVE14696-2 & $27769 \mid$ WVE14696 & 7/12/08 13:01 & 291.401 & 2 \\
\hline WVE14696-3 & 27807|WVE14696 & 7/13/08 15:03 & 291.3959 & 3 \\
\hline WVE14696-4 & 27938|WVE14696 & $7 / 14 / 08$ 14:25 & 291.3982 & 4 \\
\hline WVE14696-5 & \begin{tabular}{|l|l|}
27987 & WVE14696 \\
\end{tabular} & 7/15/08 13:26 & 291.3901 & 5 \\
\hline WVE14696-6 & 28023|WVE14696 & 7/15/08 19:20 & 291.3965 & 6 \\
\hline WVE14696-7 & \begin{tabular}{|l|l|} 
28086 & WVE14696 \\
\end{tabular} & $7 / 16 / 08$ 19:25 & 291.3936 & 7 \\
\hline WVE14696-8 & 28120 WVE14696 & $7 / 17 / 08$ 14:43 & 291.3931 & 8 \\
\hline WVE14696-9 & \begin{tabular}{l|l}
28266 & WVE14696
\end{tabular} & 7/18/08 15:33 & 291.397 & 9 \\
\hline WVE14696-10 & 28397|WVE14696 & $7 / 21 / 08$ 11:22 & 291.3894 & 10 \\
\hline WVE14696-11 & 28745 WVE14696 & $7 / 25 / 08$ 13:26 & 291.3927 & 11 \\
\hline WVE14696-12 & 29260|WVE14696 & $7 / 30 / 08$ 12:34 & 291.3986 & 12 \\
\hline WVE14696-13 & 29659 WVE14696 & 8/7/08 19:35 & 291.398 & 13 \\
\hline WVE14696-14 & 29935|WVE14696 & $8 / 14 / 08$ 16:46 & 291.3998 & 14 \\
\hline WVE14696-15 & \begin{tabular}{|l|l|}
30106 & WVE14696
\end{tabular} & 8/21/08 10:58 & 291.4009 & 15 \\
\hline WVE14696-16 & 30360|WVE14696 & $8 / 28 / 08$ 10:37 & 291.4044 & 16 \\
\hline WVE14696-17 & \begin{tabular}{l|l|l|}
30582 & WVE14696
\end{tabular} & 9/3/08 17:02 & 291.4013 & 17 \\
\hline WVE14697-1 & 27733|WVE14697 & 7/11/08 18:32 & 293.2072 & 1 \\
\hline WVE14697-2 & \begin{tabular}{l|l|l}
27770 & WVE14697
\end{tabular} & 7/12/08 13:02 & 293.1938 & 2 \\
\hline WVE14697-3 & \begin{tabular}{|l|l|}
27808 & WVE14697 \\
\end{tabular} & $7 / 13 / 08$ 15:04 & 293.1904 & 3 \\
\hline WVE14697-4 & 27939|WVE14697 & $7 / 14 / 08$ 14:26 & 293.1948 & 4 \\
\hline WVE14697-5 & 27988 $\mid$ WVE14697 & 7/15/08 13:27| & 293.1884 & 5 \\
\hline WVE14697-6 & \begin{tabular}{l|l|l}
28024 & WVE14697
\end{tabular} & 7/15/08 19:21 & 293.1893 & 6 \\
\hline WVE14697-7 & \begin{tabular}{|l|l|}
28087 & WVE14697 \\
\end{tabular} & 7/16/08 19:26 & 293.1875 & 7 \\
\hline WVE14697-8 & 28121 $\mid$ WVE14697 & $7 / 17 / 08$ 14:44 & 293.1884 & 8 \\
\hline WVE14697-9 & 28265|WVE14697 & $7 / 18 / 08$ 15:32 & 293.1928 & 9 \\
\hline WVE14697-10 & 28398 $\mid$ WVE14697 & $7 / 21 / 08$ 11:23 & 293.1885 & 10 \\
\hline WVE14697-11 & 28746|WVE14697 & $7 / 25 / 08$ 13:27 & 293.1872 & 11 \\
\hline WVE14697-12 & \begin{tabular}{l|l|l}
29261 & WVE14697
\end{tabular} & $7 / 30 / 08$ 12:35 & 293.1979 & 12 \\
\hline WVE14697-13 & \begin{tabular}{l|l|l}
29660 & WVE14697
\end{tabular} & 8/7/08 19:36 & 293.1967 & 13 \\
\hline WVE14697-14 & \begin{tabular}{|l|l|}
29936 & WVE14697 \\
\end{tabular} & $8 / 14 / 08$ 16:47 & 293.2024 & 14 \\
\hline WVE14697-15 & \begin{tabular}{l|l|l}
30107 & WVE14697
\end{tabular} & $8 / 21 / 08$ 10:59 & 293.207 & 15 \\
\hline WVE14697-16 & \begin{tabular}{l|l|l|}
30361 & WVE14697 \\
\end{tabular} & 8/28/08 10:37 & 293.2121 & 16 \\
\hline WVE14697-17 & \begin{tabular}{l|l}
30583 & WVE14697 \\
\end{tabular} & 9/3/08 17:03 & 293.2154 & 17 \\
\hline
\end{tabular}




\begin{tabular}{|c|c|c|c|c|}
\hline WVE14698-1 & 27734 WVE14698 & 7/11/08 18:33 & 285.9307 & 1 \\
\hline WVE14698-2 & \begin{tabular}{l|l|l|}
27771 & WVE14698 \\
\end{tabular} & 7/12/08 13:02 & 285.932 & 2 \\
\hline WVE14698-3 & 27809 WVE14698 & 7/13/08 15:04 & 285.9292 & 3 \\
\hline WVE14698-4 & \begin{tabular}{|l|l|}
27940 & WVE14698 \\
\end{tabular} & 7/14/08 14:27 & 285.9473 & 4 \\
\hline WVE14698-5 & 27989|WVE14698 & 7/15/08 13:28 & 285.9428 & 5 \\
\hline WVE14698-6 & 28025|WVE14698 & 7/15/08 19:22 & 285.9439 & 6 \\
\hline WVE14698-7 & 28088 $\mid$ WVE14698 & 7/16/08 19:27| & 285.9351 & 7 \\
\hline WVE14698-8 & 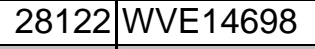 & 7/17/08 14:45 & 285.9448 & 8 \\
\hline WVE14698-9 & 28267|WVE14698 & 7/18/08 15:34 & 285.9431 & 9 \\
\hline WVE14698-10 & 28399 WVE14698 & 7/21/08 11:24 & 285.94 & 10 \\
\hline WVE14698-11 & 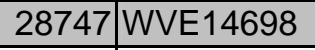 & 7/25/08 13:27 & 285.9424 & 11 \\
\hline WVE14698-12 & 29262 WVE14698 & 7/30/08 12:35 & 285.9589 & 12 \\
\hline WVE14698-13 & 29661 WVE14698 & 8/7/08 19:36 & 285.9567 & 13 \\
\hline WVE14698-14 & 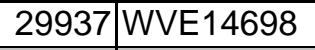 & 8/14/08 16:47 & 285.9651 & 14 \\
\hline WVE14698-15 & \begin{tabular}{|l|l|}
30108 & WVE14698 \\
\end{tabular} & 8/21/08 10:59 & 285.9713 & 15 \\
\hline WVE14698-16 & \begin{tabular}{|l|l|}
30362 & WVE14698 \\
\end{tabular} & 8/28/08 10:38 & 285.9773 & 16 \\
\hline WVE14698-17 & 30584|WVE14698 & 9/3/08 17:04 & 285.9668 & 17 \\
\hline WVE14699-1 & \begin{tabular}{l|l|l}
27735 & WVE14699 \\
\end{tabular} & 7/11/08 18:34 & 286.5916 & 1 \\
\hline WVE14699-2 & 27772 WVE14699 & 7/12/08 13:03 & 286.5835 & 2 \\
\hline WVE14699-3 & 27810|WVE14699 & 7/13/08 15:05 & 286.5777 & 3 \\
\hline WVE14699-4 & 27941 WVE14699 & $7 / 14 / 08$ 14:28 & 286.5765 & 4 \\
\hline WVE14699-5 & 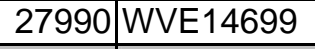 & 7/15/08 13:29 & 286.5728 & 5 \\
\hline WVE14699-6 & \begin{tabular}{|l|l|}
28026 & WVE14699 \\
\end{tabular} & 7/15/08 19:23 & 286.5726 & 6 \\
\hline WVE14699-7 & 28089 WVE14699 & 7/16/08 19:27 & 286.5718 & 7 \\
\hline WVE14699-8 & 28123 WVE14699 & 7/17/08 14:45 & 286.5702 & 8 \\
\hline WVE14699-9 & 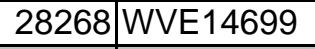 & 7/18/08 15:35 & 286.5772 & 9 \\
\hline WVE14699-10 & \begin{tabular}{|l|l|}
28400 & WVE14699 \\
\end{tabular} & 7/21/08 11:24 & 286.5754 & 10 \\
\hline WVE14699-11 & \begin{tabular}{|l|l|}
28748 & WVE14699 \\
\end{tabular} & $7 / 25 / 08$ 13:28 & 286.5802 & 11 \\
\hline WVE14699-12 & 29263 WVE14699 & $7 / 30 / 08$ 12:36 & 286.5893 & 12 \\
\hline WVE14699-13 & \begin{tabular}{l|l|l|}
29662 & WVE14699 \\
\end{tabular} & 8/7/08 19:37 & 286.5947 & 13 \\
\hline WVE14699-14 & \begin{tabular}{|l|l|}
29938 & WVE14699 \\
\end{tabular} & $8 / 14 / 08$ 16:48 & 286.602 & 14 \\
\hline WVE14699-15 & 30109 $\mid$ WVE14699 & $8 / 21 / 08$ 11:00 & 286.6001 & 15 \\
\hline WVE14699-16 & 30363 WVE14699 & $8 / 28 / 08$ 10:39 & 286.6116 & 16 \\
\hline WVE14699-17 & 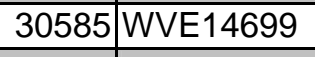 & 9/3/08 17:04 & 286.6167 & 17 \\
\hline WVE14700-1 & \begin{tabular}{|l|l|}
27736 & WVE14700 \\
\end{tabular} & 7/11/08 18:35 & 291.5435 & 1 \\
\hline WVE14700-2 & 27773 WVE14700 & 7/12/08 13:04 & 291.531 & 2 \\
\hline WVE14700-3 & 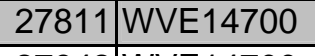 & 7/13/08 15:06 & 291.5333 & 3 \\
\hline WVE14700-4 & 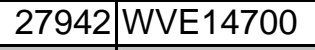 & $7 / 14 / 08$ 14:29 & 291.5349 & 4 \\
\hline WVE14700-5 & 27991 WVE14700 & 7/15/08 13:29| & 291.5264 & 5 \\
\hline WVE14700-6 & 28027 WVE14700 & 7/15/08 19:24 & 291.5326 & 6 \\
\hline WVE14700-7 & \begin{tabular}{|l|l|}
28090 & WVE14700 \\
\end{tabular} & 7/16/08 19:29 & 291.5287 & 7 \\
\hline WVE14700-8 & \begin{tabular}{l|l|l|}
28124 & WVE14700 \\
\end{tabular} & 7/17/08 14:46 & 291.5276 & 8 \\
\hline WVE14700-9 & 28269 WVE14700 & 7/18/08 15:36 & 291.5333 & 9 \\
\hline WVE14700-10 & 28401 WVE14700 & 7/21/08 11:25 & 291.5319 & 10 \\
\hline WVE14700-11 & 28749 WVE14700 & $7 / 25 / 08$ 13:29 & 291.5343 & 11 \\
\hline WVE14700-12 & \begin{tabular}{l|l|l|}
29264 & WVE14700 \\
\end{tabular} & 7/30/08 12:37 & 291.5419 & 12 \\
\hline WVE14700-13 & 29663 WVE14700 & 8/7/08 19:38 & 291.5462 & 13 \\
\hline WVE14700-14 & 29939 WVE14700 & $8 / 14 / 08$ 16:48 & 291.5495 & 14 \\
\hline WVE14700-15 & \begin{tabular}{l|l|l|}
30110 & WVE14700 \\
\end{tabular} & 8/21/08 11:01 & 291.5552 & 15 \\
\hline
\end{tabular}




\begin{tabular}{|c|c|c|c|c|}
\hline WVE14700-16 & \begin{tabular}{l|l|l}
30364 & WVE14700
\end{tabular} & 8/28/08 10:40 & 291.5622 & 16 \\
\hline WVE14700-17 & \begin{tabular}{l|l|}
30586 & WVE14700 \\
\end{tabular} & 9/3/08 17:06 & 291.567 & 17 \\
\hline WVE14821-1 & 28298 $\mid$ WVE14821 & 7/18/08 16:09 & 285.5108 & 1 \\
\hline WVE14821-2 & \begin{tabular}{l|l|}
28430 & WVE14821
\end{tabular} & 7/21/08 11:55 & 285.4954 & 2 \\
\hline WVE14821-3 & 28512 $\mid$ WVE14821 & $7 / 22 / 08$ 12:28 & 285.5078 & 3 \\
\hline WVE14821-4 & \begin{tabular}{|l|l|} 
28586 & WVE14821
\end{tabular} & 7/23/08 11:42 & 285.4965 & 4 \\
\hline WVE14821-5 & 28648 $\mid$ WVE14821 & $7 / 24 / 08$ 12:20 & 285.4928 & 5 \\
\hline WVE14821-6 & \begin{tabular}{l|l|}
28692 & WVE14821
\end{tabular} & 7/24/08 18:11 & 285.4884 & 6 \\
\hline WVE14821-7 & \begin{tabular}{|l|l|}
28723 & WVE14821 \\
\end{tabular} & 7/25/08 13:09 & 285.4881 & 7 \\
\hline WVE14821-8 & \begin{tabular}{|l|l|}
28910 & $W V E 14821$
\end{tabular} & 7/26/08 14:59 & 285.487 & 8 \\
\hline WVE14821-9 & \begin{tabular}{|l|l|}
29702 & WVE14821
\end{tabular} & 8/7/08 20:07 & 285.4895 & 9 \\
\hline WVE14821-10 & \begin{tabular}{l|l|}
29902 & WVE14821 \\
\end{tabular} & $8 / 14 / 08$ 16:24 & 285.4924 & 10 \\
\hline WVE14821-11 & 30073|WVE14821 & $8 / 21 / 08$ 10:33 & 285.4896 & 11 \\
\hline WVE14821-12 & 30328 $\mid$ WVE14821 & 8/28/08 10:09 & 285.4933 & 12 \\
\hline WVE14821-13 & 30545|WVE14821 & 9/3/08 15:22 & 285.4976 & 13 \\
\hline WVE14822-1 & $28299 \mid$ WVE14822 & 7/18/08 16:09 & 282.6592 & 1 \\
\hline WVE14822-2 & 28431|WVE14822 & $7 / 21 / 08$ 11:56 & 282.6526 & 2 \\
\hline WVE14822-3 & 28513|WVE14822 & $7 / 22 / 08$ 12:29 & 282.6675 & 3 \\
\hline WVE14822-4 & 28587 $\mid$ WVE14822 & $7 / 23 / 08$ 11:43 & 282.6557 & 4 \\
\hline WVE14822-5 & \begin{tabular}{|l|l|}
28936 & WVE14822 \\
\end{tabular} & 7/26/08 15:31 & 282.5968 & 5 \\
\hline WVE14822-6 & 29004|WVE14822 & 7/27/08 14:39 & 282.5972 & 6 \\
\hline WVE14822-7 & 29059 $\mid$ WVE14822 & $7 / 28 / 08$ 18:36 & 282.5945 & 7 \\
\hline WVE14822-8 & 29127 $\mid$ WVE14822 & $7 / 29 / 08$ 12:38 & 282.5934 & 8 \\
\hline WVE14822-9 & 29297|WVE14822 & 7/31/08 15:30 & 281.7015 & 9 \\
\hline WVE14822-10 & 29321 |WVE14822 & 8/1/08 13:48 & 281.704 & 10 \\
\hline WVE14822-11 & 29390|WVE14822 & $8 / 2 / 0814: 36$ & 281.7148 & 11 \\
\hline WVE14822-12 & 29438|WVE14822 & $8 / 3 / 08$ 12:43 & 281.7215 & 12 \\
\hline WVE14822-13 & 29715|WVE14822 & 8/7/08 20:16 & 281.7414 & 13 \\
\hline WVE14822-14 & 29846|WVE14822 & $8 / 14 / 08$ 15:41 & 281.7667 & 14 \\
\hline WVE14822-15 & \begin{tabular}{|l|l|}
30016 & WVE14822
\end{tabular} & 8/21/08 9:49 & 281.7838 & 15 \\
\hline WVE14822-16 & $30271 \mid$ WVE14822 & 8/28/08 9:25 & 281.7927 & 16 \\
\hline WVE14822-17 & \begin{tabular}{l|l|l|}
30492 & WVE14822
\end{tabular} & 9/3/08 14:30 & 281.8031 & 17 \\
\hline WVE14823-1 & 28300 WVE14823 & 7/18/08 16:10 & 286.0018 & 1 \\
\hline WVE14823-2 & \begin{tabular}{|l|l|}
28432 & WVE14823
\end{tabular} & $7 / 21 / 08$ 11:56 & 285.9879 & 2 \\
\hline WVE14823-3 & \begin{tabular}{|l|l|}
28514 & WVE14823 \\
\end{tabular} & $7 / 22 / 08$ 12:30 & 285.9876 & 3 \\
\hline WVE14823-4 & \begin{tabular}{l|l|}
28588 & WVE14823
\end{tabular} & 7/23/08 11:43 & 285.9876 & 4 \\
\hline WVE14823-5 & 28929|WVE14823 & $7 / 26 / 08$ 15:25 & 285.9405 & 5 \\
\hline WVE14823-6 & 29001 WVE14823 & 7/27/08 14:37 & 285.9395 & 6 \\
\hline WVE14823-7 & \begin{tabular}{|l|l|}
29056 & WVE14823 \\
\end{tabular} & $7 / 28 / 08$ 18:34 & 285.9367 & 7 \\
\hline WVE14823-8 & 29124 $\mid$ WVE14823 & $7 / 29 / 08$ 12:36 & 285.9389 & 8 \\
\hline WVE14823-9 & 29298|WVE14823 & $7 / 31 / 08$ 15:31 & 285.2066 & 9 \\
\hline WVE14823-10 & \begin{tabular}{l|l|}
29322 & WVE14823
\end{tabular} & 8/1/08 13:49 & 285.2166 & 10 \\
\hline WVE14823-11 & 29391|WVE14823 & $8 / 2 / 0814: 37$ & 285.2241 & 11 \\
\hline WVE14823-12 & \begin{tabular}{|l|l|}
29437 & WVE14823 \\
\end{tabular} & $8 / 3 / 08$ 12:42 & 285.2315 & 12 \\
\hline WVE14823-13 & $29714 \mid$ WVE14823 & 8/7/08 20:15 & 285.2526 & 13 \\
\hline WVE14823-14 & \begin{tabular}{|l|l|}
29851 & WVE14823 \\
\end{tabular} & $8 / 14 / 08$ 15:44 & 285.2948 & 14 \\
\hline WVE14823-15 & $30021 \mid$ WVE14823 & 8/21/08 9:52 & 285.3174 & 15 \\
\hline WVE14823-16 & \begin{tabular}{|l|l|}
30276 & WVE14823 \\
\end{tabular} & 8/28/08 9:29 & 285.3478 & 16 \\
\hline WVE14823-17 & \begin{tabular}{l|l|l|}
30497 & WVE14823 \\
\end{tabular} & 9/3/08 14:34 & 285.367 & 17 \\
\hline
\end{tabular}




\begin{tabular}{|c|c|c|c|c|}
\hline WVE14824-1 & 28301|WVE14824 & 7/18/08 16:11 & 288.7015 & 1 \\
\hline WVE14824-2 & \begin{tabular}{l|l|} 
28433 & WVE14824
\end{tabular} & $7 / 21 / 08$ 11:57 & 288.69 & 2 \\
\hline WVE14824-3 & \begin{tabular}{l|l|}
28515 & WVE14824
\end{tabular} & $7 / 22 / 08$ 12:31 & 288.6886 & 3 \\
\hline WVE14824-4 & \begin{tabular}{l|l|}
28589 & WVE14824
\end{tabular} & $7 / 23 / 08$ 11:44 & 288.6898 & 4 \\
\hline WVE14824-5 & \begin{tabular}{|l|l|}
28928 & WVE14824 \\
\end{tabular} & $7 / 26 / 08$ 15:24 & 288.6514 & 5 \\
\hline WVE14824-6 & 29002 $\mid$ WVE14824 & 7/27/08 14:37 & 288.6541 & 6 \\
\hline WVE14824-7 & \begin{tabular}{|l|l|}
29057 & WV14824
\end{tabular} & $7 / 28 / 08$ 18:34 & 288.6469 & 7 \\
\hline WVE14824-8 & \begin{tabular}{l|l|l|}
29125 & WVE14824 \\
\end{tabular} & $7 / 29 / 08$ 12:36 & 288.6502 & 8 \\
\hline WVE14824-9 & \begin{tabular}{|l|l|}
29299 & WVE14824 \\
\end{tabular} & $7 / 31 / 08$ 15:33 & 287.8741 & 9 \\
\hline WVE14824-10 & \begin{tabular}{|l|l|}
29323 & WVE14824 \\
\end{tabular} & $8 / 1 / 08$ 13:50 & 287.8865 & 10 \\
\hline WVE14824-11 & \begin{tabular}{l|l|} 
29392 & WVE14824 \\
\end{tabular} & $8 / 2 / 08$ 14:38 & 287.8977 & 11 \\
\hline WVE14824-12 & \begin{tabular}{l|l|l|}
29436 & WVE14824
\end{tabular} & $8 / 3 / 08$ 12:42 & 287.9013 & 12 \\
\hline WVE14824-13 & \begin{tabular}{l|l|l} 
29710 & WVE14824
\end{tabular} & 8/7/08 20:12 & 287.9209 & 13 \\
\hline WVE14824-14 & \begin{tabular}{l|l|}
29852 & WVE14824
\end{tabular} & $8 / 14 / 08$ 15:45 & 287.9416 & 14 \\
\hline WVE14824-15 & \begin{tabular}{l|l|}
30022 & WVE14824 \\
\end{tabular} & 8/21/08 9:53 & 287.9751 & 15 \\
\hline WVE14824-16 & \begin{tabular}{l|l|}
30277 & WVE14824
\end{tabular} & 8/28/08 9:30 & 288.0164 & 16 \\
\hline WVE14824-17 & \begin{tabular}{|l|l|}
30498 & WVE14824 \\
\end{tabular} & 9/3/08 14:35 & 288.0443 & 17 \\
\hline WVE14825-1 & \begin{tabular}{l|l|l|}
28302 & WVE14825 \\
\end{tabular} & 7/18/08 16:12 & 287.7571 & 1 \\
\hline WVE14825-2 & \begin{tabular}{l|l|l}
28434 & WVE14825 \\
\end{tabular} & $7 / 21 / 08$ 11:58 & 287.7454 & 2 \\
\hline WVE14825-3 & \begin{tabular}{|l|l|}
28516 & $W V 14825$
\end{tabular} & $7 / 22 / 08$ 12:32 & 287.7444 & 3 \\
\hline WVE14825-4 & \begin{tabular}{l|l|l}
28590 & WVE14825 \\
\end{tabular} & $7 / 23 / 08$ 11:45 & 287.7468 & 4 \\
\hline WVE14825-5 & \begin{tabular}{l|l|l|}
28931 & WVE14825 \\
\end{tabular} & $7 / 26 / 08$ 15:27 & 287.6959 & 5 \\
\hline WVE14825-6 & 28999 WVE14825 & $7 / 27 / 08$ 14:35 & 287.6942 & 6 \\
\hline WVE14825-7 & \begin{tabular}{l|l|l|}
29054 & WVE14825
\end{tabular} & 7/28/08 18:32 & 287.6903 & 7 \\
\hline WVE14825-8 & \begin{tabular}{l|l|l}
29122 & WVE14825
\end{tabular} & $7 / 29 / 08$ 12:34 & 287.6949 & 8 \\
\hline WVE14825-9 & \begin{tabular}{l|l|l}
29300 & WVE14825 \\
\end{tabular} & 7/31/08 15:34 & 286.9288 & 9 \\
\hline WVE14825-10 & \begin{tabular}{l|l|l|}
29324 & WVE14825
\end{tabular} & 8/1/08 13:51 & 286.9369 & 10 \\
\hline WVE14825-11 & 29393|WVE14825 & $8 / 2 / 08$ 14:39 & 286.9514 & 11 \\
\hline WVE14825-12 & \begin{tabular}{l|l|l|}
29435 & WVE14825
\end{tabular} & $8 / 3 / 08$ 12:41 & 286.9579 & 12 \\
\hline WVE14825-13 & \begin{tabular}{l|l|l|}
29711 & WVE14825 \\
\end{tabular} & 8/7/08 20:13 & 286.9796 & 13 \\
\hline WVE14825-14 & 29853 WVE14825 & $8 / 14 / 08$ 15:46 & 286.9917 & 14 \\
\hline WVE14825-15 & 30023|WVE14825 & 8/21/08 9:54 & 287.0235 & 15 \\
\hline WVE14825-16 & 30278 WVE14825 & 8/28/08 9:31 & 287.0526 & 16 \\
\hline WVE14825-17 & 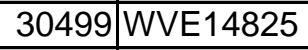 & 9/3/08 14:36 & 287.0833 & 17 \\
\hline WVE14826-1 & 28303 WVE14826 & $7 / 18 / 08$ 16:13 & 284.5549 & 1 \\
\hline WVE14826-2 & $28435 \mid$ WVE14826 & $7 / 21 / 08$ 11:59 & 284.5281 & 2 \\
\hline WVE14826-3 & \begin{tabular}{l|l|l|}
28517 & WVE14826 \\
\end{tabular} & $7 / 22 / 08$ 12:33 & 284.5424 & 3 \\
\hline WVE14826-4 & \begin{tabular}{l|l|l|}
28591 & WVE14826 \\
\end{tabular} & $7 / 23 / 08$ 11:46 & 284.5277 & 4 \\
\hline WVE14826-5 & 28933 WVE14826 & $7 / 26 / 08$ 15:28 & 284.4806 & 5 \\
\hline WVE14826-6 & \begin{tabular}{l|l|l|}
29007 & WVE14826
\end{tabular} & $7 / 27 / 08$ 14:40 & 284.4852 & 6 \\
\hline WVE14826-7 & \begin{tabular}{l|l|}
29062 & WVE14826 \\
\end{tabular} & 7/28/08 18:38 & 284.4858 & 7 \\
\hline WVE14826-8 & \begin{tabular}{|l|l|}
29130 & WVE14826 \\
\end{tabular} & 7/29/08 12:39 & 284.4805 & 8 \\
\hline WVE14826-9 & \begin{tabular}{|l|l|}
29301 & WVE14826 \\
\end{tabular} & 7/31/08 15:34 & 283.7075 & 9 \\
\hline WVE14826-10 & 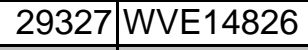 & 8/1/08 13:54 & 283.7148 & 10 \\
\hline WVE14826-11 & \begin{tabular}{|l|l|}
29396 & WVE14826 \\
\end{tabular} & $8 / 2 / 08$ 14:43 & 283.7386 & 11 \\
\hline WVE14826-12 & \begin{tabular}{l|l|l|}
29442 & WVE14826 \\
\end{tabular} & $8 / 3 / 08$ 12:46 & 283.747 & 12 \\
\hline WVE14826-13 & \begin{tabular}{l|l|l|}
29717 & WVE14826 \\
\end{tabular} & 8/7/08 20:17 & 283.7699 & 13 \\
\hline WVE14826-14 & 29854 WVE14826 & $8 / 14 / 08$ 15:46 & 283.7972 & 14 \\
\hline WVE14826-15 & \begin{tabular}{|l|l|}
30024 & WVE14826 \\
\end{tabular} & 8/21/08 9:55 & 283.8273 & 15 \\
\hline
\end{tabular}




\begin{tabular}{|c|c|c|c|c|}
\hline WVE14826-16 & \begin{tabular}{l|l|l|}
30279 & WVE14826
\end{tabular} & 8/28/08 9:32 & 283.8596 & 16 \\
\hline WVE14826-17 & $30500 \mid$ WVE14826 & 9/3/08 14:36 & 283.8884 & 17 \\
\hline WVE14827-1 & 28304|WVE14827 & $7 / 18 / 08$ 16:13 & 285.434 & 1 \\
\hline WVE14827-2 & \begin{tabular}{l|l|} 
28436 & WVE14827
\end{tabular} & 7/21/08 11:59 & 285.4239 & 2 \\
\hline WVE14827-3 & 28518|WVE14827 & $7 / 22 / 08$ 12:33 & 285.4251 & 3 \\
\hline WVE14827-4 & \begin{tabular}{l|l|}
28592 & WVE14827
\end{tabular} & 7/23/08 11:46 & 285.4237 & 4 \\
\hline WVE14827-5 & 28934|WVE14827 & $7 / 26 / 08$ 15:29 & 285.3871 & 5 \\
\hline WVE14827-6 & \begin{tabular}{l|l|}
29006 & WVE14827
\end{tabular} & 7/27/08 14:40 & 285.3829 & 6 \\
\hline WVE14827-7 & \begin{tabular}{|l|l|}
29061 & WVE14827 \\
\end{tabular} & 7/28/08 18:37 & 285.3807 & 7 \\
\hline WVE14827-8 & \begin{tabular}{|l|l|}
29129 & WVE14827
\end{tabular} & 7/29/08 12:39 & 285.3807 & 8 \\
\hline WVE14827-9 & \begin{tabular}{|l|l|}
29302 & WVE14827
\end{tabular} & 7/31/08 15:35 & 284.6726 & 9 \\
\hline WVE14827-10 & \begin{tabular}{|l|l|} 
29328 & WVE14827
\end{tabular} & 8/1/08 13:55 & 284.6889 & 10 \\
\hline WVE14827-11 & 29397 $\mid$ WVE14827 & $8 / 2 / 0814: 43$ & 284.6976 & 11 \\
\hline WVE14827-12 & \begin{tabular}{|l|l|}
29443 & WVE14827
\end{tabular} & $8 / 3 / 08$ 12:47 & 284.704 & 12 \\
\hline WVE14827-13 & 29718|WVE14827 & 8/7/08 20:18 & 284.7256 & 13 \\
\hline WVE14827-14 & \begin{tabular}{l|l|}
29855 & WVE14827
\end{tabular} & $8 / 14 / 08$ 15:47 & 284.7671 & 14 \\
\hline WVE14827-15 & $30025 \mid$ WVE14827 & 8/21/08 9:55 & 284.809 & 15 \\
\hline WVE14827-16 & 30280|WVE14827 & 8/28/08 9:32 & 284.846 & 16 \\
\hline WVE14827-17 & $30501 \mid$ WVE14827 & 9/3/08 14:37 & 284.8668 & 17 \\
\hline WVE14828-1 & \begin{tabular}{|l|l|}
28305 & WVE14828 \\
\end{tabular} & 7/18/08 16:14 & 284.7373 & 1 \\
\hline WVE14828-2 & \begin{tabular}{|l|l|l|}
28437 & WVE14828
\end{tabular} & $7 / 21 / 08$ 12:00 & 284.7231 & 2 \\
\hline WVE14828-3 & 28519 WVE14828 & $7 / 22 / 08$ 12:35 & 284.7227 & 3 \\
\hline WVE14828-4 & 28593|WVE14828 & $7 / 23 / 08$ 11:47| & 284.7209 & 4 \\
\hline WVE14828-5 & \begin{tabular}{l|l|l|}
28657 & WVE14828
\end{tabular} & 7/24/08 12:28 & 284.7021 & 5 \\
\hline WVE14828-6 & 28702|WVE14828 & 7/24/08 18:18 & 284.6989 & 6 \\
\hline WVE14828-7 & 28737 $\mid$ WVE14828 & $7 / 25 / 08$ 13:20 & 284.6987 & 7 \\
\hline WVE14828-8 & 28919|WVE14828 & $7 / 26 / 08$ 15:05 & 284.7042 & 8 \\
\hline WVE14828-9 & 29333|WVE14828 & 8/1/08 14:00 & 283.9405 & 9 \\
\hline WVE14828-10 & 29402 $\mid$ WVE14828 & 8/2/08 14:48 & 283.9496 & 10 \\
\hline WVE14828-11 & 29448|WVE14828 & 8/3/08 12:51 & 283.9542 & 11 \\
\hline WVE14828-12 & \begin{tabular}{|l|l|}
29472 & WVE14828 \\
\end{tabular} & 8/4/08 13:04 & 283.9641 & 12 \\
\hline WVE14828-13 & 29723|WVE14828 & 8/7/08 20:22 & 283.9799 & 13 \\
\hline WVE14828-14 & 29882 $\mid$ WVE14828 & 8/14/08 16:06 & 284.0111 & 14 \\
\hline WVE14828-15 & \begin{tabular}{l|l|l|}
30052 & WVE14828
\end{tabular} & $8 / 21 / 08$ 10:15 & 284.0425 & 15 \\
\hline WVE14828-16 & \begin{tabular}{|l|l|}
30307 & WVE14828 \\
\end{tabular} & 8/28/08 9:52 & 284.0723 & 16 \\
\hline WVE14828-17 & 30528 $\mid$ WVE14828 & 9/3/08 15:06 & 284.0982 & 17 \\
\hline WVE14829-1 & 28308 $\mid$ WVE14829 & 7/18/08 16:18 & 286.1398 & 1 \\
\hline WVE14829-2 & \begin{tabular}{|l|l|l|}
28440 & WVE14829
\end{tabular} & $7 / 21 / 08$ 12:03 & 286.1316 & 2 \\
\hline WVE14829-3 & \begin{tabular}{l|l|}
28522 & WVE14829 \\
\end{tabular} & $7 / 22 / 08$ 12:38 & 286.1288 & 3 \\
\hline WVE14829-4 & \begin{tabular}{|l|l|}
28596 & WVE14829 \\
\end{tabular} & $7 / 23 / 08$ 11:50 & 286.1262 & 4 \\
\hline WVE14829-5 & 28658|WVE14829 & $7 / 24 / 08$ 12:29 & 286.1224 & 5 \\
\hline WVE14829-6 & 28703 WVE14829 & $7 / 24 / 08$ 18:19 & 286.1166 & 6 \\
\hline WVE14829-7 & 28732 $\mid$ WVE14829 & $7 / 25 / 08$ 13:16 & 286.1134 & 7 \\
\hline WVE14829-8 & \begin{tabular}{|l|l|} 
28918 & WVE14829 \\
\end{tabular} & 7/26/08 15:04 & 286.1128 & 8 \\
\hline WVE14829-9 & 29334 $\mid$ WVE14829 & $8 / 1 / 08$ 14:00 & 285.354 & 9 \\
\hline WVE14829-10 & \begin{tabular}{|l|l|}
29403 & WVE14829 \\
\end{tabular} & $8 / 2 / 08$ 14:49 & 285.3669 & 10 \\
\hline WVE14829-11 & $29449 \mid$ WVE14829 & 8/3/08 12:52 & 285.3745 & 11 \\
\hline WVE14829-12 & 29473 WVE14829 & 8/4/08 13:05 & 285.3841 & 12 \\
\hline WVE14829-13 & \begin{tabular}{|l|l|}
29726 & WVE14829 \\
\end{tabular} & 8/7/08 20:23 & 285.3977 & 13 \\
\hline
\end{tabular}




\begin{tabular}{|c|c|c|c|c|}
\hline WVE14829-14 & 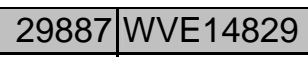 & 8/14/08 16:11 & 285.432 & 14 \\
\hline WVE14829-15 & \begin{tabular}{l|l|l|}
30057 & WVE14829 \\
\end{tabular} & 8/21/08 10:19 & 285.4539 & 15 \\
\hline WVE14829-16 & \begin{tabular}{|l|l|}
30312 & WVE14829
\end{tabular} & $8 / 28 / 08$ 9:56 & 285.4739 & 16 \\
\hline WVE14829-17 & \begin{tabular}{l|l}
30533 & WVE14829
\end{tabular} & 9/3/08 15:10 & 285.4922 & 17 \\
\hline WVE14830-1 & \begin{tabular}{|l|l|l}
28309 & WVE14830
\end{tabular} & 7/18/08 16:18 & 286.4115 & 1 \\
\hline WVE14830-2 & 28441 $\mid$ WVE14830 & $7 / 21 / 08$ 12:04 & 286.3966 & 2 \\
\hline WVE14830-3 & 28523 WVE14830 & $7 / 22 / 08$ 12:39 & 286.3957 & 3 \\
\hline WVE14830-4 & \begin{tabular}{l|l|l|}
28597 & WVE14830 \\
\end{tabular} & $7 / 23 / 08$ 11:51 & 286.3977 & 4 \\
\hline WVE14830-5 & 28659 WVE14830 & $7 / 24 / 08$ 12:29 & 286.3909 & 5 \\
\hline WVE14830-6 & \begin{tabular}{l|l|l|}
28704 & WVE14830 \\
\end{tabular} & $7 / 24 / 08$ 18:20 & 286.3863 & 6 \\
\hline WVE14830-7 & 28733 WVE14830 & 7/25/08 13:17 & 286.3831 & 7 \\
\hline WVE14830-8 & $28915 \mid$ WVE14830 & $7 / 26 / 08$ 15:03 & 286.3864 & 8 \\
\hline WVE14830-9 & 29335 WVE14830 & 8/1/08 14:02 & 285.6142 & 9 \\
\hline WVE14830-10 & 29404 $\mid$ WVE14830 & $8 / 2 / 08$ 14:50 & 285.6358 & 10 \\
\hline WVE14830-11 & \begin{tabular}{l|l|l|}
29454 & WVE14830 \\
\end{tabular} & $8 / 3 / 08$ 12:56 & 285.6483 & 11 \\
\hline WVE14830-12 & 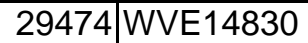 & $8 / 4 / 08$ 13:05 & 285.6538 & 12 \\
\hline WVE14830-13 & 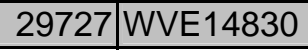 & 8/7/08 20:24 & 285.6781 & 13 \\
\hline WVE14830-14 & \begin{tabular}{|l|l|}
29888 & WVE14830 \\
\end{tabular} & 8/14/08 16:11 & 285.7055 & 14 \\
\hline WVE14830-15 & \begin{tabular}{|l|l|}
30058 & WVE14830 \\
\end{tabular} & $8 / 21 / 08$ 10:21 & 285.7342 & 15 \\
\hline WVE14830-16 & 30313|WVE14830 & 8/28/08 9:57 & 285.7717 & 16 \\
\hline WVE14830-17 & \begin{tabular}{l|l|l|}
30534 & WVE14830 \\
\end{tabular} & 9/3/08 15:11 & 285.7995 & 17 \\
\hline WVE14876-1 & \begin{tabular}{|l|l|}
28310 & WVE14876 \\
\end{tabular} & 7/18/08 16:19 & 287.5192 & 1 \\
\hline WVE14876-2 & \begin{tabular}{l|l|l|}
28442 & WVE14876 \\
\end{tabular} & $7 / 21 / 08$ 12:05 & 287.5203 & 2 \\
\hline WVE14876-3 & $28524 \mid$ WVE14876 & $7 / 22 / 08$ 12:40 & 287.52 & 3 \\
\hline WVE14876-4 & 28598 $\mid$ WVE14876 & $7 / 23 / 08$ 11:51 & 287.5223 & 4 \\
\hline WVE14876-5 & 28649 WVE14876 & $7 / 24 / 08$ 12:21 & 287.5194 & 5 \\
\hline WVE14876-6 & \begin{tabular}{l|l|l|}
28693 & WVE14876
\end{tabular} & $7 / 24 / 08$ 18:12 & 287.5187 & 6 \\
\hline WVE14876-7 & $28724 \mid$ WVE14876 & 7/25/08 13:10 & 287.5169 & 7 \\
\hline WVE14876-8 & 28909 WVE14876 & $7 / 26 / 08$ 14:59 & 287.5206 & 8 \\
\hline WVE14876-9 & \begin{tabular}{l|l|l|}
29703 & WVE14876 \\
\end{tabular} & 8/7/08 20:07 & 287.5285 & 9 \\
\hline WVE14876-10 & 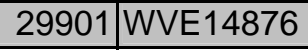 & 8/14/08 16:23 & 287.5287 & 10 \\
\hline WVE14876-11 & \begin{tabular}{l|l|l|}
30072 & WVE14876 \\
\end{tabular} & $8 / 21 / 08$ 10:32 & 287.5265 & 11 \\
\hline WVE14876-12 & 30327 WVE14876 & 8/28/08 10:08 & 287.5327 & 12 \\
\hline WVE14876-13 & \begin{tabular}{l|l|l|}
30546 & WVE14876 \\
\end{tabular} & 9/3/08 15:23 & 287.5359 & 13 \\
\hline WVE14877-1 & \begin{tabular}{l|l|l}
28311 & WVE14877 \\
\end{tabular} & 7/18/08 16:20 & 287.7626 & 1 \\
\hline WVE14877-2 & \begin{tabular}{l|l|l|}
28443 & WVE14877
\end{tabular} & $7 / 21 / 08$ 12:06 & 287.7617 & 2 \\
\hline WVE14877-3 & \begin{tabular}{l|l|l}
28525 & WVE14877 \\
\end{tabular} & $7 / 22 / 08$ 12:41 & 287.7632 & 3 \\
\hline WVE14877-4 & \begin{tabular}{l|l|l}
28599 & WVE14877
\end{tabular} & $7 / 23 / 08$ 11:52 & 287.7623 & 4 \\
\hline WVE14877-5 & \begin{tabular}{l|l|l|}
28932 & WVE14877 \\
\end{tabular} & 7/26/08 15:27 & 287.7059 & 5 \\
\hline WVE14877-6 & 28998 $\mid$ WVE14877 & $7 / 27 / 08$ 14:35 & 287.7025 & 6 \\
\hline WVE14877-7 & 29053 WVE14877 & 7/28/08 18:32 & 287.6983 & 7 \\
\hline WVE14877-8 & \begin{tabular}{|l|l|}
29121 & WVE14877 \\
\end{tabular} & 7/29/08 12:34 & 287.7023 & 8 \\
\hline WVE14877-9 & \begin{tabular}{|l|l|}
29693 & WVE14877 \\
\end{tabular} & 8/7/08 20:01 & 287.7036 & 9 \\
\hline WVE14877-10 & \begin{tabular}{l|l|l|}
29861 & WVE14877 \\
\end{tabular} & 8/14/08 15:51 & 287.7018 & 10 \\
\hline WVE14877-11 & \begin{tabular}{|l|l|}
30031 & WVE14877 \\
\end{tabular} & 8/21/08 9:59 & 287.7033 & 11 \\
\hline WVE14877-12 & \begin{tabular}{|l|l|}
30286 & WVE14877 \\
\end{tabular} & 8/28/08 9:36 & 287.7079 & 12 \\
\hline WVE14877-13 & \begin{tabular}{l|l|l}
30507 & WVE14877 \\
\end{tabular} & 9/3/08 14:42 & 287.709 & 13 \\
\hline WVE14878-1 & \begin{tabular}{l|l|l|}
28312 & WVE14878 \\
\end{tabular} & 7/18/08 16:21 & 284.4293 & 1 \\
\hline WVE14878-2 & \begin{tabular}{l|l|l|}
28444 & WVE14878 \\
\end{tabular} & 7/21/08 12:07 & 284.4246 & 2 \\
\hline
\end{tabular}




\begin{tabular}{|c|c|c|c|c|}
\hline WVE14878-3 & \begin{tabular}{l|l|l}
28526 & WVE14878 \\
\end{tabular} & $7 / 22 / 08$ 12:42 & 284.4237 & 3 \\
\hline WVE14878-4 & 28600 $\mid$ WVE14878 & 7/23/08 11:53 & 284.4242 & 4 \\
\hline WVE14878-5 & $28935 \mid$ WVE14878 & $7 / 26 / 08$ 15:30 & 284.3833 & 5 \\
\hline WVE14878-6 & \begin{tabular}{l|l|l|}
29005 & WVE14878
\end{tabular} & 7/27/08 14:39 & 284.3828 & 6 \\
\hline WVE14878-7 & 29060|WVE14878 & $7 / 28 / 08$ 18:36 & 284.3787 & 7 \\
\hline WVE14878-8 & 29128 $\mid$ WVE14878 & 7/29/08 12:38 & 284.3798 & 8 \\
\hline WVE14878-9 & 29377|WVE14878 & $8 / 2 / 08$ 14:25 & 283.5463 & 9 \\
\hline WVE14878-10 & 29420|WVE14878 & 8/3/08 12:30 & 283.5564 & 10 \\
\hline WVE14878-11 & \begin{tabular}{|l|l|}
29480 & WVE14878 \\
\end{tabular} & 8/4/08 13:16 & 283.568 & 11 \\
\hline WVE14878-12 & $29515 \mid$ WVE14878 & 8/5/08 13:00 & 283.5677 & 12 \\
\hline WVE14878-13 & 29737|WVE14878 & 8/7/08 20:36 & 283.5851 & 13 \\
\hline WVE14878-14 & 29870 WVE14878 & $8 / 14 / 08$ 15:58 & 283.6084 & 14 \\
\hline WVE14878-15 & 30044 WVE14878 & $8 / 21 / 08$ 10:09 & 283.6267 & 15 \\
\hline WVE14878-16 & 30295|WVE14878 & 8/28/08 9:43 & 283.6781 & 16 \\
\hline WVE14878-17 & 30516|WVE14878 & 9/3/08 14:53 & 283.6961 & 17 \\
\hline WVE14879-1 & \begin{tabular}{l|l|} 
28313 & WVE14879
\end{tabular} & 7/18/08 16:22 & 283.0535 & 1 \\
\hline WVE14879-2 & 28445|WVE14879 & $7 / 21 / 08$ 12:07 & 283.0574 & 2 \\
\hline WVE14879-3 & 28527|WVE14879 & $7 / 22 / 08$ 12:43 & 283.056 & 3 \\
\hline WVE14879-4 & $28601 \mid$ WVE14879 & $7 / 23 / 08$ 11:53 & 283.0586 & 4 \\
\hline WVE14879-5 & \begin{tabular}{l|l|}
28942 & WVE14879 \\
\end{tabular} & 7/26/08 15:35 & 283.0174 & 5 \\
\hline WVE14879-6 & $28989 \mid$ WVE14879 & 7/27/08 14:27| & 283.015 & 6 \\
\hline WVE14879-7 & \begin{tabular}{|l|l|}
29043 & WVE14879 \\
\end{tabular} & $7 / 28 / 08$ 18:25 & 283.0134 & 7 \\
\hline WVE14879-8 & 29111 $\mid$ WVE14879 & $7 / 29 / 08$ 12:27| & 283.0125 & 8 \\
\hline WVE14879-9 & \begin{tabular}{l|l}
29378 & WVE14879
\end{tabular} & $8 / 2 / 0814: 26$ & 282.1508 & 9 \\
\hline WVE14879-10 & 29423|WVE14879 & 8/3/08 12:32 & 282.161 & 10 \\
\hline WVE14879-11 & 29481 $\mid$ WVE14879 & 8/4/08 13:16 & 282.1757 & 11 \\
\hline WVE14879-12 & 29516|WVE14879 & 8/5/08 13:01 & 282.1791 & 12 \\
\hline WVE14879-13 & \begin{tabular}{l|l|}
29736 & WVE14879
\end{tabular} & 8/7/08 20:35 & 282.1905 & 13 \\
\hline WVE14879-14 & 29875|WVE14879 & $8 / 14 / 08$ 16:02 & 282.2176 & 14 \\
\hline WVE14879-15 & \begin{tabular}{l|l|l|}
30045 & WVE14879
\end{tabular} & 8/21/08 10:10 & 282.2513 & 15 \\
\hline WVE14879-16 & \begin{tabular}{|l|l|}
30300 & WVE14879 \\
\end{tabular} & 8/28/08 9:47 & 282.3142 & 16 \\
\hline WVE14879-17 & 30521 $\mid$ WVE14879 & 9/3/08 14:58 & 282.3437 & 17 \\
\hline WVE14880-1 & 28314 $\mid$ WVE14880 & 7/18/08 16:22 & 282.6169 & 1 \\
\hline WVE14880-2 & \begin{tabular}{|l|l|}
28446 & WVE14880
\end{tabular} & $7 / 21 / 08$ 12:08 & 282.6212 & 2 \\
\hline WVE14880-3 & \begin{tabular}{|l|l|}
28528 & WVE14880 \\
\end{tabular} & $7 / 22 / 08$ 12:44 & 282.6227 & 3 \\
\hline WVE14880-4 & \begin{tabular}{l|l|}
28602 & WVE14880 \\
\end{tabular} & 7/23/08 11:54 & 282.6232 & 4 \\
\hline WVE14880-5 & 28941 $\mid$ WVE14880 & $7 / 26 / 08$ 15:34 & 282.5937 & 5 \\
\hline WVE14880-6 & 28990 WVE14880 & $7 / 27 / 08$ 14:28 & 282.5891 & 6 \\
\hline WVE14880-7 & \begin{tabular}{|l|l|}
29044 & WVE14880 \\
\end{tabular} & $7 / 28 / 08$ 18:25 & 282.5848 & 7 \\
\hline WVE14880-8 & \begin{tabular}{|l|l|l|}
29112 & WVE14880
\end{tabular} & $7 / 29 / 08$ 12:27 & 282.5903 & 8 \\
\hline WVE14880-9 & 29379 WVE14880 & 8/2/08 14:27 & 281.7459 & 9 \\
\hline WVE14880-10 & \begin{tabular}{l|l|l|}
29424 & WVE14880
\end{tabular} & 8/3/08 12:33 & 281.7643 & 10 \\
\hline WVE14880-11 & \begin{tabular}{l|l|}
29482 & WVE14880
\end{tabular} & 8/4/08 13:17 & 281.7797 & 11 \\
\hline WVE14880-12 & \begin{tabular}{l|l|}
29517 & WVE14880 \\
\end{tabular} & 8/5/08 13:01 & 281.7853 & 12 \\
\hline WVE14880-13 & 29734 $\mid$ WVE14880 & 8/7/08 20:33 & 281.8028 & 13 \\
\hline WVE14880-14 & \begin{tabular}{|l|l|}
29876 & WVE14880 \\
\end{tabular} & 8/14/08 16:02 & 281.8353 & 14 \\
\hline WVE14880-15 & \begin{tabular}{l|l|}
30046 & WVE14880
\end{tabular} & $8 / 21 / 08$ 10:11 & 281.8612 & 15 \\
\hline WVE14880-16 & 30301 WVE14880 & 8/28/08 9:48 & 281.8726 & 16 \\
\hline WVE14880-17 & \begin{tabular}{l|l}
30522 & WVE14880 \\
\end{tabular} & 9/3/08 14:59 & 281.9055 & 17 \\
\hline
\end{tabular}




\begin{tabular}{|c|c|c|c|c|}
\hline WVE14881-1 & $28315 \mid$ WVE14881 & 7/18/08 16:23 & 288.078 & 1 \\
\hline WVE14881-2 & \begin{tabular}{l|l|}
28447 & WVE14881 \\
\end{tabular} & $7 / 21 / 08$ 12:09 & 288.0816 & 2 \\
\hline WVE14881-3 & \begin{tabular}{|l|l|}
28529 & $W V 14881$ \\
\end{tabular} & $7 / 22 / 08$ 12:45 & 288.0817 & $\overline{3}$ \\
\hline WVE14881-4 & \begin{tabular}{l|l|}
28603 & WVE14881 \\
\end{tabular} & $7 / 23 / 08$ 11:55 & 288.0825 & 4 \\
\hline WVE14881-5 & 28940 $\mid$ WVE14881 & $7 / 26 / 08$ 15:34 & 288.0418 & 5 \\
\hline WVE14881-6 & 28991|WVE14881 & $7 / 27 / 08$ 14:29 & 288.0414 & 6 \\
\hline WVE14881-7 & $29045 \mid$ WVE14881 & $7 / 28 / 08$ 18:26 & 288.033 & 7 \\
\hline WVE14881-8 & \begin{tabular}{l|l|}
29113 & WVE14881
\end{tabular} & $7 / 29 / 08$ 12:28 & 288.0375 & 8 \\
\hline WVE14881-9 & \begin{tabular}{|l|l|}
29380 & WVE14881 \\
\end{tabular} & $8 / 2 / 0814: 28$ & 287.1848 & 9 \\
\hline WVE14881-10 & \begin{tabular}{l|l|}
29425 & WVE14881
\end{tabular} & $8 / 3 / 08$ 12:34 & 287.1942 & 10 \\
\hline WVE14881-11 & \begin{tabular}{l|l|l|}
29483 & WVE14881 \\
\end{tabular} & 8/4/08 13:18 & 287.205 & 11 \\
\hline WVE14881-12 & 29518|WVE14881 & 8/5/08 13:02 & 287.2091 & 12 \\
\hline WVE14881-13 & \begin{tabular}{l|l|}
29731 & WVE14881 \\
\end{tabular} & 8/7/08 20:27 & 287.2182 & 13 \\
\hline WVE14881-14 & \begin{tabular}{l|l|l|}
29877 & WVE14881
\end{tabular} & $8 / 14 / 08$ 16:03 & 287.2634 & 14 \\
\hline WVE14881-15 & \begin{tabular}{l|l|}
30047 & WVE14881 \\
\end{tabular} & 8/21/08 10:11 & 287.3085 & 15 \\
\hline WVE14881-16 & \begin{tabular}{l|l|}
30302 & WVE14881
\end{tabular} & 8/28/08 9:48 & 287.3451 & 16 \\
\hline WVE14881-17 & \begin{tabular}{l|l}
30523 & WVE14881 \\
\end{tabular} & 9/3/08 15:00 & 287.374 & 17 \\
\hline WVE14882-1 & \begin{tabular}{l|l|} 
28316 & WVE14882
\end{tabular} & $7 / 18 / 08$ 16:24 & 291.3378 & 1 \\
\hline WVE14882-2 & \begin{tabular}{l|l}
28448 & WVE14882 \\
\end{tabular} & $7 / 21 / 08$ 12:10 & 291.3456 & 2 \\
\hline WVE14882-3 & 28530|WVE14882 & $7 / 22 / 08$ 12:46 & 291.3407 & 3 \\
\hline WVE14882-4 & 28604 $\mid$ WVE14882 & $7 / 23 / 08$ 11:56 & 291.3416 & 4 \\
\hline WVE14882-5 & 28943 WVE14882 & $7 / 26 / 08$ 15:36 & 291.3037 & 5 \\
\hline WVE14882-6 & 28988 $\mid$ WVE14882 & 7/27/08 14:27 & 291.3025 & 6 \\
\hline WVE14882-7 & \begin{tabular}{l|l|l|}
29042 & WVE14882 \\
\end{tabular} & $7 / 28 / 08$ 18:24 & 291.2968 & 7 \\
\hline WVE14882-8 & 29110|WVE14882 & 7/29/08 12:26 & 291.2976 & 8 \\
\hline WVE14882-9 & \begin{tabular}{l|l|l|}
29381 & WVE14882 \\
\end{tabular} & $8 / 2 / 0814: 29$ & 290.4398 & 9 \\
\hline WVE14882-10 & \begin{tabular}{l|l|}
29426 & WVE14882
\end{tabular} & 8/3/08 12:35 & 290.4527 & 10 \\
\hline WVE14882-11 & 29484|WVE14882 & 8/4/08 13:18 & 290.4634 & 11 \\
\hline WVE14882-12 & \begin{tabular}{|l|l|}
29520 & WVE14882 \\
\end{tabular} & 8/5/08 13:03 & 290.4737 & 12 \\
\hline WVE14882-13 & \begin{tabular}{l|l|l|}
29735 & WVE14882 \\
\end{tabular} & 8/7/08 20:34 & 290.4797 & 13 \\
\hline WVE14882-14 & \begin{tabular}{l|l|}
29878 & WVE14882 \\
\end{tabular} & 8/14/08 16:04 & 290.5222 & 14 \\
\hline WVE14882-15 & \begin{tabular}{l|l|l|}
30048 & WVE14882
\end{tabular} & 8/21/08 10:12 & 290.5688 & 15 \\
\hline WVE14882-16 & \begin{tabular}{l|l}
30303 & WVE14882
\end{tabular} & 8/28/08 9:49 & 290.6106 & 16 \\
\hline WVE14882-17 & \begin{tabular}{l|l|l|}
30524 & WVE14882
\end{tabular} & 9/3/08 15:01 & 290.6351 & 17 \\
\hline WVE14883-1 & \begin{tabular}{l|l|l|}
28317 & WVE14883
\end{tabular} & 7/18/08 16:25 & 285.8819 & 1 \\
\hline WVE14883-2 & $28449 \mid$ WVE14883 & 7/21/08 12:10 & 285.8875 & 2 \\
\hline WVE14883-3 & \begin{tabular}{l|l|l|}
28531 & WVE14883 \\
\end{tabular} & $7 / 22 / 08$ 12:47 & 285.8863 & 3 \\
\hline WVE14883-4 & $28605 \mid$ WVE14883 & $7 / 23 / 08$ 11:57 & 285.8857 & 4 \\
\hline WVE14883-5 & 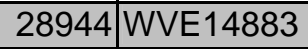 & 7/26/08 15:37 & 285.8414 & 5 \\
\hline WVE14883-6 & 28987|WVE14883 & $7 / 27 / 08$ 14:26 & 285.8369 & 6 \\
\hline WVE14883-7 & \begin{tabular}{l|l|l|}
29041 & WVE14883 \\
\end{tabular} & $7 / 28 / 0818: 23$ & 285.8387 & 7 \\
\hline WVE14883-8 & $29109 \mid$ WVE14883 & $7 / 29 / 08$ 12:25 & 285.8399 & 8 \\
\hline WVE14883-9 & \begin{tabular}{l|l|l}
29680 & WVE14883
\end{tabular} & 8/7/08 19:51 & 285.849 & 9 \\
\hline WVE14883-10 & \begin{tabular}{l|l|l|}
29862 & WVE14883
\end{tabular} & $8 / 14 / 08$ 15:52 & 285.8461 & 10 \\
\hline WVE14883-11 & 30032 WVE14883 & 8/21/08 10:00 & 285.8495 & 11 \\
\hline WVE14883-12 & \begin{tabular}{l|l|l|}
30287 & WVE14883
\end{tabular} & 8/28/08 9:37 & 285.8546 & 12 \\
\hline WVE14883-13 & \begin{tabular}{|l|l|}
30508 & WVE14883
\end{tabular} & 9/3/08 14:43 & 285.8575 & 13 \\
\hline WVE14991-1 & \begin{tabular}{l|l|} 
28320 & WVE14991
\end{tabular} & $7 / 18 / 08$ 16:29 & 280.104 & 1 \\
\hline WVE14991-2 & \begin{tabular}{l|l}
28452 & WVE14991
\end{tabular} & $7 / 21 / 08$ 12:14 & 280.0962 & 2 \\
\hline
\end{tabular}




\begin{tabular}{|c|c|c|c|c|}
\hline WVE14991-3 & \begin{tabular}{l|l|} 
28534 & WVE14991
\end{tabular} & $7 / 22 / 08$ 12:51 & 280.0971 & 3 \\
\hline WVE14991-4 & \begin{tabular}{l|l|}
28608 & WVE14991
\end{tabular} & 7/23/08 11:58 & 280.0998 & 4 \\
\hline WVE14991-5 & \begin{tabular}{|l|l|}
28650 & WVE14991
\end{tabular} & $7 / 24 / 08$ 12:21 & 280.0998 & 5 \\
\hline WVE14991-6 & \begin{tabular}{l|l|}
28694 & WVE14991
\end{tabular} & 7/24/08 18:12 & 280.0999 & 6 \\
\hline WVE14991-7 & 28720|WVE14991 & $7 / 25 / 08$ 13:07 & 280.0978 & 7 \\
\hline WVE14991-8 & 28908 $\mid$ WVE14991 & 7/26/08 14:58 & 280.1004 & 8 \\
\hline WVE14991-9 & 29704 $\mid$ WVE14991 & 8/7/08 20:08 & 280.1143 & 9 \\
\hline WVE14991-10 & \begin{tabular}{|l|l|}
29903 & WVE14991
\end{tabular} & $8 / 14 / 08$ 16:25 & 280.1175 & 10 \\
\hline WVE14991-11 & \begin{tabular}{|l|l|}
30069 & WVE14991 \\
\end{tabular} & $8 / 21 / 08$ 10:30 & 280.1158 & 11 \\
\hline WVE14991-12 & 30324 $\mid$ WVE14991 & 8/28/08 10:06 & 280.1181 & 12 \\
\hline WVE14991-13 & 30549 WVE14991 & 9/3/08 15:25 & 280.1197 & 13 \\
\hline WVE14992-1 & 28321 $\mid$ WVE14992 & $7 / 18 / 08$ 16:30 & 278.7323 & 1 \\
\hline WVE14992-2 & 28453|WVE14992 & $7 / 21 / 08$ 12:14 & 278.73 & 2 \\
\hline WVE14992-3 & \begin{tabular}{l|l|l|}
28535 & WVE14992
\end{tabular} & $7 / 22 / 08$ 12:52 & 278.731 & 3 \\
\hline WVE14992-4 & $28609 \mid$ WVE14992 & $7 / 23 / 08$ 11:59 & 278.7356 & 4 \\
\hline WVE14992-5 & \begin{tabular}{l|l|l|}
28655 & WVE14992
\end{tabular} & $7 / 24 / 08$ 12:25 & 278.7316 & 5 \\
\hline WVE14992-6 & 28699|WVE14992 & $7 / 24 / 08$ 18:16 & 278.732 & 6 \\
\hline WVE14992-7 & 28729|WVE14992 & $7 / 25 / 08$ 13:14 & 278.728 & 7 \\
\hline WVE14992-8 & 28925|WVE14992 & $7 / 26 / 08$ 15:10 & 278.7368 & 8 \\
\hline WVE14992-9 & \begin{tabular}{|l|l|}
29697 & WVE14992 \\
\end{tabular} & 8/7/08 20:03 & 278.7488 & 9 \\
\hline WVE14992-10 & \begin{tabular}{l|l|l}
29867 & WVE14992
\end{tabular} & $8 / 14 / 08$ 15:56 & 278.7436 & 10 \\
\hline WVE14992-11 & \begin{tabular}{l|l|l|}
30037 & WVE14992 \\
\end{tabular} & 8/21/08 10:04 & 278.7482 & 11 \\
\hline WVE14992-12 & \begin{tabular}{l|l}
30292 & WVE14992
\end{tabular} & 8/28/08 9:40 & 278.7578 & 12 \\
\hline WVE14992-13 & \begin{tabular}{l|l}
30513 & WVE14992
\end{tabular} & 9/3/08 14:48 & 278.7663 & 13 \\
\hline WVE14993-1 & 28322 $\mid$ WVE14993 & $7 / 18 / 08$ 16:31 & 280.8313 & 1 \\
\hline WVE14993-2 & 28454 WVE14993 & $7 / 21 / 08$ 12:15 & 280.8271 & 2 \\
\hline WVE14993-3 & 28536|WVE14993 & $7 / 22 / 08$ 12:53 & 280.8279 & 3 \\
\hline WVE14993-4 & 28610|WVE14993 & 7/23/08 12:00 & 280.8283 & 4 \\
\hline WVE14993-5 & 28953|WVE14993 & $7 / 26 / 08$ 15:43 & 280.7928 & 5 \\
\hline WVE14993-6 & \begin{tabular}{|l|l|}
28982 & WVE14993
\end{tabular} & $7 / 27 / 08$ 14:22 & 280.7906 & 6 \\
\hline WVE14993-7 & \begin{tabular}{|l|l|}
29036 & WVE14993 \\
\end{tabular} & 7/28/08 18:20 & 280.7887 & 7 \\
\hline WVE14993-8 & 29104|WVE14993 & $7 / 29 / 08$ 12:22 & 280.7911 & 8 \\
\hline WVE14993-9 & 29382 $\mid$ WVE14993 & $8 / 2 / 08$ 14:29 & 280.0287 & 9 \\
\hline WVE14993-10 & \begin{tabular}{|l|l|}
29427 & WVE14993 \\
\end{tabular} & $8 / 3 / 0812: 36$ & 280.0394 & 10 \\
\hline WVE14993-11 & \begin{tabular}{|l|l|}
29485 & WVE14993 \\
\end{tabular} & 8/4/08 13:19 & 280.055 & 11 \\
\hline WVE14993-12 & $29519 \mid$ WVE14993 & 8/5/08 13:02 & 280.0625 & 12 \\
\hline WVE14993-13 & 29738|WVE14993 & 8/7/08 20:36 & 280.0748 & 13 \\
\hline WVE14993-14 & 29879 WVE14993 & $8 / 14 / 08$ 16:05 & 280.1014 & 14 \\
\hline WVE14993-15 & \begin{tabular}{|l|l|}
30049 & WVE14993 \\
\end{tabular} & 8/21/08 10:13 & 280.1563 & 15 \\
\hline WVE14993-16 & 30304 WVE14993 & 8/28/08 9:51 & 280.1972 & 16 \\
\hline WVE14993-17 & 30525|WVE14993 & 9/3/08 15:02 & 280.2239 & 17 \\
\hline WVE14994-1 & \begin{tabular}{l|l|} 
28323 & WVE14994
\end{tabular} & 7/18/08 16:32 & 280.2367 & 1 \\
\hline WVE14994-2 & 28455|WVE14994 & $7 / 21 / 08$ 12:16 & 280.2215 & 2 \\
\hline WVE14994-3 & \begin{tabular}{l|l|}
28537 & WVE14994 \\
\end{tabular} & $7 / 22 / 08$ 12:54 & 280.2238 & 3 \\
\hline WVE14994-4 & \begin{tabular}{l|l}
28611 & WVE14994
\end{tabular} & 7/23/08 12:00 & 280.2276 & 4 \\
\hline WVE14994-5 & \begin{tabular}{|l|l|}
28660 & WVE14994 \\
\end{tabular} & 7/24/08 12:30 & 280.1876 & 5 \\
\hline WVE14994-6 & \begin{tabular}{l|l|l|}
28705 & WVE14994
\end{tabular} & 7/24/08 18:20 & 280.1867 & 6 \\
\hline WVE14994-7 & \begin{tabular}{l|l|l|}
28734 & WVE14994 \\
\end{tabular} & $7 / 25 / 08$ 13:18 & 280.1799 & 7 \\
\hline WVE14994-8 & \begin{tabular}{l|l|}
28913 & WVE14994 \\
\end{tabular} & 7/26/08 15:02 & 280.1822 & 8 \\
\hline
\end{tabular}




\begin{tabular}{|c|c|c|c|c|}
\hline WVE14994-9 & \begin{tabular}{|l|l|}
29336 & WVE14994 \\
\end{tabular} & 8/1/08 14:02 & 279.4304 & 9 \\
\hline WVE14994-10 & \begin{tabular}{l|l|l|}
29405 & WVE14994 \\
\end{tabular} & $8 / 2 / 08$ 14:51 & 279.4432 & 10 \\
\hline WVE14994-11 & \begin{tabular}{|l|l|}
$2945 V E 14994$ \\
\end{tabular} & $8 / 3 / 08$ 12:53 & 279.4497 & 11 \\
\hline WVE14994-12 & \begin{tabular}{l|l|l|}
29475 & WVE14994 \\
\end{tabular} & 8/4/08 13:06 & 279.4588 & 12 \\
\hline WVE14994-13 & \begin{tabular}{l|l|}
29728 & WVE14994 \\
\end{tabular} & 8/7/08 20:25 & 279.4737 & 13 \\
\hline WVE14994-14 & 29889 WVE14994 & $8 / 14 / 08$ 16:12 & 279.5073 & 14 \\
\hline WVE14994-15 & \begin{tabular}{l|l|l}
30059 & WVE14994 \\
\end{tabular} & 8/21/08 10:21 & 279.536 & 15 \\
\hline WVE14994-16 & \begin{tabular}{l|l|l|}
30314 & WVE14994
\end{tabular} & 8/28/08 9:57 & 279.5757 & 16 \\
\hline WVE14994-17 & \begin{tabular}{l|l|l}
30535 & WVE14994 \\
\end{tabular} & 9/3/08 15:12 & 279.6023 & 17 \\
\hline WVE14995-1 & 28324 $\mid$ WVE14995 & 7/18/08 16:32 & 281.456 & 1 \\
\hline WVE14995-2 & \begin{tabular}{|l|l|}
28456 & WVE14995 \\
\end{tabular} & $7 / 21 / 08$ 12:17 & 281.4413 & 2 \\
\hline WVE14995-3 & \begin{tabular}{l|l}
28538 & WVE14995
\end{tabular} & $7 / 22 / 08$ 12:55 & 281.4468 & 3 \\
\hline WVE14995-4 & \begin{tabular}{l|l|l|}
28612 & WVE14995 \\
\end{tabular} & 7/23/08 12:01 & 281.4476 & 4 \\
\hline WVE14995-5 & \begin{tabular}{l|l|l|}
28661 & WVE14995 \\
\end{tabular} & 7/24/08 12:31 & 281.4531 & 5 \\
\hline WVE14995-6 & \begin{tabular}{l|l|l|}
28706 & WVE14995 \\
\end{tabular} & $7 / 24 / 08$ 18:21 & 281.4499 & 6 \\
\hline WVE14995-7 & \begin{tabular}{l|l|l|}
28735 & WVE14995 \\
\end{tabular} & 7/25/08 13:19 & 281.4493 & 7 \\
\hline WVE14995-8 & \begin{tabular}{l|l|l|}
28914 & WVE14995
\end{tabular} & 7/26/08 15:02 & 281.4477 & 8 \\
\hline WVE14995-9 & 29339|WVE14995 & $8 / 1 / 08$ 14:05 & 280.7381 & 9 \\
\hline WVE14995-10 & \begin{tabular}{l|l|}
29408 & WVE14995 \\
\end{tabular} & $8 / 2 / 0814: 53$ & 280.7474 & 10 \\
\hline WVE14995-11 & \begin{tabular}{l|l|l|}
29451 & WVE14995
\end{tabular} & $8 / 3 / 0812: 53$ & 280.757 & 11 \\
\hline WVE14995-12 & \begin{tabular}{l|l|l|}
29476 & WVE14995 \\
\end{tabular} & 8/4/08 13:07 & 280.7697 & 12 \\
\hline WVE14995-13 & \begin{tabular}{l|l|l|}
29729 & WVE14995 \\
\end{tabular} & $8 / 7 / 0820: 26$ & 280.7835 & 13 \\
\hline WVE14995-14 & \begin{tabular}{l|l|l}
29890 & WVE14995
\end{tabular} & 8/14/08 16:13 & 280.8144 & 14 \\
\hline WVE14995-15 & \begin{tabular}{|l|l|l|}
30060 & WVE14995 \\
\end{tabular} & 8/21/08 10:22 & 280.8391 & 15 \\
\hline WVE14995-16 & \begin{tabular}{l|l|l|}
30315 & WVE14995 \\
\end{tabular} & 8/28/08 9:58 & 280.8735 & 16 \\
\hline WVE14995-17 & \begin{tabular}{l|l|l|}
30536 & WVE14995 \\
\end{tabular} & 9/3/08 15:14 & 280.9 & 17 \\
\hline WVE14996-1 & \begin{tabular}{l|l|l|}
28325 & WVE14996 \\
\end{tabular} & 7/18/08 16:33 & 281.3381 & 1 \\
\hline WVE14996-2 & \begin{tabular}{l|l|l|}
28457 & WVE14996
\end{tabular} & $7 / 21 / 08$ 12:18 & 281.3317 & 2 \\
\hline WVE14996-3 & 28539 WVE14996 & $7 / 22 / 08$ 12:56 & 281.3324 & 3 \\
\hline WVE14996-4 & \begin{tabular}{l|l|l|}
28613 & WVE14996 \\
\end{tabular} & $7 / 23 / 08$ 12:02 & 281.3319 & 4 \\
\hline WVE14996-5 & \begin{tabular}{l|l}
28662 & WVE14996
\end{tabular} & $7 / 24 / 08$ 12:32 & 281.3319 & 5 \\
\hline WVE14996-6 & \begin{tabular}{l|l|l|}
28707 & WVE14996
\end{tabular} & $7 / 24 / 08$ 18:22 & 281.3302 & 6 \\
\hline WVE14996-7 & \begin{tabular}{|l|l|}
28736 & WVE14996 \\
\end{tabular} & 7/25/08 13:20 & 281.3291 & 7 \\
\hline WVE14996-8 & \begin{tabular}{l|l|l|} 
28922 & WVE14996
\end{tabular} & 7/26/08 15:08 & 281.3306 & 8 \\
\hline WVE14996-9 & \begin{tabular}{l|l|l|}
29340 & WVE14996 \\
\end{tabular} & $8 / 1 / 08$ 14:06 & 280.6133 & 9 \\
\hline WVE14996-10 & \begin{tabular}{l|l|l|}
29409 & WVE14996 \\
\end{tabular} & $8 / 2 / 0814: 54$ & 280.6217 & 10 \\
\hline WVE14996-11 & \begin{tabular}{l|l|l|}
29455 & WVE14996 \\
\end{tabular} & $8 / 3 / 08$ 12:56 & 280.6275 & 11 \\
\hline WVE14996-12 & \begin{tabular}{l|l|l|}
29477 & WVE14996
\end{tabular} & 8/4/08 13:08 & 280.6337 & 12 \\
\hline WVE14996-13 & 29730 WVE14996 & 8/7/08 20:26 & 280.6448 & 13 \\
\hline WVE14996-14 & 29891 $\mid$ WVE14996 & $8 / 14 / 08$ 16:14 & 280.6829 & 14 \\
\hline WVE14996-15 & \begin{tabular}{l|l|}
30061 & WVE14996 \\
\end{tabular} & $8 / 21 / 08$ 10:23 & 280.7177 & 15 \\
\hline WVE14996-16 & \begin{tabular}{l|l|l}
30316 & WVE14996 \\
\end{tabular} & 8/28/08 9:59 & 280.7534 & 16 \\
\hline WVE14996-17 & \begin{tabular}{l|l|l|}
30537 & WVE14996 \\
\end{tabular} & 9/3/08 15:15 & 280.7749 & 17 \\
\hline WVE14997-1 & \begin{tabular}{l|l|l}
28326 & WVE14997 \\
\end{tabular} & 7/18/08 16:34 & 281.8902 & 1 \\
\hline WVE14997-2 & \begin{tabular}{l|l|}
28458 & WVE14997 \\
\end{tabular} & $7 / 21 / 08$ 12:19 & 281.8856 & 2 \\
\hline WVE14997-3 & \begin{tabular}{|l|l|}
28540 & WVE14997 \\
\end{tabular} & $7 / 22 / 08$ 12:57 & 281.8891 & 3 \\
\hline WVE14997-4 & 28614 $\mid$ WVE14997 & $7 / 23 / 08$ 12:02 & 281.8911 & 4 \\
\hline WVE14997-5 & 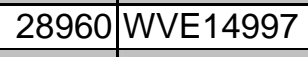 & 7/26/08 15:48 & 281.8836 & 5 \\
\hline WVE14997-6 & \begin{tabular}{l|l|l} 
28980 & WVE14997
\end{tabular} & $7 / 27 / 08$ 14:21 & 281.8808 & 6 \\
\hline
\end{tabular}




\begin{tabular}{|c|c|c|c|c|}
\hline WVE14997-7 & \begin{tabular}{l|l|l} 
29034 & WVE14997
\end{tabular} & $7 / 28 / 08$ 18:19 & 281.8788 & 7 \\
\hline WVE14997-8 & \begin{tabular}{l|l|}
29102 & WVE14997 \\
\end{tabular} & $7 / 29 / 08$ 12:21 & 281.8796 & 8 \\
\hline WVE14997-9 & \begin{tabular}{|l|l|}
29329 & WVE14997
\end{tabular} & 8/1/08 13:57 & 281.1353 & 9 \\
\hline WVE14997-10 & \begin{tabular}{|l|l|} 
29398 & WVE14997
\end{tabular} & $8 / 2 / 08$ 14:45 & 281.1456 & 10 \\
\hline WVE14997-11 & 29444|WVE14997 & $8 / 3 / 08$ 12:48 & 281.1531 & 11 \\
\hline WVE14997-12 & 29468 $\mid$ WVE14997 & 8/4/08 13:01 & 281.1565 & 12 \\
\hline WVE14997-13 & 29719|WVE14997 & 8/7/08 20:18 & 281.173 & 13 \\
\hline WVE14997-14 & 29871|WVE14997 & 8/14/08 15:59 & 281.2144 & 14 \\
\hline WVE14997-15 & \begin{tabular}{|l|l|}
30043 & WVE14997 \\
\end{tabular} & $8 / 21 / 08$ 10:09 & 281.2433 & 15 \\
\hline WVE14997-16 & \begin{tabular}{|l|l|}
30296 & WVE14997 \\
\end{tabular} & |8/28/08 9:43 & 281.2774 & 16 \\
\hline WVE14997-17 & 30517 WVE14997 & 9/3/08 14:54 & 281.2954 & 17 \\
\hline WVE14998-1 & \begin{tabular}{|l|l|}
28327 & WVE14998 \\
\end{tabular} & $7 / 18 / 08$ 16:35 & 282.549 & 1 \\
\hline WVE14998-2 & 28459|WVE14998 & $7 / 21 / 08$ 12:19 & 282.5408 & 2 \\
\hline WVE14998-3 & \begin{tabular}{l|l|}
28541 & WVE14998
\end{tabular} & $7 / 22 / 08$ 12:58 & 282.5464 & 3 \\
\hline WVE14998-4 & 28615|WVE14998 & $7 / 23 / 08$ 12:03 & 282.543 & 4 \\
\hline WVE14998-5 & \begin{tabular}{l|l|l|}
28959 & WVE14998
\end{tabular} & 7/26/08 15:47| & 282.5482 & 5 \\
\hline WVE14998-6 & 28979|WVE14998 & $7 / 27 / 08$ 14:20 & 282.5442 & 6 \\
\hline WVE14998-7 & 29033 $\mid$ WVE14998 & $7 / 28 / 08$ 18:18 & 282.541 & 7 \\
\hline WVE14998-8 & 29101|WVE14998 & $7 / 29 / 08$ 12:20 & 282.5425 & 8 \\
\hline WVE14998-9 & \begin{tabular}{|l|l|}
29330 & WVE14998 \\
\end{tabular} & 8/1/08 13:57 & 281.824 & 9 \\
\hline WVE14998-10 & 29399|WVE14998 & $8 / 2 / 08$ 14:46 & 281.8366 & 10 \\
\hline WVE14998-11 & \begin{tabular}{|l|l|l|}
29445 & WVE14998 \\
\end{tabular} & $8 / 3 / 08$ 12:48 & 281.8485 & 11 \\
\hline WVE14998-12 & 29469|WVE14998 & 8/4/08 13:02 & 281.8545 & 12 \\
\hline WVE14998-13 & 29720|WVE14998 & 8/7/08 20:19 & 281.8658 & 13 \\
\hline WVE14998-14 & 29872|WVE14998 & 8/14/08 16:00 & 281.8931 & 14 \\
\hline WVE14998-15 & \begin{tabular}{|l|l|}
30042 & WVE14998 \\
\end{tabular} & 8/21/08 10:08 & 281.9246 & 15 \\
\hline WVE14998-16 & 30297 $\mid$ WVE14998 & 8/28/08 9:44 & 281.9574 & 16 \\
\hline WVE14998-17 & 30518 WVE14998 & 9/3/08 14:55 & 281.9827 & 17 \\
\hline WVE14999-1 & 28328|WVE14999 & $7 / 18 / 08$ 16:36 & 278.4425 & 1 \\
\hline WVE14999-2 & 28460 $\mid$ WVE14999 & $7 / 21 / 08$ 12:20 & 278.4342 & 2 \\
\hline WVE14999-3 & 28542 $\mid$ WVE14999 & $7 / 22 / 08$ 12:59 & 278.4382 & 3 \\
\hline WVE14999-4 & \begin{tabular}{l|l|l} 
28616 & WVE14999
\end{tabular} & $7 / 23 / 08$ 12:04 & 278.4364 & 4 \\
\hline WVE14999-5 & 28958|WVE14999 & $7 / 26 / 08$ 15:47| & 278.4469 & 5 \\
\hline WVE14999-6 & \begin{tabular}{l|l|}
28978 & WVE14999
\end{tabular} & $7 / 27 / 08$ 14:20 & 278.4465 & 6 \\
\hline WVE14999-7 & \begin{tabular}{|l|l|}
29032 & WVE14999 \\
\end{tabular} & 7/28/08 18:17 & 278.4397 & 7 \\
\hline WVE14999-8 & \begin{tabular}{|l|l|}
29100 & WVE14999 \\
\end{tabular} & 7/29/08 12:20 & 278.4407 & 8 \\
\hline WVE14999-9 & 29331 $\mid$ WVE14999 & 8/1/08 13:58 & 277.7416 & 9 \\
\hline WVE14999-10 & 29400|WVE14999 & $8 / 2 / 08$ 14:47 & 277.7597 & 10 \\
\hline WVE14999-11 & \begin{tabular}{|l|l|}
29446 & WVE14999 \\
\end{tabular} & 8/3/08 12:49 & 277.7614 & 11 \\
\hline WVE14999-12 & 29470 $\mid$ WVE14999 & 8/4/08 13:02 & 277.7744 & 12 \\
\hline WVE14999-13 & 29721 $\mid$ WVE14999 & 8/7/08 20:20 & 277.7871 & 13 \\
\hline WVE14999-14 & \begin{tabular}{l|l|}
29873 & WVE14999
\end{tabular} & 8/14/08 16:00 & 277.8185 & 14 \\
\hline WVE14999-15 & $30041 \mid$ WVE14999 & $8 / 21 / 08$ 10:07 & 277.8504 & 15 \\
\hline WVE14999-16 & 30298|WVE14999 & 8/28/08 9:45 & 277.8861 & 16 \\
\hline WVE14999-17 & $30519 \mid$ WVE14999 & 9/3/08 14:56 & 277.9237 & 17 \\
\hline WVE15000-1 & \begin{tabular}{|l|l|}
28329 & WVE15000 \\
\end{tabular} & 7/18/08 16:37 & 280.5909 & 1 \\
\hline WVE15000-2 & $28461 \mid$ WVE15000 & $7 / 21 / 08$ 12:21 & 280.5823 & 2 \\
\hline WVE15000-3 & \begin{tabular}{|l|l|}
28543 & WVE15000 \\
\end{tabular} & $7 / 22 / 08$ 13:00 & 280.5831 & 3 \\
\hline WVE15000-4 & \begin{tabular}{l|l|}
28617 & WVE15000 \\
\end{tabular} & 7/23/08 12:04 & 280.5864 & 4 \\
\hline
\end{tabular}




\begin{tabular}{|c|c|c|c|c|}
\hline WVE15000-5 & 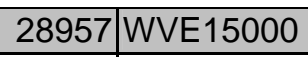 & $7 / 26 / 08$ 15:46 & 280.5871 & 5 \\
\hline WVE15000-6 & \begin{tabular}{l|l|l|}
28977 & WVE15000
\end{tabular} & $7 / 27 / 0814: 19$ & 280.5825 & 6 \\
\hline WVE15000-7 & \begin{tabular}{|l|l|}
29031 & WVE15000 \\
\end{tabular} & 7/28/08 18:17 & 280.5778 & 7 \\
\hline WVE15000-8 & \begin{tabular}{l|l|}
29099 & WVE15000 \\
\end{tabular} & $7 / 29 / 08$ 12:19 & 280.5835 & 8 \\
\hline WVE15000-9 & \begin{tabular}{l|l|} 
29332 & WVE15000
\end{tabular} & 8/1/08 13:59 & 279.8364 & 9 \\
\hline WVE15000-10 & 29401|WVE15000 & $8 / 2 / 08$ 14:47 & 279.8442 & 10 \\
\hline WVE15000-11 & \begin{tabular}{l|l|l|}
29447 & WV15000
\end{tabular} & $8 / 3 / 08$ 12:50 & 279.85 & 11 \\
\hline WVE15000-12 & \begin{tabular}{l|l|}
29471 & WVE15000
\end{tabular} & 8/4/08 13:03 & 279.8595 & 12 \\
\hline WVE15000-13 & \begin{tabular}{|l|l|}
29722 & WVE15000 \\
\end{tabular} & $8 / 7 / 0820: 21$ & 279.8739 & 13 \\
\hline WVE15000-14 & 29874|WVE15000 & 8/14/08 16:01 & 279.9101 & 14 \\
\hline WVE15000-15 & \begin{tabular}{l|l|l}
30040 & WVE15000 \\
\end{tabular} & $8 / 21 / 08$ 10:06 & 279.9439 & 15 \\
\hline WVE15000-16 & 30299|WVE15000 & $8 / 28 / 089: 46$ & 279.9669 & 16 \\
\hline WVE15000-17 & \begin{tabular}{l|l|l}
30520 & WVE15000
\end{tabular} & 9/3/08 14:57 & 279.9992 & 17 \\
\hline WVE15601-1 & \begin{tabular}{l|l|l}
27727 & WVE15601
\end{tabular} & $7 / 11 / 08$ 18:26 & 275.2965 & 1 \\
\hline WVE15601-2 & \begin{tabular}{l|l|l|}
27774 & WVE15601 \\
\end{tabular} & 7/12/08 13:04 & 275.2871 & 2 \\
\hline WVE15601-3 & \begin{tabular}{l|l|}
27802 & WVE15601
\end{tabular} & $7 / 13 / 08$ 15:00 & 275.2816 & 3 \\
\hline WVE15601-4 & \begin{tabular}{l|l|}
27933 & WVE15601 \\
\end{tabular} & $7 / 14 / 08$ 14:21 & 275.2838 & 4 \\
\hline WVE15601-5 & \begin{tabular}{l|l|} 
27992 & WVE15601
\end{tabular} & $7 / 15 / 08$ 13:30 & 275.3191 & 5 \\
\hline WVE15601-6 & \begin{tabular}{l|l}
28018 & WVE15601 \\
\end{tabular} & 7/15/08 19:17 & 275.2975 & 6 \\
\hline WVE15601-7 & \begin{tabular}{l|l|}
28081 & WVE15601
\end{tabular} & 7/16/08 19:20 & 275.2895 & 7 \\
\hline WVE15601-8 & 28115 $\mid$ WVE15601 & $7 / 17 / 08$ 14:40 & 275.2885 & 8 \\
\hline WVE15601-9 & \begin{tabular}{l|l|}
28260 & WVE15601 \\
\end{tabular} & $7 / 18 / 08$ 15:29 & 275.2903 & 9 \\
\hline WVE15601-10 & \begin{tabular}{l|l} 
28392 & WVE15601
\end{tabular} & 7/21/08 11:19 & 275.2871 & 10 \\
\hline WVE15601-11 & \begin{tabular}{l|l|l|}
28740 & WVE15601 \\
\end{tabular} & $7 / 25 / 08$ 13:22 & 275.2848 & 11 \\
\hline WVE15601-12 & 29255 WVE15601 & 7/30/08 12:30 & 275.287 & 12 \\
\hline WVE15601-13 & \begin{tabular}{l|l|l|}
29654 & WVE15601 \\
\end{tabular} & 8/7/08 19:32 & 275.2864 & 13 \\
\hline WVE15601-14 & 29931|WVE15601 & 8/14/08 16:43 & 275.2896 & 14 \\
\hline WVE15601-15 & \begin{tabular}{l|l|l}
30102 & WVE15601
\end{tabular} & $8 / 21 / 08$ 10:55 & 275.2882 & 15 \\
\hline WVE15601-16 & \begin{tabular}{l|l}
30356 & WVE15601
\end{tabular} & 8/28/08 10:33 & 275.2857 & 16 \\
\hline WVE15601-17 & \begin{tabular}{l|l|}
30580 & WVE15601 \\
\end{tabular} & 9/3/08 17:00 & 275.2915 & 17 \\
\hline WVE15602-1 & \begin{tabular}{l|l}
27728 & WVE15602 \\
\end{tabular} & 7/11/08 18:27 & 278.7718 & 1 \\
\hline WVE15602-2 & $27775 \mid$ WVE15602 & $7 / 12 / 08$ 13:05 & 278.763 & 2 \\
\hline WVE15602-3 & \begin{tabular}{l|l|l}
27803 & WVE15602
\end{tabular} & $7 / 13 / 08$ 15:00 & 278.7615 & 3 \\
\hline WVE15602-4 & \begin{tabular}{l|l|}
27934 & WVE15602 \\
\end{tabular} & $7 / 14 / 08$ 14:22 & 278.7626 & 4 \\
\hline WVE15602-5 & 27993 $\mid$ WVE15602 & 7/15/08 13:31 & 278.7869 & 5 \\
\hline WVE15602-6 & 28019 WVE15602 & 7/15/08 19:18 & 278.7774 & 6 \\
\hline WVE15602-7 & \begin{tabular}{l|l|}
28082 & WVE15602 \\
\end{tabular} & 7/16/08 19:21 & 278.768 & 7 \\
\hline WVE15602-8 & \begin{tabular}{l|l|l} 
28116 & WVE15602
\end{tabular} & $7 / 17 / 08$ 14:41 & 278.7697 & 8 \\
\hline WVE15602-9 & \begin{tabular}{l|l|}
28261 & WVE15602 \\
\end{tabular} & $7 / 18 / 08$ 15:29 & 278.7712 & 9 \\
\hline WVE15602-10 & 28393|WVE15602 & $7 / 21 / 08$ 11:20 & 278.768 & 10 \\
\hline WVE15602-11 & \begin{tabular}{l|l|}
28741 & WVE15602 \\
\end{tabular} & $7 / 25 / 08$ 13:23 & 278.7642 & 11 \\
\hline WVE15602-12 & \begin{tabular}{l|l}
29256 & WVE15602
\end{tabular} & 7/30/08 12:31 & 278.7705 & 12 \\
\hline WVE15602-13 & 29655 WVE15602 & 8/7/08 19:33 & 278.7704 & 13 \\
\hline WVE15602-14 & 29932 WVE15602 & 8/14/08 16:44 & 278.7749 & 14 \\
\hline WVE15602-15 & \begin{tabular}{l|l|}
30103 & WVE15602 \\
\end{tabular} & $8 / 21 / 08$ 10:56 & 278.7758 & 15 \\
\hline WVE15602-16 & \begin{tabular}{l|l|l|}
30357 & WVE15602
\end{tabular} & $8 / 28 / 08$ 10:34 & 278.7811 & 16 \\
\hline WVE15602-17 & \begin{tabular}{l|l|l}
30579 & WVE15602
\end{tabular} & 9/3/08 17:00 & 278.7861 & 17 \\
\hline WVE15603-1 & 27729 WVE15603 & $7 / 11 / 08$ 18:28 & 278.1081 & 1 \\
\hline WVE15603-2 & \begin{tabular}{l|l|}
27776 & WVE15603 \\
\end{tabular} & 7/12/08 13:05 & 278.1016 & 2 \\
\hline
\end{tabular}




\begin{tabular}{|c|c|c|c|c|}
\hline WVE15603-3 & 27804 WVE15603 & 7/13/08 15:01 & 278.1014 & 3 \\
\hline WVE15603-4 & 27935|WVE15603 & $7 / 14 / 08$ 14:22 & 278.1011 & 4 \\
\hline WVE15603-5 & 27994|WVE15603 & 7/15/08 13:31 & 278.1249 & 5 \\
\hline WVE15603-6 & \begin{tabular}{l|l|l|}
28020 & WVE15603 \\
\end{tabular} & 7/15/08 19:18 & 278.1198 & 6 \\
\hline WVE15603-7 & 28083 WVE15603 & 7/16/08 19:22 & 278.1115 & 7 \\
\hline WVE15603-8 & 28117 $\mid$ WVE15603 & 7/17/08 14:42 & 278.1118 & 8 \\
\hline WVE15603-9 & 28262 WVE15603 & 7/18/08 15:30 & 278.113 & 9 \\
\hline WVE15603-10 & \begin{tabular}{l|l|l|}
28394 & WVE15603 \\
\end{tabular} & $7 / 21 / 08$ 11:20 & 278.1112 & 10 \\
\hline WVE15603-11 & 28742 $\mid$ WVE15603 & $7 / 25 / 08$ 13:24 & 278.1062 & 11 \\
\hline WVE15603-12 & 29257 WVE15603 & $7 / 30 / 08$ 12:32 & 278.1148 & 12 \\
\hline WVE15603-13 & \begin{tabular}{|l|l|}
29656 & WVE15603 \\
\end{tabular} & 8/7/08 19:33 & 278.1128 & 13 \\
\hline WVE15603-14 & 29933 WVE15603 & 8/14/08 16:44 & 278.1147 & 14 \\
\hline WVE15603-15 & 30104 WVE15603 & 8/21/08 10:57 & 278.1188 & 15 \\
\hline WVE15603-16 & 30358 WVE15603 & $8 / 28 / 08$ 10:35 & 278.1258 & 16 \\
\hline WVE15603-17 & 30578 $\mid$ WVE15603 & 9/3/08 16:59 & 278.1309 & 17 \\
\hline WVE15604-1 & 27730 WVE15604 & $7 / 11 / 08$ 18:29 & 279.4398 & 1 \\
\hline WVE15604-2 & 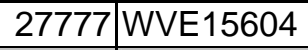 & 7/12/08 13:06 & 279.4304 & 2 \\
\hline WVE15604-3 & \begin{tabular}{l|l|l|}
27805 & WVE15604 \\
\end{tabular} & 7/13/08 15:02 & 279.4291 & 3 \\
\hline WVE15604-4 & \begin{tabular}{|l|l|}
27936 & WVE15604 \\
\end{tabular} & 7/14/08 14:23 & 279.4317 & 4 \\
\hline WVE15604-5 & $\begin{array}{l}27995 \\
\text { WVE15604 }\end{array}$ & 7/15/08 13:32 & 279.4555 & 5 \\
\hline WVE15604-6 & \begin{tabular}{l|l|l|}
28021 & WVE15604 \\
\end{tabular} & 7/15/08 19:19 & 279.4538 & 6 \\
\hline WVE15604-7 & 28084 WVE15604 & 7/16/08 19:23 & 279.4403 & 7 \\
\hline WVE15604-8 & 28118 $\mid$ WVE15604 & $7 / 17 / 08$ 14:42 & 279.4394 & 8 \\
\hline WVE15604-9 & 28263 WVE15604 & 7/18/08 15:31 & 279.4416 & 9 \\
\hline WVE15604-10 & 28395|WVE15604 & $7 / 21 / 08$ 11:21 & 279.4368 & 10 \\
\hline WVE15604-11 & \begin{tabular}{l|l|l|}
28743 & WVE15604 \\
\end{tabular} & $7 / 25 / 08$ 13:25 & 279.4341 & 11 \\
\hline WVE15604-12 & \begin{tabular}{l|l|l|}
29258 & WVE15604 \\
\end{tabular} & 7/30/08 12:33 & 279.4398 & 12 \\
\hline WVE15604-13 & 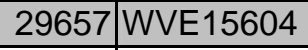 & 8/7/08 19:34 & 279.4426 & 13 \\
\hline WVE15604-14 & 29934 $\mid$ WVE15604 & $8 / 14 / 08$ 16:45 & 279.4445 & 14 \\
\hline WVE15604-15 & 30105 WVE15604 & 8/21/08 10:57 & 279.4487 & 15 \\
\hline WVE15604-16 & \begin{tabular}{l|l|l|}
30359 & WVE15604 \\
\end{tabular} & 8/28/08 10:36 & 279.4584 & 16 \\
\hline WVE15604-17 & 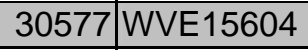 & 9/3/08 16:58 & 279.4632 & 17 \\
\hline WVE15652-1 & 27744 WVE15652 & 7/11/08 18:44 & 292.0444 & 1 \\
\hline WVE15652-2 & \begin{tabular}{l|l|l}
27781 & WVE15652 \\
\end{tabular} & 7/12/08 13:09 & 292.0355 & 2 \\
\hline WVE15652-3 & 27814|WVE15652 & 7/13/08 15:08 & 292.0336 & 3 \\
\hline WVE15652-4 & 27945 WVE15652 & 7/14/08 14:32 & 292.0332 & 4 \\
\hline WVE15652-5 & 27999 WVE15652 & 7/15/08 13:34 & 291.2234 & 5 \\
\hline WVE15652-6 & 28030 WVE15652 & 7/15/08 19:27 & 291.2305 & 6 \\
\hline WVE15652-7 & 28098 $\mid$ WVE15652 & 7/16/08 19:39 & 291.2414 & 7 \\
\hline WVE15652-8 & 28127 WVE15652 & $7 / 17 / 08$ 14:50 & 291.2589 & 8 \\
\hline WVE15652-9 & 28272 WVE15652 & 7/18/08 15:40 & 291.2801 & 9 \\
\hline WVE15652-10 & \begin{tabular}{l|l|l|}
28404 & WVE15652 \\
\end{tabular} & 7/21/08 11:28 & 291.2997 & 10 \\
\hline WVE15652-11 & \begin{tabular}{l|l|l|}
28752 & WVE15652 \\
\end{tabular} & 7/25/08 13:31 & 291.3289 & 11 \\
\hline WVE15652-12 & 29267 WVE15652 & 7/30/08 12:40 & 291.3543 & 12 \\
\hline WVE15652-13 & \begin{tabular}{l|l|l|}
29666 & WVE15652 \\
\end{tabular} & 8/7/08 19:40 & 291.381 & 13 \\
\hline WVE15652-14 & 29906 WVE15652 & 8/14/08 16:26 & 291.3992 & 14 \\
\hline WVE15652-15 & \begin{tabular}{l|l|}
30076 & WVE15652 \\
\end{tabular} & $8 / 21 / 08$ 10:35 & 291.4113 & 15 \\
\hline WVE15652-16 & \begin{tabular}{l|l|l}
30331 & WVE15652 \\
\end{tabular} & $8 / 28 / 08$ 10:12 & 291.4374 & 16 \\
\hline WVE15652-17 & 30553|WVE15652 & 9/3/08 16:36 & 291.4562 & 17 \\
\hline
\end{tabular}




\begin{tabular}{|c|c|c|c|c|}
\hline WVE15653-1 & 27745|WVE15653 & 7/11/08 18:45 & 288.2258 & 1 \\
\hline WVE15653-2 & 27782 $\mid$ WVE15653 & 7/12/08 13:10 & 288.2186 & 2 \\
\hline WVE15653-3 & 27815 WVE15653 & 7/13/08 15:08 & 288.2125 & 3 \\
\hline WVE15653-4 & \begin{tabular}{l|l|}
27946 & WVE15653
\end{tabular} & $7 / 14 / 08$ 14:33 & 288.2253 & 4 \\
\hline WVE15653-5 & 28000|WVE15653 & 7/15/08 13:35 & 287.4218 & 5 \\
\hline WVE15653-6 & 28031 $\mid$ WVE15653 & 7/15/08 19:28 & 287.4291 & 6 \\
\hline WVE15653-7 & 28099 $\mid$ WVE15653 & $7 / 16 / 08$ 19:40 & 287.4517 & 7 \\
\hline WVE15653-8 & 28128 $\mid$ WVE15653 & 7/17/08 14:51 & 287.4697 & 8 \\
\hline WVE15653-9 & 28273 $\mid$ WVE15653 & 7/18/08 15:41 & 287.4871 & 9 \\
\hline WVE15653-10 & $28405 \mid$ WVE15653 & $7 / 21 / 08$ 11:29 & 287.5174 & 10 \\
\hline WVE15653-11 & 28753 $\mid$ WVE15653 & $7 / 25 / 08$ 13:32 & 287.5589 & 11 \\
\hline WVE15653-12 & 29268|WVE15653 & $7 / 30 / 08$ 12:41 & 287.5991 & 12 \\
\hline WVE15653-13 & 29667 WVE15653 & 8/7/08 19:41 & 287.6239 & 13 \\
\hline WVE15653-14 & 29907 $\mid$ WVE15653 & $8 / 14 / 08$ 16:27 & 287.6349 & 14 \\
\hline WVE15653-15 & \begin{tabular}{l|l|l}
30077 & WVE15653
\end{tabular} & $8 / 21 / 08$ 10:36 & 287.6568 & 15 \\
\hline WVE15653-16 & \begin{tabular}{l|l|l}
30332 & WVE15653
\end{tabular} & 8/28/08 10:12 & 287.6779 & 16 \\
\hline WVE15653-17 & 30554 WVE15653 & 9/3/08 16:37 & 287.6974 & 17 \\
\hline WVE15654-1 & \begin{tabular}{l|l|l|}
27746 & WVE15654 \\
\end{tabular} & 7/11/08 18:46 & 282.9813 & 1 \\
\hline WVE15654-2 & \begin{tabular}{|l|l|}
27783 & WVE15654 \\
\end{tabular} & 7/12/08 13:11 & 282.9795 & 2 \\
\hline WVE15654-3 & \begin{tabular}{|l|l|}
27816 & WVE15654 \\
\end{tabular} & 7/13/08 15:09 & 282.9822 & 3 \\
\hline WVE15654-4 & \begin{tabular}{l|l|l|}
27947 & WVE15654 \\
\end{tabular} & 7/14/08 14:34 & 282.9891 & 4 \\
\hline WVE15654-5 & \begin{tabular}{l|l|l|}
28001 & WVE15654 \\
\end{tabular} & 7/15/08 13:36 & 282.1559 & 5 \\
\hline WVE15654-6 & \begin{tabular}{|l|l|}
28032 & WVE15654 \\
\end{tabular} & $7 / 15 / 08$ 19:29 & 282.1726 & 6 \\
\hline WVE15654-7 & \begin{tabular}{|l|l|}
28100 & WVE15654 \\
\end{tabular} & 7/16/08 19:41 & 282.2002 & 7 \\
\hline WVE15654-8 & 28129 $\mid$ WVE15654 & $7 / 17 / 08$ 14:52 & 282.2168 & 8 \\
\hline WVE15654-9 & 28274 WVE15654 & 7/18/08 15:42 & 282.2335 & 9 \\
\hline WVE15654-10 & \begin{tabular}{|l|l|}
28406 & WVE15654 \\
\end{tabular} & $7 / 21 / 08$ 11:30 & 282.268 & 10 \\
\hline WVE15654-11 & 28754 WVE15654 & 7/25/08 13:32 & 282.3054 & 11 \\
\hline WVE15654-12 & \begin{tabular}{l|l|l|}
29269 & WVE15654 \\
\end{tabular} & 7/30/08 12:41 & 282.3441 & 12 \\
\hline WVE15654-13 & 29668|WVE15654 & 8/7/08 19:42 & 282.3787 & 13 \\
\hline WVE15654-14 & \begin{tabular}{|l|l|}
29908 & WVE15654 \\
\end{tabular} & 8/14/08 16:28 & 282.4009 & 14 \\
\hline WVE15654-15 & \begin{tabular}{l|l|l|}
30078 & WVE15654 \\
\end{tabular} & 8/21/08 10:37 & 282.4236 & 15 \\
\hline WVE15654-16 & 30333 WVE15654 & $8 / 28 / 08$ 10:13 & 282.4454 & 16 \\
\hline WVE15654-17 & 30555 $\mid$ WVE15654 & 9/3/08 16:38 & 282.4655 & 17 \\
\hline WVE15655-1 & 27747 WVE15655 & 7/11/08 18:47 & 283.1308 & 1 \\
\hline WVE15655-2 & \begin{tabular}{|l|l|l|}
27784 & WVE15655 \\
\end{tabular} & 7/12/08 13:11 & 283.1203 & 2 \\
\hline WVE15655-3 & \begin{tabular}{|l|l|}
27817 & WVE15655 \\
\end{tabular} & 7/13/08 15:10 & 283.1145 & 3 \\
\hline WVE15655-4 & 27948 $\mid$ WVE15655 & $7 / 14 / 08$ 14:35 & 283.1195 & 4 \\
\hline WVE15655-5 & 28002 WVE15655 & 7/15/08 13:37 & 282.2939 & 5 \\
\hline WVE15655-6 & \begin{tabular}{|l|l|}
28033 & WVE15655 \\
\end{tabular} & 7/15/08 19:30 & 282.2963 & 6 \\
\hline WVE15655-7 & \begin{tabular}{l|l|l|}
28101 & WVE15655
\end{tabular} & 7/16/08 19:42 & 282.3219 & 7 \\
\hline WVE15655-8 & 28130 $\mid$ WVE15655 & 7/17/08 14:52 & 282.3366 & 8 \\
\hline WVE15655-9 & 28275 WVE15655 & 7/18/08 15:42 & 282.3539 & 9 \\
\hline WVE15655-10 & \begin{tabular}{|l|l|l|}
28407 & WVE15655 \\
\end{tabular} & 7/21/08 11:30 & 282.3844 & 10 \\
\hline WVE15655-11 & 28755 WVE15655 & 7/25/08 13:33 & 282.4244 & 11 \\
\hline WVE15655-12 & \begin{tabular}{|l|l|}
29270 & WVE15655 \\
\end{tabular} & 7/30/08 12:42 & 282.4638 & 12 \\
\hline WVE15655-13 & 29669 WVE15655 & 8/7/08 19:42 & 282.4942 & 13 \\
\hline WVE15655-14 & 29909 WVE15655 & 8/14/08 16:29 & 282.5218 & 14 \\
\hline WVE15655-15 & \begin{tabular}{l|l|l}
30079 & WVE15655 \\
\end{tabular} & 8/21/08 10:37 & 282.5407 & 15 \\
\hline
\end{tabular}




\begin{tabular}{|c|c|c|c|c|}
\hline WVE15655-16 & 30334 $\mid$ WVE15655 & 8/28/08 10:14 & 282.5679 & 16 \\
\hline WVE15655-17 & 30556|WVE15655 & 9/3/08 16:39 & 282.586 & 17 \\
\hline WVE15656-1 & \begin{tabular}{|l|l|}
27748 & WVE15656
\end{tabular} & 7/11/08 18:48 & 278.685 & 1 \\
\hline WVE15656-2 & \begin{tabular}{l|l|l}
27785 & WVE15656 \\
\end{tabular} & 7/12/08 13:12 & 278.6789 & 2 \\
\hline WVE15656-3 & 27818|WVE15656 & 7/13/08 15:11 & 278.6886 & 3 \\
\hline WVE15656-4 & 27949 WVE15656 & 7/14/08 14:36 & 278.6888 & 4 \\
\hline WVE15656-5 & 28003 WVE15656 & 7/15/08 13:38 & 277.8463 & 5 \\
\hline WVE15656-6 & \begin{tabular}{l|l|l|}
28034 & WVE15656 \\
\end{tabular} & 7/15/08 19:32 & 277.8526 & 6 \\
\hline WVE15656-7 & \begin{tabular}{l|l|l|}
28102 & WVE15656 \\
\end{tabular} & 7/16/08 19:43 & 277.8745 & 7 \\
\hline WVE15656-8 & \begin{tabular}{l|l|l|}
28131 & WVE15656 \\
\end{tabular} & $7 / 17 / 08$ 14:53 & 277.8918 & 8 \\
\hline WVE15656-9 & \begin{tabular}{l|l|l}
28276 & WVE15656 \\
\end{tabular} & 7/18/08 15:43 & 277.9139 & 9 \\
\hline WVE15656-10 & \begin{tabular}{l|l|l|}
28408 & WVE15656
\end{tabular} & 7/21/08 11:31 & 277.949 & 10 \\
\hline WVE15656-11 & 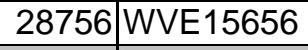 & 7/25/08 13:34 & 277.9963 & 11 \\
\hline WVE15656-12 & \begin{tabular}{l|l}
29271 & WVE15656 \\
\end{tabular} & $7 / 30 / 08$ 12:43 & 278.0378 & 12 \\
\hline WVE15656-13 & 29670 WVE15656 & 8/7/08 19:43 & 278.0642 & 13 \\
\hline WVE15656-14 & \begin{tabular}{|l|l|}
29910 & WVE15656 \\
\end{tabular} & $8 / 14 / 08$ 16:29 & 278.0846 & 14 \\
\hline WVE15656-15 & 30080 WVE15656 & 8/21/08 10:38 & 278.107 & 15 \\
\hline WVE15656-16 & 30335 WVE15656 & 8/28/08 10:14 & 278.1349 & 16 \\
\hline WVE15656-17 & \begin{tabular}{l|l|l|}
30557 & WVE15656 \\
\end{tabular} & 9/3/08 16:40 & 278.1548 & 17 \\
\hline WVE15657-1 & 27739 WVE15657 & 7/11/08 18:39 & 278.9541 & 1 \\
\hline WVE15657-2 & \begin{tabular}{l|l|l|}
27786 & WVE15657 \\
\end{tabular} & 7/12/08 13:13 & 278.9526 & 2 \\
\hline WVE15657-3 & 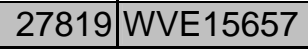 & 7/13/08 15:12 & 278.9994 & 3 \\
\hline WVE15657-4 & 27950 WVE15657 & 7/14/08 14:37 & 279.0012 & 4 \\
\hline WVE15657-5 & 28004 WVE15657 & 7/15/08 13:39 & 278.1764 & 5 \\
\hline WVE15657-6 & \begin{tabular}{l|l|l|}
28035 & WVE15657 \\
\end{tabular} & 7/15/08 19:32 & 278.1923 & 6 \\
\hline WVE15657-7 & 28093 $\mid$ WVE15657 & 7/16/08 19:33 & 278.2021 & 7 \\
\hline WVE15657-8 & \begin{tabular}{l|l|l|}
28132 & WVE15657 \\
\end{tabular} & 7/17/08 14:54 & 278.2225 & 8 \\
\hline WVE15657-9 & 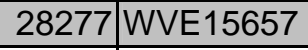 & 7/18/08 15:44 & 278.228 & 9 \\
\hline WVE15657-10 & 28409 WVE15657 & 7/21/08 11:32 & 278.2474 & 10 \\
\hline WVE15657-11 & \begin{tabular}{l|l|}
28757 & WVE15657 \\
\end{tabular} & 7/25/08 13:35 & 278.2737 & 11 \\
\hline WVE15657-12 & \begin{tabular}{l|l|l|}
29272 & WVE15657 \\
\end{tabular} & $7 / 30 / 08$ 12:43 & 278.3425 & 12 \\
\hline WVE15657-13 & \begin{tabular}{|l|l|}
29671 & WVE15657 \\
\end{tabular} & 8/7/08 19:44 & 278.3615 & 13 \\
\hline WVE15657-14 & \begin{tabular}{l|l|l|}
29911 & WVE15657 \\
\end{tabular} & 8/14/08 16:30 & 278.3878 & 14 \\
\hline WVE15657-15 & \begin{tabular}{|l|l|}
30081 & WVE15657 \\
\end{tabular} & $8 / 21 / 08$ 10:39 & 278.3986 & 15 \\
\hline WVE15657-16 & 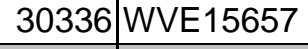 & 8/28/08 10:15 & 278.408 & 16 \\
\hline WVE15657-17 & \begin{tabular}{l|l|}
30558 & WVE15657 \\
\end{tabular} & 9/3/08 16:40 & 278.4312 & 17 \\
\hline WVE15658-1 & 27740 WVE15658 & 7/11/08 18:40 & 279.8038 & 1 \\
\hline WVE15658-2 & \begin{tabular}{l|l|}
27787 & WVE15658 \\
\end{tabular} & 7/12/08 13:14 & 279.7987 & 2 \\
\hline WVE15658-3 & 27820 WVE15658 & 7/13/08 15:12 & 279.7998 & 3 \\
\hline WVE15658-4 & 27951 WVE15658 & 7/14/08 14:38 & 279.8003 & 4 \\
\hline WVE15658-5 & 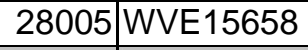 & 7/15/08 13:40 & 278.9259 & 5 \\
\hline WVE15658-6 & \begin{tabular}{|l|l|}
28036 & WVE15658 \\
\end{tabular} & 7/15/08 19:33 & 278.929 & 6 \\
\hline WVE15658-7 & $28094 \mid$ WVE15658 & 7/16/08 19:35 & 278.9539 & 7 \\
\hline WVE15658-8 & 28133 WVE15658 & $7 / 17 / 08$ 14:55 & 278.9723 & 8 \\
\hline WVE15658-9 & \begin{tabular}{l|l|l|}
28278 & WVE15658 \\
\end{tabular} & $7 / 18 / 08$ 15:45 & 278.9898 & 9 \\
\hline WVE15658-10 & 28410 WVE15658 & 7/21/08 11:32 & 279.0174 & 10 \\
\hline WVE15658-11 & \begin{tabular}{l|l|}
28758 & WVE15658 \\
\end{tabular} & $7 / 25 / 08$ 13:36 & 279.0566 & 11 \\
\hline WVE15658-12 & 29273 WVE15658 & 7/30/08 12:44 & 279.1054 & 12 \\
\hline WVE15658-13 & \begin{tabular}{l|l|l|}
29672 & WVE15658 \\
\end{tabular} & 8/7/08 19:45 & 279.1226 & 13 \\
\hline
\end{tabular}




\begin{tabular}{|c|c|c|c|c|}
\hline WVE15658-14 & 29912 $\mid$ WVE15658 & 8/14/08 16:31 & 279.1464 & 14 \\
\hline WVE15658-15 & \begin{tabular}{l|l|l|}
30082 & WVE15658
\end{tabular} & $8 / 21 / 08$ 10:39 & 279.1653 & 15 \\
\hline WVE15658-16 & \begin{tabular}{l|l|l}
30337 & WVE15658 \\
\end{tabular} & 8/28/08 10:16 & 279.1779 & 16 \\
\hline WVE15658-17 & 30559 $\mid$ WVE15658 & 9/3/08 16:41 & 279.1981 & 17 \\
\hline WVE15659-1 & \begin{tabular}{l|l|}
27741 & WVE15659
\end{tabular} & 7/11/08 18:41 & 281.6841 & 1 \\
\hline WVE15659-2 & 27788 $\mid$ WVE15659 & $7 / 12 / 08$ 13:14 & 281.6766 & 2 \\
\hline WVE15659-3 & 27821 $\mid$ WVE15659 & 7/13/08 15:13 & 281.6758 & 3 \\
\hline WVE15659-4 & \begin{tabular}{l|l}
27952 & WVE15659
\end{tabular} & $7 / 14 / 08$ 14:39 & 281.6798 & 4 \\
\hline WVE15659-5 & \begin{tabular}{l|l|l|}
28006 & WVE15659 \\
\end{tabular} & $7 / 15 / 08$ 13:40 & 280.8491 & 5 \\
\hline WVE15659-6 & 28037 $\mid$ WVE15659 & 7/15/08 19:34 & 280.8553 & 6 \\
\hline WVE15659-7 & \begin{tabular}{|l|l|}
28095 & WVE15659 \\
\end{tabular} & 7/16/08 19:36 & 280.8787 & 7 \\
\hline WVE15659-8 & \begin{tabular}{l|l}
28134 & WVE15659
\end{tabular} & $7 / 17 / 0814: 56$ & 280.8939 & 8 \\
\hline WVE15659-9 & \begin{tabular}{l|l|l}
28279 & WVE15659
\end{tabular} & $7 / 18 / 08$ 15:46 & 280.9145 & 9 \\
\hline WVE15659-10 & \begin{tabular}{l|l|l|}
28411 & WVE15659
\end{tabular} & $7 / 21 / 08$ 11:33 & 280.9487 & 10 \\
\hline WVE15659-11 & 28759 WVE15659 & 7/25/08 13:36 & 280.9882 & 11 \\
\hline WVE15659-12 & \begin{tabular}{l|l|l|}
29274 & WVE15659
\end{tabular} & $7 / 30 / 08$ 12:45 & 281.0438 & 12 \\
\hline WVE15659-13 & 29673|WVE15659 & 8/7/08 19:46 & 281.0642 & 13 \\
\hline WVE15659-14 & \begin{tabular}{l|l|}
29913 & WVE15659
\end{tabular} & 8/14/08 16:31 & 281.087 & 14 \\
\hline WVE15659-15 & \begin{tabular}{l|l|l}
30083 & WVE15659 \\
\end{tabular} & $8 / 21 / 08$ 10:40 & 281.1077 & 15 \\
\hline WVE15659-16 & 30338 $\mid$ WVE15659 & 8/28/08 10:17 & 281.1287 & 16 \\
\hline WVE15659-17 & 30560 $\mid$ WVE15659 & 9/3/08 16:42 & 281.1471 & 17 \\
\hline WVE15660-1 & \begin{tabular}{l|l|}
27742 & WVE15660 \\
\end{tabular} & $7 / 11 / 08$ 18:42 & 282.6451 & 1 \\
\hline WVE15660-2 & 27789 WVE15660 & $7 / 12 / 08$ 13:15 & 282.6393 & 2 \\
\hline WVE15660-3 & \begin{tabular}{l|l|l}
27822 & WVE15660
\end{tabular} & $7 / 13 / 08$ 15:14 & 282.6403 & 3 \\
\hline WVE15660-4 & 27953 WVE15660 & $7 / 14 / 08$ 14:40 & 282.6426 & 4 \\
\hline WVE15660-5 & 28007|WVE15660 & 7/15/08 13:41 & 281.8397 & 5 \\
\hline WVE15660-6 & 28038 WVE15660 & 7/15/08 19:35 & 281.848 & 6 \\
\hline WVE15660-7 & \begin{tabular}{l|l|l|}
28096 & WVE15660
\end{tabular} & 7/16/08 19:37 & 281.8684 & 7 \\
\hline WVE15660-8 & \begin{tabular}{l|l|l}
28135 & WVE15660
\end{tabular} & 7/17/08 14:57 & 281.8891 & 8 \\
\hline WVE15660-9 & \begin{tabular}{l|l|}
28280 & WVE15660
\end{tabular} & 7/18/08 15:47 & 281.91 & 9 \\
\hline WVE15660-10 & \begin{tabular}{l|l|}
28412 & WVE15660
\end{tabular} & $7 / 21 / 08$ 11:34 & 281.9493 & 10 \\
\hline WVE15660-11 & \begin{tabular}{l|l|l|}
28760 & WVE15660
\end{tabular} & $7 / 25 / 08$ 13:37 & 281.978 & 11 \\
\hline WVE15660-12 & 29275|WVE15660 & $7 / 30 / 08$ 12:45 & 282.0214 & 12 \\
\hline WVE15660-13 & 29674 WVE15660 & 8/7/08 19:47 & 282.0575 & 13 \\
\hline WVE15660-14 & $\begin{array}{l}29914 \mid \text { WVE15660 } \\
\end{array}$ & 8/14/08 16:32 & 282.0791 & 14 \\
\hline WVE15660-15 & 30084|WVE15660 & $8 / 21 / 08$ 10:41 & 282.101 & 15 \\
\hline WVE15660-16 & \begin{tabular}{l|l|l|}
30339 & WVE15660 \\
\end{tabular} & $8 / 28 / 08$ 10:17 & 282.1277 & 16 \\
\hline WVE15660-17 & 30561|WVE15660 & 9/3/08 16:43 & 282.151 & 17 \\
\hline WVE15661-1 & \begin{tabular}{l|l|}
27743 & WVE15661 \\
\end{tabular} & $7 / 11 / 08$ 18:43 & 280.7273 & 1 \\
\hline WVE15661-2 & \begin{tabular}{l|l|l}
27790 & WVE15661 \\
\end{tabular} & $7 / 12 / 08$ 13:16 & 280.7192 & 2 \\
\hline WVE15661-3 & 27823 $\mid$ WVE15661 & 7/13/08 15:15 & 280.7234 & 3 \\
\hline WVE15661-4 & \begin{tabular}{l|l}
27954 & WVE15661
\end{tabular} & $7 / 14 / 0814: 40$ & 280.7245 & 4 \\
\hline WVE15661-5 & \begin{tabular}{l|l|}
28008 & WVE15661
\end{tabular} & 7/15/08 13:42 & 279.8041 & 5 \\
\hline WVE15661-6 & 28039 WVE15661 & 7/15/08 19:35 & 279.8105 & 6 \\
\hline WVE15661-7 & \begin{tabular}{l|l|}
28097 & WVE15661
\end{tabular} & 7/16/08 19:38 & 279.835 & 7 \\
\hline WVE15661-8 & \begin{tabular}{l|l|l}
28136 & WVE15661
\end{tabular} & $7 / 17 / 08$ 14:57 & 279.85 & 8 \\
\hline WVE15661-9 & \begin{tabular}{l|l|}
28281 & WVE15661 \\
\end{tabular} & 7/18/08 15:48 & 279.8711 & 9 \\
\hline WVE15661-10 & \begin{tabular}{l|l|}
28413 & WVE15661
\end{tabular} & $7 / 21 / 08$ 11:35 & 279.9106 & 10 \\
\hline WVE15661-11 & \begin{tabular}{l|l|}
28761 & WVE15661
\end{tabular} & 7/25/08 13:38 & 279.9572 & 11 \\
\hline
\end{tabular}




\begin{tabular}{|c|c|c|c|c|}
\hline WVE15661-12 & \begin{tabular}{l|l|l}
29276 & WVE15661 \\
\end{tabular} & $7 / 30 / 08$ 12:46 & 279.9932 & 12 \\
\hline WVE15661-13 & \begin{tabular}{l|l|}
29675 & WVE15661
\end{tabular} & 8/7/08 19:48 & 280.029 & 13 \\
\hline WVE15661-14 & 29915 $\mid$ WVE15661 & 8/14/08 16:33 & 280.0441 & 14 \\
\hline WVE15661-15 & \begin{tabular}{l|l|}
30085 & WVE15661
\end{tabular} & $8 / 21 / 08$ 10:42 & 280.0641 & 15 \\
\hline WVE15661-16 & 30340|WVE15661 & 8/28/08 10:18 & 280.0834 & 16 \\
\hline WVE15661-17 & \begin{tabular}{l|l|}
30562 & WVE15661
\end{tabular} & 9/3/08 16:43 & 280.1018 & 17 \\
\hline WVE15831-1 & $26649 \mid$ WVE15831 & 7/2/08 13:31 & 300.563 & 1 \\
\hline WVE15831-2 & \begin{tabular}{l|l|}
26756 & WVE15831
\end{tabular} & $7 / 3 / 089: 25$ & 300.5567 & 2 \\
\hline WVE15831-3 & \begin{tabular}{|l|l|}
26860 & WVE15831 \\
\end{tabular} & $7 / 3 / 08$ 14:08 & 300.5578 & 3 \\
\hline WVE15831-4 & \begin{tabular}{|l|l|}
26965 & WVE15831
\end{tabular} & 7/7/08 9:00 & 300.552 & 4 \\
\hline WVE15831-5 & 27134 $\mid$ WVE15831 & 7/8/08 9:34 & 300.5518 & 5 \\
\hline WVE15831-6 & \begin{tabular}{|l|l|}
27355 & WVE15831 \\
\end{tabular} & 7/9/08 9:45 & 300.551 & 6 \\
\hline WVE15831-7 & 27486|WVE15831 & $7 / 10 / 08$ 15:04 & 300.5494 & 7 \\
\hline WVE15831-8 & \begin{tabular}{|l|l|}
27596 & WVE15831 \\
\end{tabular} & 7/11/08 9:39 & 300.5513 & 8 \\
\hline WVE15831-9 & 27837 $\mid$ WVE15831 & $7 / 14 / 08$ 12:14 & 300.5515 & 9 \\
\hline WVE15831-10 & \begin{tabular}{l|l|}
28157 & WVE15831 \\
\end{tabular} & 7/18/08 13:19 & 300.5537 & 10 \\
\hline WVE15831-11 & 28764|WVE15831 & 7/25/08 13:39 & 300.5528 & 11 \\
\hline WVE15831-12 & 29159|WVE15831 & 7/30/08 11:10 & 300.5535 & 12 \\
\hline WVE15831-13 & 29557 $\mid$ WVE15831 & 8/7/08 17:22 & 300.5567 & 13 \\
\hline WVE15831-14 & \begin{tabular}{|l|l|}
29923 & WVE15831 \\
\end{tabular} & 8/14/08 16:38 & 300.5605 & 14 \\
\hline WVE15831-15 & 30094|WVE15831 & $8 / 21 / 08$ 10:50 & 300.5601 & 15 \\
\hline WVE15831-16 & \begin{tabular}{l|l|l}
30349 & WVE15831 \\
\end{tabular} & $8 / 28 / 08$ 10:26 & 300.5619 & 16 \\
\hline WVE15831-17 & \begin{tabular}{l|l|l}
30570 & WVE15831 \\
\end{tabular} & 9/3/08 16:51 & 300.5654 & 17 \\
\hline WVE15832-1 & 26650|WVE15832 & 7/2/08 13:32 & 302.0723 & 1 \\
\hline WVE15832-2 & 26757|WVE15832 & $7 / 3 / 089: 26$ & 302.07 & 2 \\
\hline WVE15832-3 & 26861 WVE15832 & 7/3/08 14:09 & 302.0698 & 3 \\
\hline WVE15832-4 & 26966|WVE15832 & 7/7/08 9:00 & 302.0657 & 4 \\
\hline WVE15832-5 & 27135|WVE15832 & 7/8/08 9:35 & 302.0641 & 5 \\
\hline WVE15832-6 & \begin{tabular}{|l|l|} 
27356 & WVE15832 \\
\end{tabular} & 7/9/08 9:46 & 302.0648 & 6 \\
\hline WVE15832-7 & \begin{tabular}{l|l|}
27487 & WVE15832
\end{tabular} & 7/10/08 15:05 & 302.064 & 7 \\
\hline WVE15832-8 & $27595 \mid$ WVE15832 & 7/11/08 9:38 & 302.0651 & 8 \\
\hline WVE15832-9 & 27838|WVE15832 & $7 / 14 / 08$ 12:15 & 302.0632 & 9 \\
\hline WVE15832-10 & 28158|WVE15832 & 7/18/08 13:20 & 302.0634 & 10 \\
\hline WVE15832-11 & \begin{tabular}{l|l|}
28765 & WVE15832
\end{tabular} & 7/25/08 13:40 & 302.0638 & 11 \\
\hline WVE15832-12 & \begin{tabular}{|l|l|}
29160 & WVE15832 \\
\end{tabular} & 7/30/08 11:11 & 302.0633 & 12 \\
\hline WVE15832-13 & 29558|WVE15832 & 8/7/08 17:23 & 302.067 & 13 \\
\hline WVE15832-14 & 29924|WVE15832 & 8/14/08 16:39 & 302.0704 & 14 \\
\hline WVE15832-15 & 30095|WVE15832 & $8 / 21 / 08$ 10:51 & 302.0717 & 15 \\
\hline WVE15832-16 & 30350 $\mid$ WVE15832 & $8 / 28 / 08$ 10:27 & 302.0749 & 16 \\
\hline WVE15832-17 & 30571 $\mid$ WVE15832 & 9/3/08 16:52 & 302.0809 & 17 \\
\hline WVE15833-1 & $26651 \mid$ WVE15833 & $7 / 2 / 08$ 13:33 & 301.3339 & 1 \\
\hline WVE15833-2 & 26758|WVE15833 & 7/3/08 9:27 & 301.3318 & 2 \\
\hline WVE15833-3 & 26862|WVE15833 & $7 / 3 / 08$ 14:10 & 301.3292 & 3 \\
\hline WVE15833-4 & \begin{tabular}{l|l|l|}
26967 & WVE15833
\end{tabular} & 7/7/08 9:01 & 301.3291 & 4 \\
\hline WVE15833-5 & \begin{tabular}{l|l|l|}
27136 & WVE15833
\end{tabular} & $7 / 8 / 089: 36$ & 301.3265 & 5 \\
\hline WVE15833-6 & \begin{tabular}{|l|l|}
27357 & WVE15833 \\
\end{tabular} & 7/9/08 9:47 & 301.3263 & 6 \\
\hline WVE15833-7 & 27488|WVE15833 & 7/10/08 15:06 & 301.3255 & 7 \\
\hline WVE15833-8 & 27594 WVE15833 & 7/11/08 9:38 & 301.3251 & 8 \\
\hline WVE15833-9 & \begin{tabular}{l|l|}
27839 & WVE15833 \\
\end{tabular} & $7 / 14 / 08$ 12:16 & 301.3242 & 9 \\
\hline
\end{tabular}




\begin{tabular}{|c|c|c|c|c|}
\hline WVE15833-10 & 28159 WVE15833 & 7/18/08 13:21 & 301.3271 & 10 \\
\hline WVE15833-11 & \begin{tabular}{l|l|l}
28766 & WVE15833
\end{tabular} & $7 / 25 / 08$ 13:41 & 301.326 & 11 \\
\hline WVE15833-12 & \begin{tabular}{l|l|}
29161 & WVE15833
\end{tabular} & 7/30/08 11:12 & 301.3273 & 12 \\
\hline WVE15833-13 & \begin{tabular}{l|l|}
29559 & WVE15833 \\
\end{tabular} & $8 / 7 / 0817: 23$ & 301.3282 & 13 \\
\hline WVE15833-14 & $29925 \mid$ WVE15833 & $8 / 14 / 08$ 16:40 & 301.3327 & 14 \\
\hline WVE15833-15 & 30096|WVE15833 & $8 / 21 / 08$ 10:52 & 301.3297 & 15 \\
\hline WVE15833-16 & \begin{tabular}{l|l|l}
30351 & WVE15833 \\
\end{tabular} & $8 / 28 / 08$ 10:28 & 301.3361 & 16 \\
\hline WVE15833-17 & \begin{tabular}{l|l}
30572 & WVE15833
\end{tabular} & 9/3/08 16:52 & 301.3441 & 17 \\
\hline WVE15834-1 & 26652 |WVE15834 & 7/2/08 13:34 & 302.0524 & 1 \\
\hline WVE15834-2 & 26759 WVE15834 & 7/3/08 9:27 & 302.0478 & 2 \\
\hline WVE15834-3 & \begin{tabular}{l|l|l}
26863 & WVE15834 \\
\end{tabular} & $7 / 3 / 08$ 14:10 & 302.0461 & 3 \\
\hline WVE15834-4 & \begin{tabular}{l|l|}
26968 & WVE15834 \\
\end{tabular} & 7/7/08 9:02 & 302.0418 & 4 \\
\hline WVE15834-5 & \begin{tabular}{l|l|l|}
27137 & WVE15834 \\
\end{tabular} & 7/8/08 9:37 & 302.0421 & 5 \\
\hline WVE15834-6 & \begin{tabular}{l|l|l|}
27358 & WVE15834
\end{tabular} & 7/9/08 9:48 & 302.0417 & 6 \\
\hline WVE15834-7 & \begin{tabular}{l|l|l|}
27489 & WVE15834 \\
\end{tabular} & 7/10/08 15:07 & 302.0414 & 7 \\
\hline WVE15834-8 & \begin{tabular}{l|l|l}
27593 & WVE15834
\end{tabular} & 7/11/08 9:37 & 302.0418 & 8 \\
\hline WVE15834-9 & \begin{tabular}{l|l|}
27840 & WVE15834 \\
\end{tabular} & $7 / 14 / 08$ 12:17 & 302.0445 & 9 \\
\hline WVE15834-10 & \begin{tabular}{l|l|}
28160 & WVE15834
\end{tabular} & $7 / 18 / 08$ 13:22 & 302.0455 & 10 \\
\hline WVE15834-11 & \begin{tabular}{l|l|}
28767 & WVE15834 \\
\end{tabular} & $7 / 25 / 08$ 13:42 & 302.0453 & 11 \\
\hline WVE15834-12 & \begin{tabular}{l|l|}
29162 & WVE15834
\end{tabular} & $7 / 30 / 08$ 11:13 & 302.0442 & 12 \\
\hline WVE15834-13 & \begin{tabular}{l|l|}
29560 & WVE15834
\end{tabular} & 8/7/08 17:24 & 302.0448 & 13 \\
\hline WVE15834-14 & \begin{tabular}{l|l|}
29926 & WVE15834
\end{tabular} & $8 / 14 / 08$ 16:40 & 302.046 & 14 \\
\hline WVE15834-15 & \begin{tabular}{l|l|}
30097 & WVE15834 \\
\end{tabular} & $8 / 21 / 08$ 10:52 & 302.047 & 15 \\
\hline WVE15834-16 & \begin{tabular}{l|l|l|}
30352 & WVE15834 \\
\end{tabular} & 8/28/08 10:28 & 302.053 & 16 \\
\hline WVE15834-17 & \begin{tabular}{l|l}
30573 & WVE15834
\end{tabular} & 9/3/08 16:53 & 302.0606 & 17 \\
\hline WVE15835-1 & $26653 \mid$ WVE15835 & 7/2/08 13:35 & 304.4663 & 1 \\
\hline WVE15835-2 & \begin{tabular}{l|l}
26760 & WVE15835
\end{tabular} & 7/3/08 9:28 & 304.4644 & 2 \\
\hline WVE15835-3 & 26864|WVE15835 & $7 / 3 / 08$ 14:11 & 304.4626 & 3 \\
\hline WVE15835-4 & 26969 WVE15835 & 7/7/08 9:03 & 304.4566 & 4 \\
\hline WVE15835-5 & \begin{tabular}{l|l|l|}
27138 & WVE15835 \\
\end{tabular} & 7/8/08 9:37 & 304.4542 & 5 \\
\hline WVE15835-6 & \begin{tabular}{l|l|l}
27359 & WVE15835 \\
\end{tabular} & 7/9/08 9:48 & 304.455 & 6 \\
\hline WVE15835-7 & \begin{tabular}{l|l|l|}
27490 & WVE15835
\end{tabular} & 7/10/08 15:08 & 304.4557 & 7 \\
\hline WVE15835-8 & \begin{tabular}{l|l|}
27597 & WVE15835
\end{tabular} & 7/11/08 9:40 & 304.4555 & 8 \\
\hline WVE15835-9 & \begin{tabular}{l|l|}
27841 & WVE15835
\end{tabular} & $7 / 14 / 08$ 12:18 & 304.4545 & 9 \\
\hline WVE15835-10 & \begin{tabular}{l|l|}
28161 & WVE15835 \\
\end{tabular} & 7/18/08 13:22 & 304.4563 & 10 \\
\hline WVE15835-11 & $28768 \mid$ WVE15835 & 7/25/08 13:42 & 304.4569 & 11 \\
\hline WVE15835-12 & \begin{tabular}{l|l|l|}
29163 & WVE15835 \\
\end{tabular} & 7/30/08 11:13 & 304.4557 & 12 \\
\hline WVE15835-13 & 29561|WVE15835 & $8 / 7 / 08$ 17:25 & 304.4596 & 13 \\
\hline WVE15835-14 & \begin{tabular}{l|l|l|}
29927 & WVE15835 \\
\end{tabular} & $8 / 14 / 08$ 16:41 & 304.459 & 14 \\
\hline WVE15835-15 & \begin{tabular}{l|l|l|}
30093 & WVE15835 \\
\end{tabular} & $8 / 21 / 08$ 10:49 & 304.4592 & 15 \\
\hline WVE15835-16 & \begin{tabular}{l|l|}
30348 & WVE15835 \\
\end{tabular} & $8 / 28 / 08$ 10:25 & 304.4606 & 16 \\
\hline WVE15835-17 & \begin{tabular}{l|l}
30574 & WVE15835
\end{tabular} & 9/3/08 16:54 & 304.4642 & 17 \\
\hline WVE15836-1 & \begin{tabular}{l|l|l|}
26654 & WVE15836
\end{tabular} & $7 / 2 / 08$ 13:36 & 302.9846 & 1 \\
\hline WVE15836-2 & \begin{tabular}{l|l|l|}
26761 & WVE15836
\end{tabular} & $7 / 3 / 089: 29$ & 302.9838 & 2 \\
\hline WVE15836-3 & \begin{tabular}{l|l|}
26865 & WVE15836 \\
\end{tabular} & 7/3/08 14:12 & 302.9829 & 3 \\
\hline WVE15836-4 & 26970|WVE15836 & 7/7/08 9:04 & 302.978 & 4 \\
\hline WVE15836-5 & 27203 WVE15836 & 7/8/08 14:31 & 302.9983 & 5 \\
\hline WVE15836-6 & \begin{tabular}{l|l|l}
27276 & WVE15836
\end{tabular} & $7 / 8 / 08$ 21:03 & 302.9915 & 6 \\
\hline WVE15836-7 & \begin{tabular}{l|l}
27360 & WVE15836
\end{tabular} & 7/9/08 9:49 & 302.9858 & 7 \\
\hline
\end{tabular}




\begin{tabular}{|c|c|c|c|c|}
\hline WVE15836-8 & 27491|WVE15836 & 7/10/08 15:09 & 302.9845 & 8 \\
\hline WVE15836-9 & \begin{tabular}{l|l|}
27598 & WVE15836
\end{tabular} & 7/11/08 9:41 & 302.9812 & 9 \\
\hline WVE15836-10 & \begin{tabular}{l|l|l|}
27842 & WVE15836
\end{tabular} & 7/14/08 12:19 & 302.9791 & 10 \\
\hline WVE15836-11 & \begin{tabular}{l|l}
28162 & WVE15836
\end{tabular} & 7/18/08 13:24 & 302.9793 & 11 \\
\hline WVE15836-12 & \begin{tabular}{|l|l|}
28769 & WVE15836
\end{tabular} & 7/25/08 13:43 & 302.9766 & 12 \\
\hline WVE15836-13 & 29164|WVE15836 & 7/30/08 11:14 & 302.9811 & 13 \\
\hline WVE15836-14 & \begin{tabular}{|l|l|}
29562 & WVE15836 \\
\end{tabular} & $8 / 7 / 08$ 17:26 & 302.9795 & 14 \\
\hline WVE15836-15 & 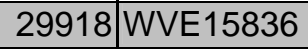 & $8 / 14 / 08$ 16:35 & 302.9838 & 15 \\
\hline WVE15836-16 & \begin{tabular}{|c|c|}
30088 & WVE15836 \\
\end{tabular} & $8 / 21 / 08$ 10:45 & 302.9833 & 16 \\
\hline WVE15836-17 & \begin{tabular}{l|l|l}
30343 & WVE15836 \\
\end{tabular} & 8/28/08 10:21 & 302.9877 & 17 \\
\hline WVE15836-18 & 30565|WVE15836 & 9/3/08 16:46 & 302.9868 & 18 \\
\hline WVE15837-1 & \begin{tabular}{l|l|l}
26655 & WVE15837
\end{tabular} & $7 / 2 / 08$ 13:37 & 298.0392 & 1 \\
\hline WVE15837-2 & \begin{tabular}{l|l|l}
26762 & WVE15837
\end{tabular} & 7/3/08 9:30 & 298.0355 & 2 \\
\hline WVE15837-3 & \begin{tabular}{l|l|}
26866 & WVE15837 \\
\end{tabular} & $7 / 3 / 08$ 14:12 & 298.0356 & 3 \\
\hline WVE15837-4 & \begin{tabular}{l|l|l|}
27011 & WVE15837 \\
\end{tabular} & 7/7/08 9:40 & 298.0348 & 4 \\
\hline WVE15837-5 & \begin{tabular}{l|l|}
27204 & WVE15837
\end{tabular} & $7 / 8 / 08$ 14:32 & 298.0455 & 5 \\
\hline WVE15837-6 & $27275 \mid$ WVE15837 & 7/8/08 21:02 & 298.0372 & 6 \\
\hline WVE15837-7 & \begin{tabular}{l|l|l}
27361 & WVE15837 \\
\end{tabular} & 7/9/08 9:50 & 298.0329 & 7 \\
\hline WVE15837-8 & \begin{tabular}{l|l|}
27492 & WVE15837 \\
\end{tabular} & 7/10/08 15:09 & 298.0301 & 8 \\
\hline WVE15837-9 & 27599 WVE15837 & 7/11/08 9:41 & 298.0274 & 9 \\
\hline WVE15837-10 & $27843 \mid$ WVE15837 & $7 / 14 / 08$ 12:20 & 298.0292 & 10 \\
\hline WVE15837-11 & \begin{tabular}{l|l|l|}
28163 & WVE15837 \\
\end{tabular} & 7/18/08 13:24 & 298.0257 & 11 \\
\hline WVE15837-12 & \begin{tabular}{l|l|l}
28770 & WVE15837 \\
\end{tabular} & $7 / 25 / 08$ 13:44 & 298.0218 & 12 \\
\hline WVE15837-13 & 29165 WVE15837 & 7/30/08 11:15 & 298.0248 & 13 \\
\hline WVE15837-14 & $29563 \mid$ WVE15837 & 8/7/08 17:27 & 298.0276 & 14 \\
\hline WVE15837-15 & 29919 WVE15837 & $8 / 14 / 08$ 16:35 & 298.0296 & 15 \\
\hline WVE15837-16 & 30089 WVE15837 & $8 / 21 / 08$ 10:46 & 298.0296 & 16 \\
\hline WVE15837-17 & \begin{tabular}{l|l|l|}
30344 & WVE15837 \\
\end{tabular} & $8 / 28 / 08$ 10:22 & 298.0318 & 17 \\
\hline WVE15837-18 & \begin{tabular}{l|l|l}
30566 & WVE15837 \\
\end{tabular} & 9/3/08 16:47 & 298.0359 & 18 \\
\hline WVE15838-1 & \begin{tabular}{l|l|l}
26656 & WVE15838 \\
\end{tabular} & $7 / 2 / 08$ 13:38 & 293.5489 & 1 \\
\hline WVE15838-2 & $26763 \mid$ WVE15838 & 7/3/08 9:31 & 293.5454 & 2 \\
\hline WVE15838-3 & \begin{tabular}{l|l|l|}
26867 & WVE15838 \\
\end{tabular} & 7/3/08 14:13 & 293.5452 & 3 \\
\hline WVE15838-4 & 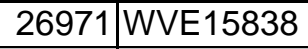 & 7/7/08 9:04 & 293.5398 & 4 \\
\hline WVE15838-5 & \begin{tabular}{|l|l|}
27205 & WVE15838 \\
\end{tabular} & 7/8/08 14:32 & 293.5531 & 5 \\
\hline WVE15838-6 & 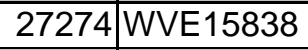 & $7 / 8 / 08$ 21:01 & 293.5466 & 6 \\
\hline WVE15838-7 & \begin{tabular}{l|l|}
27362 & WVE15838 \\
\end{tabular} & 7/9/08 9:51 & 293.5438 & 7 \\
\hline WVE15838-8 & 27493 $\mid$ WVE15838 & 7/10/08 15:10 & 293.5395 & 8 \\
\hline WVE15838-9 & \begin{tabular}{l|l|l|}
27600 & WVE15838 \\
\end{tabular} & 7/11/08 9:42 & 293.5372 & 9 \\
\hline WVE15838-10 & $27844 \mid$ WVE15838 & $7 / 14 / 08$ 12:21 & 293.5381 & 10 \\
\hline WVE15838-11 & \begin{tabular}{l|l|l|}
28164 & WVE15838 \\
\end{tabular} & $7 / 18 / 08$ 13:25 & 293.5343 & 11 \\
\hline WVE15838-12 & \begin{tabular}{l|l|l|}
28771 & WVE15838 \\
\end{tabular} & 7/25/08 13:44 & 293.5321 & 12 \\
\hline WVE15838-13 & \begin{tabular}{l|l|}
29166 & WVE15838 \\
\end{tabular} & $7 / 30 / 08$ 11:15 & 293.5361 & 13 \\
\hline WVE15838-14 & $29564 \mid$ WVE15838 & 8/7/08 17:28 & 293.5359 & 14 \\
\hline WVE15838-15 & 29920 WVE15838 & 8/14/08 16:36 & 293.5378 & 15 \\
\hline WVE15838-16 & \begin{tabular}{|l|l|}
30090 & WVE15838 \\
\end{tabular} & $8 / 21 / 08$ 10:47 & 293.5368 & 16 \\
\hline WVE15838-17 & \begin{tabular}{l|l|l|}
30345 & WVE15838 \\
\end{tabular} & $8 / 28 / 08$ 10:23 & 293.5365 & 17 \\
\hline WVE15838-18 & \begin{tabular}{l|l|l|}
30567 & WVE15838 \\
\end{tabular} & 9/3/08 16:48 & 293.5414 & 18 \\
\hline WVE15839-1 & \begin{tabular}{l|l|l|}
26657 & WVE15839 \\
\end{tabular} & 7/2/08 13:39 & 293.209 & 1 \\
\hline WVE15839-2 & \begin{tabular}{l|l|}
26764 & WVE15839 \\
\end{tabular} & 7/3/08 9:31 & 293.2041 & 2 \\
\hline
\end{tabular}




\begin{tabular}{|c|c|c|c|c|}
\hline WVE15839-3 & 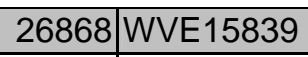 & $7 / 3 / 08$ 14:14 & 293.2015 & 3 \\
\hline WVE15839-4 & \begin{tabular}{l|l|l|}
26972 & WVE15839
\end{tabular} & 7/7/08 9:05 & 293.2016 & 4 \\
\hline WVE15839-5 & \begin{tabular}{|l|l|}
27206 & WVE15839 \\
\end{tabular} & $7 / 8 / 08$ 14:33 & 293.2192 & 5 \\
\hline WVE15839-6 & \begin{tabular}{l|l|}
27273 & WVE15839 \\
\end{tabular} & 7/8/08 21:00 & 293.2109 & 6 \\
\hline WVE15839-7 & \begin{tabular}{l|l|l}
27363 & WVE15839
\end{tabular} & 7/9/08 9:52 & 293.2079 & 7 \\
\hline WVE15839-8 & 27494|WVE15839 & 7/10/08 15:11 & 293.2048 & 8 \\
\hline WVE15839-9 & \begin{tabular}{l|l|l|}
27601 & WVE15839 \\
\end{tabular} & 7/11/08 9:43 & 293.2026 & 9 \\
\hline WVE15839-10 & \begin{tabular}{l|l|}
27845 & WVE15839
\end{tabular} & $7 / 14 / 08$ 12:22 & 293.2011 & 10 \\
\hline WVE15839-11 & \begin{tabular}{l|l|}
28165 & WVE15839 \\
\end{tabular} & $7 / 18 / 08$ 13:26 & 293.1996 & 11 \\
\hline WVE15839-12 & \begin{tabular}{l|l|}
28772 & WVE15839 \\
\end{tabular} & $7 / 25 / 08$ 13:45 & 293.2 & 12 \\
\hline WVE15839-13 & \begin{tabular}{l|l|}
29167 & WVE15839 \\
\end{tabular} & 7/30/08 11:16 & 293.2005 & 13 \\
\hline WVE15839-14 & \begin{tabular}{l|l|}
29565 & WVE15839
\end{tabular} & $8 / 7 / 08$ 17:30 & 293.203 & 14 \\
\hline WVE15839-15 & \begin{tabular}{l|l|l|}
29921 & WVE15839
\end{tabular} & 8/14/08 16:37 & 293.2048 & 15 \\
\hline WVE15839-16 & \begin{tabular}{l|l}
30091 & WVE15839
\end{tabular} & $8 / 21 / 08$ 10:47 & 293.2058 & 16 \\
\hline WVE15839-17 & \begin{tabular}{l|l|}
30346 & WVE15839 \\
\end{tabular} & $8 / 28 / 08$ 10:24 & 293.2108 & 17 \\
\hline WVE15839-18 & \begin{tabular}{l|l|}
30568 & WVE15839
\end{tabular} & 9/3/08 16:48 & 293.2158 & 18 \\
\hline WVE15840-1 & \begin{tabular}{l|l}
26658 & WVE15840
\end{tabular} & $7 / 2 / 08$ 13:39 & 302.0644 & 1 \\
\hline WVE15840-2 & \begin{tabular}{l|l|l|}
26765 & WVE15840
\end{tabular} & 7/3/08 9:32 & 302.0654 & 2 \\
\hline WVE15840-3 & \begin{tabular}{l|l|l}
26869 & WVE15840 \\
\end{tabular} & $7 / 3 / 08$ 14:15 & 302.0591 & 3 \\
\hline WVE15840-4 & 26973|WVE15840 & 7/7/08 9:06 & 302.0578 & 4 \\
\hline WVE15840-5 & \begin{tabular}{l|l|l|}
27207 & WVE15840
\end{tabular} & $7 / 8 / 08$ 14:35 & 302.0747 & 5 \\
\hline WVE15840-6 & \begin{tabular}{l|l|l|}
27272 & WVE15840 \\
\end{tabular} & 7/8/08 20:59 & 302.0667 & 6 \\
\hline WVE15840-7 & 27364|WVE15840 & 7/9/08 9:52 & 302.0636 & 7 \\
\hline WVE15840-8 & 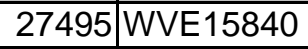 & 7/10/08 15:12 & 302.0556 & 8 \\
\hline WVE15840-9 & \begin{tabular}{l|l}
27602 & WVE15840
\end{tabular} & 7/11/08 9:43 & 302.0538 & 9 \\
\hline WVE15840-10 & \begin{tabular}{l|l|}
27846 & WVE15840 \\
\end{tabular} & $7 / 14 / 08$ 12:22 & 302.0514 & 10 \\
\hline WVE15840-11 & \begin{tabular}{l|l|}
28166 & WVE15840
\end{tabular} & 7/18/08 13:27 & 302.0468 & 11 \\
\hline WVE15840-12 & 28773|WVE15840 & $7 / 25 / 08$ 13:46 & 302.0455 & 12 \\
\hline WVE15840-13 & 29168 WVE15840 & 7/30/08 11:17 & 302.0454 & 13 \\
\hline WVE15840-14 & \begin{tabular}{l|l|}
29566 & WVE15840 \\
\end{tabular} & $8 / 7 / 0817: 31$ & 302.0454 & 14 \\
\hline WVE15840-15 & \begin{tabular}{l|l|}
29922 & WVE15840
\end{tabular} & 8/14/08 16:38 & 302.0472 & 15 \\
\hline WVE15840-16 & \begin{tabular}{l|l|l}
30092 & WVE15840
\end{tabular} & 8/21/08 10:48 & 302.0496 & 16 \\
\hline WVE15840-17 & \begin{tabular}{l|l|}
30347 & WVE15840
\end{tabular} & $8 / 28 / 08$ 10:24 & 302.0532 & 17 \\
\hline WVE15840-18 & \begin{tabular}{l|l|l}
30569 & WVE15840
\end{tabular} & 9/3/08 16:49 & 302.0589 & 18 \\
\hline WVE15841-1 & \begin{tabular}{l|l|l}
26661 & WVE15841 \\
\end{tabular} & 7/2/08 13:44 & 299.104 & 1 \\
\hline WVE15841-2 & 26768|WVE15841 & $7 / 3 / 089: 35$ & 299.0979 & 2 \\
\hline WVE15841-3 & \begin{tabular}{l|l|l|}
26872 & WVE15841 \\
\end{tabular} & 7/3/08 14:18 & 299.0976 & 3 \\
\hline WVE15841-4 & 26974|WVE15841 & 7/7/08 9:06 & 299.0915 & 4 \\
\hline WVE15841-5 & 27208 $\mid$ WVE15841 & 7/8/08 14:36 & 298.8631 & 5 \\
\hline WVE15841-6 & 27271|WVE15841 & 7/8/08 20:58 & 298.8645 & 6 \\
\hline WVE15841-7 & \begin{tabular}{l|l|}
27367 & WVE15841 \\
\end{tabular} & 7/9/08 9:55 & 298.8673 & 7 \\
\hline WVE15841-8 & \begin{tabular}{l|l|}
27498 & WVE15841
\end{tabular} & 7/10/08 15:17 & 298.8723 & 8 \\
\hline WVE15841-9 & $27605 \mid$ WVE15841 & 7/11/08 9:46 & 298.8726 & 9 \\
\hline WVE15841-10 & \begin{tabular}{l|l}
27849 & WVE15841
\end{tabular} & $7 / 14 / 08$ 12:27 & 298.8806 & 10 \\
\hline WVE15841-11 & \begin{tabular}{l|l|}
28168 & WVE15841 \\
\end{tabular} & $7 / 18 / 08$ 13:31 & 298.8881 & 11 \\
\hline WVE15841-12 & \begin{tabular}{l|l}
28785 & WVE15841
\end{tabular} & $7 / 25 / 08$ 15:14 & 298.8972 & 12 \\
\hline WVE15841-13 & 29171 $\mid$ WVE15841 & $7 / 30 / 08$ 11:19 & 298.9068 & 13 \\
\hline WVE15841-14 & \begin{tabular}{l|l|l}
29569 & WVE15841
\end{tabular} & $8 / 7 / 08$ 17:35 & 298.9117 & 14 \\
\hline WVE15842-1 & \begin{tabular}{l|l}
26662 & WVE15842
\end{tabular} & $7 / 2 / 08$ 13:45 & 291.8841 & 1 \\
\hline
\end{tabular}




\begin{tabular}{|c|c|c|c|c|}
\hline WVE15842-2 & 26769|WVE15842 & 7/3/08 9:36 & 291.8824 & 2 \\
\hline WVE15842-3 & \begin{tabular}{l|l|l}
26873 & WVE15842
\end{tabular} & $7 / 3 / 08$ 14:19 & 291.8808 & 3 \\
\hline WVE15842-4 & \begin{tabular}{l|l|l|}
26977 & WVE15842
\end{tabular} & 7/7/08 9:11 & 291.8761 & 4 \\
\hline WVE15842-5 & \begin{tabular}{l|l}
27209 & WVE15842
\end{tabular} & $7 / 8 / 08$ 14:37 & 291.656 & 5 \\
\hline WVE15842-6 & 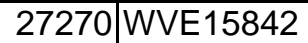 & 7/8/08 20:57 & 291.6564 & 6 \\
\hline WVE15842-7 & 27370|WVE15842 & 7/9/08 9:58 & 291.6623 & 7 \\
\hline WVE15842-8 & \begin{tabular}{|l|l|}
27499 & WVE15842
\end{tabular} & $7 / 10 / 08$ 15:19 & 291.6656 & 8 \\
\hline WVE15842-9 & \begin{tabular}{l|l|l}
27606 & WVE15842 \\
\end{tabular} & 7/11/08 9:47 & 291.6673 & 9 \\
\hline WVE15842-10 & 27850 WVE15842 & $7 / 14 / 08$ 12:28 & 291.6733 & 10 \\
\hline WVE15842-11 & \begin{tabular}{l|l|l|}
28169 & WVE15842 \\
\end{tabular} & 7/18/08 13:32 & 291.6765 & 11 \\
\hline WVE15842-12 & \begin{tabular}{l|l|l|}
28786 & WVE15842
\end{tabular} & $7 / 25 / 08$ 15:15 & 291.6864 & 12 \\
\hline WVE15842-13 & \begin{tabular}{l|l|l}
29172 & WVE15842
\end{tabular} & $7 / 30 / 08$ 11:20 & 291.6939 & 13 \\
\hline WVE15842-14 & 29570|WVE15842 & 8/7/08 17:37 & 291.6973 & 14 \\
\hline WVE15843-1 & \begin{tabular}{l|l|l}
26663 & WVE15843
\end{tabular} & $7 / 2 / 08$ 13:46 & 291.4473 & 1 \\
\hline WVE15843-2 & \begin{tabular}{l|l|l|}
26770 & WVE15843 \\
\end{tabular} & 7/3/08 9:37 & 291.4423 & 2 \\
\hline WVE15843-3 & 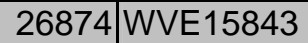 & $7 / 3 / 08$ 14:20 & 291.4407 & 3 \\
\hline WVE15843-4 & 26978|WVE15843 & 7/7/08 9:12 & 291.4376 & 4 \\
\hline WVE15843-5 & \begin{tabular}{|l|l|}
27210 & WVE15843 \\
\end{tabular} & 7/8/08 14:37 & 291.243 & 5 \\
\hline WVE15843-6 & 27269 WVE15843 & 7/8/08 20:56 & 291.2444 & 6 \\
\hline WVE15843-7 & \begin{tabular}{l|l|l|}
27371 & WVE15843 \\
\end{tabular} & 7/9/08 9:59 & 291.2505 & 7 \\
\hline WVE15843-8 & 27500 WVE15843 & $7 / 10 / 08$ 15:20 & 291.2516 & 8 \\
\hline WVE15843-9 & \begin{tabular}{l|l|l|}
27607 & WVE15843 \\
\end{tabular} & 7/11/08 9:48 & 291.2558 & 9 \\
\hline WVE15843-10 & \begin{tabular}{l|l|l|}
27851 & WVE15843 \\
\end{tabular} & $7 / 14 / 08$ 12:29 & 291.2625 & 10 \\
\hline WVE15843-11 & 28170|WVE15843 & 7/18/08 13:34 & 291.2663 & 11 \\
\hline WVE15843-12 & 28787 $\mid$ WVE15843 & $7 / 25 / 08$ 15:17 & 291.2744 & 12 \\
\hline WVE15843-13 & 29173 WVE15843 & 7/30/08 11:21 & 291.2814 & 13 \\
\hline WVE15843-14 & 29571|WVE15843 & 8/7/08 17:38 & 291.288 & 14 \\
\hline WVE15844-1 & \begin{tabular}{l|l|l|}
26664 & WVE15844 \\
\end{tabular} & 7/2/08 13:47 & 292.0032 & 1 \\
\hline WVE15844-2 & \begin{tabular}{l|l|l|}
26771 & WVE15844 \\
\end{tabular} & 7/3/08 9:38 & 291.9991 & 2 \\
\hline WVE15844-3 & \begin{tabular}{l|l|l|}
26875 & WVE15844 \\
\end{tabular} & $7 / 3 / 08$ 14:21 & 291.9968 & 3 \\
\hline WVE15844-4 & 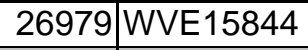 & 7/7/08 9:12 & 291.9939 & 4 \\
\hline WVE15844-5 & \begin{tabular}{|l|l|}
27211 & WVE15844 \\
\end{tabular} & 7/8/08 14:39 & 291.7771 & 5 \\
\hline WVE15844-6 & 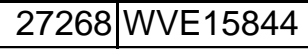 & 7/8/08 20:55 & 291.7797 & 6 \\
\hline WVE15844-7 & \begin{tabular}{l|l|l|}
27372 & WVE15844 \\
\end{tabular} & 7/9/08 9:59 & 291.7834 & 7 \\
\hline WVE15844-8 & \begin{tabular}{l|l|l|}
27501 & WVE15844 \\
\end{tabular} & $7 / 10 / 08$ 15:21 & 291.7855 & 8 \\
\hline WVE15844-9 & \begin{tabular}{l|l|l|}
27608 & WVE15844 \\
\end{tabular} & 7/11/08 9:49 & 291.7899 & 9 \\
\hline WVE15844-10 & \begin{tabular}{l|l|l|}
27852 & WVE15844 \\
\end{tabular} & $7 / 14 / 08$ 12:30 & 291.7971 & 10 \\
\hline WVE15844-11 & \begin{tabular}{l|l|l|}
28171 & WVE15844 \\
\end{tabular} & 7/18/08 13:35 & 291.803 & 11 \\
\hline WVE15844-12 & \begin{tabular}{|l|l|l|}
28788 & WVE15844 \\
\end{tabular} & 7/25/08 15:18 & 291.8094 & 12 \\
\hline WVE15844-13 & \begin{tabular}{l|l|l|}
29174 & WVE15844 \\
\end{tabular} & $7 / 30 / 08$ 11:22 & 291.8168 & 13 \\
\hline WVE15844-14 & \begin{tabular}{l|l|l|} 
29572 & WVE15844
\end{tabular} & 8/7/08 17:38 & 291.8237 & 14 \\
\hline WVE15845-1 & 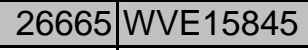 & 7/2/08 13:48 & 299.9256 & 1 \\
\hline WVE15845-2 & \begin{tabular}{l|l|l|}
26772 & WVE15845 \\
\end{tabular} & 7/3/08 9:39 & 299.9214 & 2 \\
\hline WVE15845-3 & $\begin{array}{l}26876 \\
\end{array}$ & $7 / 3 / 08$ 14:21 & 299.9198 & 3 \\
\hline WVE15845-4 & \begin{tabular}{l|l|l|}
26980 & WVE15845 \\
\end{tabular} & 7/7/08 9:13 & 299.9138 & 4 \\
\hline WVE15845-5 & \begin{tabular}{l|l|l}
27212 & WVE15845 \\
\end{tabular} & $7 / 8 / 08$ 14:40 & 299.7087 & 5 \\
\hline WVE15845-6 & \begin{tabular}{l|l|l|}
27267 & WVE15845 \\
\end{tabular} & 7/8/08 20:54 & 299.7079 & 6 \\
\hline WVE15845-7 & 27373 WVE15845 & 7/9/08 10:00 & 299.7133 & 7 \\
\hline WVE15845-8 & \begin{tabular}{l|l|l|}
27502 & WVE15845 \\
\end{tabular} & 7/10/08 15:22 & 299.7148 & 8 \\
\hline
\end{tabular}




\begin{tabular}{|c|c|c|c|c|}
\hline WVE15845-9 & \begin{tabular}{l|l|l|}
27611 & WVE15845 \\
\end{tabular} & 7/11/08 9:52 & 299.7196 & 9 \\
\hline WVE15845-10 & \begin{tabular}{l|l|l|}
27853 & WVE15845
\end{tabular} & $7 / 14 / 08$ 12:31 & 299.7297 & 10 \\
\hline WVE15845-11 & \begin{tabular}{|l|l|}
28172 & WVE15845 \\
\end{tabular} & $7 / 18 / 08$ 13:36 & 299.7297 & 11 \\
\hline WVE15845-12 & \begin{tabular}{l|l|}
28789 & WVE15845 \\
\end{tabular} & $7 / 25 / 08$ 15:19 & 299.7369 & 12 \\
\hline WVE15845-13 & \begin{tabular}{l|l|l|}
29175 & WVE15845
\end{tabular} & 7/30/08 11:22 & 299.7501 & 13 \\
\hline WVE15845-14 & 29573|WVE15845 & $8 / 7 / 0817: 40$ & 299.7573 & 14 \\
\hline WVE15846-1 & \begin{tabular}{l|l|l}
26666 & WVE15846
\end{tabular} & $7 / 2 / 08$ 13:49 & 304.36 & 1 \\
\hline WVE15846-2 & \begin{tabular}{l|l|l|}
26773 & WVE15846
\end{tabular} & $7 / 3 / 089: 40$ & 304.355 & 2 \\
\hline WVE15846-3 & \begin{tabular}{l|l|l|}
26877 & WVE15846 \\
\end{tabular} & $7 / 3 / 08$ 14:22 & 304.3542 & 3 \\
\hline WVE15846-4 & \begin{tabular}{l|l|}
26981 & WVE15846
\end{tabular} & 7/7/08 9:14 & 304.3498 & 4 \\
\hline WVE15846-5 & \begin{tabular}{l|l|l|}
27215 & WVE15846 \\
\end{tabular} & 7/8/08 14:47 & 303.4594 & 5 \\
\hline WVE15846-6 & 27284|WVE15846 & $7 / 8 / 08$ 21:16 & 303.4606 & 6 \\
\hline WVE15846-7 & \begin{tabular}{l|l|l|}
27374 & WVE15846
\end{tabular} & 7/9/08 10:01 & 303.4572 & 7 \\
\hline WVE15846-8 & \begin{tabular}{|l|l|}
27503 & WVE15846
\end{tabular} & 7/10/08 15:23 & 303.4544 & 8 \\
\hline WVE15846-9 & \begin{tabular}{l|l}
27612 & WVE15846 \\
\end{tabular} & 7/11/08 9:52 & 303.4537 & 9 \\
\hline WVE15846-10 & 27854|WVE15846 & $7 / 14 / 08$ 12:32 & 303.4594 & 10 \\
\hline WVE15846-11 & \begin{tabular}{l|l|l}
28173 & WVE15846
\end{tabular} & 7/18/08 13:37 & 303.4593 & 11 \\
\hline WVE15846-12 & \begin{tabular}{l|l|l|}
28790 & WVE15846
\end{tabular} & $7 / 25 / 08$ 15:20 & 303.4647 & 12 \\
\hline WVE15846-13 & \begin{tabular}{l|l|l}
29176 & WVE15846 \\
\end{tabular} & $7 / 30 / 08$ 11:23 & 303.4718 & 13 \\
\hline WVE15846-14 & 29574|WVE15846 & $8 / 7 / 0817: 40$ & 303.4776 & 14 \\
\hline WVE15847-1 & \begin{tabular}{l|l|}
26667 & WVE15847
\end{tabular} & $7 / 2 / 08$ 13:50 & 308.1919 & 1 \\
\hline WVE15847-2 & \begin{tabular}{l|l|l|}
26774 & WVE15847 \\
\end{tabular} & 7/3/08 9:41 & 308.1874 & 2 \\
\hline WVE15847-3 & \begin{tabular}{l|l}
26878 & WVE15847
\end{tabular} & $7 / 3 / 08$ 14:23 & 308.1858 & 3 \\
\hline WVE15847-4 & \begin{tabular}{l|l|l}
26982 & WVE15847 \\
\end{tabular} & 7/7/08 9:15 & 308.1796 & 4 \\
\hline WVE15847-5 & \begin{tabular}{l|l|} 
27216 & WVE15847
\end{tabular} & 7/8/08 14:48 & 307.63 & 5 \\
\hline WVE15847-6 & \begin{tabular}{l|l|l|}
27285 & WVE15847 \\
\end{tabular} & $7 / 8 / 0821: 17$ & 307.6316 & 6 \\
\hline WVE15847-7 & 27375|WVE15847 & 7/9/08 10:02 & 307.6301 & 7 \\
\hline WVE15847-8 & \begin{tabular}{l|l|l|}
27504 & WVE15847
\end{tabular} & $7 / 10 / 08$ 15:24 & 307.6224 & 8 \\
\hline WVE15847-9 & \begin{tabular}{|l|l|}
27613 & $W V E 15847$
\end{tabular} & 7/11/08 9:53 & 307.6214 & 9 \\
\hline WVE15847-10 & \begin{tabular}{l|l|l|}
27855 & WVE15847 \\
\end{tabular} & $7 / 14 / 08$ 12:32 & 307.6234 & 10 \\
\hline WVE15847-11 & \begin{tabular}{l|l|}
28174 & WVE15847 \\
\end{tabular} & 7/18/08 13:38 & 307.6223 & 11 \\
\hline WVE15847-12 & \begin{tabular}{l|l}
28791 & WVE15847
\end{tabular} & $7 / 25 / 08$ 15:21 & 307.6295 & 12 \\
\hline WVE15847-13 & \begin{tabular}{l|l|}
29177 & WVE15847
\end{tabular} & $7 / 30 / 08$ 11:24 & 307.6348 & 13 \\
\hline WVE15847-14 & \begin{tabular}{l|l|l}
29575 & WVE15847
\end{tabular} & $8 / 7 / 0817: 41$ & 307.6376 & 14 \\
\hline WVE15848-1 & \begin{tabular}{l|l}
26668 & WVE15848
\end{tabular} & 7/2/08 13:51 & 307.4319 & 1 \\
\hline WVE15848-2 & $26775 \mid$ WVE15848 & $7 / 3 / 089: 41$ & 307.4278 & 2 \\
\hline WVE15848-3 & \begin{tabular}{l|l|l|}
26879 & WVE15848 \\
\end{tabular} & $7 / 3 / 0814: 24$ & 307.426 & 3 \\
\hline WVE15848-4 & 26983|WVE15848 & 7/7/08 9:16 & 307.422 & 4 \\
\hline WVE15848-5 & \begin{tabular}{l|l|l|}
27217 & WVE15848 \\
\end{tabular} & $7 / 8 / 08$ 14:49 & 306.8073 & 5 \\
\hline WVE15848-6 & \begin{tabular}{l|l|l}
27286 & WVE15848
\end{tabular} & $7 / 8 / 08$ 21:18 & 306.8079 & 6 \\
\hline WVE15848-7 & \begin{tabular}{l|l|}
27376 & WVE15848 \\
\end{tabular} & 7/9/08 10:03 & 306.8054 & 7 \\
\hline WVE15848-8 & \begin{tabular}{l|l|l|}
27505 & WVE15848
\end{tabular} & $7 / 10 / 08$ 15:25 & 306.8013 & 8 \\
\hline WVE15848-9 & \begin{tabular}{l|l|l|}
27614 & WVE15848 \\
\end{tabular} & 7/11/08 9:54 & 306.8 & 9 \\
\hline WVE15848-10 & \begin{tabular}{l|l}
27856 & WVE15848
\end{tabular} & $7 / 14 / 08$ 12:33 & 306.803 & 10 \\
\hline WVE15848-11 & \begin{tabular}{l|l|}
28175 & WVE15848 \\
\end{tabular} & $7 / 18 / 08$ 13:38 & 306.7999 & 11 \\
\hline WVE15848-12 & \begin{tabular}{l|l|l|} 
28792 & WVE15848
\end{tabular} & $7 / 25 / 08$ 15:22 & 306.8071 & 12 \\
\hline WVE15848-13 & \begin{tabular}{l|l|}
29178 & WVE15848
\end{tabular} & $7 / 30 / 08$ 11:24 & 306.8123 & 13 \\
\hline WVE15848-14 & \begin{tabular}{l|l|l}
29576 & WVE15848 \\
\end{tabular} & 8/7/08 17:42 & 306.8167 & 14 \\
\hline WVE15849-1 & \begin{tabular}{l|l}
26669 & WVE15849
\end{tabular} & $7 / 2 / 08$ 13:51 & 306.6688 & 1 \\
\hline
\end{tabular}




\begin{tabular}{|c|c|c|c|c|}
\hline WVE15849-2 & \begin{tabular}{|l|l|}
26776 & WVE15849
\end{tabular} & 7/3/08 9:42 & 306.6572 & 2 \\
\hline WVE15849-3 & \begin{tabular}{l|l|l|}
26880 & WVE15849
\end{tabular} & $7 / 3 / 0814: 24$ & 306.6509 & 3 \\
\hline WVE15849-4 & 26984 $\mid$ WVE15849 & 7/7/08 9:17 & 306.648 & 4 \\
\hline WVE15849-5 & \begin{tabular}{l|l}
27218 & WVE15849
\end{tabular} & $7 / 8 / 08$ 14:49 & 305.9353 & 5 \\
\hline WVE15849-6 & \begin{tabular}{|l|l|}
27287 & WVE15849
\end{tabular} & 7/8/08 21:19 & 305.9341 & 6 \\
\hline WVE15849-7 & 27377|WVE15849 & 7/9/08 10:03 & 305.9353 & 7 \\
\hline WVE15849-8 & \begin{tabular}{|l|l|}
27506 & WVE15849
\end{tabular} & $7 / 10 / 08$ 15:25 & 305.93 & 8 \\
\hline WVE15849-9 & \begin{tabular}{l|l|l|}
27615 & WVE15849 \\
\end{tabular} & 7/11/08 9:54 & 305.9274 & 9 \\
\hline WVE15849-10 & \begin{tabular}{l|l|l|}
27857 & WVE15849 \\
\end{tabular} & $7 / 14 / 08$ 12:34 & 305.9296 & 10 \\
\hline WVE15849-11 & \begin{tabular}{l|l|l|}
28176 & WVE15849 \\
\end{tabular} & 7/18/08 13:39 & 305.9277 & 11 \\
\hline WVE15849-12 & 28793|WVE15849 & $7 / 25 / 08$ 15:23 & 305.9345 & 12 \\
\hline WVE15849-13 & 29179 WVE15849 & $7 / 30 / 08$ 11:25 & 305.941 & 13 \\
\hline WVE15849-14 & \begin{tabular}{l|l|l|}
29577 & WVE15849
\end{tabular} & 8/7/08 17:43 & 305.9433 & 14 \\
\hline WVE15850-1 & \begin{tabular}{l|l|}
26670 & WVE15850
\end{tabular} & $7 / 2 / 08$ 13:52 & 307.4325 & 1 \\
\hline WVE15850-2 & \begin{tabular}{l|l|l|}
26777 & WVE15850 \\
\end{tabular} & 7/3/08 9:43 & 307.4281 & 2 \\
\hline WVE15850-3 & \begin{tabular}{|l|l|}
268815850 & WVE15 \\
\end{tabular} & $7 / 3 / 08$ 14:25 & 307.4272 & 3 \\
\hline WVE15850-4 & $26985 \mid$ WVE15850 & 7/7/08 9:18 & 307.4242 & 4 \\
\hline WVE15850-5 & 27219 WVE15850 & $7 / 8 / 08$ 14:50 & 306.6602 & 5 \\
\hline WVE15850-6 & \begin{tabular}{|l|l|}
27288 & WVE15850 \\
\end{tabular} & $7 / 8 / 0821: 20$ & 306.656 & 6 \\
\hline WVE15850-7 & \begin{tabular}{l|l|l}
27378 & WVE15850 \\
\end{tabular} & 7/9/08 10:04 & 306.6541 & 7 \\
\hline WVE15850-8 & \begin{tabular}{l|l|l|}
27507 & WVE15850 \\
\end{tabular} & $7 / 10 / 08$ 15:26 & 306.6494 & 8 \\
\hline WVE15850-9 & \begin{tabular}{l|l|l}
27616 & WVE15850 \\
\end{tabular} & 7/11/08 9:55 & 306.6476 & 9 \\
\hline WVE15850-10 & 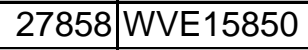 & $7 / 14 / 08$ 12:35 & 306.6516 & 10 \\
\hline WVE15850-11 & 28177|WVE15850 & 7/18/08 13:40 & 306.6457 & 11 \\
\hline WVE15850-12 & 28794|WVE15850 & $7 / 25 / 08$ 15:24 & 306.6584 & 12 \\
\hline WVE15850-13 & \begin{tabular}{l|l|}
29180 & WVE15850 \\
\end{tabular} & $7 / 30 / 08$ 11:26 & 306.6626 & 13 \\
\hline WVE15850-14 & 29578|WVE15850 & $8 / 7 / 0817: 44$ & 306.6682 & 14 \\
\hline WVE15851-1 & 26673 WVE15851 & 7/2/08 13:55 & 305.9663 & 1 \\
\hline WVE15851-2 & \begin{tabular}{l|l|l}
26780 & WVE15851 \\
\end{tabular} & 7/3/08 9:47 & 305.9606 & 2 \\
\hline WVE15851-3 & \begin{tabular}{l|l|}
26884 & WVE15851
\end{tabular} & $7 / 3 / 08$ 14:29 & 305.9598 & 3 \\
\hline WVE15851-4 & \begin{tabular}{|l|l|}
26986 & WVE15851 \\
\end{tabular} & 7/7/08 9:19 & 305.9563 & 4 \\
\hline WVE15851-5 & \begin{tabular}{l|l|l}
27070 & WVE15851 \\
\end{tabular} & $7 / 7 / 08$ 17:45 & 306.7565 & 5 \\
\hline WVE15851-6 & 27139|WVE15851 & 7/8/08 9:38 & 306.7578 & 6 \\
\hline WVE15851-7 & \begin{tabular}{l|l|l}
27381 & WVE15851 \\
\end{tabular} & 7/9/08 10:08 & 306.7583 & 7 \\
\hline WVE15851-8 & \begin{tabular}{l|l|l}
27510 & WVE15851 \\
\end{tabular} & 7/10/08 15:31 & 306.7593 & 8 \\
\hline WVE15851-9 & 27619 WVE15851 & 7/11/08 9:59 & 306.7588 & 9 \\
\hline WVE15851-10 & \begin{tabular}{l|l|l|}
27861 & WVE15851 \\
\end{tabular} & $7 / 14 / 08$ 12:40 & 306.7617 & 10 \\
\hline WVE15851-11 & \begin{tabular}{l|l|l}
28180 & WVE15851 \\
\end{tabular} & 7/18/08 13:44 & 306.7562 & 11 \\
\hline WVE15851-12 & \begin{tabular}{l|l|l|}
28797 & WVE15851 \\
\end{tabular} & $7 / 25 / 08$ 15:30 & 306.7603 & 12 \\
\hline WVE15851-13 & \begin{tabular}{l|l|l|}
29183 & WVE15851 \\
\end{tabular} & 7/30/08 11:28 & 306.7629 & 13 \\
\hline WVE15851-14 & \begin{tabular}{l|l|}
29581 & WVE15851 \\
\end{tabular} & 8/7/08 17:47 & 306.7632 & 14 \\
\hline WVE15852-1 & 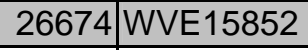 & 7/2/08 13:56 & 303.6411 & 1 \\
\hline WVE15852-2 & \begin{tabular}{l|l|l}
26781 & WVE15852 \\
\end{tabular} & 7/3/08 9:47 & 303.637 & 2 \\
\hline WVE15852-3 & 26885 WVE15852 & $7 / 3 / 08$ 14:29 & 303.6344 & 3 \\
\hline WVE15852-4 & \begin{tabular}{l|l|l}
26990 & WVE15852 \\
\end{tabular} & 7/7/08 9:23 & 303.6294 & 4 \\
\hline WVE15852-5 & 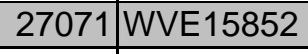 & $7 / 7 / 08$ 17:46 & 304.5129 & 5 \\
\hline WVE15852-6 & \begin{tabular}{l|l|l|}
27140 & WVE15852 \\
\end{tabular} & 7/8/08 9:39 & 304.5098 & 6 \\
\hline WVE15852-7 & \begin{tabular}{l|l|l|}
27382 & WVE15852 \\
\end{tabular} & 7/9/08 10:09 & 304.5111 & 7 \\
\hline WVE15852-8 & \begin{tabular}{l|l|l|}
27511 & WVE15852 \\
\end{tabular} & 7/10/08 15:32 & 304.5094 & 8 \\
\hline
\end{tabular}




\begin{tabular}{|c|c|c|c|c|}
\hline WVE15852-9 & \begin{tabular}{l|l|l} 
27620 & WVE15852 \\
\end{tabular} & 7/11/08 10:00 & 304.5085 & 9 \\
\hline WVE15852-10 & \begin{tabular}{l|l|l|}
27862 & WVE15852
\end{tabular} & 7/14/08 12:41 & 304.5078 & 10 \\
\hline WVE15852-11 & 28181 WVE15852 & $7 / 18 / 08$ 13:45 & 304.5042 & 11 \\
\hline WVE15852-12 & 28798 $\mid$ WVE15852 & 7/25/08 15:31 & 304.5042 & 12 \\
\hline WVE15852-13 & 29184 $\mid$ WVE15852 & $7 / 30 / 08$ 11:29 & 304.5055 & 13 \\
\hline WVE15852-14 & 29582|WVE15852 & 8/7/08 17:48 & 304.5067 & 14 \\
\hline WVE15853-1 & $26675 \mid$ WVE15853 & $7 / 2 / 08$ 13:57 & 307.8383 & 1 \\
\hline WVE15853-2 & \begin{tabular}{l|l|}
26782 & WVE15853
\end{tabular} & 7/3/08 9:48 & 307.8348 & 2 \\
\hline WVE15853-3 & \begin{tabular}{|l|l|} 
26886 & WVE15853 \\
\end{tabular} & $7 / 3 / 08$ 14:30 & 307.8322 & 3 \\
\hline WVE15853-4 & 26991|WVE15853 & $7 / 7 / 089: 24$ & 307.8278 & 4 \\
\hline WVE15853-5 & \begin{tabular}{|l|l|}
27072 & WVE15853 \\
\end{tabular} & 7/7/08 17:47 & 308.2858 & 5 \\
\hline WVE15853-6 & \begin{tabular}{l|l}
27141 & WVE15853 \\
\end{tabular} & 7/8/08 9:40 & 308.2829 & 6 \\
\hline WVE15853-7 & 27383 WVE15853 & 7/9/08 10:09 & 308.2847 & 7 \\
\hline WVE15853-8 & 27512 $\mid$ WVE15853 & $7 / 10 / 08$ 15:32 & 308.2832 & 8 \\
\hline WVE15853-9 & 27621|WVE15853 & 7/11/08 10:01 & 308.2831 & 9 \\
\hline WVE15853-10 & $27863 \mid$ WVE15853 & $7 / 14 / 08$ 12:42 & 308.2832 & 10 \\
\hline WVE15853-11 & \begin{tabular}{l|l|l|}
28182 & WVE15853 \\
\end{tabular} & $7 / 18 / 08$ 13:46 & 308.2804 & 11 \\
\hline WVE15853-12 & $28799 \mid$ WVE15853 & $7 / 25 / 08$ 15:32 & 308.2821 & 12 \\
\hline WVE15853-13 & \begin{tabular}{|l|l|}
29185 & WVE15853 \\
\end{tabular} & 7/30/08 11:29 & 308.2843 & 13 \\
\hline WVE15853-14 & 29583|WVE15853 & 8/7/08 17:49 & 308.2874 & 14 \\
\hline WVE15854-1 & \begin{tabular}{l|l|}
26676 & WVE15854 \\
\end{tabular} & $7 / 2 / 08$ 13:58 & 309.5539 & 1 \\
\hline WVE15854-2 & \begin{tabular}{|l|l|}
26783 & WVE15854 \\
\end{tabular} & 7/3/08 9:49 & 309.5477 & 2 \\
\hline WVE15854-3 & \begin{tabular}{l|l|}
26887 & WVE15854 \\
\end{tabular} & $7 / 3 / 08$ 14:31 & 309.546 & 3 \\
\hline WVE15854-4 & \begin{tabular}{|l|l|} 
26992 & WVE15854 \\
\end{tabular} & 7/7/08 9:24 & 309.5423 & 4 \\
\hline WVE15854-5 & \begin{tabular}{l|l}
27073 & WVE15854
\end{tabular} & 7/7/08 17:47 & 310.4699 & 5 \\
\hline WVE15854-6 & \begin{tabular}{l|l|}
27142 & WVE15854
\end{tabular} & 7/8/08 9:41 & 310.4651 & 6 \\
\hline WVE15854-7 & \begin{tabular}{l|l|l}
27384 & WVE15854
\end{tabular} & 7/9/08 10:10 & 310.4674 & 7 \\
\hline WVE15854-8 & \begin{tabular}{l|l|}
27513 & WVE15854
\end{tabular} & 7/10/08 15:33 & 310.4656 & 8 \\
\hline WVE15854-9 & \begin{tabular}{l|l|}
27622 & WVE15854 \\
\end{tabular} & 7/11/08 10:02 & 310.4646 & 9 \\
\hline WVE15854-10 & \begin{tabular}{l|l|}
27864 & WVE15854
\end{tabular} & 7/14/08 12:43 & 310.4654 & 10 \\
\hline WVE15854-11 & \begin{tabular}{|l|l|}
28183 & WVE15854 \\
\end{tabular} & 7/18/08 13:47| & 310.462 & 11 \\
\hline WVE15854-12 & \begin{tabular}{l|l|l}
28800 & WVE15854
\end{tabular} & $7 / 25 / 08$ 15:32 & 310.4631 & 12 \\
\hline WVE15854-13 & \begin{tabular}{|l|l|} 
29186 & WVE15854 \\
\end{tabular} & 7/30/08 11:30 & 310.466 & 13 \\
\hline WVE15854-14 & \begin{tabular}{l|l|l}
29584 & WVE15854
\end{tabular} & $8 / 7 / 08$ 17:50 & 310.467 & 14 \\
\hline WVE15855-1 & \begin{tabular}{l|l|l|}
26677 & WVE15855 \\
\end{tabular} & $7 / 2 / 08$ 13:59 & 305.5694 & 1 \\
\hline WVE15855-2 & 26784|WVE15855 & $7 / 3 / 089: 50$ & 305.5693 & 2 \\
\hline WVE15855-3 & \begin{tabular}{|l|l|}
26888 & WVE15855 \\
\end{tabular} & $7 / 3 / 08$ 14:32 & 305.568 & 3 \\
\hline WVE15855-4 & 26993|WVE15855 & 7/7/08 9:25 & 305.5622 & 4 \\
\hline WVE15855-5 & $27074 \mid$ WVE15855 & 7/7/08 17:48 & 305.7833 & 5 \\
\hline WVE15855-6 & \begin{tabular}{l|l|}
27143 & WVE15855 \\
\end{tabular} & 7/8/08 9:42 & 305.78 & 6 \\
\hline WVE15855-7 & 27385|WVE15855 & 7/9/08 10:11 & 305.7836 & 7 \\
\hline WVE15855-8 & \begin{tabular}{l|l|}
27514 & WVE15855 \\
\end{tabular} & 7/10/08 15:34 & 305.7777 & 8 \\
\hline WVE15855-9 & 27623 $\mid$ WVE15855 & 7/11/08 10:02 & 305.7793 & 9 \\
\hline WVE15855-10 & \begin{tabular}{l|l|l|}
27865 & WVE15855
\end{tabular} & $7 / 14 / 08$ 12:44 & 305.7804 & 10 \\
\hline WVE15855-11 & 28184 $\mid$ WVE15855 & 7/18/08 13:48 & 305.7812 & 11 \\
\hline WVE15855-12 & $28801 \mid$ WVE15855 & $7 / 25 / 08$ 15:34 & 305.7841 & 12 \\
\hline WVE15855-13 & \begin{tabular}{|l|l|}
29187 & WVE15855 \\
\end{tabular} & 7/30/08 11:31 & 305.7862 & 13 \\
\hline WVE15855-14 & 29585|WVE15855 & 8/7/08 17:51 & 305.7927 & 14 \\
\hline WVE15856-1 & \begin{tabular}{l|l|}
26678 & WVE15856
\end{tabular} & 7/2/08 13:59 & 299.1227 & 1 \\
\hline
\end{tabular}




\begin{tabular}{|c|c|c|c|c|}
\hline WVE15856-2 & $26785 \mid$ WVE15856 & 7/3/08 9:51 & 299.1205 & 2 \\
\hline WVE15856-3 & 26889|WVE15856 & 7/3/08 14:32 & 299.1192 & 3 \\
\hline WVE15856-4 & 26994|WVE15856 & 7/7/08 9:26 & 299.1139 & 4 \\
\hline WVE15856-5 & \begin{tabular}{l|l|l|}
27077 & WVE15856 \\
\end{tabular} & 7/7/08 17:49 & 299.2306 & 5 \\
\hline WVE15856-6 & $\begin{array}{l}27146 \\
\end{array}$ & 7/8/08 9:45 & 299.2335 & 6 \\
\hline WVE15856-7 & \begin{tabular}{|l|l|}
27386 & WVE15856 \\
\end{tabular} & $7 / 9 / 08$ 10:12 & 299.2347 & 7 \\
\hline WVE15856-8 & $27515 \mid$ WVE15856 & 7/10/08 15:35 & 299.234 & 8 \\
\hline WVE15856-9 & \begin{tabular}{l|l|l|}
27624 & WVE15856 \\
\end{tabular} & 7/11/08 10:03 & 299.234 & 9 \\
\hline WVE15856-10 & $\begin{array}{l}27866 \\
\text { WVE15856 }\end{array}$ & $7 / 14 / 08$ 12:45 & 299.2362 & 10 \\
\hline WVE15856-11 & \begin{tabular}{l|l|l|}
28185 & WVE15856 \\
\end{tabular} & 7/18/08 13:49 & 299.2318 & 11 \\
\hline WVE15856-12 & \begin{tabular}{l|l|l|}
28802 & WVE15856 \\
\end{tabular} & $7 / 25 / 08$ 15:34 & 299.2372 & 12 \\
\hline WVE15856-13 & 29188|WVE15856 & 7/30/08 11:32 & 299.2398 & 13 \\
\hline WVE15856-14 & \begin{tabular}{|l|l|}
29586 & WVE15856 \\
\end{tabular} & 8/7/08 17:52 & 299.2406 & 14 \\
\hline WVE15857-1 & 26679 WVE15857 & $7 / 2 / 08$ 14:00 & 295.3658 & 1 \\
\hline WVE15857-2 & \begin{tabular}{l|l|l|}
26786 & WVE15857 \\
\end{tabular} & 7/3/08 9:52 & 295.3627 & 2 \\
\hline WVE15857-3 & \begin{tabular}{|l|l|}
26890 & WVE15857 \\
\end{tabular} & 7/3/08 14:33 & 295.3617 & 3 \\
\hline WVE15857-4 & $26995 \mid$ WVE15857 & 7/7/08 9:27 & 295.3582 & 4 \\
\hline WVE15857-5 & \begin{tabular}{l|l|l}
27078 & WVE15857 \\
\end{tabular} & 7/7/08 17:50 & 295.8547 & 5 \\
\hline WVE15857-6 & \begin{tabular}{l|l|l|}
27147 & WVE15857 \\
\end{tabular} & 7/8/08 9:46 & 295.8575 & 6 \\
\hline WVE15857-7 & \begin{tabular}{l|l|l}
27387 & WVE15857 \\
\end{tabular} & 7/9/08 10:12 & 295.8577 & 7 \\
\hline WVE15857-8 & $\begin{array}{l}27516 \\
\text { WVE15857 }\end{array}$ & 7/10/08 15:36 & 295.8553 & 8 \\
\hline WVE15857-9 & \begin{tabular}{l|l|l}
27625 & WVE15857 \\
\end{tabular} & 7/11/08 10:04 & 295.8553 & 9 \\
\hline WVE15857-10 & \begin{tabular}{l|l|l|}
27867 & WVE15857 \\
\end{tabular} & $7 / 14 / 08$ 12:46 & 295.8559 & 10 \\
\hline WVE15857-11 & \begin{tabular}{l|l|l}
28186 & WVE15857 \\
\end{tabular} & 7/18/08 13:50 & 295.8528 & 11 \\
\hline WVE15857-12 & 28803 WVE15857 & $7 / 25 / 08$ 15:35 & 295.856 & 12 \\
\hline WVE15857-13 & 29189 WVE15857 & 7/30/08 11:32 & 295.859 & 13 \\
\hline WVE15857-14 & \begin{tabular}{l|l|l|}
29587 & WVE15857 \\
\end{tabular} & 8/7/08 17:53 & 295.8619 & 14 \\
\hline WVE15858-1 & \begin{tabular}{|l|l|}
26680 & WVE15858 \\
\end{tabular} & 7/2/08 14:01 & 297.7641 & 1 \\
\hline WVE15858-2 & \begin{tabular}{l|l|l|}
26787 & WVE15858 \\
\end{tabular} & $7 / 3 / 08$ 9:52 & 297.7629 & 2 \\
\hline WVE15858-3 & \begin{tabular}{|l|l|}
26891 & WVE15858 \\
\end{tabular} & 7/3/08 14:34 & 297.7603 & 3 \\
\hline WVE15858-4 & \begin{tabular}{|l|l|}
26996 & WVE15858 \\
\end{tabular} & 7/7/08 9:27 & 297.756 & 4 \\
\hline WVE15858-5 & 27079 WVE15858 & 7/7/08 17:51 & 297.9078 & 5 \\
\hline WVE15858-6 & 27148 WVE15858 & 7/8/08 9:47 & 297.9111 & 6 \\
\hline WVE15858-7 & \begin{tabular}{|l|l|}
27388 & WVE15858 \\
\end{tabular} & 7/9/08 10:13 & 297.9105 & 7 \\
\hline WVE15858-8 & 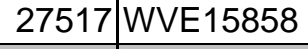 & 7/10/08 15:37 & 297.909 & 8 \\
\hline WVE15858-9 & \begin{tabular}{l|l|l|}
27626 & WVE15858 \\
\end{tabular} & 7/11/08 10:04 & 297.9088 & 9 \\
\hline WVE15858-10 & 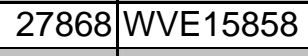 & 7/14/08 12:47 & 297.9096 & 10 \\
\hline WVE15858-11 & \begin{tabular}{|l|l|}
28187 & WVE15858 \\
\end{tabular} & 7/18/08 13:51 & 297.9073 & 11 \\
\hline WVE15858-12 & $28804 \mid$ WVE15858 & 7/25/08 15:36 & 297.9089 & 12 \\
\hline WVE15858-13 & 29190 WVE15858 & 7/30/08 11:33 & 297.9117 & 13 \\
\hline WVE15858-14 & \begin{tabular}{|l|l|}
29588 & WVE15858 \\
\end{tabular} & $8 / 7 / 08$ 17:54 & 297.9149 & 14 \\
\hline WVE15859-1 & \begin{tabular}{l|l|l|}
26681 & WVE15859 \\
\end{tabular} & 7/2/08 14:02 & 306.5764 & 1 \\
\hline WVE15859-2 & 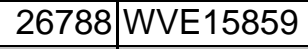 & 7/3/08 9:53 & 306.5765 & 2 \\
\hline WVE15859-3 & 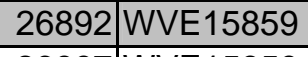 & $7 / 3 / 0814: 34$ & 306.5724 & 3 \\
\hline WVE15859-4 & \begin{tabular}{l|l|l|}
26997 & WVE15859 \\
\end{tabular} & 7/7/08 9:28 & 306.5708 & 4 \\
\hline WVE15859-5 & 27080 WVE15859 & 7/7/08 17:52 & 306.7431 & 5 \\
\hline WVE15859-6 & \begin{tabular}{l|l|l|}
27149 & WVE15859 \\
\end{tabular} & 7/8/08 9:48 & 306.7497 & 6 \\
\hline WVE15859-7 & 27389 WVE15859 & 7/9/08 10:14 & 306.7504 & 7 \\
\hline WVE15859-8 & 27518|WVE15859 & 7/10/08 15:38 & 306.7471 & 8 \\
\hline
\end{tabular}




\begin{tabular}{|c|c|c|c|c|}
\hline WVE15859-9 & 27627 $\mid$ WVE15859 & 7/11/08 10:05 & 306.7475 & 9 \\
\hline WVE15859-10 & 27869 WVE15859 & $7 / 14 / 08$ 12:48 & 306.7499 & 10 \\
\hline WVE15859-11 & \begin{tabular}{l|l|}
28188 & WVE15859
\end{tabular} & 7/18/08 13:52 & 306.7472 & 11 \\
\hline WVE15859-12 & \begin{tabular}{l|l}
28805 & WVE15859
\end{tabular} & 7/25/08 15:37 & 306.7485 & 12 \\
\hline WVE15859-13 & 29191 $\mid$ WVE15859 & $7 / 30 / 08$ 11:34 & 306.7511 & 13 \\
\hline WVE15859-14 & 29589|WVE15859 & $8 / 7 / 08$ 17:55 & 306.7546 & 14 \\
\hline WVE15860-1 & \begin{tabular}{l|l|}
26682 & WVE15860
\end{tabular} & 7/2/08 14:03 & 304.5032 & 1 \\
\hline WVE15860-2 & \begin{tabular}{l|l|}
26789 & WVE15860
\end{tabular} & 7/3/08 9:54 & 304.5 & 2 \\
\hline WVE15860-3 & 26893|WVE15860 & 7/3/08 14:35 & 304.4988 & 3 \\
\hline WVE15860-4 & 26998|WVE15860 & 7/7/08 9:29 & 304.4942 & 4 \\
\hline WVE15860-5 & \begin{tabular}{l|l|l|}
27081 & WVE15860 \\
\end{tabular} & 7/7/08 17:52 & 304.6185 & 5 \\
\hline WVE15860-6 & $27150 \mid$ WVE15860 & 7/8/08 9:48 & 304.6225 & 6 \\
\hline WVE15860-7 & 27390 WVE15860 & $7 / 9 / 08$ 10:14 & 304.6236 & 7 \\
\hline WVE15860-8 & 27519 WVE15860 & 7/10/08 15:39 & 304.621 & 8 \\
\hline WVE15860-9 & 27628|WVE15860 & 7/11/08 10:06 & 304.6219 & 9 \\
\hline WVE15860-10 & 27870 WVE15860 & $7 / 14 / 08$ 12:49 & 304.6226 & 10 \\
\hline WVE15860-11 & \begin{tabular}{l|l|l}
28189 & WVE15860 \\
\end{tabular} & 7/18/08 13:53 & 304.6174 & 11 \\
\hline WVE15860-12 & \begin{tabular}{l|l|}
28806 & WVE15860
\end{tabular} & $7 / 25 / 08$ 15:38 & 304.6207 & 12 \\
\hline WVE15860-13 & \begin{tabular}{l|l}
29192 & WVE15860 \\
\end{tabular} & $7 / 30 / 08$ 11:35 & 304.6236 & 13 \\
\hline WVE15860-14 & 29590|WVE15860 & $8 / 7 / 08$ 17:56 & 304.627 & 14 \\
\hline WVE15861-1 & 26685 $\mid$ WVE15861 & $7 / 2 / 08$ 14:06 & 305.3732 & 1 \\
\hline WVE15861-2 & \begin{tabular}{l|l|}
26792 & WVE15861 \\
\end{tabular} & 7/3/08 9:58 & 305.3684 & 2 \\
\hline WVE15861-3 & \begin{tabular}{l|l|}
26896 & WVE15861 \\
\end{tabular} & $7 / 3 / 08$ 14:39 & 305.3676 & 3 \\
\hline WVE15861-4 & 26999 WVE15861 & 7/7/08 9:30 & 305.3626 & 4 \\
\hline WVE15861-5 & \begin{tabular}{l|l}
27121 & WVE15861 \\
\end{tabular} & 7/7/08 19:57 & 305.0224 & 5 \\
\hline WVE15861-6 & \begin{tabular}{l|l}
27151 & WVE15861
\end{tabular} & $7 / 8 / 08$ 9:49 & 305.0584 & 6 \\
\hline WVE15861-7 & \begin{tabular}{l|l}
27279 & WVE15861
\end{tabular} & $7 / 8 / 0821: 10$ & 305.0681 & 7 \\
\hline WVE15861-8 & 27393 $\mid$ WVE15861 & $7 / 9 / 08$ 10:18 & 305.0778 & 8 \\
\hline WVE15861-9 & \begin{tabular}{l|l}
27527 & WVE15861
\end{tabular} & 7/10/08 15:47 & 305.0915 & 9 \\
\hline WVE15861-10 & 27631|WVE15861 & 7/11/08 10:09 & 305.0962 & 10 \\
\hline WVE15861-11 & \begin{tabular}{l|l}
27873 & WVE15861
\end{tabular} & 7/14/08 13:08 & 305.1187 & 11 \\
\hline WVE15861-12 & \begin{tabular}{l|l|}
28192 & WVE15861
\end{tabular} & 7/18/08 13:58 & 305.1294 & 12 \\
\hline WVE15861-13 & \begin{tabular}{l|l|l}
28809 & WVE15861
\end{tabular} & 7/25/08 15:42 & 305.1558 & 13 \\
\hline WVE15861-14 & \begin{tabular}{l|l|}
29195 & WVE15861
\end{tabular} & 7/30/08 11:37 & 305.1674 & 14 \\
\hline WVE15861-15 & 29593 $\mid$ WVE15861 & 8/7/08 17:59 & 305.1843 & 15 \\
\hline WVE15862-1 & \begin{tabular}{l|l|}
26686 & WVE15862
\end{tabular} & 7/2/08 14:07 & 304.9263 & 1 \\
\hline WVE15862-2 & \begin{tabular}{l|l|l|}
26793 & WVE15862 \\
\end{tabular} & 7/3/08 9:58 & 304.9233 & 2 \\
\hline WVE15862-3 & 26897|WVE15862 & $7 / 3 / 08$ 14:39 & 304.9219 & 3 \\
\hline WVE15862-4 & \begin{tabular}{l|l|l|}
27002 & WVE15862 \\
\end{tabular} & 7/7/08 9:34 & 304.9148 & 4 \\
\hline WVE15862-5 & \begin{tabular}{l|l}
27120 & WVE15862 \\
\end{tabular} & 7/7/08 19:56 & 304.5712 & 5 \\
\hline WVE15862-6 & \begin{tabular}{l|l}
27152 & WVE15862
\end{tabular} & $7 / 8 / 08$ 9:50 & 304.6091 & 6 \\
\hline WVE15862-7 & \begin{tabular}{l|l}
27280 & WVE15862 \\
\end{tabular} & $7 / 8 / 0821: 11$ & 304.6216 & 7 \\
\hline WVE15862-8 & \begin{tabular}{l|l|}
27394 & WVE15862
\end{tabular} & 7/9/08 10:19 & 304.6287 & 8 \\
\hline WVE15862-9 & \begin{tabular}{l|l}
27528 & WVE15862
\end{tabular} & 7/10/08 15:48 & 304.6393 & 9 \\
\hline WVE15862-10 & \begin{tabular}{l|l}
27632 & WVE15862 \\
\end{tabular} & $7 / 11 / 08$ 10:09 & 304.6439 & 10 \\
\hline WVE15862-11 & \begin{tabular}{l|l}
27874 & WVE15862
\end{tabular} & 7/14/08 13:09 & 304.6658 & 11 \\
\hline WVE15862-12 & \begin{tabular}{l|l|}
28193 & WVE15862
\end{tabular} & 7/18/08 13:59 & 304.6777 & 12 \\
\hline WVE15862-13 & 28810|WVE15862 & $7 / 25 / 08$ 15:42 & 304.7 & 13 \\
\hline WVE15862-14 & \begin{tabular}{l|l|}
29196 & WVE15862 \\
\end{tabular} & 7/30/08 11:38 & 304.7112 & 14 \\
\hline
\end{tabular}




\begin{tabular}{|c|c|c|c|c|}
\hline WVE15862-15 & \begin{tabular}{l|l|} 
29594 & WVE15862
\end{tabular} & 8/7/08 18:00 & 304.7281 & 15 \\
\hline WVE15863-1 & \begin{tabular}{l|l|}
26687 & WVE15863
\end{tabular} & 7/2/08 14:08 & 305.219 & 1 \\
\hline WVE15863-2 & 26794|WVE15863 & $7 / 3 / 089: 59$ & 305.2181 & 2 \\
\hline WVE15863-3 & 26898|WVE15863 & $7 / 3 / 08$ 14:40 & 305.215 & 3 \\
\hline WVE15863-4 & 27003|WVE15863 & 7/7/08 9:35 & 305.2095 & 4 \\
\hline WVE15863-5 & $27119 \mid$ WVE15863 & 7/7/08 19:56 & 304.8777 & 5 \\
\hline WVE15863-6 & 27153|WVE15863 & 7/8/08 9:51 & 304.9142 & 6 \\
\hline WVE15863-7 & 27281|WVE15863 & 7/8/08 21:12 & 304.9259 & 7 \\
\hline WVE15863-8 & \begin{tabular}{|l|l|}
27395 & WVE15863 \\
\end{tabular} & 7/9/08 10:20 & 304.9358 & 8 \\
\hline WVE15863-9 & \begin{tabular}{|l|l|}
27529 & WVE15863
\end{tabular} & 7/10/08 15:49 & 304.9482 & 9 \\
\hline WVE15863-10 & 27633|WVE15863 & 7/11/08 10:10 & 304.9537 & 10 \\
\hline WVE15863-11 & $27875 \mid$ WVE15863 & 7/14/08 13:10 & 304.9731 & 11 \\
\hline WVE15863-12 & $28194 \mid$ WVE15863 & $7 / 18 / 08$ 14:00 & 304.9876 & 12 \\
\hline WVE15863-13 & $28811 \mid$ WVE15863 & $7 / 25 / 08$ 15:43 & 305.009 & 13 \\
\hline WVE15863-14 & 29197 $\mid$ WVE15863 & 7/30/08 11:39 & 305.0196 & 14 \\
\hline WVE15863-15 & \begin{tabular}{l|l|l|}
29595 & WVE15863
\end{tabular} & 8/7/08 18:01 & 305.037 & 15 \\
\hline WVE15864-1 & 26688|WVE15864 & 7/2/08 14:09 & 308.0564 & 1 \\
\hline WVE15864-2 & 26795|WVE15864 & 7/3/08 10:01 & 308.0533 & 2 \\
\hline WVE15864-3 & 26899|WVE15864 & $7 / 3 / 08$ 14:41 & 308.0515 & 3 \\
\hline WVE15864-4 & \begin{tabular}{l|l|l|}
27004 & WVE15864 \\
\end{tabular} & 7/7/08 9:36 & 308.0464 & 4 \\
\hline WVE15864-5 & \begin{tabular}{l|l|l|}
27118 & WVE15864
\end{tabular} & 7/7/08 19:55 & 307.692 & 5 \\
\hline WVE15864-6 & \begin{tabular}{l|l|l|}
27154 & WVE15864 \\
\end{tabular} & 7/8/08 9:52 & 307.7283 & 6 \\
\hline WVE15864-7 & \begin{tabular}{|l|l|}
27282 & WVE15864
\end{tabular} & $7 / 8 / 0821: 14$ & 307.7375 & 7 \\
\hline WVE15864-8 & \begin{tabular}{l|l|}
27396 & WVE15864
\end{tabular} & 7/9/08 10:20 & 307.7487 & 8 \\
\hline WVE15864-9 & 27530|WVE15864 & $7 / 10 / 08$ 15:50 & 307.7618 & 9 \\
\hline WVE15864-10 & \begin{tabular}{l|l|}
27634 & WVE15864 \\
\end{tabular} & 7/11/08 10:11 & 307.7667 & 10 \\
\hline WVE15864-11 & \begin{tabular}{|l|l|l|}
27876 & WVE15864
\end{tabular} & 7/14/08 13:11 & 307.7857 & 11 \\
\hline WVE15864-12 & \begin{tabular}{l|l|}
28195 & WVE15864
\end{tabular} & 7/18/08 14:01 & 307.801 & 12 \\
\hline WVE15864-13 & \begin{tabular}{l|l|}
28812 & WVE15864
\end{tabular} & $7 / 25 / 08$ 15:45 & 307.8244 & 13 \\
\hline WVE15864-14 & 29198|WVE15864 & 7/30/08 11:39 & 307.8341 & 14 \\
\hline WVE15864-15 & \begin{tabular}{|l|l|}
29596 & WVE15864 \\
\end{tabular} & 8/7/08 18:02 & 307.8527 & 15 \\
\hline WVE15865-1 & 26689|WVE15865 & $7 / 2 / 08$ 14:10 & 302.2394 & 1 \\
\hline WVE15865-2 & 26796|WVE15865 & 7/3/08 10:01 & 302.2351 & 2 \\
\hline WVE15865-3 & 26900|WVE15865 & $7 / 3 / 08$ 14:42 & 302.2343 & 3 \\
\hline WVE15865-4 & \begin{tabular}{|l|l|}
27005 & WVE15865 \\
\end{tabular} & 7/7/08 9:36 & 302.2328 & 4 \\
\hline WVE15865-5 & \begin{tabular}{l|l|l|}
27117 & WVE15865 \\
\end{tabular} & 7/7/08 19:55 & 301.878 & 5 \\
\hline WVE15865-6 & 27155|WVE15865 & 7/8/08 9:52 & 301.9071 & 6 \\
\hline WVE15865-7 & \begin{tabular}{l|l}
27283 & WVE15865
\end{tabular} & $7 / 8 / 0821: 15$ & 301.9181 & 7 \\
\hline WVE15865-8 & \begin{tabular}{|l|l|}
27397 & WVE15865 \\
\end{tabular} & 7/9/08 10:21 & 301.9287 & 8 \\
\hline WVE15865-9 & 27531 $\mid$ WVE15865 & 7/10/08 15:51 & 301.9468 & 9 \\
\hline WVE15865-10 & 27635|WVE15865 & $7 / 11 / 08$ 10:12 & 301.9495 & 10 \\
\hline WVE15865-11 & \begin{tabular}{l|l}
27877 & WVE15865
\end{tabular} & 7/14/08 13:12 & 301.9667 & 11 \\
\hline WVE15865-12 & 28196|WVE15865 & 7/18/08 14:01 & 301.9806 & 12 \\
\hline WVE15865-13 & \begin{tabular}{|l|l|}
28813 & WVE15865
\end{tabular} & $7 / 25 / 08$ 15:45 & 302.0041 & 13 \\
\hline WVE15865-14 & $29199 \mid$ WVE15865 & 7/30/08 11:40 & 302.0142 & 14 \\
\hline WVE15865-15 & \begin{tabular}{|l|l|}
29597 & WVE15865 \\
\end{tabular} & 8/7/08 18:03 & 302.0331 & 15 \\
\hline WVE15866-1 & 26690|WVE15866 & 7/2/08 14:10 & 298.7877 & 1 \\
\hline WVE15866-2 & 26797 WVE15866 & 7/3/08 10:02 & 298.7892 & 2 \\
\hline WVE15866-3 & \begin{tabular}{l|l|}
26901 & WVE15866 \\
\end{tabular} & $7 / 3 / 08$ 14:43 & 298.7868 & 3 \\
\hline
\end{tabular}




\begin{tabular}{|c|c|c|c|c|}
\hline WVE15866-4 & \begin{tabular}{l|l|l|}
27006 & WVE15866
\end{tabular} & 7/7/08 9:37 & 298.7796 & 4 \\
\hline \begin{tabular}{|l|} 
WVE15866-5 \\
\end{tabular} & 27114 $\mid$ WVE15866 & 7/7/08 19:53 & 298.4656 & 5 \\
\hline WVE15866-6 & \begin{tabular}{l|l}
27158 & WVE15866
\end{tabular} & $7 / 8 / 08$ 9:55 & 298.5002 & $\overline{6}$ \\
\hline WVE15866-7 & \begin{tabular}{|l|l|}
27296 & WVE15866
\end{tabular} & 7/8/08 21:34 & 298.5123 & 7 \\
\hline WVE15866-8 & 27398|WVE15866 & $7 / 9 / 08$ 10:22 & 298.5225 & 8 \\
\hline WVE15866-9 & 27522 $\mid$ WVE15866 & $7 / 10 / 08$ 15:43 & 298.5317 & 9 \\
\hline WVE15866-10 & \begin{tabular}{l|l|}
27636 & WVE15866 \\
\end{tabular} & $7 / 11 / 08$ 10:12 & 298.5379 & 10 \\
\hline WVE15866-11 & 27878|WVE15866 & $7 / 14 / 08$ 13:13 & 298.558 & 11 \\
\hline WVE15866-12 & \begin{tabular}{|l|l|}
28197 & WV15866 \\
\end{tabular} & $7 / 18 / 08$ 14:02 & 298.575 & 12 \\
\hline WVE15866-13 & \begin{tabular}{l|l|l|}
28814 & WVE15866 \\
\end{tabular} & $7 / 25 / 08$ 15:46 & 298.5942 & 13 \\
\hline WVE15866-14 & $29200 \mid$ WVE15866 & $7 / 30 / 08$ 11:41 & 298.6049 & 14 \\
\hline WVE15866-15 & \begin{tabular}{l|l|l|}
29598 & WVE15866
\end{tabular} & 8/7/08 18:04 & 298.6221 & 15 \\
\hline WVE15867-1 & \begin{tabular}{|l|l|}
266915867 & WVE158
\end{tabular} & $7 / 2 / 0814: 11$ & 303.7236 & 1 \\
\hline WVE15867-2 & \begin{tabular}{l|l|}
26798 & WVE15867
\end{tabular} & $7 / 3 / 08$ 10:03 & 303.7211 & 2 \\
\hline WVE15867-3 & \begin{tabular}{l|l}
26902 & WVE15867
\end{tabular} & $7 / 3 / 0814: 44$ & 303.7189 & 3 \\
\hline WVE15867-4 & \begin{tabular}{l|l|l|}
27007 & WVE15867
\end{tabular} & 7/7/08 9:38 & 303.7116 & 4 \\
\hline WVE15867-5 & \begin{tabular}{l|l}
27113 & WVE15867
\end{tabular} & $7 / 7 / 08$ 19:52 & 303.3524 & 5 \\
\hline WVE15867-6 & \begin{tabular}{l|l}
27159 & WVE15867
\end{tabular} & $7 / 8 / 08$ 9:56 & 303.3899 & 6 \\
\hline WVE15867-7 & \begin{tabular}{l|l|}
27297 & WVE15867
\end{tabular} & $7 / 8 / 08$ 21:35 & 303.3994 & 7 \\
\hline WVE15867-8 & 27399 $\mid$ WVE15867 & $7 / 9 / 08$ 10:22 & 303.4096 & 8 \\
\hline WVE15867-9 & 27523 WVE15867 & $7 / 10 / 08$ 15:44 & 303.4204 & 9 \\
\hline WVE15867-10 & \begin{tabular}{l|l|l|}
27637 & WVE15867 \\
\end{tabular} & 7/11/08 10:13 & 303.4259 & 10 \\
\hline WVE15867-11 & \begin{tabular}{l|l|l|}
27879 & WVE15867 \\
\end{tabular} & $7 / 14 / 08$ 13:14 & 303.4434 & 11 \\
\hline WVE15867-12 & \begin{tabular}{l|l|l|}
28198 & WVE15867 \\
\end{tabular} & 7/18/08 14:03 & 303.4624 & 12 \\
\hline WVE15867-13 & \begin{tabular}{l|l|l}
28815 & WVE15867
\end{tabular} & $7 / 25 / 08$ 15:47 & 303.4813 & 13 \\
\hline WVE15867-14 & \begin{tabular}{l|l|l|}
29201 & WVE15867
\end{tabular} & 7/30/08 11:41 & 303.4924 & 14 \\
\hline WVE15867-15 & \begin{tabular}{l|l|l}
29599 & WVE15867 \\
\end{tabular} & 8/7/08 18:05 & 303.5084 & 15 \\
\hline WVE15868-1 & \begin{tabular}{l|l|} 
26692 & WVE15868
\end{tabular} & $7 / 2 / 08$ 14:12 & 301.3992 & 1 \\
\hline WVE15868-2 & 26799 WVE15868 & $7 / 3 / 08$ 10:04 & 301.3987 & 2 \\
\hline WVE15868-3 & \begin{tabular}{l|l|l|}
26903 & WVE15868 \\
\end{tabular} & 7/3/08 14:44 & 301.3981 & 3 \\
\hline WVE15868-4 & \begin{tabular}{l|l|}
27008 & WVE15868
\end{tabular} & 7/7/08 9:38 & 301.3922 & 4 \\
\hline WVE15868-5 & \begin{tabular}{l|l|l|}
27112 & WVE15868 \\
\end{tabular} & 7/7/08 19:52 & 301.0618 & 5 \\
\hline WVE15868-6 & 27160 $\mid$ WVE15868 & $7 / 8 / 08$ 9:57 & 301.0988 & 6 \\
\hline WVE15868-7 & \begin{tabular}{l|l|l|}
27298 & WVE15868 \\
\end{tabular} & 7/8/08 21:36 & 301.1082 & 7 \\
\hline WVE15868-8 & \begin{tabular}{l|l}
27400 & WVE15868
\end{tabular} & $7 / 9 / 08$ 10:23 & 301.118 & 8 \\
\hline WVE15868-9 & \begin{tabular}{l|l|l|}
27524 & WVE15868 \\
\end{tabular} & $7 / 10 / 08$ 15:45 & 301.1301 & 9 \\
\hline WVE15868-10 & \begin{tabular}{l|l|l|}
27638 & WVE15868 \\
\end{tabular} & 7/11/08 10:14 & 301.1359 & 10 \\
\hline WVE15868-11 & \begin{tabular}{l|l|l|}
27880 & WVE15868 \\
\end{tabular} & $7 / 14 / 08$ 13:15 & 301.1581 & 11 \\
\hline WVE15868-12 & 28199 WVE15868 & 7/18/08 14:04 & 301.1743 & 12 \\
\hline WVE15868-13 & \begin{tabular}{l|l|l|}
28816 & WVE15868
\end{tabular} & $7 / 25 / 08$ 15:48 & 301.1927 & 13 \\
\hline WVE15868-14 & \begin{tabular}{l|l|l}
29202 & WVE15868 \\
\end{tabular} & 7/30/08 11:42 & 301.2028 & 14 \\
\hline WVE15868-15 & \begin{tabular}{l|l|l}
29600 & WVE15868
\end{tabular} & 8/7/08 18:05 & 301.2208 & 15 \\
\hline WVE15869-1 & \begin{tabular}{l|l}
26693 & WVE15869
\end{tabular} & $7 / 2 / 08$ 14:13 & 304.6352 & 1 \\
\hline WVE15869-2 & 26800 $\mid$ WVE15869 & $7 / 3 / 08$ 10:05 & 304.6313 & 2 \\
\hline WVE15869-3 & \begin{tabular}{l|l|l|}
26904 & WVE15869 \\
\end{tabular} & $7 / 3 / 08$ 14:45 & 304.6303 & 3 \\
\hline WVE15869-4 & 27009 WVE15869 & 7/7/08 9:39 & 304.623 & 4 \\
\hline WVE15869-5 & \begin{tabular}{|l|l|}
2711 & WVE15869 \\
\end{tabular} & 7/7/08 19:51 & 304.3037 & 5 \\
\hline WVE15869-6 & \begin{tabular}{l|l|l|}
27161 & WVE15869 \\
\end{tabular} & 7/8/08 9:57 & 304.3393 & 6 \\
\hline WVE15869-7 & 27299 WVE15869 & $7 / 8 / 0821: 38$ & 304.3508 & 7 \\
\hline
\end{tabular}




\begin{tabular}{|c|c|c|c|c|}
\hline WVE15869-8 & \begin{tabular}{l|l|l|}
27401 & WVE15869 \\
\end{tabular} & 7/9/08 10:24 & 304.3597 & 8 \\
\hline WVE15869-9 & 27525|WVE15869 & 7/10/08 15:45 & 304.3736 & 9 \\
\hline WVE15869-10 & \begin{tabular}{|l|l|}
27639 & WVE15869
\end{tabular} & 7/11/08 10:14 & 304.3774 & 10 \\
\hline WVE15869-11 & \begin{tabular}{l|l|}
27881 & WVE15869 \\
\end{tabular} & 7/14/08 13:16 & 304.3957 & 11 \\
\hline WVE15869-12 & \begin{tabular}{|l|l|}
28200 & WVE15869
\end{tabular} & 7/18/08 14:05 & 304.4107 & 12 \\
\hline WVE15869-13 & \begin{tabular}{l|l|l|}
28817 & WVE15869 \\
\end{tabular} & $7 / 25 / 08$ 15:49 & 304.4329 & 13 \\
\hline WVE15869-14 & $29203 \mid$ WVE15869 & $7 / 30 / 08$ 11:43 & 304.445 & 14 \\
\hline WVE15869-15 & \begin{tabular}{l|l|l|}
29601 & WVE15869 \\
\end{tabular} & 8/7/08 18:06 & 304.4625 & 15 \\
\hline WVE15870-1 & 26694|WVE15870 & 7/2/08 14:14 & 306.4046 & 1 \\
\hline WVE15870-2 & \begin{tabular}{l|l|}
26801 & WVE15870 \\
\end{tabular} & 7/3/08 10:05 & 306.4021 & 2 \\
\hline WVE15870-3 & 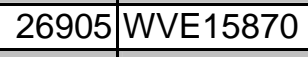 & 7/3/08 14:46 & 306.4 & 3 \\
\hline WVE15870-4 & \begin{tabular}{|l|l|}
27010 & WVE15870 \\
\end{tabular} & 7/7/08 9:39 & 306.3941 & 4 \\
\hline WVE15870-5 & 27110 $\mid$ WVE15870 & 7/7/08 19:50 & 306.0669 & 5 \\
\hline WVE15870-6 & \begin{tabular}{l|l|l|}
27162 & WVE15870 \\
\end{tabular} & 7/8/08 9:58 & 306.1064 & 6 \\
\hline WVE15870-7 & $27300 \mid$ WVE15870 & 7/8/08 21:38 & 306.1157 & 7 \\
\hline WVE15870-8 & \begin{tabular}{l|l|l}
27402 & WVE15870 \\
\end{tabular} & 7/9/08 10:25 & 306.1238 & 8 \\
\hline WVE15870-9 & 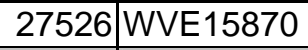 & 7/10/08 15:46 & 306.1369 & 9 \\
\hline WVE15870-10 & \begin{tabular}{l|l|l}
27640 & WVE15870 \\
\end{tabular} & 7/11/08 10:15 & 306.1391 & 10 \\
\hline WVE15870-11 & \begin{tabular}{l|l|l|}
27882 & WVE15870 \\
\end{tabular} & 7/14/08 13:17 & 306.1572 & 11 \\
\hline WVE15870-12 & \begin{tabular}{l|l|l|}
28201 & WVE15870 \\
\end{tabular} & $7 / 18 / 08$ 14:05 & 306.1722 & 12 \\
\hline WVE15870-13 & 28818|WVE15870 & $7 / 25 / 08$ 15:50 & 306.1933 & 13 \\
\hline WVE15870-14 & \begin{tabular}{l|l|l|}
29204 & WVE15870 \\
\end{tabular} & $7 / 30 / 08$ 11:44 & 306.204 & 14 \\
\hline WVE15870-15 & \begin{tabular}{l|l|l|}
29602 & WVE15870 \\
\end{tabular} & 8/7/08 18:07 & 306.2242 & 15 \\
\hline WVE15871-1 & 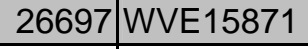 & 7/2/08 14:18 & 429.9967 & 1 \\
\hline WVE15871-2 & \begin{tabular}{l|l|}
26804 & WVE15871 \\
\end{tabular} & 7/3/08 10:10 & 429.9874 & 2 \\
\hline WVE15871-3 & \begin{tabular}{l|l|l}
26907 & WVE15871
\end{tabular} & 7/3/08 14:51 & 429.988 & 3 \\
\hline WVE15871-4 & $27014 \mid$ WVE15871 & 7/7/08 9:44 & 429.9843 & 4 \\
\hline WVE15871-5 & 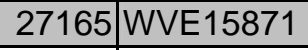 & 7/8/08 10:01 & 429.9862 & 5 \\
\hline WVE15871-6 & 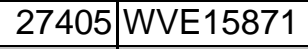 & 7/9/08 10:29 & 429.9892 & 6 \\
\hline WVE15871-7 & 27539 WVE15871 & 7/10/08 16:00 & 429.9861 & 7 \\
\hline WVE15871-8 & $27643 \mid$ WVE15871 & 7/11/08 10:19 & 429.9869 & 8 \\
\hline WVE15871-9 & \begin{tabular}{|l|l|}
27885 & WVE15871 \\
\end{tabular} & 7/14/08 13:21 & 429.9888 & 9 \\
\hline WVE15871-10 & \begin{tabular}{l|l|l|}
28204 & WVE15871 \\
\end{tabular} & 7/18/08 14:09 & 429.9919 & 10 \\
\hline WVE15871-11 & \begin{tabular}{l|l|l|}
28821 & WVE15871 \\
\end{tabular} & $7 / 25 / 08$ 15:53 & 429.9963 & 11 \\
\hline WVE15871-12 & \begin{tabular}{l|l|l|}
29207 & WVE15871 \\
\end{tabular} & 7/30/08 11:47 & 430.0036 & 12 \\
\hline WVE15871-13 & \begin{tabular}{l|l|l}
29605 & WVE15871 \\
\end{tabular} & 8/7/08 18:52 & 430.0118 & 13 \\
\hline WVE15872-1 & 26698 $\mid$ WVE15872 & $7 / 2 / 0814: 19$ & 429.8105 & 1 \\
\hline WVE15872-2 & $26805 \mid$ WVE15872 & 7/3/08 10:11 & 429.8114 & 2 \\
\hline WVE15872-3 & 26908 $\mid$ WVE15872 & 7/3/08 14:52 & 429.8106 & 3 \\
\hline WVE15872-4 & 27015 WVE15872 & 7/7/08 9:45 & 429.8046 & 4 \\
\hline WVE15872-5 & 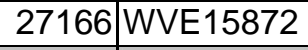 & 7/8/08 10:02 & 429.8038 & 5 \\
\hline WVE15872-6 & 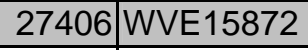 & 7/9/08 10:29 & 429.808 & 6 \\
\hline WVE15872-7 & 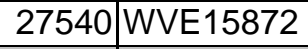 & 7/10/08 16:01 & 429.8075 & 7 \\
\hline WVE15872-8 & 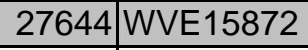 & 7/11/08 10:20 & 429.8093 & 8 \\
\hline WVE15872-9 & \begin{tabular}{l|l|l|}
27886 & WVE15872 \\
\end{tabular} & 7/14/08 13:22 & 429.8134 & 9 \\
\hline WVE15872-10 & 28205 WVE15872 & 7/18/08 14:10 & 429.8119 & 10 \\
\hline WVE15872-11 & \begin{tabular}{l|l}
28822 & WVE15872 \\
\end{tabular} & $7 / 25 / 08$ 15:54 & 429.8138 & 11 \\
\hline WVE15872-12 & 29208 WVE15872 & 7/30/08 11:48 & 429.8176 & 12 \\
\hline WVE15872-13 & 29606|WVE15872 & 8/7/08 18:52 & 429.8258 & 13 \\
\hline
\end{tabular}




\begin{tabular}{|c|c|c|c|c|}
\hline WVE15873-1 & 26699|WVE15873 & $7 / 2 / 08$ 14:20 & 444.196 & 1 \\
\hline WVE15873-2 & 26806|WVE15873 & 7/3/08 10:13 & 444.1894 & 2 \\
\hline WVE15873-3 & 26909 WVE15873 & 7/3/08 14:53 & 444.189 & 3 \\
\hline WVE15873-4 & \begin{tabular}{|l|l|}
27016 & WVE15873 \\
\end{tabular} & 7/7/08 9:46 & 444.1817 & 4 \\
\hline WVE15873-5 & 27167|WVE15873 & 7/8/08 10:03 & 444.1861 & 5 \\
\hline WVE15873-6 & 27407 $\mid$ WVE15873 & 7/9/08 10:30 & 444.1856 & 6 \\
\hline WVE15873-7 & 27541|WVE15873 & $7 / 10 / 08$ 16:02 & 444.1875 & 7 \\
\hline WVE15873-8 & $27645 \mid$ WVE15873 & 7/11/08 10:20 & 444.1858 & 8 \\
\hline WVE15873-9 & 27887 $\mid$ WVE15873 & 7/14/08 13:23 & 444.1895 & 9 \\
\hline WVE15873-10 & \begin{tabular}{|l|l|}
28206 & WVE15873 \\
\end{tabular} & 7/18/08 14:11 & 444.1906 & 10 \\
\hline WVE15873-11 & 28823 $\mid$ WVE15873 & $7 / 25 / 08$ 15:55 & 444.196 & 11 \\
\hline WVE15873-12 & 29209|WVE15873 & 7/30/08 11:48 & 444.1987 & 12 \\
\hline WVE15873-13 & 29607 $\mid$ WVE15873 & 8/7/08 18:54 & 444.2055 & 13 \\
\hline WVE15874-1 & 26700 $\mid$ WVE15874 & $7 / 2 / 0814: 21$ & 431.1215 & 1 \\
\hline WVE15874-2 & \begin{tabular}{|l|l|}
26807 & WVE15874 \\
\end{tabular} & 7/3/08 10:13 & 431.1183 & 2 \\
\hline WVE15874-3 & 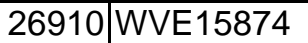 & 7/3/08 14:53 & 431.1172 & 3 \\
\hline WVE15874-4 & \begin{tabular}{l|l|l|}
27017 & WVE15874 \\
\end{tabular} & 7/7/08 9:47 & 431.111 & 4 \\
\hline WVE15874-5 & \begin{tabular}{|l|l|}
27168 & WVE15874 \\
\end{tabular} & 7/8/08 10:04 & 431.1114 & 5 \\
\hline WVE15874-6 & \begin{tabular}{|l|l|}
27408 & WVE15874 \\
\end{tabular} & 7/9/08 10:31 & 431.1165 & 6 \\
\hline WVE15874-7 & \begin{tabular}{l|l|l|}
27542 & WVE15874 \\
\end{tabular} & 7/10/08 16:03 & 431.1136 & 7 \\
\hline WVE15874-8 & \begin{tabular}{|l|l|}
27646 & WVE15874 \\
\end{tabular} & 7/11/08 10:21 & 431.1145 & 8 \\
\hline WVE15874-9 & \begin{tabular}{l|l|l|}
27888 & WVE15874 \\
\end{tabular} & 7/14/08 13:24 & 431.1161 & 9 \\
\hline WVE15874-10 & \begin{tabular}{l|l|l|}
28207 & WVE15874 \\
\end{tabular} & 7/18/08 14:12 & 431.1162 & 10 \\
\hline WVE15874-11 & \begin{tabular}{|l|l|}
28824 & WVE15874 \\
\end{tabular} & $7 / 25 / 08$ 15:56 & 431.1265 & 11 \\
\hline WVE15874-12 & 29210 $\mid$ WVE15874 & $7 / 30 / 08$ 11:49 & 431.1296 & 12 \\
\hline WVE15874-13 & \begin{tabular}{|l|l|}
29608 & WVE15874 \\
\end{tabular} & 8/7/08 18:54 & 431.1336 & 13 \\
\hline WVE15875-1 & 26701 $\mid$ WVE15875 & $7 / 2 / 0814: 22$ & 434.3362 & 1 \\
\hline WVE15875-2 & 26808 $\mid$ WVE15875 & 7/3/08 10:14 & 434.3228 & 2 \\
\hline WVE15875-3 & 26911 WVE15875 & 7/3/08 14:54 & 434.3384 & 3 \\
\hline WVE15875-4 & 27018|WVE15875 & 7/7/08 9:48 & 434.3078 & 4 \\
\hline WVE15875-5 & 27169 WVE15875 & 7/8/08 10:05 & 434.3081 & 5 \\
\hline WVE15875-6 & 27409 WVE15875 & 7/9/08 10:32 & 434.3109 & 6 \\
\hline WVE15875-7 & 27543 $\mid$ WVE15875 & 7/10/08 16:04 & 434.3147 & 7 \\
\hline WVE15875-8 & 27647 $\mid$ WVE15875 & 7/11/08 10:22 & 434.3202 & 8 \\
\hline WVE15875-9 & 27889 WVE15875 & $7 / 14 / 08$ 13:25 & 434.3327 & 9 \\
\hline WVE15875-10 & 28208|WVE15875 & $7 / 18 / 08$ 14:14 & 434.3319 & 10 \\
\hline WVE15875-11 & \begin{tabular}{|l|l|}
28825 & WVE15875 \\
\end{tabular} & $7 / 25 / 08$ 15:57 & 434.3215 & 11 \\
\hline WVE15875-12 & 29211 $\mid$ WVE15875 & $7 / 30 / 08$ 11:50 & 434.3279 & 12 \\
\hline WVE15875-13 & 29609 WVE15875 & 8/7/08 18:55 & 434.3313 & 13 \\
\hline WVE15876-1 & \begin{tabular}{|l|l|}
26702 & WVE15876 \\
\end{tabular} & $7 / 2 / 0814: 23$ & 429.5883 & 1 \\
\hline WVE15876-2 & 26809 WVE15876 & $7 / 3 / 08$ 10:16 & 429.5814 & 2 \\
\hline WVE15876-3 & 26912 WVE15876 & 7/3/08 14:55 & 429.5837 & 3 \\
\hline WVE15876-4 & 27019 WVE15876 & 7/7/08 9:49 & 429.5757 & 4 \\
\hline WVE15876-5 & \begin{tabular}{|l|l|l|}
2720 & WVE15876 \\
\end{tabular} & 7/8/08 14:50 & 429.5915 & 5 \\
\hline WVE15876-6 & 27291 WVE15876 & $7 / 8 / 0821: 28$ & 429.5924 & 6 \\
\hline WVE15876-7 & \begin{tabular}{|l|l|}
27410 & WVE15876 \\
\end{tabular} & 7/9/08 10:33 & 429.5945 & 7 \\
\hline WVE15876-8 & \begin{tabular}{l|l|l|}
27534 & WVE15876 \\
\end{tabular} & 7/10/08 15:55 & 429.5976 & 8 \\
\hline WVE15876-9 & \begin{tabular}{l|l|l|}
27648 & WVE15876 \\
\end{tabular} & 7/11/08 10:23 & 429.5895 & 9 \\
\hline WVE15876-10 & \begin{tabular}{l|l|l|}
27890 & WVE15876 \\
\end{tabular} & 7/14/08 13:26 & 429.5919 & 10 \\
\hline
\end{tabular}




\begin{tabular}{|c|c|c|c|c|}
\hline WVE15876-11 & \begin{tabular}{l|l|l|}
28209 & WVE15876
\end{tabular} & $7 / 18 / 08$ 14:15 & 429.5957 & 11 \\
\hline WVE15876-12 & \begin{tabular}{l|l|} 
28826 & WVE15876 \\
\end{tabular} & 7/25/08 15:58 & 429.5947 & 12 \\
\hline WVE15876-13 & \begin{tabular}{l|l|}
29212 & WVE15876
\end{tabular} & 7/30/08 11:51 & 429.6052 & 13 \\
\hline WVE15876-14 & 29610 $\mid$ WVE15876 & $8 / 7 / 08$ 18:56 & 429.6103 & 14 \\
\hline WVE15877-1 & 26703|WVE15877 & $7 / 2 / 08$ 14:23 & 432.0483 & 1 \\
\hline WVE15877-2 & 26810|WVE15877 & $7 / 3 / 08$ 10:16 & 432.0427 & 2 \\
\hline WVE15877-3 & 26913|WVE15877 & 7/3/08 14:56 & 432.0469 & 3 \\
\hline WVE15877-4 & \begin{tabular}{|l|l|l}
27020 & WVE15877
\end{tabular} & 7/7/08 9:50 & 432.0434 & 4 \\
\hline WVE15877-5 & \begin{tabular}{|l|l|}
27221 & WVE15877 \\
\end{tabular} & $7 / 8 / 08$ 14:51 & 432.0563 & 5 \\
\hline WVE15877-6 & \begin{tabular}{|l|l|}
27292 & WVE15877
\end{tabular} & $7 / 8 / 0821: 30$ & 432.0498 & 6 \\
\hline WVE15877-7 & 27411 $\mid$ WVE15877 & 7/9/08 10:34 & 432.0505 & 7 \\
\hline WVE15877-8 & \begin{tabular}{l|l|l}
27535 & WVE15877
\end{tabular} & $7 / 10 / 08$ 15:56 & 432.0512 & 8 \\
\hline WVE15877-9 & 27649 WVE15877 & $7 / 11 / 08$ 10:24 & 432.047 & 9 \\
\hline WVE15877-10 & \begin{tabular}{l|l|l}
27891 & WVE15877
\end{tabular} & $7 / 14 / 08$ 13:28 & 432.0438 & 10 \\
\hline WVE15877-11 & 28210|WVE15877 & 7/18/08 14:17 & 432.0472 & 11 \\
\hline WVE15877-12 & \begin{tabular}{l|l|}
28827 & WVE15877 \\
\end{tabular} & 7/25/08 15:59 & 432.048 & 12 \\
\hline WVE15877-13 & 29213|WVE15877 & 7/30/08 11:52 & 432.0577 & 13 \\
\hline WVE15877-14 & 29611|WVE15877 & 8/7/08 18:57| & 432.0597 & 14 \\
\hline WVE15878-1 & 26704|WVE15878 & $7 / 2 / 08$ 14:24 & 420.2935 & 1 \\
\hline WVE15878-2 & \begin{tabular}{|l|l|}
26811 & WVE15878 \\
\end{tabular} & 7/3/08 10:18 & 420.2898 & 2 \\
\hline WVE15878-3 & 26914|WVE15878 & 7/3/08 14:57 & 420.2884 & 3 \\
\hline WVE15878-4 & 27021 $\mid$ WVE15878 & 7/7/08 9:51 & 420.2841 & 4 \\
\hline WVE15878-5 & \begin{tabular}{|l|l|}
27222 & WVE15878
\end{tabular} & $7 / 8 / 08$ 14:52 & 420.3018 & 5 \\
\hline WVE15878-6 & \begin{tabular}{l|l}
27293 & WVE15878
\end{tabular} & 7/8/08 21:31 & 420.2979 & 6 \\
\hline WVE15878-7 & 27412 $\mid$ WVE15878 & 7/9/08 10:34 & 420.2946 & 7 \\
\hline WVE15878-8 & \begin{tabular}{|l|l|}
27536 & WVE15878 \\
\end{tabular} & 7/10/08 15:57 & 420.2918 & 8 \\
\hline WVE15878-9 & 27650|WVE15878 & $7 / 11 / 08$ 10:25 & 420.2902 & 9 \\
\hline WVE15878-10 & \begin{tabular}{l|l|}
27892 & WVE15878
\end{tabular} & 7/14/08 13:29 & 420.2929 & 10 \\
\hline WVE15878-11 & 28211 $\mid$ WVE15878 & 7/18/08 14:18 & 420.2919 & 11 \\
\hline WVE15878-12 & 28828 $\mid$ WVE15878 & $7 / 25 / 08$ 16:00 & 420.2955 & 12 \\
\hline WVE15878-13 & 29214|WVE15878 & 7/30/08 11:53 & 420.3044 & 13 \\
\hline WVE15878-14 & \begin{tabular}{l|l|l|}
29612 & WVE15878
\end{tabular} & 8/7/08 18:57 & 420.3078 & 14 \\
\hline WVE15879-1 & 26705|WVE15879 & $7 / 2 / 08$ 14:25 & 429.0905 & 1 \\
\hline WVE15879-2 & \begin{tabular}{|l|l|}
26812 & WVE15879
\end{tabular} & 7/3/08 10:18 & 429.0895 & 2 \\
\hline WVE15879-3 & \begin{tabular}{|l|l|}
26915 & WVE15879 \\
\end{tabular} & $7 / 3 / 08$ 14:58 & 429.0869 & 3 \\
\hline WVE15879-4 & \begin{tabular}{l|l|}
27022 & WVE15879
\end{tabular} & 7/7/08 9:52 & 428.9944 & 4 \\
\hline WVE15879-5 & 27223 $\mid$ WVE15879 & 7/8/08 14:53 & 429.0134 & 5 \\
\hline WVE15879-6 & \begin{tabular}{l|l|l|}
27294 & WVE15879
\end{tabular} & $7 / 8 / 08$ 21:32 & 429.0066 & 6 \\
\hline WVE15879-7 & \begin{tabular}{|l|l|}
27413 & WVE15879 \\
\end{tabular} & 7/9/08 10:36 & 429.0022 & 7 \\
\hline WVE15879-8 & 27537 $\mid$ WVE15879 & 7/10/08 15:58 & 428.9997 & 8 \\
\hline WVE15879-9 & 27651 WVE15879 & $7 / 11 / 08$ 10:25 & 428.9987 & 9 \\
\hline WVE15879-10 & 27893 $\mid$ WVE15879 & 7/14/08 13:30 & 429.0016 & 10 \\
\hline WVE15879-11 & \begin{tabular}{l|l|l|}
28212 & WVE15879
\end{tabular} & 7/18/08 14:19 & 428.999 & 11 \\
\hline WVE15879-12 & 28829 WVE15879 & $7 / 25 / 08$ 16:01 & 429.0008 & 12 \\
\hline WVE15879-13 & 29215|WVE15879 & $7 / 30 / 08$ 11:54 & 429.0086 & 13 \\
\hline WVE15879-14 & \begin{tabular}{|l|l|}
29613 & WVE15879 \\
\end{tabular} & 8/7/08 18:58 & 429.0088 & 14 \\
\hline WVE15880-1 & \begin{tabular}{l|l|}
26706 & WVE15880
\end{tabular} & $7 / 2 / 08$ 14:26 & 434.1802 & 1 \\
\hline WVE15880-2 & 26813 $\mid$ WVE15880 & 7/3/08 10:19 & 434.1631 & 2 \\
\hline WVE15880-3 & \begin{tabular}{l|l|}
26916 & WVE15880 \\
\end{tabular} & 7/3/08 14:59 & 434.1629 & 3 \\
\hline
\end{tabular}




\begin{tabular}{|c|c|c|c|c|}
\hline WVE15880-4 & 27023 $\mid$ WVE15880 & 7/7/08 9:53 & 434.1588 & 4 \\
\hline WVE15880-5 & 27224 $\mid$ WVE15880 & $7 / 8 / 08$ 14:54 & 434.1725 & 5 \\
\hline WVE15880-6 & \begin{tabular}{l|l|l|}
27295 & WVE15880
\end{tabular} & $7 / 8 / 0821: 33$ & 434.1657 & 6 \\
\hline WVE15880-7 & \begin{tabular}{l|l|}
27414 & WVE15880
\end{tabular} & 7/9/08 10:36 & 434.1651 & 7 \\
\hline WVE15880-8 & \begin{tabular}{l|l|}
27538 & WVE15880
\end{tabular} & 7/10/08 15:59 & 434.1615 & 8 \\
\hline WVE15880-9 & 27652 $\mid$ WVE15880 & 7/11/08 10:27 & 434.1599 & 9 \\
\hline WVE15880-10 & 27894|WVE15880 & 7/14/08 13:31 & 434.1618 & 10 \\
\hline WVE15880-11 & \begin{tabular}{l|l|}
28213 & WVE15880
\end{tabular} & $7 / 18 / 08$ 14:21 & 434.1629 & 11 \\
\hline WVE15880-12 & \begin{tabular}{l|l|l|}
28830 & WVE15880 \\
\end{tabular} & 7/25/08 16:02 & 434.1661 & 12 \\
\hline WVE15880-13 & \begin{tabular}{l|l|}
29216 & WVE15880
\end{tabular} & $7 / 30 / 08$ 11:55 & 434.1765 & 13 \\
\hline WVE15880-14 & \begin{tabular}{l|l|l|}
29614 & WVE15880 \\
\end{tabular} & 8/7/08 18:59 & 434.1764 & 14 \\
\hline WVE15881-1 & $26709 \mid$ WVE15881 & $7 / 2 / 08$ 14:31 & 430.0748 & 1 \\
\hline WVE15881-2 & \begin{tabular}{l|l|l}
26816 & WVE15881 \\
\end{tabular} & $7 / 3 / 08$ 10:24 & 430.0706 & 2 \\
\hline WVE15881-3 & 26923|WVE15881 & $7 / 3 / 08$ 15:10 & 430.0679 & 3 \\
\hline WVE15881-4 & \begin{tabular}{l|l|}
27026 & WVE15881 \\
\end{tabular} & 7/7/08 9:57 & 430.0687 & 4 \\
\hline WVE15881-5 & \begin{tabular}{l|l|}
27227 & WVE15881 \\
\end{tabular} & $7 / 8 / 08$ 14:56 & 429.9324 & 5 \\
\hline WVE15881-6 & \begin{tabular}{l|l|}
27308 & WVE15881 \\
\end{tabular} & $7 / 8 / 0821: 51$ & 429.9324 & 6 \\
\hline WVE15881-7 & \begin{tabular}{l|l|l|}
27417 & WVE15881
\end{tabular} & $7 / 9 / 08$ 10:39 & 429.9408 & 7 \\
\hline WVE15881-8 & \begin{tabular}{l|l|l}
27546 & WVE15881 \\
\end{tabular} & $7 / 10 / 08$ 16:07 & 429.9527 & 8 \\
\hline WVE15881-9 & \begin{tabular}{l|l|l|}
27655 & WVE15881 \\
\end{tabular} & 7/11/08 10:30 & 429.9511 & 9 \\
\hline WVE15881-10 & \begin{tabular}{l|l|l|}
27897 & WVE15881 \\
\end{tabular} & 7/14/08 13:36 & 429.9639 & 10 \\
\hline WVE15881-11 & \begin{tabular}{l|l|}
28216 & WVE15881 \\
\end{tabular} & $7 / 18 / 08$ 14:25 & 429.9683 & 11 \\
\hline WVE15881-12 & 28833 WVE15881 & 7/25/08 16:06 & 429.9856 & 12 \\
\hline WVE15881-13 & $29219 \mid$ WVE15881 & 7/30/08 11:58 & 430.0002 & 13 \\
\hline WVE15881-14 & \begin{tabular}{l|l|}
29617 & WVE15881 \\
\end{tabular} & 8/7/08 19:01 & 430.0083 & 14 \\
\hline WVE15882-1 & \begin{tabular}{l|l|}
26710 & WVE15882 \\
\end{tabular} & 7/2/08 14:32 & 442.7305 & 1 \\
\hline WVE15882-2 & \begin{tabular}{l|l|l|}
26817 & WVE15882 \\
\end{tabular} & $7 / 3 / 08$ 10:25 & 442.7214 & 2 \\
\hline WVE15882-3 & \begin{tabular}{l|l|l} 
26922 & WVE15882
\end{tabular} & $7 / 3 / 08$ 15:09 & 442.7246 & 3 \\
\hline WVE15882-4 & \begin{tabular}{l|l}
27027 & WVE15882
\end{tabular} & 7/7/08 9:58 & 442.7359 & 4 \\
\hline WVE15882-5 & \begin{tabular}{|l|l|}
27228 & WVE15882
\end{tabular} & $7 / 8 / 08$ 14:57 & 442.5523 & 5 \\
\hline WVE15882-6 & \begin{tabular}{l|l|l}
27309 & WVE15882 \\
\end{tabular} & $7 / 8 / 08$ 21:52 & 442.5525 & 6 \\
\hline WVE15882-7 & 27418 $\mid$ WVE15882 & $7 / 9 / 08$ 10:40 & 442.5641 & 7 \\
\hline WVE15882-8 & \begin{tabular}{l|l|}
27547 & WVE15882 \\
\end{tabular} & 7/10/08 16:08 & 442.5762 & 8 \\
\hline WVE15882-9 & \begin{tabular}{l|l}
27656 & WVE15882
\end{tabular} & 7/11/08 10:31 & 442.583 & 9 \\
\hline WVE15882-10 & \begin{tabular}{l|l}
27898 & WVE15882
\end{tabular} & 7/14/08 13:37 & 442.6015 & 10 \\
\hline WVE15882-11 & \begin{tabular}{l|l|l|}
28217 & WVE15882
\end{tabular} & 7/18/08 14:27 & 442.6049 & 11 \\
\hline WVE15882-12 & \begin{tabular}{l|l|l|}
28834 & WVE15882 \\
\end{tabular} & 7/25/08 16:07 & 442.6265 & 12 \\
\hline WVE15882-13 & 29220|WVE15882 & 7/30/08 11:59 & 442.6425 & 13 \\
\hline WVE15882-14 & \begin{tabular}{l|l|}
29618 & WVE15882 \\
\end{tabular} & 8/7/08 19:02 & 442.6518 & 14 \\
\hline WVE15883-1 & \begin{tabular}{l|l|l|}
26711 & WVE15883 \\
\end{tabular} & 7/2/08 14:33 & 434.6571 & 1 \\
\hline WVE15883-2 & \begin{tabular}{l|l|}
26818 & WVE15883 \\
\end{tabular} & $7 / 3 / 08$ 10:26 & 434.6507 & 2 \\
\hline WVE15883-3 & \begin{tabular}{l|l}
26921 & WVE15883
\end{tabular} & 7/3/08 15:08 & 434.6538 & 3 \\
\hline WVE15883-4 & \begin{tabular}{|l|l|}
27028 & WVE15883
\end{tabular} & 7/7/08 9:58 & 434.6513 & 4 \\
\hline WVE15883-5 & 27229 WVE15883 & 7/8/08 14:58 & 434.4381 & 5 \\
\hline WVE15883-6 & 27310|WVE15883 & 7/8/08 21:53 & 434.4417 & 6 \\
\hline WVE15883-7 & 27419|WVE15883 & $7 / 9 / 08$ 10:41 & 434.4587 & 7 \\
\hline WVE15883-8 & \begin{tabular}{l|l|}
27548 & WVE15883 \\
\end{tabular} & 7/10/08 16:09 & 434.4679 & 8 \\
\hline WVE15883-9 & \begin{tabular}{l|l|l|}
27657 & WVE15883 \\
\end{tabular} & 7/11/08 10:31 & 434.4675 & 9 \\
\hline WVE15883-10 & \begin{tabular}{l|l|}
27899 & WVE15883 \\
\end{tabular} & 7/14/08 13:38 & 434.4849 & 10 \\
\hline
\end{tabular}




\begin{tabular}{|c|c|c|c|c|}
\hline WVE15883-11 & 28218 $\mid$ WVE15883 & 7/18/08 14:28 & 434.4957 & 11 \\
\hline WVE15883-12 & 28835|WVE15883 & 7/25/08 16:08 & 434.5084 & 12 \\
\hline WVE15883-13 & \begin{tabular}{|l|l|}
$29221 \mid W V E 15883$ \\
\end{tabular} & 7/30/08 12:00 & 434.5225 & 13 \\
\hline WVE15883-14 & 29619 WVE15883 & 8/7/08 19:03 & 434.5328 & 14 \\
\hline WVE15884-1 & \begin{tabular}{l|l|l|}
26712 & WVE15884 \\
\end{tabular} & 7/2/08 14:34 & 436.7236 & 1 \\
\hline WVE15884-2 & \begin{tabular}{l|l|l|}
26819 & WVE15884 \\
\end{tabular} & $7 / 3 / 08$ 10:27 & 436.7197 & 2 \\
\hline WVE15884-3 & \begin{tabular}{|l|l|}
26920 & WVE15884 \\
\end{tabular} & 7/3/08 15:07 & 436.7193 & 3 \\
\hline WVE15884-4 & \begin{tabular}{l|l|l|}
27029 & WVE15884 \\
\end{tabular} & 7/7/08 9:59 & 436.715 & 4 \\
\hline WVE15884-5 & 27230|WVE15884 & 7/8/08 15:01 & 436.5385 & 5 \\
\hline WVE15884-6 & \begin{tabular}{l|l|}
27311 & WVE15884 \\
\end{tabular} & $7 / 8 / 0821: 54$ & 436.5398 & 6 \\
\hline WVE15884-7 & \begin{tabular}{|l|l|}
27420 & WVE15884 \\
\end{tabular} & 7/9/08 10:42 & 436.5533 & 7 \\
\hline WVE15884-8 & \begin{tabular}{|l|l|}
27549 & WVE15884
\end{tabular} & 7/10/08 16:10 & 436.5638 & 8 \\
\hline WVE15884-9 & \begin{tabular}{l|l|l|}
27658 & WVE15884 \\
\end{tabular} & 7/11/08 10:32 & 436.567 & 9 \\
\hline WVE15884-10 & \begin{tabular}{|l|l|}
27900 & WVE15884 \\
\end{tabular} & 7/14/08 13:39 & 436.5816 & 10 \\
\hline WVE15884-11 & $28219 \mid$ WVE15884 & 7/18/08 14:29 & 436.6076 & 11 \\
\hline WVE15884-12 & \begin{tabular}{|l|l|}
28836 & WVE15884 \\
\end{tabular} & $7 / 25 / 08$ 16:09 & 436.6107 & 12 \\
\hline WVE15884-13 & \begin{tabular}{l|l|l|}
29222 & WVE15884 \\
\end{tabular} & 7/30/08 12:01 & 436.6163 & 13 \\
\hline WVE15884-14 & \begin{tabular}{|l|l|}
29620 & WVE15884 \\
\end{tabular} & 8/7/08 19:03 & 436.6296 & 14 \\
\hline WVE15885-1 & 26713|WVE15885 & 7/2/08 14:35 & 432.241 & 1 \\
\hline WVE15885-2 & \begin{tabular}{l|l|l}
26820 & WVE15885 \\
\end{tabular} & 7/3/08 10:28 & 432.2381 & 2 \\
\hline WVE15885-3 & $26919 \mid$ WVE15885 & 7/3/08 15:06 & 432.2335 & 3 \\
\hline WVE15885-4 & \begin{tabular}{l|l|l}
27030 & WVE15885 \\
\end{tabular} & 7/7/08 10:00 & 432.2288 & 4 \\
\hline WVE15885-5 & \begin{tabular}{l|l|l|}
27231 & WVE15885 \\
\end{tabular} & 7/8/08 15:02 & 432.0762 & 5 \\
\hline WVE15885-6 & 27312 WVE15885 & 7/8/08 21:56 & 432.0732 & 6 \\
\hline WVE15885-7 & \begin{tabular}{l|l|}
27421 & WVE15885 \\
\end{tabular} & 7/9/08 10:43 & 432.09 & 7 \\
\hline WVE15885-8 & \begin{tabular}{l|l|l}
27550 & WVE15885
\end{tabular} & 7/10/08 16:12 & 432.0954 & 8 \\
\hline WVE15885-9 & 27659 WVE15885 & 7/11/08 10:34 & 432.1084 & 9 \\
\hline WVE15885-10 & \begin{tabular}{|l|l|}
27901 & WVE15885 \\
\end{tabular} & 7/14/08 13:41 & 432.1182 & 10 \\
\hline WVE15885-11 & 28220 $\mid$ WVE15885 & 7/18/08 14:31 & 432.1289 & 11 \\
\hline WVE15885-12 & 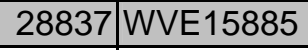 & 7/25/08 16:10 & 432.1393 & 12 \\
\hline WVE15885-13 & 29223|WVE15885 & $7 / 30 / 08$ 12:02 & 432.1541 & 13 \\
\hline WVE15885-14 & \begin{tabular}{|l|l|}
29621 & WVE15885 \\
\end{tabular} & 8/7/08 19:04 & 432.1647 & 14 \\
\hline WVE15886-1 & 26714 WVE15886 & $7 / 2 / 08$ 14:36 & 433.5625 & 1 \\
\hline WVE15886-2 & \begin{tabular}{|l|l|}
26821 & WVE15886 \\
\end{tabular} & $7 / 3 / 08$ 10:29 & 433.553 & 2 \\
\hline WVE15886-3 & \begin{tabular}{|l|l|}
26928 & WVE15886 \\
\end{tabular} & 7/3/08 15:14 & 433.5524 & 3 \\
\hline WVE15886-4 & \begin{tabular}{l|l|}
27031 & WVE15886 \\
\end{tabular} & 7/7/08 10:01 & 433.5516 & 4 \\
\hline WVE15886-5 & 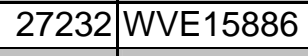 & 7/8/08 15:02 & 431.6898 & 5 \\
\hline WVE15886-6 & 27303 WVE15886 & 7/8/08 21:45 & 431.6827 & 6 \\
\hline WVE15886-7 & 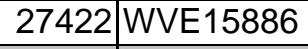 & 7/9/08 10:44 & 431.6896 & 7 \\
\hline WVE15886-8 & 27551 WVE15886 & 7/10/08 16:12 & 431.6911 & 8 \\
\hline WVE15886-9 & \begin{tabular}{l|l|l|}
27660 & WVE15886 \\
\end{tabular} & 7/11/08 10:35 & 431.6903 & 9 \\
\hline WVE15886-10 & \begin{tabular}{|l|l|}
27902 & WVE15886 \\
\end{tabular} & 7/14/08 13:42 & 431.6931 & 10 \\
\hline WVE15886-11 & \begin{tabular}{|l|l|}
28221 & WVE15886 \\
\end{tabular} & 7/18/08 14:32 & 431.6979 & 11 \\
\hline WVE15886-12 & 28838 WVE15886 & 7/25/08 16:11 & 431.7009 & 12 \\
\hline WVE15886-13 & 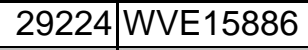 & 7/30/08 12:03 & 431.7057 & 13 \\
\hline WVE15886-14 & 29622 WVE15886 & 8/7/08 19:05 & 431.7137 & 14 \\
\hline WVE15887-1 & \begin{tabular}{l|l|l}
26715 & WVE15887 \\
\end{tabular} & $7 / 2 / 08$ 14:38 & 433.9369 & 1 \\
\hline WVE15887-2 & \begin{tabular}{l|l|l}
26822 & WVE15887 \\
\end{tabular} & $7 / 3 / 08$ 10:30 & 433.9303 & 2 \\
\hline WVE15887-3 & 26927|WVE15887 & 7/3/08 15:13 & 433.9279 & 3 \\
\hline
\end{tabular}




\begin{tabular}{|c|c|c|c|c|}
\hline WVE15887-4 & \begin{tabular}{l|l|l|}
27032 & WVE15887 \\
\end{tabular} & 7/7/08 10:02 & 433.9254 & 4 \\
\hline WVE15887-5 & \begin{tabular}{l|l|} 
27233 & WVE15887 \\
\end{tabular} & $7 / 8 / 08$ 15:03 & 432.9231 & 5 \\
\hline WVE15887-6 & \begin{tabular}{l|l}
27304 & WVE15887
\end{tabular} & $7 / 8 / 0821: 47$ & 432.918 & 6 \\
\hline WVE15887-7 & \begin{tabular}{l|l}
27423 & WVE15887 \\
\end{tabular} & $7 / 9 / 08$ 10:45 & 432.9191 & 7 \\
\hline WVE15887-8 & \begin{tabular}{l|l}
27552 & WVE15887
\end{tabular} & 7/10/08 16:13 & 432.9213 & 8 \\
\hline WVE15887-9 & 27661 $\mid$ WVE15887 & 7/11/08 10:35 & 432.9192 & 9 \\
\hline WVE15887-10 & 27903 WVE15887 & 7/14/08 13:43 & 432.9217 & 10 \\
\hline WVE15887-11 & \begin{tabular}{l|l} 
28222 & WVE15887
\end{tabular} & $7 / 18 / 08$ 14:34 & 432.9238 & 11 \\
\hline WVE15887-12 & 28839 WVE15887 & 7/25/08 16:12 & 432.928 & 12 \\
\hline WVE15887-13 & \begin{tabular}{l|l}
29225 & WVE15887
\end{tabular} & 7/30/08 12:03 & 432.9375 & 13 \\
\hline WVE15887-14 & \begin{tabular}{l|l|}
29623 & WVE15887 \\
\end{tabular} & 8/7/08 19:06 & 432.9444 & 14 \\
\hline WVE15888-1 & \begin{tabular}{l|l|}
26716 & WVE15888 \\
\end{tabular} & 7/2/08 14:39 & 434.9739 & 1 \\
\hline WVE15888-2 & \begin{tabular}{l|l|l|}
26823 & WVE15888
\end{tabular} & $7 / 3 / 08$ 10:31 & 434.9644 & 2 \\
\hline WVE15888-3 & \begin{tabular}{l|l|l|}
26926 & WVE15888
\end{tabular} & $7 / 3 / 08$ 15:13 & 434.9641 & 3 \\
\hline WVE15888-4 & $27033 \mid$ WVE15888 & 7/7/08 10:03 & 434.9625 & 4 \\
\hline WVE15888-5 & 27234 $\mid$ WVE15888 & $7 / 8 / 08$ 15:04 & 433.2275 & 5 \\
\hline WVE15888-6 & \begin{tabular}{l|l|l}
27305 & WVE15888 \\
\end{tabular} & $7 / 8 / 0821: 47$ & 433.2247 & 6 \\
\hline WVE15888-7 & 27424|WVE15888 & $7 / 9 / 08$ 10:46 & 433.2288 & 7 \\
\hline WVE15888-8 & \begin{tabular}{l|l|l}
27553 & WVE15888 \\
\end{tabular} & $7 / 10 / 08$ 16:15 & 433.2293 & 8 \\
\hline WVE15888-9 & \begin{tabular}{l|l|}
27662 & WVE15888 \\
\end{tabular} & 7/11/08 10:36 & 433.225 & 9 \\
\hline WVE15888-10 & 27904|WVE15888 & $7 / 14 / 08$ 13:44 & 433.2297 & 10 \\
\hline WVE15888-11 & \begin{tabular}{|l|l|}
28223 & WVE15888 \\
\end{tabular} & $7 / 18 / 08$ 14:35 & 433.2353 & 11 \\
\hline WVE15888-12 & \begin{tabular}{l|l|l}
28840 & WVE15888
\end{tabular} & 7/25/08 16:13 & 433.2368 & 12 \\
\hline WVE15888-13 & \begin{tabular}{|l|l|}
29226 & WVE15888 \\
\end{tabular} & 7/30/08 12:04 & 433.2454 & 13 \\
\hline WVE15888-14 & 29624|WVE15888 & 8/7/08 19:07 & 433.2504 & 14 \\
\hline WVE15889-1 & \begin{tabular}{l|l|l|}
26717 & WVE15889 \\
\end{tabular} & $7 / 2 / 08$ 14:40 & 437.6277 & 1 \\
\hline WVE15889-2 & 26824|WVE15889 & 7/3/08 10:32 & 437.6218 & 2 \\
\hline WVE15889-3 & 26925|WVE15889 & 7/3/08 15:11 & 437.6177 & 3 \\
\hline WVE15889-4 & \begin{tabular}{l|l|}
27034 & WVE15889
\end{tabular} & 7/7/08 10:04 & 437.6157 & 4 \\
\hline WVE15889-5 & \begin{tabular}{l|l|l|}
27235 & WVE15889
\end{tabular} & $7 / 8 / 08$ 15:05 & 436.2116 & 5 \\
\hline WVE15889-6 & \begin{tabular}{l|l|l}
27306 & WVE15889 \\
\end{tabular} & $7 / 8 / 0821: 48$ & 436.2063 & 6 \\
\hline WVE15889-7 & \begin{tabular}{l|l|l|}
27425 & WVE15889
\end{tabular} & 7/9/08 10:47 & 436.206 & 7 \\
\hline WVE15889-8 & \begin{tabular}{l|l|l|}
27554 & WVE15889 \\
\end{tabular} & 7/10/08 16:16 & 436.2067 & 8 \\
\hline WVE15889-9 & \begin{tabular}{l|l|}
27663 & WVE15889 \\
\end{tabular} & $7 / 11 / 08$ 10:37 & 436.2027 & 9 \\
\hline WVE15889-10 & \begin{tabular}{l|l|l|}
27905 & WVE15889
\end{tabular} & $7 / 14 / 08$ 13:45 & 436.2051 & 10 \\
\hline WVE15889-11 & 28224|WVE15889 & $7 / 18 / 08$ 14:36 & 436.2057 & 11 \\
\hline WVE15889-12 & \begin{tabular}{l|l|}
28841 & WVE15889 \\
\end{tabular} & 7/25/08 16:14 & 436.2139 & 12 \\
\hline WVE15889-13 & 29227 $\mid$ WVE15889 & $7 / 30 / 08$ 12:05 & 436.2196 & 13 \\
\hline WVE15889-14 & $29625 \mid$ WVE15889 & 8/7/08 19:08 & 436.2228 & 14 \\
\hline WVE15890-1 & \begin{tabular}{l|l|}
26718 & WVE15890 \\
\end{tabular} & 7/2/08 14:41 & 436.9581 & 1 \\
\hline WVE15890-2 & $26825 \mid$ WVE15890 & $7 / 3 / 08$ 10:32 & 436.9521 & 2 \\
\hline WVE15890-3 & 26924 $\mid$ WVE15890 & $7 / 3 / 08$ 15:11 & 436.9478 & 3 \\
\hline WVE15890-4 & 27035 WVE15890 & 7/7/08 10:04 & 436.9465 & 4 \\
\hline WVE15890-5 & \begin{tabular}{l|l}
27236 & WVE15890
\end{tabular} & 7/8/08 15:06 & 435.5688 & 5 \\
\hline WVE15890-6 & 27307 $\mid$ WVE15890 & $7 / 8 / 0821: 50$ & 435.562 & 6 \\
\hline WVE15890-7 & \begin{tabular}{l|l}
27426 & WVE15890
\end{tabular} & $7 / 9 / 08$ 10:49 & 435.5645 & 7 \\
\hline WVE15890-8 & $27555 \mid$ WVE15890 & 7/10/08 16:17 & 435.5651 & 8 \\
\hline WVE15890-9 & \begin{tabular}{l|l|l|}
27664 & WVE15890 \\
\end{tabular} & 7/11/08 10:39 & 435.5643 & 9 \\
\hline WVE15890-10 & \begin{tabular}{l|l|}
27906 & WVE15890 \\
\end{tabular} & $7 / 14 / 08$ 13:46 & 435.5683 & 10 \\
\hline
\end{tabular}




\begin{tabular}{|c|c|c|c|c|}
\hline WVE15890-11 & $28225 \mid$ WVE15890 & 7/18/08 14:37 & 435.5703 & 11 \\
\hline WVE15890-12 & 28842 |WVE15890 & 7/25/08 16:15 & 435.5785 & 12 \\
\hline WVE15890-13 & \begin{tabular}{|l|l|}
29228 & WVE15890
\end{tabular} & 7/30/08 12:06 & 435.587 & 13 \\
\hline WVE15890-14 & \begin{tabular}{l|l|}
29626 & WVE15890
\end{tabular} & 8/7/08 19:09 & 435.5897 & 14 \\
\hline WVE15891-1 & \begin{tabular}{l|l|l|}
26722 & WVE15891 \\
\end{tabular} & 7/2/08 14:48 & 429.9524 & 1 \\
\hline WVE15891-2 & \begin{tabular}{l|l|}
26828 & WVE15891 \\
\end{tabular} & 7/3/08 10:36 & 429.9458 & 2 \\
\hline WVE15891-3 & \begin{tabular}{l|l|l|}
26931 & WVE15891 \\
\end{tabular} & 7/3/08 15:17 & 429.9407 & 3 \\
\hline WVE15891-4 & \begin{tabular}{l|l|l}
27037 & WVE15891 \\
\end{tabular} & 7/7/08 10:08 & 429.9408 & 4 \\
\hline WVE15891-5 & 27084|WVE15891 & 7/7/08 17:54 & 430.1475 & 5 \\
\hline WVE15891-6 & \begin{tabular}{l|l|}
27170 & WVE15891 \\
\end{tabular} & $7 / 8 / 08$ 10:06 & 430.1501 & 6 \\
\hline WVE15891-7 & \begin{tabular}{l|l|l|}
27429 & WVE15891 \\
\end{tabular} & 7/9/08 10:51 & 430.153 & 7 \\
\hline WVE15891-8 & \begin{tabular}{|l|l|}
27563 & WVE15891
\end{tabular} & 7/10/08 16:26 & 430.1505 & 8 \\
\hline WVE15891-9 & \begin{tabular}{l|l|l|}
27667 & WVE15891 \\
\end{tabular} & 7/11/08 10:41 & 430.1513 & 9 \\
\hline WVE15891-10 & 27909 WVE15891 & 7/14/08 13:50 & 430.1531 & 10 \\
\hline WVE15891-11 & \begin{tabular}{|l|l|}
28228 & WVE15891 \\
\end{tabular} & 7/18/08 14:42 & 430.156 & 11 \\
\hline WVE15891-12 & \begin{tabular}{|l|l|}
28850 & WVE15891 \\
\end{tabular} & $7 / 25 / 08$ 16:24 & 430.1616 & 12 \\
\hline WVE15891-13 & 29231 $\mid$ WVE15891 & $7 / 30 / 08$ 12:09 & 430.1678 & 13 \\
\hline WVE15891-14 & 29629 WVE15891 & 8/7/08 19:11 & 430.1696 & 14 \\
\hline WVE15892-1 & 26723|WVE15892 & $7 / 2 / 08$ 14:49 & 429.9182 & 1 \\
\hline WVE15892-2 & 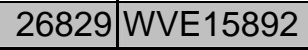 & $7 / 3 / 08$ 10:37 & 429.9122 & 2 \\
\hline WVE15892-3 & \begin{tabular}{l|l|l|}
26932 & WVE15892 \\
\end{tabular} & 7/3/08 15:18 & 429.9085 & 3 \\
\hline WVE15892-4 & 27038 WVE15892 & 7/7/08 10:09 & 429.9082 & 4 \\
\hline WVE15892-5 & $27085 \mid$ WVE15892 & 7/7/08 17:55 & 430.1844 & 5 \\
\hline WVE15892-6 & $27171 \mid$ WVE15892 & 7/8/08 10:06 & 430.1892 & 6 \\
\hline WVE15892-7 & 27430 $\mid$ WVE15892 & 7/9/08 10:52 & 430.1899 & 7 \\
\hline WVE15892-8 & \begin{tabular}{l|l|l}
27564 & WVE15892
\end{tabular} & 7/10/08 16:27 & 430.1865 & 8 \\
\hline WVE15892-9 & 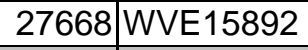 & 7/11/08 10:42 & 430.189 & 9 \\
\hline WVE15892-10 & \begin{tabular}{l|l|l}
27910 & WVE15892 \\
\end{tabular} & 7/14/08 13:51 & 430.1917 & 10 \\
\hline WVE15892-11 & 28229 WVE15892 & 7/18/08 14:44 & 430.1934 & 11 \\
\hline WVE15892-12 & \begin{tabular}{l|l|l}
28851 & WVE15892 \\
\end{tabular} & $7 / 25 / 08$ 16:25 & 430.1945 & 12 \\
\hline WVE15892-13 & \begin{tabular}{l|l|l|}
29232 & WVE15892 \\
\end{tabular} & $7 / 30 / 08$ 12:09 & 430.1976 & 13 \\
\hline WVE15892-14 & 29630 WVE15892 & 8/7/08 19:12 & 430.1968 & 14 \\
\hline WVE15893-1 & $26724 \mid$ WVE15893 & $7 / 2 / 08$ 14:50 & 427.8988 & 1 \\
\hline WVE15893-2 & \begin{tabular}{|l|l|}
26830 & WVE15893 \\
\end{tabular} & 7/3/08 10:38 & 427.8928 & 2 \\
\hline WVE15893-3 & 26933 WVE15893 & 7/3/08 15:19 & 427.8883 & 3 \\
\hline WVE15893-4 & 27039 WVE15893 & $7 / 7 / 08$ 10:10 & 427.8919 & 4 \\
\hline WVE15893-5 & 27086 WVE15893 & 7/7/08 17:56 & 428.0319 & 5 \\
\hline WVE15893-6 & 27172 WVE15893 & 7/8/08 10:07 & 428.0356 & 6 \\
\hline WVE15893-7 & 27431 $\mid$ WVE15893 & 7/9/08 10:53 & 428.035 & 7 \\
\hline WVE15893-8 & 27565 WVE15893 & 7/10/08 16:28 & 428.0365 & 8 \\
\hline WVE15893-9 & 27669 WVE15893 & 7/11/08 10:43 & 428.0351 & 9 \\
\hline WVE15893-10 & \begin{tabular}{|l|l|}
27911 & WVE15893 \\
\end{tabular} & 7/14/08 13:52 & 428.0396 & 10 \\
\hline WVE15893-11 & $28230 \mid$ WVE15893 & $7 / 18 / 08$ 14:45 & 428.0394 & 11 \\
\hline WVE15893-12 & \begin{tabular}{|l|l|}
28852 & WVE15893 \\
\end{tabular} & 7/25/08 16:27 & 428.0404 & 12 \\
\hline WVE15893-13 & $29233 \mid$ WVE15893 & 7/30/08 12:10 & 428.049 & 13 \\
\hline WVE15893-14 & 29631 WVE15893 & 8/7/08 19:13 & 428.0534 & 14 \\
\hline WVE15894-1 & \begin{tabular}{l|l|l|}
26725 & WVE15894 \\
\end{tabular} & 7/2/08 14:51 & 422.4391 & 1 \\
\hline WVE15894-2 & \begin{tabular}{|l|l|}
26831 & WVE15894 \\
\end{tabular} & 7/3/08 10:39 & 422.4375 & 2 \\
\hline WVE15894-3 & 26934|WVE15894 & 7/3/08 15:20 & 422.4341 & 3 \\
\hline
\end{tabular}




\begin{tabular}{|c|c|c|c|c|}
\hline WVE15894-4 & 27040|WVE15894 & 7/7/08 10:11 & 422.4351 & 4 \\
\hline WVE15894-5 & \begin{tabular}{l|l|}
27087 & WVE15894
\end{tabular} & 7/7/08 17:56 & 422.6802 & 5 \\
\hline WVE15894-6 & \begin{tabular}{l|l|l|}
27173 & WVE15894
\end{tabular} & 7/8/08 10:08 & 422.6813 & 6 \\
\hline WVE15894-7 & \begin{tabular}{l|l|}
27432 & WVE15894 \\
\end{tabular} & 7/9/08 10:54 & 422.6865 & 7 \\
\hline WVE15894-8 & \begin{tabular}{|l|l|}
27566 & WVE15894 \\
\end{tabular} & $7 / 10 / 08$ 16:29 & 422.6824 & 8 \\
\hline WVE15894-9 & \begin{tabular}{l|l|l|}
27670 & WVE15894 \\
\end{tabular} & 7/11/08 10:43 & 422.6813 & 9 \\
\hline WVE15894-10 & \begin{tabular}{|l|l|}
27912 & WVE15894 \\
\end{tabular} & 7/14/08 13:53 & 422.6847 & 10 \\
\hline WVE15894-11 & \begin{tabular}{l|l|l|}
28231 & WVE15894 \\
\end{tabular} & $7 / 18 / 08$ 14:46 & 422.6864 & 11 \\
\hline WVE15894-12 & \begin{tabular}{l|l|l|}
28853 & WVE15894 \\
\end{tabular} & $7 / 25 / 08$ 16:28 & 422.6893 & 12 \\
\hline WVE15894-13 & \begin{tabular}{l|l|}
29234 & WVE15894 \\
\end{tabular} & $7 / 30 / 08$ 12:11 & 422.6966 & 13 \\
\hline WVE15894-14 & \begin{tabular}{l|l|}
29632 & WVE15894
\end{tabular} & 8/7/08 19:14 & 422.6996 & 14 \\
\hline WVE15895-1 & \begin{tabular}{l|l|l|}
26726 & WVE15895 \\
\end{tabular} & $7 / 2 / 08$ 14:52 & 425.6377 & 1 \\
\hline WVE15895-2 & \begin{tabular}{l|l|l} 
26832 & WVE15895
\end{tabular} & $7 / 3 / 08$ 10:40 & 425.6466 & 2 \\
\hline WVE15895-3 & 26935|WVE15895 & $7 / 3 / 08$ 15:21 & 425.635 & 3 \\
\hline WVE15895-4 & \begin{tabular}{l|l|l|}
27041 & WVE15895 \\
\end{tabular} & 7/7/08 10:12 & 425.6358 & 4 \\
\hline WVE15895-5 & \begin{tabular}{|l|l|}
27088 & WVE15895
\end{tabular} & 7/7/08 17:57 & 425.7695 & 5 \\
\hline WVE15895-6 & 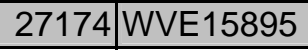 & 7/8/08 10:09 & 425.7696 & 6 \\
\hline WVE15895-7 & 27433 WVE15895 & 7/9/08 10:55 & 425.7768 & 7 \\
\hline WVE15895-8 & \begin{tabular}{|l|l|}
27567 & WVE15895 \\
\end{tabular} & 7/10/08 16:30 & 425.7701 & 8 \\
\hline WVE15895-9 & \begin{tabular}{l|l|l|}
27671 & WVE15895 \\
\end{tabular} & 7/11/08 10:44 & 425.7702 & 9 \\
\hline WVE15895-10 & 27913 WVE15895 & 7/14/08 13:54 & 425.7734 & 10 \\
\hline WVE15895-11 & \begin{tabular}{l|l|l}
28232 & WVE15895 \\
\end{tabular} & 7/18/08 14:48 & 425.7723 & 11 \\
\hline WVE15895-12 & \begin{tabular}{l|l|l|}
28854 & WVE15895 \\
\end{tabular} & $7 / 25 / 08$ 16:29 & 425.7828 & 12 \\
\hline WVE15895-13 & $29235 \mid$ WVE15895 & 7/30/08 12:12 & 425.7934 & 13 \\
\hline WVE15895-14 & 29633 WVE15895 & 8/7/08 19:15 & 425.7942 & 14 \\
\hline WVE15896-1 & \begin{tabular}{l|l|l}
26721 & WVE15896 \\
\end{tabular} & $7 / 2 / 08$ 14:45 & 433.0251 & 1 \\
\hline WVE15896-2 & 26833|WVE15896 & 7/3/08 10:41 & 433.0203 & 2 \\
\hline WVE15896-3 & \begin{tabular}{|l|l|}
26936 & WVE15896 \\
\end{tabular} & $7 / 3 / 08$ 15:22 & 433.0165 & 3 \\
\hline WVE15896-4 & \begin{tabular}{l|l|l|}
27042 & WVE15896 \\
\end{tabular} & 7/7/08 10:13 & 433.0169 & 4 \\
\hline WVE15896-5 & 27091 $\mid$ WVE15896 & 7/7/08 17:59 & 433.1268 & 5 \\
\hline WVE15896-6 & 27177 WVE15896 & 7/8/08 10:13 & 433.1301 & 6 \\
\hline WVE15896-7 & $27434 \mid$ WVE15896 & 7/9/08 10:55 & 433.1304 & 7 \\
\hline WVE15896-8 & \begin{tabular}{l|l|l|}
27558 & WVE15896 \\
\end{tabular} & 7/10/08 16:21 & 433.1291 & 8 \\
\hline WVE15896-9 & 27672 WVE15896 & 7/11/08 10:45 & 433.1278 & 9 \\
\hline WVE15896-10 & 27914 WVE15896 & 7/14/08 13:55 & 433.1326 & 10 \\
\hline WVE15896-11 & 28233 WVE15896 & $7 / 18 / 08$ 14:49 & 433.1341 & 11 \\
\hline WVE15896-12 & 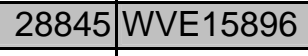 & $7 / 25 / 08$ 16:19 & 433.1393 & 12 \\
\hline WVE15896-13 & 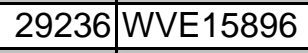 & $7 / 30 / 08$ 12:13 & 433.1481 & 13 \\
\hline WVE15896-14 & 29634 WVE15896 & 8/7/08 19:16 & 433.1508 & 14 \\
\hline WVE15897-1 & \begin{tabular}{l|l|}
26727 & WVE15897 \\
\end{tabular} & $7 / 2 / 08$ 14:52 & 433.588 & 1 \\
\hline WVE15897-2 & \begin{tabular}{l|l|}
26834 & WVE15897 \\
\end{tabular} & $7 / 3 / 08$ 10:42 & 433.5804 & 2 \\
\hline WVE15897-3 & \begin{tabular}{l|l|l|}
26937 & WVE15897 \\
\end{tabular} & $7 / 3 / 08$ 15:23 & 433.5787 & 3 \\
\hline WVE15897-4 & 27043 WVE15897 & 7/7/08 10:14 & 433.582 & 4 \\
\hline WVE15897-5 & 27092 WVE15897 & 7/7/08 18:00 & 433.7459 & 5 \\
\hline WVE15897-6 & \begin{tabular}{l|l|l|}
27178 & WVE15897 \\
\end{tabular} & 7/8/08 10:14 & 433.7486 & 6 \\
\hline WVE15897-7 & 27435 WVE15897 & $7 / 9 / 08$ 10:56 & 433.7477 & 7 \\
\hline WVE15897-8 & 27559 WVE15897 & 7/10/08 16:22 & 433.7482 & 8 \\
\hline WVE15897-9 & 27673 WVE15897 & 7/11/08 10:46 & 433.7458 & 9 \\
\hline WVE15897-10 & \begin{tabular}{l|l|l|}
27915 & WVE15897 \\
\end{tabular} & 7/14/08 13:56 & 433.7504 & 10 \\
\hline
\end{tabular}




\begin{tabular}{|c|c|c|c|c|}
\hline WVE15897-11 & \begin{tabular}{l|l|} 
28234 & WVE15897
\end{tabular} & 7/18/08 14:50| & 433.7474 & 11 \\
\hline WVE15897-12 & \begin{tabular}{l|l|}
28846 & WVE15897 \\
\end{tabular} & $7 / 25 / 08$ 16:20 & 433.7498 & 12 \\
\hline WVE15897-13 & 29237|WVE15897 & 7/30/08 12:13 & 433.7593 & 13 \\
\hline WVE15897-14 & \begin{tabular}{l|l|}
29635 & WVE15897
\end{tabular} & 8/7/08 19:17 & 433.7594 & 14 \\
\hline WVE15898-1 & 26728|WVE15898 & $7 / 2 / 08$ 14:53 & 435.3694 & 1 \\
\hline WVE15898-2 & 26835|WVE15898 & 7/3/08 10:43 & 435.3665 & 2 \\
\hline WVE15898-3 & 26938|WVE15898 & $7 / 3 / 08$ 15:24 & 435.365 & 3 \\
\hline WVE15898-4 & \begin{tabular}{l|l|}
27044 & WVE15898
\end{tabular} & 7/7/08 10:14 & 435.3653 & 4 \\
\hline WVE15898-5 & \begin{tabular}{|l|l|}
27093 & WVE15898 \\
\end{tabular} & 7/7/08 18:00 & 435.7586 & 5 \\
\hline WVE15898-6 & \begin{tabular}{|l|l|}
27179 & WVE15898
\end{tabular} & $7 / 8 / 08$ 10:15 & 435.7599 & 6 \\
\hline WVE15898-7 & 27436|WVE15898 & 7/9/08 10:57 & 435.7605 & 7 \\
\hline WVE15898-8 & 27560 $\mid$ WVE15898 & $7 / 10 / 08$ 16:23 & 435.7567 & 8 \\
\hline WVE15898-9 & 27674|WVE15898 & 7/11/08 10:47 & 435.7581 & 9 \\
\hline WVE15898-10 & \begin{tabular}{l|l|l|}
27916 & WVE15898 \\
\end{tabular} & 7/14/08 13:57| & 435.7623 & 10 \\
\hline WVE15898-11 & 28235|WVE15898 & $7 / 18 / 08$ 14:51 & 435.7615 & 11 \\
\hline WVE15898-12 & \begin{tabular}{l|l|}
28847 & WVE15898 \\
\end{tabular} & $7 / 25 / 08$ 16:21 & 435.7624 & 12 \\
\hline WVE15898-13 & 29238|WVE15898 & $7 / 30 / 08$ 12:14 & 435.7705 & 13 \\
\hline WVE15898-14 & \begin{tabular}{|l|l|} 
29636 & WVE15898 \\
\end{tabular} & 8/7/08 19:17 & 435.774 & 14 \\
\hline WVE15899-1 & $26729 \mid$ WVE15899 & $7 / 2 / 08$ 14:54 & 436.1967 & 1 \\
\hline WVE15899-2 & \begin{tabular}{|l|l|}
26836 & WVE15899 \\
\end{tabular} & $7 / 3 / 08$ 10:44 & 436.1975 & 2 \\
\hline WVE15899-3 & 26939|WVE15899 & 7/3/08 15:25 & 436.1941 & 3 \\
\hline WVE15899-4 & \begin{tabular}{l|l|l|}
27045 & WVE15899 \\
\end{tabular} & $7 / 7 / 08$ 10:15 & 436.1909 & 4 \\
\hline WVE15899-5 & 27094|WVE15899 & 7/7/08 18:01 & 436.3663 & 5 \\
\hline WVE15899-6 & 27180 $\mid$ WVE15899 & 7/8/08 10:16 & 436.3694 & 6 \\
\hline WVE15899-7 & 27437 $\mid$ WVE15899 & 7/9/08 10:58 & 436.3691 & 7 \\
\hline WVE15899-8 & 27561 $\mid$ WVE15899 & $7 / 10 / 08$ 16:24 & 436.3667 & 8 \\
\hline WVE15899-9 & 27675|WVE15899 & 7/11/08 10:47 & 436.3695 & 9 \\
\hline WVE15899-10 & \begin{tabular}{l|l|l|}
27917 & WVE15899
\end{tabular} & 7/14/08 13:58 & 436.3698 & 10 \\
\hline WVE15899-11 & 28236|WVE15899 & $7 / 18 / 08$ 14:52 & 436.3707 & 11 \\
\hline WVE15899-12 & 28848|WVE15899 & $7 / 25 / 08$ 16:22 & 436.3715 & 12 \\
\hline WVE15899-13 & 29239 WVE15899 & 7/30/08 12:15 & 436.3753 & 13 \\
\hline WVE15899-14 & 29637 $\mid$ WVE15899 & 8/7/08 19:18 & 436.3786 & 14 \\
\hline WVE15900-1 & 26730|WVE15900 & $7 / 2 / 08$ 14:55 & 431.6795 & 1 \\
\hline WVE15900-2 & \begin{tabular}{l|l|l|}
26837 & WVE15900
\end{tabular} & 7/3/08 10:45 & 431.6759 & 2 \\
\hline WVE15900-3 & \begin{tabular}{|l|l|}
26940 & WVE15900 \\
\end{tabular} & $7 / 3 / 08$ 15:26 & 431.6784 & 3 \\
\hline WVE15900-4 & \begin{tabular}{l|l|}
27046 & WVE15900
\end{tabular} & 7/7/08 10:16 & 431.6719 & 4 \\
\hline WVE15900-5 & 27095|WVE15900 & 7/7/08 18:02 & 431.8095 & 5 \\
\hline WVE15900-6 & \begin{tabular}{l|l}
27181 & WVE15900
\end{tabular} & 7/8/08 10:16 & 431.815 & 6 \\
\hline WVE15900-7 & \begin{tabular}{|l|l|}
27438 & WVE15900 \\
\end{tabular} & 7/9/08 10:59 & 431.8191 & 7 \\
\hline WVE15900-8 & \begin{tabular}{l|l|l|}
27562 & WVE15900
\end{tabular} & $7 / 10 / 08$ 16:25 & 431.8145 & 8 \\
\hline WVE15900-9 & 27676|WVE15900 & 7/11/08 10:48 & 431.8148 & 9 \\
\hline WVE15900-10 & 27918 $\mid$ WVE15900 & 7/14/08 13:59 & 431.8164 & 10 \\
\hline WVE15900-11 & 28237 $\mid$ WVE15900 & $7 / 18 / 08$ 14:53 & 431.8186 & 11 \\
\hline WVE15900-12 & 28849 WVE15900 & $7 / 25 / 08$ 16:23 & 431.8224 & 12 \\
\hline WVE15900-13 & 29240|WVE15900 & $7 / 30 / 08$ 12:16 & 431.8277 & 13 \\
\hline WVE15900-14 & 29638|WVE15900 & 8/7/08 19:19 & 431.8333 & 14 \\
\hline WVE15901-1 & 26733|WVE15901 & $7 / 2 / 08$ 14:59 & 430.3674 & 1 \\
\hline WVE15901-2 & 26840 WVE15901 & 7/3/08 10:48 & 430.3576 & 2 \\
\hline WVE15901-3 & \begin{tabular}{l|l|}
26943 & WVE15901 \\
\end{tabular} & $7 / 3 / 08$ 15:28 & 430.3535 & 3 \\
\hline
\end{tabular}




\begin{tabular}{|c|c|c|c|c|}
\hline WVE15901-4 & 27049 WVE15901 & 7/7/08 10:21 & 430.3524 & 4 \\
\hline WVE15901-5 & 27109|WVE15901 & 7/7/08 19:50 & 429.8134 & 5 \\
\hline WVE15901-6 & \begin{tabular}{l|l|l}
27182 & WVE15901
\end{tabular} & $7 / 8 / 08$ 10:17 & 429.8767 & 6 \\
\hline WVE15901-7 & \begin{tabular}{l|l|}
27315 & WVE15901 \\
\end{tabular} & 7/8/08 22:02 & 429.8985 & 7 \\
\hline WVE15901-8 & \begin{tabular}{l|l|l}
27441 & WVE15901 \\
\end{tabular} & 7/9/08 11:02 & 429.9159 & 8 \\
\hline WVE15901-9 & 27570|WVE15901 & $7 / 10 / 08$ 16:34 & 429.9376 & 9 \\
\hline WVE15901-10 & \begin{tabular}{l|l|l}
27679 & WVE15901 \\
\end{tabular} & 7/11/08 10:51 & 429.949 & 10 \\
\hline WVE15901-11 & \begin{tabular}{l|l|}
27921 & WVE15901
\end{tabular} & $7 / 14 / 08$ 14:03 & 429.9854 & 11 \\
\hline WVE15901-12 & 28240|WVE15901 & 7/18/08 14:58 & 430.0144 & 12 \\
\hline WVE15901-13 & \begin{tabular}{l|l|}
28857 & WVE15901
\end{tabular} & $7 / 25 / 08$ 16:33 & 430.0525 & 13 \\
\hline WVE15901-14 & \begin{tabular}{l|l|l|}
29243 & WVE15901 \\
\end{tabular} & 7/30/08 12:18 & 430.0814 & 14 \\
\hline WVE15901-15 & \begin{tabular}{|l|l|}
29641 & WVE15901 \\
\end{tabular} & 8/7/08 19:21 & 430.1056 & 15 \\
\hline WVE15902-1 & \begin{tabular}{l|l|l|}
26734 & WVE15902 \\
\end{tabular} & $7 / 2 / 08$ 15:00 & 451.5706 & 1 \\
\hline WVE15902-2 & \begin{tabular}{l|l|l|}
26841 & WVE15902 \\
\end{tabular} & $7 / 3 / 08$ 10:49 & 451.5674 & 2 \\
\hline WVE15902-3 & \begin{tabular}{l|l|l|}
26944 & WVE15902 \\
\end{tabular} & 7/3/08 15:30 & 451.563 & 3 \\
\hline WVE15902-4 & \begin{tabular}{l|l|l|}
27050 & WVE15902 \\
\end{tabular} & 7/7/08 10:21 & 451.564 & 4 \\
\hline WVE15902-5 & \begin{tabular}{l|l|l|}
27108 & WVE15902
\end{tabular} & 7/7/08 19:49 & 450.9869 & 5 \\
\hline WVE15902-6 & 27183|WVE15902 & 7/8/08 10:18 & 451.0611 & 6 \\
\hline WVE15902-7 & \begin{tabular}{l|l|l} 
27316 & WVE15902 \\
\end{tabular} & $7 / 8 / 08$ 22:03 & 451.0852 & 7 \\
\hline WVE15902-8 & 27442 |WVE15902 & 7/9/08 11:03 & 451.1051 & 8 \\
\hline WVE15902-9 & \begin{tabular}{l|l|}
27571 & WVE15902 \\
\end{tabular} & 7/10/08 16:35 & 451.1273 & 9 \\
\hline WVE15902-10 & \begin{tabular}{l|l|}
27680 & WVE15902 \\
\end{tabular} & 7/11/08 10:52 & 451.1428 & 10 \\
\hline WVE15902-11 & \begin{tabular}{l|l}
27922 & WVE15902 \\
\end{tabular} & $7 / 14 / 08$ 14:04 & 451.1783 & 11 \\
\hline WVE15902-12 & \begin{tabular}{l|l|l|}
28241 & WVE15902 \\
\end{tabular} & $7 / 18 / 08$ 14:59 & 451.2094 & 12 \\
\hline WVE15902-13 & \begin{tabular}{l|l}
28858 & WVE15902
\end{tabular} & 7/25/08 16:34 & 451.2487 & 13 \\
\hline WVE15902-14 & 29244|WVE15902 & 7/30/08 12:19 & 451.2715 & 14 \\
\hline WVE15902-15 & \begin{tabular}{l|l|l|}
29642 & WVE15902 \\
\end{tabular} & 8/7/08 19:22 & 451.3057 & 15 \\
\hline WVE15903-1 & $26735 \mid$ WVE15903 & 7/2/08 15:01 & 435.7914 & 1 \\
\hline WVE15903-2 & \begin{tabular}{l|l|l|}
26842 & WVE15903
\end{tabular} & $7 / 3 / 08$ 10:50 & 435.7923 & 2 \\
\hline WVE15903-3 & $26945 \mid$ WVE15903 & 7/3/08 15:31 & 435.7891 & 3 \\
\hline WVE15903-4 & \begin{tabular}{l|l|l}
27051 & WVE15903
\end{tabular} & 7/7/08 10:22 & 435.7837 & 4 \\
\hline WVE15903-5 & 27098|WVE15903 & 7/7/08 19:41 & 435.2483 & 5 \\
\hline WVE15903-6 & 27184 WVE15903 & $7 / 8 / 08$ 10:19 & 435.3179 & 6 \\
\hline WVE15903-7 & \begin{tabular}{l|l|l|}
27317 & WVE15903
\end{tabular} & $7 / 8 / 0822: 04$ & 435.3419 & 7 \\
\hline WVE15903-8 & 27443 WVE15903 & 7/9/08 11:04 & 435.3578 & 8 \\
\hline WVE15903-9 & \begin{tabular}{l|l|}
27572 & WVE15903 \\
\end{tabular} & $7 / 10 / 08$ 16:36 & 435.3793 & 9 \\
\hline WVE15903-10 & \begin{tabular}{l|l|l|}
27681 & WVE15903 \\
\end{tabular} & 7/11/08 10:53 & 435.3895 & 10 \\
\hline WVE15903-11 & 27923|WVE15903 & $7 / 14 / 08$ 14:05 & 435.4328 & 11 \\
\hline WVE15903-12 & \begin{tabular}{|l|l|}
28242 & WVE15903 \\
\end{tabular} & 7/18/08 15:00 & 435.4603 & 12 \\
\hline WVE15903-13 & 28859 WVE15903 & $7 / 25 / 08$ 16:35 & 435.4938 & 13 \\
\hline WVE15903-14 & 29245 WVE15903 & $7 / 30 / 08$ 12:20 & 435.5192 & 14 \\
\hline WVE15903-15 & 29643 WVE15903 & 8/7/08 19:23 & 435.5427 & 15 \\
\hline WVE15904-1 & \begin{tabular}{l|l|l|}
26736 & WVE15904 \\
\end{tabular} & 7/2/08 15:02 & 426.3835 & 1 \\
\hline WVE15904-2 & $26843 \mid$ WVE15904 & 7/3/08 10:51 & 426.3797 & 2 \\
\hline WVE15904-3 & \begin{tabular}{l|l|}
26946 & WVE15904 \\
\end{tabular} & $7 / 3 / 08$ 15:31 & 426.3754 & 3 \\
\hline WVE15904-4 & \begin{tabular}{l|l|l|}
27052 & WVE15904 \\
\end{tabular} & 7/7/08 10:23 & 426.372 & 4 \\
\hline WVE15904-5 & \begin{tabular}{l|l|}
27099 & WVE15904 \\
\end{tabular} & 7/7/08 19:42 & 425.8458 & 5 \\
\hline WVE15904-6 & \begin{tabular}{|l|l|l|}
27185 & WVE15904 \\
\end{tabular} & 7/8/08 10:20 & 425.9126 & 6 \\
\hline WVE15904-7 & 27318 $\mid$ WVE15904 & $7 / 8 / 0822: 05$ & 425.9355 & 7 \\
\hline
\end{tabular}




\begin{tabular}{|c|c|c|c|c|}
\hline WVE15904-8 & 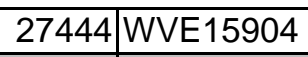 & 7/9/08 11:05 & 425.9529 & 8 \\
\hline WVE15904-9 & 27573|WVE15904 & 7/10/08 16:37| & 425.9707 & 9 \\
\hline WVE15904-10 & \begin{tabular}{|l|l|}
27682 & WVE15904 \\
\end{tabular} & 7/11/08 10:54 & 425.9838 & 10 \\
\hline WVE15904-11 & \begin{tabular}{l|l|l|}
27924 & WVE15904 \\
\end{tabular} & $7 / 14 / 08$ 14:06 & 426.0199 & 11 \\
\hline WVE15904-12 & \begin{tabular}{|l|l|}
28243 & WVE15904
\end{tabular} & 7/18/08 15:01 & 426.0521 & 12 \\
\hline WVE15904-13 & \begin{tabular}{l|l|l}
28860 & WVE15904 \\
\end{tabular} & 7/25/08 16:37 & 426.0816 & 13 \\
\hline WVE15904-14 & \begin{tabular}{l|l|l|}
29246 & WVE15904 \\
\end{tabular} & $7 / 30 / 08$ 12:21 & 426.1084 & 14 \\
\hline WVE15904-15 & \begin{tabular}{l|l|l|}
29644 & WVE15904 \\
\end{tabular} & 8/7/08 19:24 & 426.1336 & 15 \\
\hline WVE15905-1 & 26737|WVE15905 & 7/2/08 15:03 & 449.1079 & 1 \\
\hline WVE15905-2 & \begin{tabular}{l|l|l|}
26844 & WVE15905 \\
\end{tabular} & $7 / 3 / 08$ 10:52 & 449.1014 & 2 \\
\hline WVE15905-3 & \begin{tabular}{l|l|l}
26947 & WVE15905 \\
\end{tabular} & 7/3/08 15:33 & 449.0986 & 3 \\
\hline WVE15905-4 & 27053|WVE15905 & 7/7/08 10:24 & 449.0945 & 4 \\
\hline WVE15905-5 & $27100 \mid$ WVE15905 & 7/7/08 19:43 & 448.5409 & 5 \\
\hline WVE15905-6 & \begin{tabular}{|l|l|l|}
27186 & WVE15905 \\
\end{tabular} & 7/8/08 10:21 & 448.6107 & 6 \\
\hline WVE15905-7 & $27319 \mid$ WVE15905 & 7/8/08 22:06 & 448.6357 & 7 \\
\hline WVE15905-8 & \begin{tabular}{l|l|l|}
27445 & WVE15905 \\
\end{tabular} & 7/9/08 11:05 & 448.6488 & 8 \\
\hline WVE15905-9 & $27574 \mid$ WVE15905 & 7/10/08 16:38 & 448.6761 & 9 \\
\hline WVE15905-10 & 27683 WVE15905 & 7/11/08 10:55 & 448.6886 & 10 \\
\hline WVE15905-11 & $27925 \mid$ WVE15905 & $7 / 14 / 08$ 14:07 & 448.7224 & 11 \\
\hline WVE15905-12 & \begin{tabular}{l|l|l|}
28244 & WVE15905 \\
\end{tabular} & 7/18/08 15:02 & 448.7555 & 12 \\
\hline WVE15905-13 & \begin{tabular}{l|l|l|}
28861 & WVE15905 \\
\end{tabular} & $7 / 25 / 08$ 16:38 & 448.791 & 13 \\
\hline WVE15905-14 & \begin{tabular}{l|l|l|}
29247 & WVE15905 \\
\end{tabular} & $7 / 30 / 08$ 12:22 & 448.8213 & 14 \\
\hline WVE15905-15 & $29645 \mid$ WVE15905 & $8 / 7 / 08$ 19:25 & 448.8462 & 15 \\
\hline WVE15906-1 & 26738 WVE15906 & 7/2/08 15:04 & 430.8293 & 1 \\
\hline WVE15906-2 & \begin{tabular}{l|l|l|}
26845 & WVE15906 \\
\end{tabular} & 7/3/08 10:52 & 430.8256 & 2 \\
\hline WVE15906-3 & \begin{tabular}{l|l|l}
26952 & WVE15906
\end{tabular} & 7/3/08 15:38 & 430.8178 & 3 \\
\hline WVE15906-4 & $27054 \mid$ WVE15906 & 7/7/08 10:25 & 430.8193 & 4 \\
\hline WVE15906-5 & \begin{tabular}{|l|l|l|}
27101 & WVE15906 \\
\end{tabular} & 7/7/08 19:44 & 430.3031 & 5 \\
\hline WVE15906-6 & 27189 WVE15906 & 7/8/08 10:25 & 430.3697 & 6 \\
\hline WVE15906-7 & \begin{tabular}{|l|l|}
27320 & WVE15906 \\
\end{tabular} & 7/8/08 22:07 & 430.3908 & 7 \\
\hline WVE15906-8 & 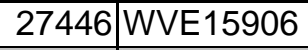 & 7/9/08 11:06 & 430.4062 & 8 \\
\hline WVE15906-9 & $27575 \mid$ WVE15906 & 7/10/08 16:39 & 430.4293 & 9 \\
\hline WVE15906-10 & \begin{tabular}{l|l|l|}
27684 & WVE15906 \\
\end{tabular} & 7/11/08 10:56 & 430.444 & 10 \\
\hline WVE15906-11 & \begin{tabular}{|l|l|}
27926 & WVE15906 \\
\end{tabular} & 7/14/08 14:08 & 430.4734 & 11 \\
\hline WVE15906-12 & $28245 \mid$ WVE15906 & 7/18/08 15:03 & 430.5008 & 12 \\
\hline WVE15906-13 & \begin{tabular}{l|l|}
28862 & WVE15906 \\
\end{tabular} & 7/25/08 16:39 & 430.5356 & 13 \\
\hline WVE15906-14 & 29248 $\mid$ WVE15906 & 7/30/08 12:22 & 430.5569 & 14 \\
\hline WVE15906-15 & \begin{tabular}{l|l|l|}
29646 & WVE15906 \\
\end{tabular} & 8/7/08 19:26 & 430.5793 & 15 \\
\hline WVE15907-1 & 26739 WVE15907 & 7/2/08 15:05 & 426.1481 & 1 \\
\hline WVE15907-2 & 26846 WVE15907 & 7/3/08 10:53 & 426.1419 & 2 \\
\hline WVE15907-3 & \begin{tabular}{|l|l|}
26948 & WVE15907 \\
\end{tabular} & 7/3/08 15:33 & 426.1388 & 3 \\
\hline WVE15907-4 & 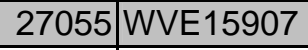 & 7/7/08 10:26 & 426.1363 & 4 \\
\hline WVE15907-5 & \begin{tabular}{l|l|l|}
27102 & WVE15907 \\
\end{tabular} & 7/7/08 19:45 & 425.6068 & 5 \\
\hline WVE15907-6 & 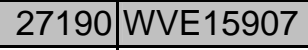 & 7/8/08 10:26 & 425.6753 & 6 \\
\hline WVE15907-7 & \begin{tabular}{l|l|l|}
27321 & WVE15907 \\
\end{tabular} & $7 / 8 / 08$ 22:09 & 425.6953 & 7 \\
\hline WVE15907-8 & 27447 WVE15907 & 7/9/08 11:07 & 425.7135 & 8 \\
\hline WVE15907-9 & \begin{tabular}{l|l|}
27576 & WVE15907 \\
\end{tabular} & 7/10/08 16:40 & 425.736 & 9 \\
\hline WVE15907-10 & 27685 WVE15907 & 7/11/08 10:57 & 425.7481 & 10 \\
\hline WVE15907-11 & 27927|WVE15907 & 7/14/08 14:09 & 425.7839 & 11 \\
\hline
\end{tabular}




\begin{tabular}{|c|c|c|c|c|}
\hline WVE15907-12 & 28246|WVE15907 & 7/18/08 15:04 & 425.8172 & 12 \\
\hline WVE15907-13 & 28863|WVE15907 & $7 / 25 / 08$ 16:41 & 425.8539 & 13 \\
\hline WVE15907-14 & 29249 WVE15907 & $7 / 30 / 08$ 12:23 & 425.8724 & 14 \\
\hline WVE15907-15 & \begin{tabular}{l|l|}
29647 & WVE15907
\end{tabular} & $8 / 7 / 08$ 19:26 & 425.897 & 15 \\
\hline WVE15908-1 & 26740|WVE15908 & 7/2/08 15:06 & 429.0839 & 1 \\
\hline WVE15908-2 & 26847 $\mid$ WVE15908 & $7 / 3 / 08$ 10:54 & 429.0782 & 2 \\
\hline WVE15908-3 & 26949|WVE15908 & $7 / 3 / 08$ 15:34 & 429.0747 & 3 \\
\hline WVE15908-4 & 27056 $\mid$ WVE15908 & 7/7/08 10:27| & 429.0664 & 4 \\
\hline WVE15908-5 & \begin{tabular}{|l|l|l|}
27103 & WVE15908 \\
\end{tabular} & $7 / 7 / 08$ 19:46 & 428.558 & 5 \\
\hline WVE15908-6 & 27191 $\mid$ WVE15908 & 7/8/08 10:27 & 428.6272 & 6 \\
\hline WVE15908-7 & \begin{tabular}{l|l|l|}
27322 & WVE15908 \\
\end{tabular} & $7 / 8 / 0822: 10$ & 428.6458 & 7 \\
\hline WVE15908-8 & 27448|WVE15908 & 7/9/08 11:08 & 428.667 & 8 \\
\hline WVE15908-9 & 27577 WVE15908 & $7 / 10 / 08$ 16:41 & 428.6885 & 9 \\
\hline WVE15908-10 & 27686|WVE15908 & 7/11/08 10:57 & 428.6979 & 10 \\
\hline WVE15908-11 & \begin{tabular}{|l|l|}
27928 & WVE15908 \\
\end{tabular} & 7/14/08 14:10 & 428.7361 & 11 \\
\hline WVE15908-12 & $\begin{array}{l}28247 \\
\text { WVE15908 }\end{array}$ & 7/18/08 15:05 & 428.7693 & 12 \\
\hline WVE15908-13 & 28864 WVE15908 & 7/25/08 16:42 & 428.8025 & 13 \\
\hline WVE15908-14 & 29250 WVE15908 & 7/30/08 12:24 & 428.8243 & 14 \\
\hline WVE15908-15 & \begin{tabular}{|l|l|}
29648 & WVE15908 \\
\end{tabular} & 8/7/08 19:27 & 428.8474 & 15 \\
\hline WVE15909-1 & 26741 $\mid$ WVE15909 & 7/2/08 15:07 & 444.884 & 1 \\
\hline WVE15909-2 & \begin{tabular}{|l|l|}
26848 & WVE15909 \\
\end{tabular} & $7 / 3 / 08$ 10:55 & 444.8773 & 2 \\
\hline WVE15909-3 & 26950 WVE15909 & $7 / 3 / 08$ 15:36 & 444.8648 & 3 \\
\hline WVE15909-4 & 27057 WVE15909 & 7/7/08 10:28 & 444.8638 & 4 \\
\hline WVE15909-5 & $27104 \mid$ WVE15909 & 7/7/08 19:47 & 444.2624 & 5 \\
\hline WVE15909-6 & \begin{tabular}{l|l|l|}
27192 & WVE15909
\end{tabular} & $7 / 8 / 08$ 10:28 & 444.3329 & 6 \\
\hline WVE15909-7 & \begin{tabular}{l|l|l|}
27323 & WVE15909 \\
\end{tabular} & $7 / 8 / 0822: 11$ & 444.3506 & 7 \\
\hline WVE15909-8 & 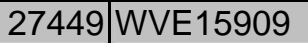 & 7/9/08 11:09 & 444.3756 & 8 \\
\hline WVE15909-9 & 27578 $\mid$ WVE15909 & 7/10/08 16:42 & 444.3969 & 9 \\
\hline WVE15909-10 & 27687 WVE15909 & 7/11/08 10:58 & 444.4047 & 10 \\
\hline WVE15909-11 & 27929|WVE15909 & 7/14/08 14:11 & 444.4395 & 11 \\
\hline WVE15909-12 & \begin{tabular}{|l|l|}
28248 & WVE15909 \\
\end{tabular} & 7/18/08 15:06 & 444.4713 & 12 \\
\hline WVE15909-13 & 28865 WVE15909 & $7 / 25 / 08$ 16:43 & 444.5079 & 13 \\
\hline WVE15909-14 & 29251 WVE15909 & $7 / 30 / 08$ 12:25 & 444.5294 & 14 \\
\hline WVE15909-15 & 29649 WVE15909 & 8/7/08 19:28 & 444.5582 & 15 \\
\hline WVE15910-1 & \begin{tabular}{l|l|l|}
26742 & WVE15910 \\
\end{tabular} & 7/2/08 15:08 & 424.783 & 1 \\
\hline WVE15910-2 & \begin{tabular}{|l|l|}
26849 & WVE15910 \\
\end{tabular} & $7 / 3 / 08$ 10:56 & 424.775 & 2 \\
\hline WVE15910-3 & \begin{tabular}{|l|l|}
26951 & WVE15910 \\
\end{tabular} & $7 / 3 / 08$ 15:37 & 424.7723 & 3 \\
\hline WVE15910-4 & 27058 $\mid$ WVE15910 & 7/7/08 10:29 & 424.7718 & 4 \\
\hline WVE15910-5 & $27105 \mid$ WVE15910 & 7/7/08 19:47 & 424.26 & 5 \\
\hline WVE15910-6 & \begin{tabular}{|l|l|}
27193 & WVE15910 \\
\end{tabular} & 7/8/08 10:28 & 424.3273 & 6 \\
\hline WVE15910-7 & \begin{tabular}{l|l|l}
27324 & WVE15910
\end{tabular} & $7 / 8 / 0822: 12$ & 424.3475 & 7 \\
\hline WVE15910-8 & \begin{tabular}{l|l|l|}
27450 & WVE15910 \\
\end{tabular} & 7/9/08 11:09 & 424.3649 & 8 \\
\hline WVE15910-9 & 27579 WVE15910 & 7/10/08 16:43 & 424.3877 & 9 \\
\hline WVE15910-10 & \begin{tabular}{|l|l|}
27688 & WVE15910 \\
\end{tabular} & 7/11/08 10:59 & 424.3994 & 10 \\
\hline WVE15910-11 & \begin{tabular}{l|l|l}
27930 & WVE15910 \\
\end{tabular} & 7/14/08 14:12 & 424.4333 & 11 \\
\hline WVE15910-12 & 28249 WVE15910 & 7/18/08 15:07 & 424.4655 & 12 \\
\hline WVE15910-13 & \begin{tabular}{|l|l|}
28866 & WVE15910 \\
\end{tabular} & 7/25/08 16:43 & 424.4948 & 13 \\
\hline WVE15910-14 & 29252 WVE15910 & 7/30/08 12:26 & 424.5146 & 14 \\
\hline WVE15910-15 & \begin{tabular}{l|l|l|}
29650 & WVE15910 \\
\end{tabular} & 8/7/08 19:29 & 424.5408 & 15 \\
\hline
\end{tabular}




\begin{tabular}{|c|c|c|c|c|}
\hline WVREF237-1 & 26630|WVREF237 & 7/2/08 13:10 & -0.0016 & 1 \\
\hline WVREF237-2 & \begin{tabular}{l|l|}
26631 & WVREF237
\end{tabular} & $7 / 2 / 08$ 13:14 & 0 & 2 \\
\hline WVREF237-3 & \begin{tabular}{l|l}
26633 & WVREF237
\end{tabular} & $7 / 2 / 08$ 13:17 & 0.0026 & 3 \\
\hline WVREF237-4 & \begin{tabular}{l|l|l}
26634 & WVREF237
\end{tabular} & 7/2/08 13:17 & 0 & 4 \\
\hline WVREF237-5 & 26636|WVREF237 & 7/2/08 13:18 & -0.0006 & 5 \\
\hline WVREF237-6 & \begin{tabular}{|l|l|}
26637 & WVREF237
\end{tabular} & 7/2/08 13:18 & 0 & 6 \\
\hline WVREF623-1 & 26646|WVREF623 & $7 / 2 / 08$ 13:27 & 299.8701 & 1 \\
\hline WVREF623-2 & 26749 WVREF623 & $7 / 3 / 089: 20$ & 299.8648 & 2 \\
\hline WVREF623-3 & 26854|WVREF623 & 7/3/08 14:03 & 299.872 & 3 \\
\hline WVREF623-4 & 26959 WVREF623 & $7 / 7 / 088: 54$ & 299.8605 & 4 \\
\hline WVREF623-5 & 27067 WVREF623 & $7 / 7 / 08$ 16:36 & 299.8594 & 5 \\
\hline WVREF623-6 & 27128 $\mid$ WVREF623 & $7 / 8 / 089: 29$ & 299.8606 & 6 \\
\hline WVREF623-7 & 27197 WVREF623 & $7 / 8 / 08$ 14:19 & 299.8643 & 7 \\
\hline WVREF623-8 & 27261 WVREF623 & $7 / 8 / 0820: 46$ & 299.8627 & 8 \\
\hline WVREF623-9 & \begin{tabular}{|l|l|}
27349 & WVREF623 \\
\end{tabular} & 7/9/08 9:41 & 299.8626 & 9 \\
\hline WVREF623-10 & 27480 WVREF623 & 7/10/08 14:57 & 299.8636 & 10 \\
\hline WVREF623-11 & 27587 WVREF623 & 7/11/08 9:32 & 299.8656 & 11 \\
\hline WVREF623-12 & \begin{tabular}{l|l}
27721 & WVREF623 \\
\end{tabular} & 7/11/08 18:19 & 299.8648 & 12 \\
\hline WVREF623-13 & \begin{tabular}{|l|l|}
27766 & WVREF623 \\
\end{tabular} & 7/12/08 13:00 & 299.8611 & 13 \\
\hline WVREF623-14 & 27798|WVREF623 & 7/13/08 14:58 & 299.8668 & 14 \\
\hline WVREF623-15 & \begin{tabular}{|l|l|}
27832 & WVREF623 \\
\end{tabular} & $7 / 14 / 08$ 12:09 & 299.8664 & 15 \\
\hline WVREF623-16 & 27980 WVREF623 & $7 / 15 / 08$ 13:22 & 299.8648 & 16 \\
\hline WVREF623-17 & \begin{tabular}{|l|l|}
28012 & WVREF623 \\
\end{tabular} & 7/15/08 19:11 & 299.8629 & 17 \\
\hline WVREF623-18 & 28075 WVREF623 & 7/16/08 19:14 & 299.8611 & 18 \\
\hline WVREF623-19 & 28109 WVREF623 & 7/17/08 14:35 & 299.862 & 19 \\
\hline WVREF623-20 & \begin{tabular}{l|l}
28151 & WVREF623 \\
\end{tabular} & 7/18/08 13:12 & 299.8611 & 20 \\
\hline WVREF623-21 & 28254|WVREF623 & $7 / 18 / 08$ 15:23 & 299.8636 & 21 \\
\hline WVREF623-22 & \begin{tabular}{|l|l|}
28386 & WVREF623 \\
\end{tabular} & 7/21/08 11:11 & 299.8634 & 22 \\
\hline WVREF623-23 & \begin{tabular}{l|l|l|}
28492 & WVREF623
\end{tabular} & $7 / 22 / 08$ 12:07| & 299.8646 & 23 \\
\hline WVREF623-24 & \begin{tabular}{|l|l|}
28492 & WVREF623
\end{tabular} & $7 / 22 / 08$ 12:07 & 299.8646 & 24 \\
\hline WVREF623-25 & \begin{tabular}{l|l|l}
28566 & WVREF623
\end{tabular} & $7 / 23 / 08$ 11:27| & 299.8661 & 25 \\
\hline WVREF623-26 & 28640 WVREF623 & $7 / 24 / 08$ 12:13 & 299.8643 & 26 \\
\hline WVREF623-27 & \begin{tabular}{l|l|}
28683 & WVREF623 \\
\end{tabular} & 7/24/08 18:05 & 299.8668 & 27 \\
\hline WVREF623-28 & 28714 WVREF623 & 7/25/08 13:03 & 299.8631 & 28 \\
\hline WVREF623-29 & \begin{tabular}{|l|l|}
28778 & WVREF623 \\
\end{tabular} & $7 / 25 / 08$ 15:08 & 299.8636 & 29 \\
\hline WVREF623-30 & 28905 WVREF623 & 7/26/08 14:57| & 299.8679 & 30 \\
\hline WVREF623-31 & 28973|WVREF623 & 7/27/08 14:15 & 299.8648 & 31 \\
\hline WVREF623-32 & 29024 WVREF623 & $7 / 28 / 08$ 18:11 & 299.8653 & 32 \\
\hline WVREF623-33 & 29086|WVREF623 & $7 / 29 / 08$ 12:10 & 299.8701 & 33 \\
\hline WVREF623-34 & 29092 WVREF623 & $7 / 29 / 08$ 12:14 & 299.8656 & 34 \\
\hline WVREF623-35 & \begin{tabular}{l|l|l|}
29147 & WVREF623 \\
\end{tabular} & 7/30/08 11:02 & 299.8692 & 35 \\
\hline WVREF623-36 & 29156 WVREF623 & 7/30/08 11:08 & 299.8671 & 36 \\
\hline WVREF623-37 & \begin{tabular}{|l|l|}
29283 & WVREF623 \\
\end{tabular} & 7/31/08 15:22 & 299.8729 & 37 \\
\hline WVREF623-38 & 29309 WVREF623 & 8/1/08 13:38 & 299.87 & 38 \\
\hline WVREF623-39 & 29374|WVREF623 & 8/2/08 14:23 & 299.8724 & 39 \\
\hline WVREF623-40 & 29416 WVREF623 & $8 / 3 / 08$ 12:27 & 299.8694 & 40 \\
\hline WVREF623-41 & \begin{tabular}{|l|l|}
29463 & WVREF623 \\
\end{tabular} & $8 / 4 / 08$ 12:56 & 299.8739 & 41 \\
\hline WVREF623-42 & 29509 WVREF623 & $8 / 5 / 08$ 12:56 & 299.873 & 42 \\
\hline WVREF623-43 & \begin{tabular}{l|l|l|}
29551 & WVREF623 \\
\end{tabular} & 8/7/08 17:16 & 299.8753 & 43 \\
\hline
\end{tabular}




\begin{tabular}{|c|c|c|c|c|}
\hline WVREF623-44 & \begin{tabular}{l|l|l|}
29840 & WVREF623 \\
\end{tabular} & $8 / 14 / 08$ 15:35 & 299.8827 & 44 \\
\hline WVREF623-45 & 30013|WVREF623 & 8/21/08 9:46 & 299.8774 & 45 \\
\hline WVREF623-46 & 30265|WVREF623 & 8/28/08 9:21 & 299.8826 & 46 \\
\hline WVREF623-47 & \begin{tabular}{l|l|l|}
30486 & WVREF623 \\
\end{tabular} & 9/3/08 14:25 & 299.8816 & 47 \\
\hline WVREF624-1 & \begin{tabular}{l|l|} 
26643 & WVREF624
\end{tabular} & 7/2/08 13:24 & 300.4059 & 1 \\
\hline WVREF624-2 & \begin{tabular}{l|l|l}
26750 & WVREF624
\end{tabular} & 7/3/08 9:21 & 300.4026 & 2 \\
\hline WVREF624-3 & \begin{tabular}{l|l|l}
26855 & WVREF624
\end{tabular} & $7 / 3 / 08$ 14:04 & 300.3813 & 3 \\
\hline WVREF624-4 & \begin{tabular}{l|l|} 
26960 & WVREF624
\end{tabular} & $7 / 7 / 088: 54$ & 300.3798 & 4 \\
\hline WVREF624-5 & \begin{tabular}{l|l|l}
27066 & WVREF624 \\
\end{tabular} & 7/7/08 16:35 & 300.3787 & 5 \\
\hline WVREF624-6 & \begin{tabular}{l|l|l}
27129 & WVREF624
\end{tabular} & $7 / 8 / 089: 30$ & 300.378 & 6 \\
\hline WVREF624-7 & \begin{tabular}{l|l}
27198 & WVREF624
\end{tabular} & $7 / 8 / 0814: 20$ & 300.3786 & 7 \\
\hline WVREF624-8 & \begin{tabular}{l|l}
27262 & WVREF624
\end{tabular} & $7 / 8 / 08$ 20:47 & 300.3803 & 8 \\
\hline WVREF624-9 & \begin{tabular}{l|l|l|}
27350 & WVREF624 \\
\end{tabular} & 7/9/08 9:42 & 300.3781 & 9 \\
\hline WVREF624-10 & \begin{tabular}{|l|l|}
27481 & WVREF624 \\
\end{tabular} & 7/10/08 14:58 & 300.3798 & 10 \\
\hline WVREF624-11 & \begin{tabular}{l|l|}
27588 & WVREF624 \\
\end{tabular} & 7/11/08 9:33 & 300.375 & 11 \\
\hline WVREF624-12 & \begin{tabular}{l|l|l|}
27722 & WVREF624 \\
\end{tabular} & $7 / 11 / 08$ 18:21 & 300.3763 & 12 \\
\hline WVREF624-13 & \begin{tabular}{l|l|}
27764 & WVREF624 \\
\end{tabular} & $7 / 12 / 08$ 12:58 & 300.3732 & 13 \\
\hline WVREF624-14 & 27799 $\mid$ WVREF624 & 7/13/08 14:58 & 300.3765 & 14 \\
\hline WVREF624-15 & \begin{tabular}{l|l|}
27831 & WVREF624 \\
\end{tabular} & $7 / 14 / 08$ 12:08 & 300.377 & 15 \\
\hline WVREF624-16 & \begin{tabular}{l|l|}
27981 & WVREF624
\end{tabular} & $7 / 15 / 08$ 13:23 & 300.378 & 16 \\
\hline WVREF624-17 & \begin{tabular}{l|l|}
28013 & WVREF624 \\
\end{tabular} & 7/15/08 19:11 & 300.377 & 17 \\
\hline WVREF624-18 & \begin{tabular}{l|l|}
28076 & WVREF624 \\
\end{tabular} & 7/16/08 19:15 & 300.3747 & 18 \\
\hline WVREF624-19 & \begin{tabular}{l|l|l}
28110 & WVREF624 \\
\end{tabular} & $7 / 17 / 08$ 14:36 & 300.3779 & 19 \\
\hline WVREF624-20 & \begin{tabular}{l|l|l|}
28152 & WVREF624 \\
\end{tabular} & 7/18/08 13:13 & 300.3737 & 20 \\
\hline WVREF624-21 & \begin{tabular}{l|l|l|}
28255 & WVREF624 \\
\end{tabular} & $7 / 18 / 08$ 15:25 & 300.3775 & 21 \\
\hline WVREF624-22 & \begin{tabular}{l|l|l|}
28387 & WVREF624 \\
\end{tabular} & 7/21/08 11:12 & 300.3774 & 22 \\
\hline WVREF624-23 & \begin{tabular}{|l|l|}
28493 & WVREF624 \\
\end{tabular} & $7 / 22 / 08$ 12:08 & 300.3788 & 23 \\
\hline WVREF624-24 & \begin{tabular}{l|l|l|}
28493 & WVREF624 \\
\end{tabular} & $7 / 22 / 08$ 12:08 & 300.3788 & 24 \\
\hline WVREF624-25 & \begin{tabular}{l|l|}
28567 & WVREF624
\end{tabular} & 7/23/08 11:27 & 300.3789 & 25 \\
\hline WVREF624-26 & \begin{tabular}{l|l|}
28641 & WVREF624 \\
\end{tabular} & $7 / 24 / 08$ 12:14 & 300.3777 & 26 \\
\hline WVREF624-27 & 28684|WVREF624 & 7/24/08 18:06 & 300.378 & 27 \\
\hline WVREF624-28 & \begin{tabular}{l|l|l}
28715 & WVREF624
\end{tabular} & 7/25/08 13:04 & 300.3738 & 28 \\
\hline WVREF624-29 & 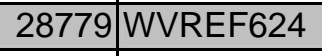 & 7/25/08 15:09 & 300.376 & 29 \\
\hline WVREF624-30 & \begin{tabular}{l|l|}
28902 & WVREF624
\end{tabular} & $7 / 26 / 08$ 14:54 & 300.3805 & 30 \\
\hline WVREF624-31 & \begin{tabular}{|l|l|}
28970 & WVREF624 \\
\end{tabular} & $7 / 27 / 08$ 14:13 & 300.3811 & 31 \\
\hline WVREF624-32 & \begin{tabular}{l|l|}
29025 & WVREF624 \\
\end{tabular} & $7 / 28 / 08$ 18:12 & 300.3805 & 32 \\
\hline WVREF624-33 & \begin{tabular}{l|l|}
29087 & WVREF624 \\
\end{tabular} & $7 / 29 / 08$ 12:11 & 300.3837 & 33 \\
\hline WVREF624-34 & 29093|WVREF624 & $7 / 29 / 08$ 12:15 & 300.3814 & 34 \\
\hline WVREF624-35 & \begin{tabular}{l|l|l|}
29148 & WVREF624 \\
\end{tabular} & 7/30/08 11:03 & 300.385 & 35 \\
\hline WVREF624-36 & \begin{tabular}{l|l|l|}
29155 & WVREF624 \\
\end{tabular} & $7 / 30 / 08$ 11:07 & 300.3823 & 36 \\
\hline WVREF624-37 & \begin{tabular}{l|l|}
29284 & WVREF624 \\
\end{tabular} & 7/31/08 15:22 & 300.385 & 37 \\
\hline WVREF624-38 & 29310 $\mid$ WVREF624 & 8/1/08 13:39 & 300.3859 & 38 \\
\hline WVREF624-39 & \begin{tabular}{l|l|}
29373 & WVREF624 \\
\end{tabular} & $8 / 2 / 08$ 14:22 & 300.3873 & 39 \\
\hline WVREF624-40 & \begin{tabular}{l|l|l|}
29417 & WVREF624 \\
\end{tabular} & $8 / 3 / 08$ 12:28 & 300.3836 & 40 \\
\hline WVREF624-41 & \begin{tabular}{l|l|}
29462 & WVREF624 \\
\end{tabular} & $8 / 4 / 0812: 55$ & 300.3848 & 41 \\
\hline WVREF624-42 & \begin{tabular}{|l|l|}
29510 & WVREF624 \\
\end{tabular} & $8 / 5 / 08$ 12:57 & 300.3857 & 42 \\
\hline WVREF624-43 & \begin{tabular}{l|l|}
29552 & WVREF624
\end{tabular} & 8/7/08 17:17 & 300.3847 & 43 \\
\hline WVREF624-44 & \begin{tabular}{l|l|l|}
29841 & WVREF624 \\
\end{tabular} & $8 / 14 / 08$ 15:36 & 300.3889 & 44 \\
\hline WVREF624-45 & \begin{tabular}{|l|l|}
30012 & WVREF624 \\
\end{tabular} & 8/21/08 9:45 & 300.3844 & 45 \\
\hline
\end{tabular}




\begin{tabular}{|c|c|c|c|c|}
\hline WVREF624-46 & \begin{tabular}{l|l|}
30266 & WVREF624
\end{tabular} & 8/28/08 9:21 & 300.3897 & 46 \\
\hline WVREF624-47 & \begin{tabular}{l|l}
30487 & WVREF624 \\
\end{tabular} & 9/3/08 14:26 & 300.3881 & 47 \\
\hline WVREF625-1 & \begin{tabular}{l|l|}
26642 & WVREF625
\end{tabular} & $7 / 2 / 08$ 13:22 & 433.304 & 1 \\
\hline WVREF625-2 & \begin{tabular}{l|l|l|}
26751 & WVREF625
\end{tabular} & $7 / 3 / 08$ 9:22 & 433.3023 & 2 \\
\hline WVREF625-3 & \begin{tabular}{l|l|l|}
26856 & WVREF625
\end{tabular} & 7/3/08 14:05 & 433.3018 & 3 \\
\hline WVREF625-4 & 26961|WVREF625 & 7/7/08 8:55 & 433.3005 & 4 \\
\hline WVREF625-5 & 27065|WVREF625 & 7/7/08 16:34 & 433.2947 & 5 \\
\hline WVREF625-6 & 27130|WVREF625 & 7/8/08 9:31 & 433.2992 & 6 \\
\hline WVREF625-7 & 27199 WVREF625 & $7 / 8 / 08$ 14:21 & 433.2971 & 7 \\
\hline WVREF625-8 & \begin{tabular}{l|l}
27263 & WVREF625
\end{tabular} & $7 / 8 / 0820: 48$ & 433.2982 & 8 \\
\hline WVREF625-9 & 27351 WVREF625 & 7/9/08 9:43 & 433.3002 & 9 \\
\hline WVREF625-10 & \begin{tabular}{l|l|l}
27482 & WVREF625
\end{tabular} & 7/10/08 14:59 & 433.2956 & 10 \\
\hline WVREF625-11 & 27589 WVREF625 & 7/11/08 9:34 & 433.2871 & 11 \\
\hline WVREF625-12 & \begin{tabular}{l|l}
27723 & WVREF625
\end{tabular} & $7 / 11 / 08$ 18:22 & 433.2928 & 12 \\
\hline WVREF625-13 & \begin{tabular}{l|l}
27763 & WVREF625
\end{tabular} & 7/12/08 12:57| & 433.2959 & 13 \\
\hline WVREF625-14 & \begin{tabular}{l|l|l|}
27797 & WVREF625
\end{tabular} & 7/13/08 14:57 & 433.295 & 14 \\
\hline WVREF625-15 & 27833|WVREF625 & $7 / 14 / 08$ 12:10 & 433.294 & 15 \\
\hline WVREF625-16 & 27983|WVREF625 & $7 / 15 / 08$ 13:25 & 433.2972 & 16 \\
\hline WVREF625-17 & $28014 \mid$ WVREF625 & 7/15/08 19:12 & 433.2943 & 17 \\
\hline WVREF625-18 & 28077 WVREF625 & 7/16/08 19:16 & 433.292 & 18 \\
\hline WVREF625-19 & 28111|WVREF625 & $7 / 17 / 08$ 14:36 & 433.2909 & 19 \\
\hline WVREF625-20 & 28153 WVREF625 & 7/18/08 13:14 & 433.2871 & 20 \\
\hline WVREF625-21 & \begin{tabular}{l|l|l}
28256 & WVREF625
\end{tabular} & 7/18/08 15:25 & 433.2942 & 21 \\
\hline WVREF625-22 & 28388 $\mid$ WVREF625 & 7/21/08 11:13 & 433.2931 & 22 \\
\hline WVREF625-23 & 28494|WVREF625 & $7 / 22 / 08$ 12:09 & 433.292 & 23 \\
\hline WVREF625-24 & 28494 WVREF625 & $7 / 22 / 08$ 12:09 & 433.292 & 24 \\
\hline WVREF625-25 & 28568 $\mid$ WVREF625 & $7 / 23 / 08$ 11:28 & 433.3018 & 25 \\
\hline WVREF625-26 & \begin{tabular}{l|l|l|}
28642 & WVREF625
\end{tabular} & 7/24/08 12:14 & 433.2982 & 26 \\
\hline WVREF625-27 & 28685 WVREF625 & $7 / 24 / 08$ 18:07| & 433.3002 & 27 \\
\hline WVREF625-28 & \begin{tabular}{|l|l|l|}
28716 & WVREF625
\end{tabular} & $7 / 25 / 08$ 13:04 & 433.293 & 28 \\
\hline WVREF625-29 & \begin{tabular}{|l|l|}
28780 & WVREF625 \\
\end{tabular} & 7/25/08 15:10 & 433.2975 & 29 \\
\hline WVREF625-30 & \begin{tabular}{|l|l|}
28903 & WVREF625
\end{tabular} & $7 / 26 / 08$ 14:55 & 433.3009 & 30 \\
\hline WVREF625-31 & 28971 WVREF625 & $7 / 27 / 08$ 14:13 & 433.2997 & 31 \\
\hline WVREF625-32 & \begin{tabular}{l|l|l}
29026 & WVREF625
\end{tabular} & 7/28/08 18:13 & 433.2994 & 32 \\
\hline WVREF625-33 & \begin{tabular}{|l|l|}
29088 & WVREF625 \\
\end{tabular} & $7 / 29 / 08$ 12:12 & 433.3055 & 33 \\
\hline WVREF625-34 & 29094 WVREF625 & 7/29/08 12:15 & 433.3024 & 34 \\
\hline WVREF625-35 & 29149 WVREF625 & 7/30/08 11:04 & 433.3091 & 35 \\
\hline WVREF625-36 & 29154 WVREF625 & $7 / 30 / 08$ 11:06 & 433.3046 & 36 \\
\hline WVREF625-37 & \begin{tabular}{l|l|l|}
29285 & WVREF625 \\
\end{tabular} & 7/31/08 15:23 & 433.3063 & 37 \\
\hline WVREF625-38 & 29311 WVREF625 & 8/1/08 13:39 & 433.3044 & 38 \\
\hline WVREF625-39 & \begin{tabular}{l|l|l|}
29372 & WVREF625
\end{tabular} & $8 / 2 / 0814: 22$ & 433.3068 & 39 \\
\hline WVREF625-40 & 29418 $\mid$ WVREF625 & 8/3/08 12:29 & 433.3038 & 40 \\
\hline WVREF625-41 & 29464|WVREF625 & $8 / 4 / 08$ 12:56 & 433.3059 & 41 \\
\hline WVREF625-42 & 29511 WVREF625 & 8/5/08 12:57 & 433.3061 & 42 \\
\hline WVREF625-43 & \begin{tabular}{l|l}
29553 & WVREF625
\end{tabular} & 8/7/08 17:18 & 433.3084 & 43 \\
\hline WVREF625-44 & \begin{tabular}{l|l|l}
29842 & WVREF625
\end{tabular} & 8/14/08 15:37 & 433.3193 & 44 \\
\hline WVREF625-45 & \begin{tabular}{l|l|l|}
30011 & WVREF625
\end{tabular} & 8/21/08 9:45 & 433.3164 & 45 \\
\hline WVREF625-46 & 30267 WVREF625 & 8/28/08 9:22 & 433.3218 & 46 \\
\hline WVREF625-47 & \begin{tabular}{|l|l|}
30488 & WVREF625 \\
\end{tabular} & 9/3/08 14:27 & 433.3281 & 47 \\
\hline
\end{tabular}




\begin{tabular}{|c|c|c|c|c|c|}
\hline WVREF626-1 & 26641 & WVREF626 & $7 / 2 / 08$ 13:21 & 433.2719 & 1 \\
\hline WVREF626-2 & 26752 & WVREF626 & $7 / 3 / 08$ 9:23 & 433.297 & 2 \\
\hline WVREF626-3 & 26857 & WVREF626 & $7 / 3 / 0814: 06$ & 433.2704 & 3 \\
\hline WVREF626-4 & 26962 & WVREF626 & $7 / 7 / 088: 56$ & 433.2638 & 4 \\
\hline WVREF626-5 & 27064 & WVREF626 & $7 / 7 / 08$ 16:33 & 433.2568 & 5 \\
\hline WVREF626-6 & 27131 & WVREF626 & 7/8/08 9:32 & 433.2585 & 6 \\
\hline WVREF626-7 & 27200 & WVREF626 & $7 / 8 / 08$ 14:21 & 433.2584 & 7 \\
\hline WVREF626-8 & 27264 & WVREF626 & $7 / 8 / 08$ 20:49 & 433.2587 & 8 \\
\hline WVREF626-9 & 27352 & WVREF626 & 7/9/08 9:43 & 433.264 & 9 \\
\hline WVREF626-10 & 27483 & WVREF626 & $7 / 10 / 08$ 15:00 & 433.267 & 10 \\
\hline WVREF626-11 & 27590 & WVREF626 & 7/11/08 9:34 & 433.2542 & 11 \\
\hline WVREF626-12 & 27724 & WVREF626 & $7 / 11 / 08$ 18:23 & 433.2622 & 12 \\
\hline WVREF626-13 & 27765 & WVREF626 & $7 / 12 / 08$ 12:59 & 433.252 & 13 \\
\hline WVREF626-14 & 27796 & WVREF626 & $7 / 13 / 08$ 14:57 & 433.2589 & 14 \\
\hline WVREF626-15 & 27834 & WVREF626 & $7 / 14 / 08$ 12:11 & 433.2557 & 15 \\
\hline WVREF626-16 & 27982 & WVREF626 & $7 / 15 / 08$ 13:24 & 433.2606 & 16 \\
\hline WVREF626-17 & 28015 & WVREF626 & $7 / 15 / 08$ 19:13 & 433.2552 & 17 \\
\hline WVREF626-18 & 28078 & WVREF626 & $7 / 16 / 08$ 19:17 & 433.2533 & 18 \\
\hline WVREF626-19 & 28112 & WVREF626 & $7 / 17 / 08$ 14:37 & 433.2587 & 19 \\
\hline WVREF626-20 & 28154 & WVREF626 & $7 / 18 / 08$ 13:15 & 433.2471 & 20 \\
\hline WVREF626-21 & 28257 & WVREF626 & $7 / 18 / 08$ 15:26 & 433.257 & 21 \\
\hline WVREF626-22 & 28389 & WVREF626 & $7 / 21 / 08$ 11:14 & 433.2553 & 22 \\
\hline WVREF626-23 & 28495 & WVREF626 & $7 / 22 / 08$ 12:10 & 433.253 & 23 \\
\hline WVREF626-24 & 28495 & WVREF626 & $7 / 22 / 08$ 12:10 & 433.253 & 24 \\
\hline WVREF626-25 & 28569 & WVREF626 & $7 / 23 / 08$ 11:29 & 433.2595 & 25 \\
\hline WVREF626-26 & 28643 & WVREF626 & $7 / 24 / 08$ 12:15 & 433.2588 & 26 \\
\hline WVREF626-27 & 28686 & WVREF626 & $7 / 24 / 08$ 18:08 & 433.2577 & 27 \\
\hline WVREF626-28 & 28717 & WVREF626 & $7 / 25 / 08$ 13:05 & 433.2571 & 28 \\
\hline WVREF626-29 & 28781 & WVREF626 & $7 / 25 / 08$ 15:11 & 433.2587 & 29 \\
\hline WVREF626-30 & 28904 & WVREF626 & $7 / 26 / 08$ 14:56 & 433.2639 & 30 \\
\hline WVREF626-31 & 28972 & WVREF626 & $7 / 27 / 08$ 14:14 & 433.2594 & 31 \\
\hline WVREF626-32 & 29027 & WVREF626 & $7 / 28 / 08$ 18:14 & 433.2607 & 32 \\
\hline WVREF626-33 & 29089 & WVREF626 & $7 / 29 / 08$ 12:12 & 433.2624 & 33 \\
\hline WVREF626-34 & 29095 & WVREF626 & $7 / 29 / 08$ 12:16 & 433.2634 & 34 \\
\hline WVREF626-35 & 29150 & WVREF626 & $7 / 30 / 08$ 11:04 & 433.2613 & 35 \\
\hline WVREF626-36 & 29153 & WVREF626 & $7 / 30 / 08$ 11:06 & 433.264 & 36 \\
\hline WVREF626-37 & 29286 & WVREF626 & $7 / 31 / 08$ 15:23 & 433.2664 & 37 \\
\hline WVREF626-38 & 29312 & WVREF626 & $8 / 1 / 08$ 13:40 & 433.2648 & 38 \\
\hline WVREF626-39 & 29371 & WVREF626 & $8 / 2 / 08$ 14:21 & 433.2639 & 39 \\
\hline WVREF626-40 & 29419 & WVREF626 & $8 / 3 / 08$ 12:30 & 433.264 & 40 \\
\hline WVREF626-41 & 29465 & WVREF626 & $8 / 4 / 0812: 57$ & 433.2643 & 41 \\
\hline WVREF626-42 & 29512 & WVREF626 & 8/5/08 12:58 & 433.264 & 42 \\
\hline WVREF626-43 & 29554 & WVREF626 & 8/7/08 17:19 & 433.2682 & 43 \\
\hline WVREF626-44 & 29843 & WVREF626 & $8 / 14 / 08$ 15:37 & 433.2761 & 44 \\
\hline WVREF626-45 & 30010 & WVREF626 & $8 / 21 / 08$ 9:44 & 433.2785 & 45 \\
\hline WVREF626-46 & 30268 & WVREF626 & 8/28/08 9:23 & 433.2882 & 46 \\
\hline WVREF626-47 & 30489 & WVREF626 & $9 / 3 / 0814: 27$ & 433.2926 & 47 \\
\hline WVREF627-1 & 26638 & WVREF627 & $7 / 2 / 08$ 13:19 & 0 & 1 \\
\hline WVREF627-2 & 26639 & WVREF627 & $7 / 2 / 08$ 13:19 & 0 & 2 \\
\hline
\end{tabular}




\begin{tabular}{|c|c|c|c|c|}
\hline WVREF627-3 & \begin{tabular}{l|l|} 
26640 & WVREF627
\end{tabular} & 7/2/08 13:20 & 0 & 3 \\
\hline WVREF627-4 & \begin{tabular}{l|l|l}
26644 & WVREF627
\end{tabular} & $7 / 2 / 08$ 13:25 & 0.0027 & 4 \\
\hline WVREF627-5 & \begin{tabular}{l|l|l|}
26645 & WVREF627
\end{tabular} & $7 / 2 / 08$ 13:26 & 0 & 5 \\
\hline WVREF627-6 & \begin{tabular}{l|l|}
26647 & WVREF627
\end{tabular} & 7/2/08 13:27 & 0.0006 & 6 \\
\hline WVREF627-7 & 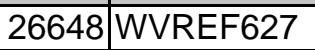 & $7 / 2 / 08$ 13:30 & 0 & 7 \\
\hline WVREF627-8 & \begin{tabular}{|c|l}
26659 & WVREF627
\end{tabular} & $7 / 2 / 08$ 13:40 & -0.001 & 8 \\
\hline WVREF627-9 & \begin{tabular}{l|l|l}
26660 & WVREF627
\end{tabular} & 7/2/08 13:43 & 0 & 9 \\
\hline WVREF627-10 & \begin{tabular}{l|l|}
26671 & WVREF627
\end{tabular} & 7/2/08 13:53 & 0.0014 & 10 \\
\hline WVREF627-11 & \begin{tabular}{|l|l|}
26672 & WVREF627 \\
\end{tabular} & 7/2/08 13:54 & 0 & 11 \\
\hline WVREF627-12 & \begin{tabular}{|l|l|}
26683 & WVEF627
\end{tabular} & $7 / 2 / 08$ 14:03 & 0.0002 & 12 \\
\hline WVREF627-13 & 26684 $\mid$ WVREF627 & $7 / 2 / 08$ 14:06 & 0 & 13 \\
\hline WVREF627-14 & \begin{tabular}{l|l|l}
26695 & WVREF627
\end{tabular} & $7 / 2 / 0814: 14$ & -0.0006 & 14 \\
\hline WVREF627-15 & \begin{tabular}{l|l|l}
26696 & WVREF627
\end{tabular} & $7 / 2 / 0814: 17$ & 0 & 15 \\
\hline WVREF627-16 & \begin{tabular}{l|l|}
26707 & WVREF627
\end{tabular} & $7 / 2 / 0814: 27$ & 0.0007 & 16 \\
\hline WVREF627-17 & \begin{tabular}{l|l|l}
26708 & WVREF627
\end{tabular} & $7 / 2 / 0814: 30$ & 0 & 17 \\
\hline WVREF627-18 & \begin{tabular}{l|l}
26719 & WVREF627
\end{tabular} & $7 / 2 / 08$ 14:41 & 0.0004 & 18 \\
\hline WVREF627-19 & 26720 WVREF627 & $7 / 2 / 08$ 14:44 & 0 & 19 \\
\hline WVREF627-20 & \begin{tabular}{l|l}
26731 & WVREF627 \\
\end{tabular} & $7 / 2 / 08$ 14:56 & -0.0004 & 20 \\
\hline WVREF627-21 & \begin{tabular}{l|l|}
26732 & WVREF627
\end{tabular} & $7 / 2 / 08$ 14:58 & 0 & 21 \\
\hline WVREF627-22 & \begin{tabular}{l|l}
26743 & WVREF627 \\
\end{tabular} & 7/2/08 15:08 & 0.0024 & 22 \\
\hline WVREF627-23 & 26744|WVREF627 & $7 / 3 / 08$ 9:12 & -0.0018 & 23 \\
\hline WVREF627-24 & \begin{tabular}{l|l|l}
26745 & WVREF627 \\
\end{tabular} & $7 / 3 / 089: 16$ & 0 & 24 \\
\hline WVREF627-25 & \begin{tabular}{l|l|}
26747 & WVREF627 \\
\end{tabular} & $7 / 3 / 089: 17$ & 0.0004 & 25 \\
\hline WVREF627-26 & \begin{tabular}{l|l|}
26748 & WVREF627
\end{tabular} & 7/3/08 9:17 & 0 & 26 \\
\hline WVREF627-27 & \begin{tabular}{l|l|}
26753 & WVREF627
\end{tabular} & 7/3/08 9:23 & -0.0011 & 27 \\
\hline WVREF627-28 & 26754 WVREF627 & $7 / 3 / 089: 24$ & 0 & 28 \\
\hline WVREF627-29 & \begin{tabular}{l|l|l|}
26755 & WVREF627
\end{tabular} & $7 / 3 / 089: 24$ & 0 & 29 \\
\hline WVREF627-30 & \begin{tabular}{l|l|l}
26766 & WVREF627
\end{tabular} & 7/3/08 9:33 & 0.0002 & 30 \\
\hline WVREF627-31 & \begin{tabular}{l|l}
26767 & WVREF627 \\
\end{tabular} & 7/3/08 9:35 & 0 & 31 \\
\hline WVREF627-32 & \begin{tabular}{l|l|l}
26778 & WVREF627
\end{tabular} & $7 / 3 / 089: 44$ & -0.0001 & 32 \\
\hline WVREF627-33 & \begin{tabular}{|l|l|}
26779 & WVREF627 \\
\end{tabular} & 7/3/08 9:46 & 0 & 33 \\
\hline WVREF627-34 & \begin{tabular}{|l|l|}
26790 & WVREF627
\end{tabular} & $7 / 3 / 089: 55$ & 0.0004 & 34 \\
\hline WVREF627-35 & \begin{tabular}{|l|l|}
26791 & WVREF627 \\
\end{tabular} & 7/3/08 9:57 & 0 & 35 \\
\hline WVREF627-36 & \begin{tabular}{l|l|}
26802 & WVREF627
\end{tabular} & 7/3/08 10:08 & 0.0009 & 36 \\
\hline WVREF627-37 & \begin{tabular}{|l|l|}
26803 & WVREF627 \\
\end{tabular} & 7/3/08 10:09 & 0 & 37 \\
\hline WVREF627-38 & \begin{tabular}{l|l}
26814 & WVREF627 \\
\end{tabular} & $7 / 3 / 08$ 10:20 & 0.0001 & 38 \\
\hline WVREF627-39 & \begin{tabular}{l|l|l|}
26815 & WVREF627 \\
\end{tabular} & 7/3/08 10:23 & 0 & 39 \\
\hline WVREF627-40 & \begin{tabular}{l|l|}
26826 & WVREF627 \\
\end{tabular} & $7 / 3 / 08$ 10:33 & 0.0009 & 40 \\
\hline WVREF627-41 & \begin{tabular}{l|l|}
26827 & WVREF627 \\
\end{tabular} & 7/3/08 10:36 & 0 & 41 \\
\hline WVREF627-42 & \begin{tabular}{|l|l|}
26838 & WVREF627 \\
\end{tabular} & $7 / 3 / 08$ 10:46 & -0.0001 & 42 \\
\hline WVREF627-43 & \begin{tabular}{l|l}
26839 & WVREF627
\end{tabular} & $7 / 3 / 08$ 10:47 & 0 & 43 \\
\hline WVREF627-44 & \begin{tabular}{l|l|l}
26850 & WVREF627
\end{tabular} & 7/3/08 10:58 & 0.001 & 44 \\
\hline WVREF627-45 & \begin{tabular}{l|l|l}
26851 & WVREF627
\end{tabular} & 7/3/08 14:01 & -0.0004 & 45 \\
\hline WVREF627-46 & \begin{tabular}{|l|l|}
26852 & WVREF627 \\
\end{tabular} & 7/3/08 14:02 & 0 & 46 \\
\hline WVREF627-47 & \begin{tabular}{l|l|l}
26853 & WVREF627
\end{tabular} & $7 / 3 / 08$ 14:02 & 0 & 47 \\
\hline WVREF627-48 & \begin{tabular}{|l|l|}
26858 & WVREF627 \\
\end{tabular} & 7/3/08 14:06 & 0.0003 & 48 \\
\hline WVREF627-49 & \begin{tabular}{l|l|}
26859 & WVREF627
\end{tabular} & 7/3/08 14:08 & 0 & 49 \\
\hline WVREF627-50 & \begin{tabular}{l|l}
26870 & WVREF627 \\
\end{tabular} & $7 / 3 / 08$ 14:15 & 0.0003 & 50 \\
\hline WVREF627-51 & \begin{tabular}{l|l|}
26871 & WVREF627 \\
\end{tabular} & $7 / 3 / 0814: 18$ & 0 & 51 \\
\hline
\end{tabular}




\begin{tabular}{|c|c|c|c|c|c|}
\hline WVREF627-52 & 26882 & WVREF627 & $7 / 3 / 08$ 14:27 & 0.0005 & 52 \\
\hline WVREF627-53 & 26883 & WVREF627 & $7 / 3 / 08$ 14:28 & 0 & 53 \\
\hline WVREF627-54 & 26894 & WVREF627 & 7/3/08 14:38 & 0.0006 & 54 \\
\hline WVREF627-55 & 26895 & WVREF627 & $7 / 3 / 08$ 14:38 & 0 & 55 \\
\hline WVREF627-56 & 26906 & WVREF627 & $7 / 3 / 08$ 14:50 & 0 & 56 \\
\hline WVREF627-57 & 26917 & WVREF627 & $7 / 3 / 08$ 14:59 & 0.0012 & 57 \\
\hline WVREF627-58 & 26918 & WVREF627 & $7 / 3 / 08$ 15:04 & 0 & 58 \\
\hline WVREF627-59 & 26929 & WVREF627 & $7 / 3 / 08$ 15:15 & 0.0001 & 59 \\
\hline WVREF627-60 & 26930 & WVREF627 & $7 / 3 / 08$ 15:15 & 0 & 60 \\
\hline WVREF627-61 & 26941 & WVREF627 & $7 / 3 / 08$ 15:27 & 0.0012 & 61 \\
\hline WVREF627-62 & 26942 & WVREF627 & $7 / 3 / 08$ 15:27 & 0 & 62 \\
\hline WVREF627-63 & 26953 & WVREF627 & $7 / 3 / 08$ 15:38 & -0.0007 & 63 \\
\hline WVREF627-64 & 26954 & WVREF627 & $7 / 7 / 088: 44$ & -0.0028 & 64 \\
\hline WVREF627-65 & 26955 & WVREF627 & 7/7/08 8:50 & 0 & 65 \\
\hline WVREF627-66 & 26957 & WVREF627 & 7/7/08 8:52 & 0.0025 & 66 \\
\hline WVREF627-67 & 26958 & WVREF627 & 7/7/08 8:52 & 0 & 67 \\
\hline WVREF627-68 & 26963 & WVREF627 & 7/7/08 8:58 & 0.0005 & 68 \\
\hline WVREF627-69 & 26964 & WVREF627 & 7/7/08 8:58 & 0 & 69 \\
\hline WVREF627-70 & 26975 & WVREF627 & 7/7/08 9:08 & 0.001 & 70 \\
\hline WVREF627-71 & 26976 & WVREF627 & 7/7/08 9:10 & 0 & 71 \\
\hline WVREF627-72 & 26987 & WVREF627 & 7/7/08 9:19 & 0.0016 & 72 \\
\hline WVREF627-73 & 26988 & WVREF627 & 7/7/08 9:22 & 0.0012 & 73 \\
\hline WVREF627-74 & 26989 & WVREF627 & 7/7/08 9:22 & 0 & 74 \\
\hline WVREF627-75 & 27000 & WVREF627 & 7/7/08 9:31 & -0.0001 & 75 \\
\hline WVREF627-76 & 27001 & WVREF627 & 7/7/08 9:33 & 0 & 76 \\
\hline WVREF627-77 & 27012 & WVREF627 & 7/7/08 9:42 & -0.0006 & 77 \\
\hline \begin{tabular}{|l|} 
WVREF627-78 \\
\end{tabular} & 27013 & WVREF627 & 7/7/08 9:43 & 0 & 78 \\
\hline WVREF627-79 & 27024 & WVREF627 & 7/7/08 9:56 & 0.0009 & 79 \\
\hline WVREF627-80 & 27025 & WVREF627 & 7/7/08 9:56 & 0 & 80 \\
\hline WVREF627-81 & 27036 & WVREF627 & $7 / 7 / 08$ 10:07 & 0 & 81 \\
\hline WVREF627-82 & 27047 & WVREF627 & $7 / 7 / 08$ 10:18 & 0.0012 & 82 \\
\hline WVREF627-83 & 27048 & WVREF627 & $7 / 7 / 08$ 10:20 & 0 & 83 \\
\hline WVREF627-84 & 27059 & WVREF627 & $7 / 7 / 08$ 10:30 & 0.0003 & 84 \\
\hline WVREF627-85 & 27060 & WVREF627 & $7 / 7 / 08$ 16:32 & 0 & 85 \\
\hline WVREF627-86 & 27062 & WVREF627 & $7 / 7 / 08$ 16:33 & 0.0002 & 86 \\
\hline WVREF627-87 & 27063 & WVREF627 & $7 / 7 / 08$ 16:33 & 0 & 87 \\
\hline WVREF627-88 & 27068 & WVREF627 & $7 / 7 / 08$ 16:36 & -0.0004 & 88 \\
\hline WVREF627-89 & 27069 & WVREF627 & 7/7/08 17:44 & 0 & 89 \\
\hline WVREF627-90 & 27075 & WVREF627 & $7 / 7 / 08$ 17:49 & 0.0005 & 90 \\
\hline WVREF627-91 & 27076 & WVREF627 & 7/7/08 17:49 & 0 & 91 \\
\hline WVREF627-92 & 27082 & WVREF627 & $7 / 7 / 08$ 17:53 & -0.0017 & 92 \\
\hline WVREF627-93 & 27083 & WVREF627 & $7 / 7 / 0817: 53$ & 0 & 93 \\
\hline WVREF627-94 & 27089 & WVREF627 & $7 / 7 / 08$ 17:58 & 0.0006 & 94 \\
\hline WVREF627-95 & 27090 & WVREF627 & 7/7/08 17:58 & 0 & 95 \\
\hline WVREF627-96 & 27096 & WVREF627 & $7 / 7 / 08$ 18:02 & -0.0001 & 96 \\
\hline WVREF627-97 & 27097 & WVREF627 & 7/7/08 19:41 & 0 & 97 \\
\hline WVREF627-98 & 27106 & WVREF627 & $7 / 7 / 08$ 19:48 & -0.0002 & 98 \\
\hline WVREF627-99 & 27107 & WVREF627 & 7/7/08 19:48 & 0 & 99 \\
\hline WVREF627-100 & 27115 & WVREF627 & $7 / 7 / 08$ 19:54 & 0.0003 & 100 \\
\hline
\end{tabular}




\begin{tabular}{|c|c|c|c|c|}
\hline WVREF627-101 & \begin{tabular}{l|l|l}
27116 & WVREF627
\end{tabular} & 7/7/08 19:54 & 0 & 101 \\
\hline WVREF627-102 & \begin{tabular}{l|l}
27122 & WVREF627 \\
\end{tabular} & 7/7/08 19:57 & 0.0003 & 102 \\
\hline WVREF627-103 & \begin{tabular}{l|l}
27123 & WVREF627
\end{tabular} & 7/8/08 9:22 & -0.0023 & 103 \\
\hline WVREF627-104 & 27124 WVREF627 & 7/8/08 9:27 & 0 & 104 \\
\hline WVREF627-105 & 27126|WVREF627 & 7/8/08 9:28 & 0.0003 & 105 \\
\hline WVREF627-106 & \begin{tabular}{l|l}
27127 & WVREF627
\end{tabular} & 7/8/08 9:29 & 0 & 106 \\
\hline WVREF627-107 & \begin{tabular}{|l|l|}
27132 & WVREF627 \\
\end{tabular} & 7/8/08 9:33 & 0.0007 & 107 \\
\hline WVREF627-108 & \begin{tabular}{l|l|l|} 
27133 & WVEF627
\end{tabular} & 7/8/08 9:34 & 0 & 108 \\
\hline WVREF627-109 & 27144 WVREF627 & $7 / 8 / 08$ 9:42 & 0.0001 & 109 \\
\hline WVREF627-110 & 27145 WVREF627 & $7 / 8 / 089: 45$ & 0 & 110 \\
\hline WVREF627-111 & \begin{tabular}{|l|l|}
27156 & WVREF627 \\
\end{tabular} & 7/8/08 9:53 & 0.0008 & 111 \\
\hline WVREF627-112 & \begin{tabular}{l|l}
27157 & WVREF627 \\
\end{tabular} & $7 / 8 / 089: 55$ & 0 & 112 \\
\hline WVREF627-113 & 27163 WVREF627 & 7/8/08 9:59 & 0.0006 & 113 \\
\hline WVREF627-114 & 27164 WVREF627 & 7/8/08 10:01 & 0 & 114 \\
\hline WVREF627-115 & 27175 WVREF627 & 7/8/08 10:10 & 0.0008 & 115 \\
\hline WVREF627-116 & \begin{tabular}{l|l|l}
27176 & WVREF627
\end{tabular} & 7/8/08 10:12 & 0 & 116 \\
\hline WVREF627-117 & 27187 $\mid$ WVREF627 & 7/8/08 10:24 & 0.0003 & 117 \\
\hline WVREF627-118 & \begin{tabular}{l|l}
27188 & WVREF627 \\
\end{tabular} & $7 / 8 / 08$ 10:24 & 0 & 118 \\
\hline WVREF627-119 & 27194 $\mid$ WVREF627 & 7/8/08 10:29 & 0.0007 & 119 \\
\hline WVREF627-120 & 27195 WVREF627 & 7/8/08 14:16 & 0.0006 & 120 \\
\hline WVREF627-121 & \begin{tabular}{l|l|l|}
27196 & WVREF627 \\
\end{tabular} & 7/8/08 14:19 & 0 & 121 \\
\hline WVREF627-122 & \begin{tabular}{l|l}
27201 & WVREF627
\end{tabular} & 7/8/08 14:22 & 0.0003 & 122 \\
\hline WVREF627-123 & \begin{tabular}{|l|l|}
27202 & WVREF627 \\
\end{tabular} & 7/8/08 14:30 & 0 & 123 \\
\hline WVREF627-124 & \begin{tabular}{l|l}
27213 & WVREF627 \\
\end{tabular} & 7/8/08 14:41 & -0.0004 & 124 \\
\hline WVREF627-125 & 27214 WVREF627 & 7/8/08 14:45 & 0 & 125 \\
\hline WVREF627-126 & \begin{tabular}{l|l}
27225 & WVREF627
\end{tabular} & 7/8/08 14:55 & 0.0004 & 126 \\
\hline WVREF627-127 & \begin{tabular}{|l|l|}
27226 & WVREF627
\end{tabular} & 7/8/08 14:55 & 0 & 127 \\
\hline WVREF627-128 & 27237 WVREF627 & 7/8/08 15:06 & 0.0002 & 128 \\
\hline WVREF627-129 & \begin{tabular}{l|l|l|}
27259 & WVREF627 \\
\end{tabular} & $7 / 8 / 0820: 43$ & -0.0037 & 129 \\
\hline WVREF627-130 & 27260 WVREF627 & $7 / 8 / 0820: 45$ & 0 & 130 \\
\hline WVREF627-131 & 27265 WVREF627 & $7 / 8 / 0820: 50$ & 0.0002 & 131 \\
\hline WVREF627-132 & \begin{tabular}{l|l|l}
27266 & WVREF627
\end{tabular} & 7/8/08 20:52 & 0 & 132 \\
\hline WVREF627-133 & 27277 WVREF627 & 7/8/08 21:07 & 0.0006 & 133 \\
\hline WVREF627-134 & \begin{tabular}{l|l}
27278 & WVREF627 \\
\end{tabular} & $7 / 8 / 0821: 07$ & 0 & 134 \\
\hline WVREF627-135 & \begin{tabular}{|l|l|}
27289 & WVREF627 \\
\end{tabular} & 7/8/08 21:26 & 0.0007 & 135 \\
\hline WVREF627-136 & \begin{tabular}{l|l}
27290 & WVREF627 \\
\end{tabular} & $7 / 8 / 0821: 26$ & 0 & 136 \\
\hline WVREF627-137 & \begin{tabular}{l|l|l}
27301 & WVREF627 \\
\end{tabular} & 7/8/08 21:44 & -0.0001 & 137 \\
\hline WVREF627-138 & \begin{tabular}{l|l|l}
27302 & WVREF627 \\
\end{tabular} & 7/8/08 21:44 & 0 & 138 \\
\hline WVREF627-139 & 27313 WVREF627 & 7/8/08 22:01 & -0.0013 & 139 \\
\hline WVREF627-140 & 27314 WVREF627 & $7 / 8 / 08$ 22:01 & 0 & 140 \\
\hline WVREF627-141 & \begin{tabular}{l|l|l}
27325 & WVREF627
\end{tabular} & $7 / 8 / 0822: 15$ & 0.0001 & 141 \\
\hline WVREF627-142 & \begin{tabular}{|l|l|}
27326 & WVREF627 \\
\end{tabular} & $7 / 8 / 0822: 16$ & 0.0001 & 142 \\
\hline WVREF627-143 & \begin{tabular}{l|l}
27341 & WVREF627
\end{tabular} & 7/9/08 9:29 & -0.0049 & 143 \\
\hline WVREF627-144 & \begin{tabular}{l|l|}
27342 & WVREF627 \\
\end{tabular} & 7/9/08 9:34 & 0 & 144 \\
\hline WVREF627-145 & \begin{tabular}{l|l|l}
27344 & WVREF627
\end{tabular} & 7/9/08 9:37 & 0.0027 & 145 \\
\hline WVREF627-146 & 27345 WVREF627 & 7/9/08 9:38 & 0 & 146 \\
\hline WVREF627-147 & \begin{tabular}{l|l}
27347 & WVREF627 \\
\end{tabular} & 7/9/08 9:40 & -0.001 & 147 \\
\hline WVREF627-148 & \begin{tabular}{l|l|}
27348 & WVREF627 \\
\end{tabular} & 7/9/08 9:40 & 0 & 148 \\
\hline WVREF627-149 & \begin{tabular}{|l|l|}
27353 & WVREF627 \\
\end{tabular} & $7 / 9 / 08$ 9:44 & -0.0002 & 149 \\
\hline
\end{tabular}




\begin{tabular}{|c|c|c|c|c|c|}
\hline WVREF627-150 & 27354 & WVREF627 & 7/9/08 9:45 & 0 & 150 \\
\hline WVREF627-151 & 27365 & WVREF627 & 7/9/08 9:54 & -0.0008 & 151 \\
\hline WVREF627-152 & 27366 & WVREF627 & 7/9/08 9:55 & 0 & 152 \\
\hline WVREF627-153 & 27368 & WVREF627 & 7/9/08 9:57 & 0.0014 & 153 \\
\hline WVREF627-154 & 27369 & WVREF627 & 7/9/08 9:57 & 0 & 154 \\
\hline WVREF627-155 & 27379 & WVREF627 & 7/9/08 10:05 & 0.0005 & 155 \\
\hline WVREF627-156 & 27380 & WVREF627 & $7 / 9 / 08$ 10:07 & 0 & 156 \\
\hline WVREF627-157 & 27391 & WVREF627 & $7 / 9 / 08$ 10:15 & 0.0004 & 157 \\
\hline WVREF627-158 & 27392 & WVREF627 & $7 / 9 / 08$ 10:17 & 0 & 158 \\
\hline WVREF627-159 & 27403 & WVREF627 & $7 / 9 / 08$ 10:25 & 0.0006 & 159 \\
\hline WVREF627-160 & 27404 & WVREF627 & $7 / 9 / 08$ 10:28 & 0 & 160 \\
\hline WVREF627-161 & 27415 & WVREF627 & $7 / 9 / 08$ 10:37 & -0.0002 & 161 \\
\hline WVREF627-162 & 27416 & WVREF627 & $7 / 9 / 08$ 10:39 & 0 & 162 \\
\hline WVREF627-163 & 27427 & WVREF627 & $7 / 9 / 08$ 10:49 & 0.0009 & 163 \\
\hline WVREF627-164 & 27428 & WVREF627 & 7/9/08 10:50 & 0 & 164 \\
\hline WVREF627-165 & 27439 & WVREF627 & $7 / 9 / 08$ 10:59 & 0.0003 & 165 \\
\hline WVREF627-166 & 27440 & WVREF627 & $7 / 9 / 08$ 11:01 & 0 & 166 \\
\hline WVREF627-167 & 27451 & WVREF627 & 7/9/08 11:10 & 0.0005 & 167 \\
\hline WVREF627-168 & 27458 & WVREF627 & $7 / 10 / 08$ 12:29 & 0.0026 & 168 \\
\hline WVREF627-169 & 27459 & WVREF627 & $7 / 10 / 08$ 12:34 & 0 & 169 \\
\hline WVREF627-170 & 27461 & WVREF627 & $7 / 10 / 08$ 12:37 & -0.0004 & 170 \\
\hline WVREF627-171 & 27462 & WVREF627 & $7 / 10 / 08$ 12:38 & -0.0003 & 171 \\
\hline WVREF627-172 & 27463 & WVREF627 & $7 / 10 / 08$ 12:42 & 0 & 172 \\
\hline WVREF627-173 & 27465 & WVREF627 & $7 / 10 / 08$ 12:44 & 0.0005 & 173 \\
\hline WVREF627-174 & 27478 & WVREF627 & $7 / 10 / 08$ 14:55 & 0.0006 & 174 \\
\hline WVREF627-175 & 27479 & WVREF627 & $7 / 10 / 08$ 14:56 & 0 & 175 \\
\hline WVREF627-176 & 27484 & WVREF627 & $7 / 10 / 08$ 15:02 & -0.0026 & 176 \\
\hline WVREF627-177 & 27485 & WVREF627 & $7 / 10 / 08$ 15:03 & 0 & 177 \\
\hline WVREF627-178 & 27496 & WVREF627 & $7 / 10 / 08$ 15:16 & 0.0005 & 178 \\
\hline WVREF627-179 & 27497 & WVREF627 & 7/10/08 15:16 & 0 & 179 \\
\hline WVREF627-180 & 27508 & WVREF627 & $7 / 10 / 08$ 15:29 & -0.0013 & 180 \\
\hline WVREF627-181 & 27509 & WVREF627 & $7 / 10 / 08$ 15:30 & 0 & 181 \\
\hline WVREF627-182 & 27520 & WVREF627 & $7 / 10 / 08$ 15:42 & -0.0003 & 182 \\
\hline WVREF627-183 & 27521 & WVREF627 & $7 / 10 / 08$ 15:42 & 0 & 183 \\
\hline WVREF627-184 & 27532 & WVREF627 & $7 / 10 / 08$ 15:54 & -0.0002 & 184 \\
\hline WVREF627-185 & 27533 & WVREF627 & $7 / 10 / 08$ 15:54 & 0 & 185 \\
\hline WVREF627-186 & 27544 & WVREF627 & 7/10/08 16:06 & -0.0008 & 186 \\
\hline WVREF627-187 & 27545 & WVREF627 & $7 / 10 / 08$ 16:06 & 0 & 187 \\
\hline WVREF627-188 & 27556 & WVREF627 & $7 / 10 / 08$ 16:20 & 0.0013 & 188 \\
\hline WVREF627-189 & 27557 & WVREF627 & $7 / 10 / 08$ 16:20 & 0 & 189 \\
\hline WVREF627-190 & 27568 & WVREF627 & 7/10/08 16:32 & -0.0009 & 190 \\
\hline WVREF627-191 & 27569 & WVREF627 & $7 / 10 / 08$ 16:32 & 0 & 191 \\
\hline WVREF627-192 & 27580 & WVREF627 & $7 / 10 / 08$ 16:45 & 0.0004 & 192 \\
\hline WVREF627-193 & 27581 & WVREF627 & 7/11/08 9:25 & -0.0018 & 193 \\
\hline WVREF627-194 & 27582 & WVREF627 & 7/11/08 9:30 & -0.0001 & 194 \\
\hline WVREF627-195 & 27583 & WVREF627 & 7/11/08 9:30 & 0 & 195 \\
\hline WVREF627-196 & 27585 & WVREF627 & 7/11/08 9:31 & -0.0002 & 196 \\
\hline WVREF627-197 & 27586 & WVREF627 & 7/11/08 9:31 & 0 & 197 \\
\hline WVREF627-198 & 27591 & WVREF627 & $7 / 11 / 089: 35$ & 0.0007 & 198 \\
\hline
\end{tabular}




\begin{tabular}{|c|c|c|c|c|}
\hline WVREF627-199 & \begin{tabular}{l|l|}
27592 & WVREF627
\end{tabular} & 7/11/08 9:36 & 0 & 199 \\
\hline WVREF627-200 & \begin{tabular}{l|l|}
27603 & WVREF627
\end{tabular} & 7/11/08 9:45 & -0.0004 & 200 \\
\hline WVREF627-201 & \begin{tabular}{l|l|l|}
27604 & WVREF627
\end{tabular} & 7/11/08 9:46 & 0 & 201 \\
\hline WVREF627-202 & \begin{tabular}{l|l|l}
27609 & WVREF627
\end{tabular} & 7/11/08 9:51 & 0.003 & 202 \\
\hline WVREF627-203 & \begin{tabular}{l|l|l}
27610 & WVREF627
\end{tabular} & 7/11/08 9:51 & 0 & 203 \\
\hline WVREF627-204 & \begin{tabular}{l|l|l}
27617 & WVREF627
\end{tabular} & 7/11/08 9:57| & -0.0001 & 204 \\
\hline WVREF627-205 & \begin{tabular}{l|l|l|}
27618 & WVREF627
\end{tabular} & 7/11/08 9:58 & 0 & 205 \\
\hline WVREF627-206 & \begin{tabular}{l|l}
27629 & WVREF627
\end{tabular} & 7/11/08 10:06 & 0.001 & 206 \\
\hline WVREF627-207 & \begin{tabular}{|l|l|}
27630 & WVREF627 \\
\end{tabular} & 7/11/08 10:08 & 0 & 207 \\
\hline WVREF627-208 & \begin{tabular}{l|l}
27641 & WVREF627
\end{tabular} & $7 / 11 / 08$ 10:16 & 0.0005 & 208 \\
\hline WVREF627-209 & \begin{tabular}{|l|l|}
27642 & WVREF627
\end{tabular} & 7/11/08 10:18 & 0 & 209 \\
\hline WVREF627-210 & \begin{tabular}{l|l|l|}
27653 & WVREF627
\end{tabular} & $7 / 11 / 08$ 10:28 & 0 & 210 \\
\hline WVREF627-211 & 27654 $\mid$ WVREF627 & 7/11/08 10:29 & 0 & 211 \\
\hline WVREF627-212 & \begin{tabular}{l|l|l}
27665 & WVREF627
\end{tabular} & 7/11/08 10:40 & 0.0003 & 212 \\
\hline WVREF627-213 & \begin{tabular}{l|l|}
27666 & WVREF627
\end{tabular} & 7/11/08 10:40 & 0 & 213 \\
\hline WVREF627-214 & \begin{tabular}{l|l}
27677 & WVREF627
\end{tabular} & 7/11/08 10:50 & -0.001 & 214 \\
\hline WVREF627-215 & \begin{tabular}{l|l|l|}
27678 & WVREF627
\end{tabular} & 7/11/08 10:50 & 0 & 215 \\
\hline WVREF627-216 & 27689|WVREF627 & 7/11/08 11:00 & 0.0008 & 216 \\
\hline WVREF627-217 & \begin{tabular}{l|l|l}
27719 & WVREF627
\end{tabular} & 7/11/08 18:18 & -0.0024 & 217 \\
\hline WVREF627-218 & \begin{tabular}{l|l}
27720 & WVREF627 \\
\end{tabular} & 7/11/08 18:18 & 0 & 218 \\
\hline WVREF627-219 & \begin{tabular}{l|l|l|}
27725 & WVREF627
\end{tabular} & 7/11/08 18:25 & -0.0018 & 219 \\
\hline WVREF627-220 & \begin{tabular}{l|l}
27726 & WVREF627 \\
\end{tabular} & $7 / 11 / 08$ 18:25 & 0 & 220 \\
\hline WVREF627-221 & \begin{tabular}{l|l|}
27737 & WVREF627 \\
\end{tabular} & 7/11/08 18:38 & 0.0006 & 221 \\
\hline WVREF627-222 & \begin{tabular}{l|l}
27738 & WVREF627
\end{tabular} & 7/11/08 18:38 & 0 & 222 \\
\hline WVREF627-223 & 27749 WVREF627 & 7/11/08 18:48 & 0.0027 & 223 \\
\hline WVREF627-224 & \begin{tabular}{l|l|l}
27759 & WVREF627 \\
\end{tabular} & $7 / 12 / 08$ 12:56 & 0 & 224 \\
\hline WVREF627-225 & \begin{tabular}{l|l}
27761 & WVREF627
\end{tabular} & 7/12/08 12:57| & -0.0001 & 225 \\
\hline WVREF627-226 & \begin{tabular}{l|l|}
27762 & WVREF627
\end{tabular} & 7/12/08 12:57 & 0 & 226 \\
\hline WVREF627-227 & \begin{tabular}{l|l}
27767 & WVREF627
\end{tabular} & 7/12/08 13:00 & -0.0028 & 227 \\
\hline WVREF627-228 & \begin{tabular}{l|l|}
27768 & WVREF627
\end{tabular} & 7/12/08 13:00 & 0 & 228 \\
\hline WVREF627-229 & \begin{tabular}{l|l|l|}
27779 & WVREF627 \\
\end{tabular} & 7/12/08 13:07 & -0.0001 & 229 \\
\hline WVREF627-230 & \begin{tabular}{|l|l|}
27780 & WVREF627
\end{tabular} & 7/12/08 13:09 & 0 & 230 \\
\hline WVREF627-231 & \begin{tabular}{|l|l|}
27791 & WVREF627 \\
\end{tabular} & 7/12/08 13:16 & 0.0003 & 231 \\
\hline WVREF627-232 & \begin{tabular}{l|l|}
27792 & WVREF627 \\
\end{tabular} & $7 / 13 / 08$ 14:55 & 0 & 232 \\
\hline WVREF627-233 & \begin{tabular}{l|l|}
27794 & WVREF627 \\
\end{tabular} & $7 / 13 / 08$ 14:56 & -0.0003 & 233 \\
\hline WVREF627-234 & \begin{tabular}{l|l|}
27795 & WVREF627 \\
\end{tabular} & $7 / 13 / 08$ 14:56 & 0 & 234 \\
\hline WVREF627-235 & \begin{tabular}{l|l|l}
27800 & WVREF627 \\
\end{tabular} & $7 / 13 / 08$ 14:59 & 0.0007 & 235 \\
\hline WVREF627-236 & \begin{tabular}{l|l}
27801 & WVREF627
\end{tabular} & $7 / 13 / 08$ 14:59 & 0 & 236 \\
\hline WVREF627-237 & \begin{tabular}{|l|l|}
27812 & WVREF627 \\
\end{tabular} & 7/13/08 15:07 & -0.0006 & 237 \\
\hline WVREF627-238 & \begin{tabular}{l|l|}
27813 & WVREF627 \\
\end{tabular} & 7/13/08 15:07 & 0 & 238 \\
\hline WVREF627-239 & \begin{tabular}{l|l|l}
27824 & WVREF627
\end{tabular} & $7 / 13 / 08$ 15:15 & 0.0038 & 239 \\
\hline WVREF627-240 & \begin{tabular}{l|l|}
27825 & WVREF627 \\
\end{tabular} & $7 / 14 / 08$ 11:56 & -0.0026 & 240 \\
\hline WVREF627-241 & \begin{tabular}{l|l|}
27826 & WVREF627
\end{tabular} & 7/14/08 12:01 & -0.0001 & 241 \\
\hline WVREF627-242 & \begin{tabular}{l|l}
27827 & WVREF627
\end{tabular} & $7 / 14 / 08$ 12:05 & -0.0001 & 242 \\
\hline WVREF627-243 & \begin{tabular}{l|l|}
27829 & WVREF627
\end{tabular} & 7/14/08 12:07 & 0.0002 & 243 \\
\hline WVREF627-244 & \begin{tabular}{l|l|l}
27830 & WVREF627
\end{tabular} & 7/14/08 12:07 & 0 & 244 \\
\hline WVREF627-245 & \begin{tabular}{l|l|l|}
27835 & WVREF627 \\
\end{tabular} & 7/14/08 12:13 & -0.0007 & 245 \\
\hline WVREF627-246 & \begin{tabular}{|l|l|}
27836 & WVREF627 \\
\end{tabular} & $7 / 14 / 08$ 12:13 & 0 & 246 \\
\hline WVREF627-247 & \begin{tabular}{l|l|}
27847 & WVREF627 \\
\end{tabular} & 7/14/08 12:25 & -0.0013 & 247 \\
\hline
\end{tabular}




\begin{tabular}{|c|c|c|c|c|c|}
\hline WVREF627-248 & 27848 & WVREF627 & $7 / 14 / 08$ 12:26 & 0.0001 & 248 \\
\hline WVREF627-249 & 27859 & WVREF627 & $7 / 14 / 08$ 12:38 & -0.0001 & 249 \\
\hline WVREF627-250 & 27860 & WVREF627 & $7 / 14 / 08$ 12:38 & 0 & 250 \\
\hline WVREF627-251 & 27871 & WVREF627 & $7 / 14 / 08$ 13:07 & -0.0004 & 251 \\
\hline WVREF627-252 & 27872 & WVREF627 & $7 / 14 / 08$ 13:08 & 0 & 252 \\
\hline WVREF627-253 & 27883 & WVREF627 & $7 / 14 / 08$ 13:19 & -0.0015 & 253 \\
\hline WVREF627-254 & 27884 & WVREF627 & $7 / 14 / 08$ 13:20 & 0 & 254 \\
\hline WVREF627-255 & 27895 & WVREF627 & $7 / 14 / 08$ 13:35 & -0.0023 & 255 \\
\hline WVREF627-256 & 27896 & WVREF627 & $7 / 14 / 08$ 13:35 & 0 & 256 \\
\hline WVREF627-257 & 27907 & WVREF627 & $7 / 14 / 08$ 13:49 & 0.0021 & 257 \\
\hline WVREF627-258 & 27908 & WVREF627 & $7 / 14 / 08$ 13:49 & 0 & 258 \\
\hline WVREF627-259 & 27919 & WVREF627 & $7 / 14 / 08$ 14:01 & 0.0002 & 259 \\
\hline WVREF627-260 & 27920 & WVREF627 & $7 / 14 / 08$ 14:02 & 0 & 260 \\
\hline WVREF627-261 & 27931 & WVREF627 & $7 / 14 / 08$ 14:18 & -0.0006 & 261 \\
\hline WVREF627-262 & 27932 & WVREF627 & $7 / 14 / 08$ 14:19 & 0 & 262 \\
\hline WVREF627-263 & 27943 & WVREF627 & $7 / 14 / 08$ 14:31 & 0.0006 & 263 \\
\hline WVREF627-264 & 27944 & WVREF627 & $7 / 14 / 08$ 14:31 & 0 & 264 \\
\hline WVREF627-265 & 27955 & WVREF627 & $7 / 14 / 08$ 14:43 & 0.0021 & 265 \\
\hline WVREF627-266 & 27976 & WVREF627 & $7 / 15 / 08$ 13:21 & 0 & 266 \\
\hline WVREF627-267 & 27978 & WVREF627 & $7 / 15 / 08$ 13:22 & 0.0003 & 267 \\
\hline WVREF627-268 & 27979 & WVREF627 & $7 / 15 / 08$ 13:22 & 0 & 268 \\
\hline WVREF627-269 & 27984 & WVREF627 & $7 / 15 / 08$ 13:25 & 0.0001 & 269 \\
\hline WVREF627-270 & 27985 & WVREF627 & $7 / 15 / 08$ 13:25 & 0 & 270 \\
\hline WVREF627-271 & 27986 & WVREF627 & $7 / 15 / 08$ 13:26 & 0 & 271 \\
\hline WVREF627-272 & 27997 & WVREF627 & $7 / 15 / 08$ 13:34 & -0.0019 & 272 \\
\hline WVREF627-273 & 27998 & WVREF627 & $7 / 15 / 08$ 13:34 & 0 & 273 \\
\hline WVREF627-274 & 28009 & WVREF627 & $7 / 15 / 08$ 13:43 & 0.0008 & 274 \\
\hline WVREF627-275 & 28010 & WVREF627 & 7/15/08 19:08 & -0.0009 & 275 \\
\hline WVREF627-276 & 28011 & WVREF627 & $7 / 15 / 08$ 19:10 & 0 & 276 \\
\hline WVREF627-277 & 28016 & WVREF627 & 7/15/08 19:14 & -0.0007 & 277 \\
\hline WVREF627-278 & 28017 & WVREF627 & $7 / 15 / 08$ 19:16 & 0 & 278 \\
\hline WVREF627-279 & 28028 & WVREF627 & $7 / 15 / 08$ 19:24 & 0.0012 & 279 \\
\hline WVREF627-280 & 28029 & WVREF627 & $7 / 15 / 08$ 19:27 & 0 & 280 \\
\hline WVREF627-281 & 28040 & WVREF627 & $7 / 15 / 08$ 19:36 & 0.0008 & 281 \\
\hline WVREF627-282 & 28073 & WVREF627 & $7 / 16 / 08$ 19:13 & -0.0021 & 282 \\
\hline WVREF627-283 & 28074 & WVREF627 & $7 / 16 / 08$ 19:13 & 0 & 283 \\
\hline WVREF627-284 & 28079 & WVREF627 & 7/16/08 19:19 & -0.0013 & 284 \\
\hline WVREF627-285 & 28080 & WVREF627 & $7 / 16 / 08$ 19:19 & 0 & 285 \\
\hline WVREF627-286 & 28091 & WVREF627 & 7/16/08 19:32 & -0.0004 & 286 \\
\hline WVREF627-287 & 28092 & WVREF627 & $7 / 16 / 08$ 19:32 & 0 & 287 \\
\hline WVREF627-288 & 28103 & WVREF627 & 7/16/08 19:44 & 0.0003 & 288 \\
\hline WVREF627-289 & 28104 & WVREF627 & $7 / 17 / 0814: 27$ & -0.0019 & 289 \\
\hline WVREF627-290 & 28105 & WVREF627 & $7 / 17 / 08$ 14:32 & 0 & 290 \\
\hline WVREF627-291 & 28107 & WVREF627 & $7 / 17 / 08$ 14:33 & -0.0004 & 291 \\
\hline WVREF627-292 & 28108 & WVREF627 & $7 / 17 / 08$ 14:34 & 0 & 292 \\
\hline WVREF627-293 & 28113 & WVREF627 & $7 / 17 / 08$ 14:38 & -0.0004 & 293 \\
\hline WVREF627-294 & 28114 & WVREF627 & $7 / 17 / 0814: 39$ & 0 & 294 \\
\hline WVREF627-295 & 28125 & WVREF627 & $7 / 17 / 08$ 14:48 & -0.0003 & 295 \\
\hline WVREF627-296 & 28126 & WVREF627 & $7 / 17 / 08$ 14:49 & 0 & 296 \\
\hline
\end{tabular}




\begin{tabular}{|c|c|c|c|c|}
\hline WVREF627-297 & \begin{tabular}{|l|l|}
28137 & WVREF627 \\
\end{tabular} & 7/17/08 14:58 & 0.0014 & 297 \\
\hline WVREF627-298 & \begin{tabular}{l|l}
28149 & WVREF627
\end{tabular} & 7/18/08 13:11 & 0.0001 & 298 \\
\hline WVREF627-299 & \begin{tabular}{l|l|l}
28150 & WVREF627
\end{tabular} & $7 / 18 / 08$ 13:11 & 0 & 299 \\
\hline WVREF627-300 & \begin{tabular}{l|l|l}
28155 & WVREF627
\end{tabular} & 7/18/08 13:18 & -0.0049 & 300 \\
\hline WVREF627-301 & \begin{tabular}{l|l|l}
28156 & WVREF627
\end{tabular} & 7/18/08 13:18 & 0 & 301 \\
\hline WVREF627-302 & \begin{tabular}{l|l|l}
28167 & WVREF627
\end{tabular} & 7/18/08 13:30 & 0 & 302 \\
\hline WVREF627-303 & \begin{tabular}{l|l|l|}
28178 & WVREF627
\end{tabular} & 7/18/08 13:43 & -0.0026 & 303 \\
\hline WVREF627-304 & \begin{tabular}{l|l|l}
28179 & WVREF627
\end{tabular} & 7/18/08 13:43 & 0 & 304 \\
\hline WVREF627-305 & \begin{tabular}{|l|l|}
28190 & WVREF627 \\
\end{tabular} & 7/18/08 13:56 & -0.0007 & 305 \\
\hline WVREF627-306 & \begin{tabular}{l|l}
28191 & WVREF627
\end{tabular} & 7/18/08 13:57| & 0 & 306 \\
\hline WVREF627-307 & \begin{tabular}{|l|l|}
28202 & WVREF627
\end{tabular} & 7/18/08 14:08 & 0.0003 & 307 \\
\hline WVREF627-308 & \begin{tabular}{|l|l|}
28203 & WVREF627
\end{tabular} & 7/18/08 14:08 & 0 & 308 \\
\hline WVREF627-309 & 28214 $\mid$ WVREF627 & $7 / 18 / 08$ 14:24 & 0.0002 & 309 \\
\hline WVREF627-310 & \begin{tabular}{l|l|l}
28215 & WVREF627
\end{tabular} & $7 / 18 / 08$ 14:24 & 0 & 310 \\
\hline WVREF627-311 & \begin{tabular}{l|l|}
28226 & WVREF627
\end{tabular} & $7 / 18 / 08$ 14:41 & 0.0015 & 311 \\
\hline WVREF627-312 & \begin{tabular}{l|l}
28227 & WVREF627
\end{tabular} & 7/18/08 14:41 & 0 & 312 \\
\hline WVREF627-313 & \begin{tabular}{|l|l|}
28238 & WVREF627
\end{tabular} & $7 / 18 / 08$ 14:56 & -0.0003 & 313 \\
\hline WVREF627-314 & 28239 WVREF627 & 7/18/08 14:57 & 0 & 314 \\
\hline WVREF627-315 & \begin{tabular}{l|l|l}
28250 & WVREF627
\end{tabular} & 7/18/08 15:10 & 0.0028 & 315 \\
\hline WVREF627-316 & \begin{tabular}{l|l}
28251 & WVREF627 \\
\end{tabular} & 7/18/08 15:19 & 0.0053 & 316 \\
\hline WVREF627-317 & \begin{tabular}{l|l}
28252 & WVREF627
\end{tabular} & 7/18/08 15:19 & 0 & 317 \\
\hline WVREF627-318 & \begin{tabular}{l|l}
28253 & WVREF627 \\
\end{tabular} & $7 / 18 / 08$ 15:22 & 0 & 318 \\
\hline WVREF627-319 & \begin{tabular}{l|l|}
28258 & WVREF627
\end{tabular} & $7 / 18 / 08$ 15:27 & -0.0021 & 319 \\
\hline WVREF627-320 & \begin{tabular}{l|l}
28259 & WVREF627
\end{tabular} & 7/18/08 15:28 & 0 & 320 \\
\hline WVREF627-321 & 28270 $\mid$ WVREF627 & 7/18/08 15:39 & 0.0004 & 321 \\
\hline WVREF627-322 & \begin{tabular}{l|l}
28271 & WVREF627
\end{tabular} & $7 / 18 / 08$ 15:39 & 0 & 322 \\
\hline WVREF627-323 & \begin{tabular}{l|l|}
28282 & WVREF627
\end{tabular} & 7/18/08 15:49 & 0.0009 & 323 \\
\hline WVREF627-324 & \begin{tabular}{|l|l|}
28283 & WVREF627
\end{tabular} & $7 / 18 / 08$ 15:50 & 0 & 324 \\
\hline WVREF627-325 & 28294|WVREF627 & 7/18/08 16:02 & 0.0006 & 325 \\
\hline WVREF627-326 & \begin{tabular}{l|l|l}
28295 & WVREF627
\end{tabular} & 7/18/08 16:06 & 0 & 326 \\
\hline WVREF627-327 & \begin{tabular}{|l|l|}
28306 & WVREF627 \\
\end{tabular} & 7/18/08 16:16 & 0.0005 & 327 \\
\hline WVREF627-328 & \begin{tabular}{|l|l|}
28307 & WVREF627
\end{tabular} & $7 / 18 / 08$ 16:16 & 0 & 328 \\
\hline WVREF627-329 & \begin{tabular}{|l|l|}
28318 & WVREF627 \\
\end{tabular} & 7/18/08 16:28 & -0.0014 & 329 \\
\hline WVREF627-330 & \begin{tabular}{l|l}
28319 & WVREF627 \\
\end{tabular} & 7/18/08 16:28 & 0 & 330 \\
\hline WVREF627-331 & \begin{tabular}{l|l}
28330 & WVREF627 \\
\end{tabular} & 7/18/08 16:37 & 0.0026 & 331 \\
\hline WVREF627-332 & \begin{tabular}{l|l}
28331 & WVREF627 \\
\end{tabular} & 7/18/08 16:42 & 0 & 332 \\
\hline WVREF627-333 & \begin{tabular}{|l|l|}
28342 & WVREF627 \\
\end{tabular} & $7 / 18 / 08$ 16:53 & 0.0017 & 333 \\
\hline WVREF627-334 & \begin{tabular}{l|l|}
28343 & WVREF627
\end{tabular} & 7/18/08 16:53 & 0 & 334 \\
\hline WVREF627-335 & \begin{tabular}{|l|l|}
28346 & WVREF627 \\
\end{tabular} & $7 / 18 / 08$ 16:54 & 0.0006 & 335 \\
\hline WVREF627-336 & 28384 WVREF627 & $7 / 21 / 08$ 11:08 & -0.0013 & 336 \\
\hline WVREF627-337 & \begin{tabular}{l|l|l}
28385 & WVREF627
\end{tabular} & $7 / 21 / 08$ 11:10 & 0 & 337 \\
\hline WVREF627-338 & \begin{tabular}{l|l}
28390 & WVREF627 \\
\end{tabular} & 7/21/08 11:14 & -0.0005 & 338 \\
\hline WVREF627-339 & \begin{tabular}{l|l|}
28391 & WVREF627
\end{tabular} & $7 / 21 / 08$ 11:18 & 0 & 339 \\
\hline WVREF627-340 & \begin{tabular}{|l|l|}
28402 & WVREF627 \\
\end{tabular} & $7 / 21 / 08$ 11:25 & -0.0005 & 340 \\
\hline WVREF627-341 & \begin{tabular}{l|l|}
28403 & WVREF627
\end{tabular} & $7 / 21 / 08$ 11:27 & 0 & 341 \\
\hline WVREF627-342 & 28414 WVREF627 & 7/21/08 11:35 & 0 & 342 \\
\hline WVREF627-343 & \begin{tabular}{l|l|l|}
28415 & WVREF627 \\
\end{tabular} & 7/21/08 11:39 & 0 & 343 \\
\hline WVREF627-344 & \begin{tabular}{|l|l|}
28426 & WVREF627 \\
\end{tabular} & $7 / 21 / 08$ 11:48 & 0.0006 & 344 \\
\hline WVREF627-345 & \begin{tabular}{l|l|l|}
28427 & WVREF627 \\
\end{tabular} & 7/21/08 11:52 & 0 & 345 \\
\hline
\end{tabular}




\begin{tabular}{|c|c|c|c|c|}
\hline WVREF627-346 & \begin{tabular}{l|l|l|}
28438 & WVREF627 \\
\end{tabular} & 7/21/08 12:01 & 0.0009 & 346 \\
\hline WVREF627-347 & 28439 WVREF627 & $7 / 21 / 08$ 12:03 & 0 & 347 \\
\hline WVREF627-348 & 28450|WVREF627 & $7 / 21 / 08$ 12:11 & 0.0019 & 348 \\
\hline WVREF627-349 & \begin{tabular}{l|l|}
28451 & WVREF627
\end{tabular} & 7/21/08 12:13 & 0 & 349 \\
\hline WVREF627-350 & \begin{tabular}{l|l|l}
28462 & WVREF627
\end{tabular} & $7 / 21 / 08$ 12:23 & 0.0002 & 350 \\
\hline WVREF627-351 & \begin{tabular}{l|l}
28463 & WVREF627
\end{tabular} & $7 / 21 / 08$ 12:24 & 0 & 351 \\
\hline WVREF627-352 & 28474 $\mid$ WVREF627 & $7 / 21 / 08$ 12:33 & 0.0029 & 352 \\
\hline WVREF627-353 & 28475|WVREF627 & $7 / 21 / 08$ 12:34 & 0.0027 & 353 \\
\hline WVREF627-354 & \begin{tabular}{l|l|l}
28476 & WVREF627 \\
\end{tabular} & $7 / 21 / 08$ 12:36 & 0 & 354 \\
\hline WVREF627-355 & $28479 \mid$ WVREF627 & $7 / 21 / 08$ 12:38 & -0.0006 & 355 \\
\hline WVREF627-356 & \begin{tabular}{|l|l|}
28480 & WVREF627 \\
\end{tabular} & $7 / 21 / 08$ 12:39 & -0.001 & 356 \\
\hline WVREF627-357 & \begin{tabular}{l|l|}
28490 & WVREF627
\end{tabular} & $7 / 22 / 08$ 12:05 & -0.0025 & 357 \\
\hline WVREF627-358 & 28490 WVREF627 & $7 / 22 / 08$ 12:05 & -0.0025 & 358 \\
\hline WVREF627-359 & 28491 $\mid$ WVREF627 & $7 / 22 / 08$ 12:06 & 0 & 359 \\
\hline WVREF627-360 & \begin{tabular}{l|l|}
28491 & WVREF627 \\
\end{tabular} & $7 / 22 / 08$ 12:06 & 0 & 360 \\
\hline WVREF627-361 & 28496 $\mid$ WVREF627 & $7 / 22 / 08$ 12:12 & -0.0013 & 361 \\
\hline WVREF627-362 & \begin{tabular}{l|l|l} 
28496 & WVREF627 \\
\end{tabular} & $7 / 22 / 08$ 12:12 & -0.0013 & 362 \\
\hline WVREF627-363 & \begin{tabular}{l|l|l}
28497 & WVREF627
\end{tabular} & $7 / 22 / 08$ 12:12 & 0 & 363 \\
\hline WVREF627-364 & \begin{tabular}{l|l|l}
28497 & WVREF627 \\
\end{tabular} & $7 / 22 / 08$ 12:12 & 0 & 364 \\
\hline WVREF627-365 & 28508|WVREF627 & $7 / 22 / 08$ 12:24 & -0.0004 & 365 \\
\hline WVREF627-366 & \begin{tabular}{l|l|l}
28508 & WVREF627
\end{tabular} & $7 / 22 / 08$ 12:24 & -0.0004 & 366 \\
\hline WVREF627-367 & \begin{tabular}{l|l|l|}
28509 & WVREF627 \\
\end{tabular} & $7 / 22 / 08$ 12:25 & 0 & 367 \\
\hline WVREF627-368 & 28509 WVREF627 & $7 / 22 / 08$ 12:25 & 0 & 368 \\
\hline WVREF627-369 & 28520 $\mid$ WVREF627 & $7 / 22 / 08$ 12:37 & 0.0012 & 369 \\
\hline WVREF627-370 & \begin{tabular}{l|l|l}
28520 & WVREF627 \\
\end{tabular} & $7 / 22 / 08$ 12:37 & 0.0012 & 370 \\
\hline WVREF627-371 & \begin{tabular}{l|l|}
28521 & WVREF627 \\
\end{tabular} & $7 / 22 / 08$ 12:37 & 0 & 371 \\
\hline WVREF627-372 & \begin{tabular}{l|l|}
28521 & WVREF627 \\
\end{tabular} & 7/22/08 12:37 & 0 & 372 \\
\hline WVREF627-373 & 28532 $\mid$ WVREF627 & $7 / 22 / 08$ 12:49 & -0.001 & 373 \\
\hline WVREF627-374 & \begin{tabular}{l|l|l}
28532 & WVREF627 \\
\end{tabular} & $7 / 22 / 08$ 12:49 & -0.001 & 374 \\
\hline WVREF627-375 & 28533|WVREF627 & $7 / 22 / 08$ 12:50 & 0 & 375 \\
\hline WVREF627-376 & \begin{tabular}{l|l|}
28533 & WVREF627
\end{tabular} & $7 / 22 / 08$ 12:50 & 0 & 376 \\
\hline WVREF627-377 & $28544 \mid$ WVREF627 & 7/22/08 13:07 & 0.0008 & 377 \\
\hline WVREF627-378 & \begin{tabular}{l|l|}
28544 & WVREF627 \\
\end{tabular} & 7/22/08 13:07 & 0.0008 & 378 \\
\hline WVREF627-379 & \begin{tabular}{l|l|}
28545 & WVREF627
\end{tabular} & $7 / 22 / 08$ 13:07 & 0 & 379 \\
\hline WVREF627-380 & 28545 WVREF627 & 7/22/08 13:07 & 0 & 380 \\
\hline WVREF627-381 & \begin{tabular}{l|l|}
28556 & WVREF627 \\
\end{tabular} & $7 / 22 / 08$ 13:19 & -0.0001 & 381 \\
\hline WVREF627-382 & \begin{tabular}{|l|l|}
28556 & WVREF627 \\
\end{tabular} & 7/22/08 13:19 & -0.0001 & 382 \\
\hline WVREF627-383 & 28557 WVREF627 & $7 / 22 / 08$ 13:19 & 0 & 383 \\
\hline WVREF627-384 & 28557 WVREF627 & 7/22/08 13:19 & 0 & 384 \\
\hline WVREF627-385 & 28560 WVREF627 & 7/22/08 13:22 & -0.0002 & 385 \\
\hline WVREF627-386 & \begin{tabular}{l|l|}
28560 & WVREF627 \\
\end{tabular} & 7/22/08 13:22 & -0.0002 & 386 \\
\hline WVREF627-387 & \begin{tabular}{l|l|l|}
28561 & WVREF627 \\
\end{tabular} & $7 / 23 / 08$ 11:20 & -0.005 & 387 \\
\hline WVREF627-388 & \begin{tabular}{l|l|}
28562 & WVREF627 \\
\end{tabular} & $7 / 23 / 08$ 11:24 & 0 & 388 \\
\hline WVREF627-389 & 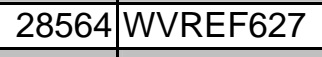 & $7 / 23 / 08$ 11:26 & -0.0004 & 389 \\
\hline WVREF627-390 & 28565 WVREF627 & $7 / 23 / 08$ 11:26 & 0 & 390 \\
\hline WVREF627-391 & \begin{tabular}{|l|l|}
28570 & WVREF627 \\
\end{tabular} & 7/23/08 11:29 & 0.0009 & 391 \\
\hline WVREF627-392 & \begin{tabular}{l|l|l|}
28571 & WVREF627 \\
\end{tabular} & 7/23/08 11:31 & 0 & 392 \\
\hline WVREF627-393 & \begin{tabular}{l|l|l}
28582 & WVREF627 \\
\end{tabular} & 7/23/08 11:38 & 0.0001 & 393 \\
\hline WVREF627-394 & \begin{tabular}{l|l|}
28583 & WVREF627 \\
\end{tabular} & $7 / 23 / 08$ 11:40 & 0 & 394 \\
\hline
\end{tabular}




\begin{tabular}{|c|c|c|c|c|}
\hline WVREF627-395 & \begin{tabular}{l|l|l|}
28594 & WVREF627 \\
\end{tabular} & 7/23/08 11:48 & 0.0006 & 395 \\
\hline WVREF627-396 & $28595 \mid$ WVREF627 & $7 / 23 / 08$ 11:49 & 0 & 396 \\
\hline WVREF627-397 & $\begin{array}{l}\text { 28606|WVREF627 } \\
\end{array}$ & 7/23/08 11:57 & -0.0001 & 397 \\
\hline WVREF627-398 & \begin{tabular}{l|l}
28607 & WVREF627 \\
\end{tabular} & $7 / 23 / 08$ 11:58 & 0 & 398 \\
\hline WVREF627-399 & \begin{tabular}{|l|l|} 
28618 & WVEF627
\end{tabular} & $7 / 23 / 08$ 12:05 & 0.0009 & 399 \\
\hline WVREF627-400 & 28619 WVREF627 & $7 / 23 / 08$ 12:07 & 0 & 400 \\
\hline WVREF627-401 & 28630|WVREF627 & $7 / 23 / 08$ 12:16 & -0.0005 & 401 \\
\hline WVREF627-402 & \begin{tabular}{l|l|}
28631 & WVREF627 \\
\end{tabular} & $7 / 23 / 08$ 12:16 & 0 & 402 \\
\hline WVREF627-403 & 28634 $\mid$ WVREF627 & $7 / 23 / 08$ 12:18 & 0.0003 & 403 \\
\hline WVREF627-404 & 28635 WVREF627 & $7 / 24 / 08$ 12:05 & 0 & 404 \\
\hline WVREF627-405 & \begin{tabular}{|l|l|}
28636 & WVREF627 \\
\end{tabular} & 7/24/08 12:11 & 0 & 405 \\
\hline WVREF627-406 & 28638 $\mid$ WVREF627 & $7 / 24 / 08$ 12:13 & 0.0003 & 406 \\
\hline WVREF627-407 & 28639 WVREF627 & $7 / 24 / 08$ 12:13 & 0 & 407 \\
\hline WVREF627-408 & \begin{tabular}{l|l|}
28644 & WVREF627
\end{tabular} & $7 / 24 / 08$ 12:16 & 0.0007 & 408 \\
\hline WVREF627-409 & \begin{tabular}{l|l|l|}
28645 & WVREF627 \\
\end{tabular} & $7 / 24 / 08$ 12:18 & 0 & 409 \\
\hline WVREF627-410 & \begin{tabular}{|l|l|}
28656 & WVREF627 \\
\end{tabular} & $7 / 24 / 08$ 12:25 & 0 & 410 \\
\hline WVREF627-411 & 28663|WVREF627 & $7 / 24 / 08$ 12:32 & 0.0017 & 411 \\
\hline WVREF627-412 & \begin{tabular}{l|l}
28681 & WVREF627 \\
\end{tabular} & 7/24/08 18:03 & -0.001 & 412 \\
\hline WVREF627-413 & \begin{tabular}{|l|l|}
28682 & WVREF627 \\
\end{tabular} & 7/24/08 18:04 & 0 & 413 \\
\hline WVREF627-414 & \begin{tabular}{l|l|l|}
28687 & WVREF627 \\
\end{tabular} & 7/24/08 18:08 & -0.0011 & 414 \\
\hline WVREF627-415 & \begin{tabular}{|l|l|}
28688 & WVREF627 \\
\end{tabular} & $7 / 24 / 08$ 18:09 & -0.0015 & 415 \\
\hline WVREF627-416 & \begin{tabular}{l|l}
28689 & WVREF627 \\
\end{tabular} & 7/24/08 18:09 & 0 & 416 \\
\hline WVREF627-417 & 28700 $\mid$ WVREF627 & $7 / 24 / 08$ 18:17 & -0.0004 & 417 \\
\hline WVREF627-418 & 28701 WVREF627 & 7/24/08 18:18 & 0 & 418 \\
\hline WVREF627-419 & 28708|WVREF627 & $7 / 24 / 08$ 18:22 & 0.0002 & 419 \\
\hline WVREF627-420 & \begin{tabular}{|l|l|}
28710 & WVREF627 \\
\end{tabular} & 7/25/08 13:01 & 0 & 420 \\
\hline WVREF627-421 & \begin{tabular}{l|l|l}
28712 & WVREF627
\end{tabular} & $7 / 25 / 08$ 13:02 & 0.0001 & 421 \\
\hline WVREF627-422 & 28713 $\mid$ WVREF627 & 7/25/08 13:02 & 0 & 422 \\
\hline WVREF627-423 & 28718|WVREF627 & $7 / 25 / 08$ 13:05 & -0.0001 & 423 \\
\hline WVREF627-424 & \begin{tabular}{|l|l|}
28719 & WVREF627 \\
\end{tabular} & 7/25/08 13:07 & 0 & 424 \\
\hline WVREF627-425 & 28730|WVREF627 & $7 / 25 / 08$ 13:15 & -0.0025 & 425 \\
\hline WVREF627-426 & 28731 $\mid$ WVREF627 & $7 / 25 / 08$ 13:16 & 0 & 426 \\
\hline WVREF627-427 & 28738|WVREF627 & $7 / 25 / 08$ 13:21 & -0.0011 & 427 \\
\hline WVREF627-428 & \begin{tabular}{l|l|l}
28739 & WVREF627 \\
\end{tabular} & $7 / 25 / 08$ 13:22 & 0 & 428 \\
\hline WVREF627-429 & 28750|WVREF627 & 7/25/08 13:30 & -0.0015 & 429 \\
\hline WVREF627-430 & 28751 WVREF627 & $7 / 25 / 08$ 13:30 & 0 & 430 \\
\hline WVREF627-431 & \begin{tabular}{l|l|l|}
28762 & WVREF627 \\
\end{tabular} & 7/25/08 13:38 & -0.0012 & 431 \\
\hline WVREF627-432 & \begin{tabular}{l|l|l|}
28763 & WVREF627 \\
\end{tabular} & $7 / 25 / 08$ 13:39 & 0 & 432 \\
\hline WVREF627-433 & $28774 \mid$ WVREF627 & 7/25/08 13:46 & -0.0025 & 433 \\
\hline WVREF627-434 & 28775 WVREF627 & 7/25/08 13:48 & 0 & 434 \\
\hline WVREF627-435 & \begin{tabular}{l|l|l}
28776 & WVREF627 \\
\end{tabular} & 7/25/08 15:06 & -0.0084 & 435 \\
\hline WVREF627-436 & 28777 WVREF627 & 7/25/08 15:07 & 0 & 436 \\
\hline WVREF627-437 & \begin{tabular}{|l|l|}
28782 & WVREF627 \\
\end{tabular} & $7 / 25 / 08$ 15:11 & 0.0012 & 437 \\
\hline WVREF627-438 & 28783 WVREF627 & $7 / 25 / 08$ 15:12 & 0 & 438 \\
\hline WVREF627-439 & 28784|WVREF627 & $7 / 25 / 08$ 15:13 & 0 & 439 \\
\hline WVREF627-440 & 28795 WVREF627 & $7 / 25 / 08$ 15:28 & -0.0013 & 440 \\
\hline WVREF627-441 & \begin{tabular}{|l|l|}
28796 & WVREF627 \\
\end{tabular} & $7 / 25 / 08$ 15:29 & 0 & 441 \\
\hline WVREF627-442 & 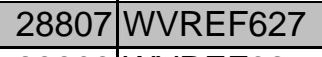 & $7 / 25 / 08$ 15:40 & -0.0006 & 442 \\
\hline WVREF627-443 & \begin{tabular}{l|l|l} 
28808 & WVREF627 \\
\end{tabular} & 7/25/08 15:41 & 0 & 443 \\
\hline
\end{tabular}




\begin{tabular}{|c|c|c|c|c|}
\hline WVREF627-444 & 28819 WVREF627 & 7/25/08 15:52 & -0.0009 & 444 \\
\hline WVREF627-445 & \begin{tabular}{l|l|} 
28820 & WVREF627
\end{tabular} & $7 / 25 / 08$ 15:52 & 0 & 445 \\
\hline WVREF627-446 & \begin{tabular}{|l|l|} 
28831|WVREF27 \\
\end{tabular} & $7 / 25 / 08$ 16:04 & -0.0008 & 446 \\
\hline WVREF627-447 & \begin{tabular}{l|l}
28832 & WVREF627 \\
\end{tabular} & $7 / 25 / 08$ 16:05 & 0 & 447 \\
\hline WVREF627-448 & \begin{tabular}{l|l|}
28843 & WVREF627
\end{tabular} & $7 / 25 / 08$ 16:17 & -0.0007 & 448 \\
\hline WVREF627-449 & 28844 $\mid$ WVREF627 & $7 / 25 / 08$ 16:18 & 0 & 449 \\
\hline WVREF627-450 & $28855 \mid$ WVREF627 & 7/25/08 16:31 & 0 & 450 \\
\hline WVREF627-451 & \begin{tabular}{l|l|}
28856 & WVREF627
\end{tabular} & $7 / 25 / 08$ 16:31 & 0 & 451 \\
\hline WVREF627-452 & \begin{tabular}{l|l|l|}
28867 & WVREF627 \\
\end{tabular} & $7 / 25 / 08$ 16:44 & 0 & 452 \\
\hline WVREF627-453 & \begin{tabular}{l|l}
28898 & WVREF627
\end{tabular} & 7/26/08 14:53 & 0 & 453 \\
\hline WVREF627-454 & \begin{tabular}{l|l|}
28900 & WVREF627 \\
\end{tabular} & $7 / 26 / 08$ 14:54 & -0.0001 & 454 \\
\hline WVREF627-455 & \begin{tabular}{l|l|}
28901 & WVREF627
\end{tabular} & 7/26/08 14:54 & 0 & 455 \\
\hline WVREF627-456 & \begin{tabular}{l|l|l}
28906 & WVREF627 \\
\end{tabular} & $7 / 26 / 08$ 14:57 & -0.0003 & 456 \\
\hline WVREF627-457 & 28907 WVREF627 & $7 / 26 / 08$ 14:57 & 0 & 457 \\
\hline WVREF627-458 & \begin{tabular}{l|l|}
28916 & WVREF627 \\
\end{tabular} & $7 / 26 / 08$ 15:04 & -0.0007 & 458 \\
\hline WVREF627-459 & \begin{tabular}{l|l|}
28917 & WVREF627
\end{tabular} & $7 / 26 / 08$ 15:04 & 0 & 459 \\
\hline WVREF627-460 & \begin{tabular}{l|l|l}
28926 & WVREF627 \\
\end{tabular} & $7 / 26 / 08$ 15:11 & -0.0019 & 460 \\
\hline WVREF627-461 & \begin{tabular}{l|l|}
28927 & WVREF627
\end{tabular} & $7 / 26 / 08$ 15:23 & 0 & 461 \\
\hline WVREF627-462 & \begin{tabular}{l|l|}
28938 & WVREF627 \\
\end{tabular} & $7 / 26 / 08$ 15:32 & 0.0005 & 462 \\
\hline WVREF627-463 & 28939|WVREF627 & $7 / 26 / 08$ 15:32 & 0 & 463 \\
\hline WVREF627-464 & 28950|WVREF627 & 7/26/08 15:41 & -0.0011 & 464 \\
\hline WVREF627-465 & \begin{tabular}{l|l|}
28951 & WVREF627 \\
\end{tabular} & 7/26/08 15:41 & 0 & 465 \\
\hline WVREF627-466 & 28961|WVREF627 & 7/26/08 15:49 & -0.0015 & 466 \\
\hline WVREF627-467 & \begin{tabular}{l|l|}
28962 & WVREF627 \\
\end{tabular} & $7 / 27 / 08$ 14:05 & -0.0003 & 467 \\
\hline WVREF627-468 & \begin{tabular}{l|l}
28963 & WVREF627
\end{tabular} & 7/27/08 14:09 & 0 & 468 \\
\hline WVREF627-469 & \begin{tabular}{l|l|l|}
28965 & WVREF627
\end{tabular} & $7 / 27 / 08$ 14:11 & 0.0022 & 469 \\
\hline WVREF627-470 & \begin{tabular}{l|l|}
28966 & WVREF627 \\
\end{tabular} & $7 / 27 / 08$ 14:11 & 0 & 470 \\
\hline WVREF627-471 & 28968|WVREF627 & $7 / 27 / 08$ 14:12 & -0.0005 & 471 \\
\hline WVREF627-472 & 28969 WVREF627 & $7 / 27 / 08$ 14:12 & 0 & 472 \\
\hline WVREF627-473 & 28974|WVREF627 & 7/27/08 14:16 & -0.0023 & 473 \\
\hline WVREF627-474 & \begin{tabular}{l|l|l}
28975 & WVREF627
\end{tabular} & $7 / 27 / 08$ 14:17 & 0 & 474 \\
\hline WVREF627-475 & 28985|WVREF627 & $7 / 27 / 08$ 14:24 & -0.0015 & 475 \\
\hline WVREF627-476 & \begin{tabular}{|l|l|}
28986 & WVREF627 \\
\end{tabular} & $7 / 27 / 0814: 25$ & 0 & 476 \\
\hline WVREF627-477 & \begin{tabular}{l|l|}
28997 & WVREF627
\end{tabular} & $7 / 27 / 08$ 14:32 & 0 & 477 \\
\hline WVREF627-478 & \begin{tabular}{|l|l|}
29008 & WVREF627 \\
\end{tabular} & $7 / 27 / 08$ 14:41 & -0.0007 & 478 \\
\hline WVREF627-479 & $29019 \mid$ WVREF627 & 7/28/08 18:05 & 0.0081 & 479 \\
\hline WVREF627-480 & \begin{tabular}{|l|l|}
29020 & WVREF627 \\
\end{tabular} & 7/28/08 18:09 & 0 & 480 \\
\hline WVREF627-481 & \begin{tabular}{l|l|}
29022 & WVREF627
\end{tabular} & 7/28/08 18:11 & 0.0001 & 481 \\
\hline WVREF627-482 & 29023 WVREF627 & $7 / 28 / 08$ 18:11 & 0 & 482 \\
\hline WVREF627-483 & \begin{tabular}{l|l|}
29028 & WVREF627 \\
\end{tabular} & $7 / 28 / 08$ 18:14 & -0.0024 & 483 \\
\hline WVREF627-484 & \begin{tabular}{l|l}
29029 & WVREF627 \\
\end{tabular} & $7 / 28 / 08$ 18:15 & 0 & 484 \\
\hline WVREF627-485 & 29039 WVREF627 & $7 / 28 / 08$ 18:22 & -0.0009 & 485 \\
\hline WVREF627-486 & 29040|WVREF627 & $7 / 28 / 08$ 18:23 & 0 & 486 \\
\hline WVREF627-487 & \begin{tabular}{l|l|l|}
29051 & WVREF627 \\
\end{tabular} & 7/28/08 18:31 & -0.0015 & 487 \\
\hline WVREF627-488 & $29052 \mid$ WVREF627 & 7/28/08 18:31 & 0 & 488 \\
\hline WVREF627-489 & $29063 \mid$ WVREF627 & 7/28/08 18:38 & -0.0008 & 489 \\
\hline WVREF627-490 & 29084|WVREF627 & 7/29/08 12:07 & -0.0007 & 490 \\
\hline WVREF627-491 & \begin{tabular}{l|l|l|}
29085 & WVREF627 \\
\end{tabular} & 7/29/08 12:09 & 0 & 491 \\
\hline WVREF627-492 & \begin{tabular}{l|l|}
29090 & WVREF627 \\
\end{tabular} & 7/29/08 12:13 & -0.0048 & 492 \\
\hline
\end{tabular}




\begin{tabular}{|c|c|c|c|c|}
\hline WVREF627-493 & 29091|WVREF627 & 7/29/08 12:13 & 0 & 493 \\
\hline WVREF627-494 & \begin{tabular}{l|l|} 
29096 & WVREF627
\end{tabular} & $7 / 29 / 08$ 12:16 & -0.0014 & 494 \\
\hline WVREF627-495 & \begin{tabular}{l|l}
29097 & WVREF627
\end{tabular} & $7 / 29 / 08$ 12:18 & 0 & 495 \\
\hline WVREF627-496 & \begin{tabular}{l|l}
29107 & WVREF627
\end{tabular} & $7 / 29 / 08$ 12:24 & -0.0007 & 496 \\
\hline WVREF627-497 & 29108|WVREF627 & $7 / 29 / 08$ 12:25 & 0 & 497 \\
\hline WVREF627-498 & 29119|WVREF627 & 7/29/08 12:32 & -0.0005 & 498 \\
\hline WVREF627-499 & 29120|WVREF627 & $7 / 29 / 08$ 12:33 & 0 & 499 \\
\hline WVREF627-500 & \begin{tabular}{l|l}
29131 & WVREF627 \\
\end{tabular} & $7 / 29 / 08$ 12:40 & -0.001 & 500 \\
\hline WVREF627-501 & 29145|WVREF627 & 7/30/08 10:59 & -0.0006 & 501 \\
\hline WVREF627-502 & \begin{tabular}{l|l}
29146 & WVREF627 \\
\end{tabular} & 7/30/08 11:02 & 0 & 502 \\
\hline WVREF627-503 & 29151|WVREF627 & 7/30/08 11:05 & -0.0035 & 503 \\
\hline WVREF627-504 & \begin{tabular}{l|l|}
29152 & WVREF627
\end{tabular} & 7/30/08 11:05 & 0 & 504 \\
\hline WVREF627-505 & 29157 WVREF627 & 7/30/08 11:08 & -0.0004 & 505 \\
\hline WVREF627-506 & \begin{tabular}{|l|l|}
29158 & WVREF627
\end{tabular} & 7/30/08 11:09 & 0 & 506 \\
\hline WVREF627-507 & $29169 \mid$ WVREF627 & 7/30/08 11:18 & -0.0007 & 507 \\
\hline WVREF627-508 & 29170|WVREF627 & 7/30/08 11:19 & 0 & 508 \\
\hline WVREF627-509 & \begin{tabular}{|l|l|}
29181 & WVREF627 \\
\end{tabular} & 7/30/08 11:27 & 0.0004 & 509 \\
\hline WVREF627-510 & \begin{tabular}{l|l|}
29182 & WVREF627
\end{tabular} & 7/30/08 11:27 & 0 & 510 \\
\hline WVREF627-511 & 29193 WVREF627 & 7/30/08 11:36 & 0.0004 & 511 \\
\hline WVREF627-512 & 29194 WVREF627 & $7 / 30 / 08$ 11:36 & 0 & 512 \\
\hline WVREF627-513 & 29205|WVREF627 & $7 / 30 / 08$ 11:45 & -0.001 & 513 \\
\hline WVREF627-514 & \begin{tabular}{|l|l|}
29206 & WVREF627 \\
\end{tabular} & $7 / 30 / 08$ 11:45 & 0 & 514 \\
\hline WVREF627-515 & \begin{tabular}{l|l|l|}
29217 & WVREF627 \\
\end{tabular} & $7 / 30 / 08$ 11:55 & 0.0002 & 515 \\
\hline WVREF627-516 & \begin{tabular}{l|l}
29218 & WVREF627 \\
\end{tabular} & 7/30/08 11:57 & 0 & 516 \\
\hline WVREF627-517 & 29229 WVREF627 & $7 / 30 / 08$ 12:07 & 0.0004 & 517 \\
\hline WVREF627-518 & \begin{tabular}{|l|l|}
29230 & WVREF627 \\
\end{tabular} & $7 / 30 / 08$ 12:08 & 0 & 518 \\
\hline WVREF627-519 & 29241|WVREF627 & $7 / 30 / 08$ 12:17 & 0.0009 & 519 \\
\hline WVREF627-520 & \begin{tabular}{|l|l|}
29242 & WVREF627 \\
\end{tabular} & 7/30/08 12:17 & 0 & 520 \\
\hline WVREF627-521 & \begin{tabular}{l|l|}
29253 & WVREF627
\end{tabular} & $7 / 30 / 08$ 12:27 & -0.0002 & 521 \\
\hline WVREF627-522 & 29254 WVREF627 & 7/30/08 12:30 & 0 & 522 \\
\hline WVREF627-523 & 29265|WVREF627 & $7 / 30 / 08$ 12:38 & 0.0011 & 523 \\
\hline WVREF627-524 & \begin{tabular}{|l|l|}
29266 & WVREF627 \\
\end{tabular} & $7 / 30 / 08$ 12:39 & 0 & 524 \\
\hline WVREF627-525 & 29277 WVREF627 & $7 / 30 / 08$ 12:46 & -0.0002 & 525 \\
\hline WVREF627-526 & \begin{tabular}{l|l|}
29278 & WVREF627 \\
\end{tabular} & 7/31/08 15:15 & -0.0033 & 526 \\
\hline WVREF627-527 & 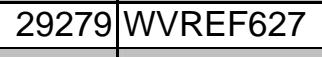 & 7/31/08 15:19 & 0 & 527 \\
\hline WVREF627-528 & 29281 WVREF627 & 7/31/08 15:21 & 0.0004 & 528 \\
\hline WVREF627-529 & \begin{tabular}{|l|l|}
29282 & WVREF627 \\
\end{tabular} & 7/31/08 15:21 & 0 & 529 \\
\hline WVREF627-530 & 29287 WVREF627 & 7/31/08 15:24 & 0.0017 & 530 \\
\hline WVREF627-531 & 29288 $\mid$ WVREF627 & 7/31/08 15:25 & 0 & 531 \\
\hline WVREF627-532 & 29295|WVREF627 & 7/31/08 15:29 & 0.0003 & 532 \\
\hline WVREF627-533 & \begin{tabular}{|l|l|}
29296 & WVREF627 \\
\end{tabular} & 7/31/08 15:29 & 0 & 533 \\
\hline WVREF627-534 & 29303 WVREF627 & $7 / 31 / 08$ 15:36 & -0.0002 & 534 \\
\hline WVREF627-535 & 29304 $\mid$ WVREF627 & 8/1/08 13:30 & 0.002 & 535 \\
\hline WVREF627-536 & 29305 WVREF627 & 8/1/08 13:36 & 0 & 536 \\
\hline WVREF627-537 & 29307 WVREF627 & 8/1/08 13:37 & 0.0004 & 537 \\
\hline WVREF627-538 & \begin{tabular}{|l|l|}
29308 & WVREF627 \\
\end{tabular} & 8/1/08 13:37 & 0 & 538 \\
\hline WVREF627-539 & 29313|WVREF627 & 8/1/08 13:40 & -0.0006 & 539 \\
\hline WVREF627-540 & 29314 WVREF627 & $8 / 1 / 08$ 13:43 & 0 & 540 \\
\hline WVREF627-541 & \begin{tabular}{l|l|l|}
29325 & WVREF627 \\
\end{tabular} & 8/1/08 13:51 & 0.001 & 541 \\
\hline
\end{tabular}




\begin{tabular}{|c|c|c|c|c|c|}
\hline WVREF627-542 & 29326 & WVREF627 & 8/1/08 13:53 & 0 & 542 \\
\hline WVREF627-543 & 29337 & WVREF627 & 8/1/08 14:03 & 0.0003 & 543 \\
\hline WVREF627-544 & 29338 & WVREF627 & 8/1/08 14:04 & 0 & 544 \\
\hline WVREF627-545 & 29341 & WVREF627 & $8 / 1 / 08$ 14:06 & 0.0008 & 545 \\
\hline WVREF627-546 & 29366 & WVREF627 & $8 / 2 / 08$ 14:14 & -0.0005 & 546 \\
\hline WVREF627-547 & 29367 & WVREF627 & $8 / 2 / 0814: 18$ & 0 & 547 \\
\hline WVREF627-548 & 29369 & WVREF627 & $8 / 2 / 08$ 14:19 & 0.0004 & 548 \\
\hline WVREF627-549 & 29370 & WVREF627 & $8 / 2 / 0814: 20$ & 0 & 549 \\
\hline WVREF627-550 & 29375 & WVREF627 & $8 / 2 / 08$ 14:24 & 0.0011 & 550 \\
\hline WVREF627-551 & 29376 & WVREF627 & $8 / 2 / 0814: 24$ & 0 & 551 \\
\hline WVREF627-552 & 29383 & WVREF627 & $8 / 2 / 08$ 14:30 & 0 & 552 \\
\hline WVREF627-553 & 29394 & WVREF627 & $8 / 2 / 08$ 14:40 & -0.0002 & 553 \\
\hline WVREF627-554 & 29395 & WVREF627 & $8 / 2 / 08$ 14:42 & 0 & 554 \\
\hline WVREF627-555 & 29406 & WVREF627 & $8 / 2 / 08$ 14:52 & 0.0008 & 555 \\
\hline WVREF627-556 & 29407 & WVREF627 & $8 / 2 / 08$ 14:53 & 0 & 556 \\
\hline WVREF627-557 & 29410 & WVREF627 & $8 / 2 / 08$ 14:55 & 0 & 557 \\
\hline WVREF627-558 & 29411 & WVREF627 & $8 / 3 / 08$ 12:20 & 0.0034 & 558 \\
\hline WVREF627-559 & 29412 & WVREF627 & $8 / 3 / 08$ 12:25 & 0 & 559 \\
\hline WVREF627-560 & 29414 & WVREF627 & $8 / 3 / 08$ 12:27 & -0.0002 & 560 \\
\hline WVREF627-561 & 29415 & WVREF627 & $8 / 3 / 08$ 12:27 & 0 & 561 \\
\hline WVREF627-562 & 29421 & WVREF627 & $8 / 3 / 08$ 12:31 & -0.0003 & 562 \\
\hline WVREF627-563 & 29422 & WVREF627 & $8 / 3 / 08$ 12:31 & 0 & 563 \\
\hline WVREF627-564 & 29428 & WVREF627 & $8 / 3 / 0812: 36$ & -0.0004 & 564 \\
\hline WVREF627-565 & 29429 & WVREF627 & $8 / 3 / 08$ 12:37 & 0 & 565 \\
\hline WVREF627-566 & 29440 & WVREF627 & $8 / 3 / 08$ 12:44 & -0.0004 & 566 \\
\hline WVREF627-567 & 29441 & WVREF627 & $8 / 3 / 08$ 12:46 & 0 & 567 \\
\hline WVREF627-568 & 29452 & WVREF627 & $8 / 3 / 08$ 12:54 & 0.0005 & 568 \\
\hline WVREF627-569 & 29453 & WVREF627 & $8 / 3 / 0812: 55$ & 0 & 569 \\
\hline WVREF627-570 & 29456 & WVREF627 & $8 / 3 / 08$ 12:57 & 0.0006 & 570 \\
\hline WVREF627-571 & 29457 & WVREF627 & $8 / 4 / 08$ 12:39 & 0.0008 & 571 \\
\hline WVREF627-572 & 29458 & WVREF627 & $8 / 4 / 08$ 12:50 & 0 & 572 \\
\hline WVREF627-573 & 29460 & WVREF627 & $8 / 4 / 08$ 12:52 & 0.0008 & 573 \\
\hline WVREF627-574 & 29461 & WVREF627 & $8 / 4 / 08$ 12:52 & 0 & 574 \\
\hline WVREF627-575 & 29466 & WVREF627 & $8 / 4 / 08$ 12:58 & -0.0004 & 575 \\
\hline WVREF627-576 & 29467 & WVREF627 & $8 / 4 / 0813: 00$ & 0 & 576 \\
\hline WVREF627-577 & 29478 & WVREF627 & 8/4/08 13:08 & 0.0015 & 577 \\
\hline WVREF627-578 & 29479 & WVREF627 & 8/4/08 13:13 & 0 & 578 \\
\hline WVREF627-579 & 29486 & WVREF627 & $8 / 4 / 08$ 13:20 & -0.0003 & 579 \\
\hline WVREF627-580 & 29504 & WVREF627 & $8 / 5 / 08$ 12:49 & -0.0029 & 580 \\
\hline WVREF627-581 & 29505 & WVREF627 & $8 / 5 / 0812: 54$ & 0 & 581 \\
\hline WVREF627-582 & 29507 & WVREF627 & $8 / 5 / 0812: 55$ & 0.0001 & 582 \\
\hline WVREF627-583 & 29508 & WVREF627 & $8 / 5 / 0812: 55$ & 0 & 583 \\
\hline WVREF627-584 & 29513 & WVREF627 & $8 / 5 / 0812: 58$ & -0.0006 & 584 \\
\hline WVREF627-585 & 29514 & WVREF627 & $8 / 5 / 08$ 12:59 & 0 & 585 \\
\hline WVREF627-586 & 29521 & WVREF627 & 8/5/08 13:03 & -0.0001 & 586 \\
\hline WVREF627-587 & 29548 & WVREF627 & $8 / 7 / 08$ 17:15 & -0.0008 & 587 \\
\hline WVREF627-588 & 29549 & WVREF627 & $8 / 7 / 08$ 17:15 & 0 & 588 \\
\hline WVREF627-589 & 29550 & WVREF627 & 8/7/08 17:16 & 0 & 589 \\
\hline WVREF627-590 & 29555 & WVREF627 & $8 / 7 / 0817: 21$ & 0 & 590 \\
\hline
\end{tabular}




\begin{tabular}{|c|c|c|c|c|}
\hline WVREF627-591 & 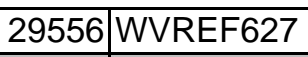 & 8/7/08 17:21 & 0 & 591 \\
\hline WVREF627-592 & 29567|WVREF627 & 8/7/08 17:34 & -0.0004 & 592 \\
\hline WVREF627-593 & \begin{tabular}{|l|l|} 
29568 & WVEF627
\end{tabular} & $8 / 7 / 08$ 17:34 & 0 & 593 \\
\hline WVREF627-594 & \begin{tabular}{l|l}
29579 & WVREF627
\end{tabular} & $8 / 7 / 0817: 46$ & -0.0004 & 594 \\
\hline WVREF627-595 & 29580 WVREF627 & $8 / 7 / 08$ 17:46 & 0 & 595 \\
\hline WVREF627-596 & 29591 WVREF627 & 8/7/08 17:58 & 0.0011 & 596 \\
\hline WVREF627-597 & \begin{tabular}{|l|l|}
29592 & WVREF627 \\
\end{tabular} & 8/7/08 17:58 & 0 & 597 \\
\hline WVREF627-598 & 29603 WVREF627 & 8/7/08 18:51 & -0.0003 & 598 \\
\hline WVREF627-599 & 29604|WVREF627 & 8/7/08 18:51 & 0 & 599 \\
\hline WVREF627-600 & 29615 WVREF627 & 8/7/08 19:01 & -0.0003 & 600 \\
\hline WVREF627-601 & \begin{tabular}{|l|l|}
29616 & WVREF627 \\
\end{tabular} & 8/7/08 19:01 & 0 & 601 \\
\hline WVREF627-602 & 29627 WVREF627 & $8 / 7 / 08$ 19:10 & 0.0002 & 602 \\
\hline WVREF627-603 & \begin{tabular}{|l|l|}
29628 & WVREF627 \\
\end{tabular} & 8/7/08 19:10 & 0 & 603 \\
\hline WVREF627-604 & 29639 WVREF627 & 8/7/08 19:21 & -0.0006 & 604 \\
\hline WVREF627-605 & \begin{tabular}{|l|l|}
29640 & WVREF627 \\
\end{tabular} & 8/7/08 19:21 & 0 & 605 \\
\hline WVREF627-606 & 29651 WVREF627 & 8/7/08 19:30 & -0.0006 & 606 \\
\hline WVREF627-607 & \begin{tabular}{|l|l|}
29652 & WVREF627 \\
\end{tabular} & 8/7/08 19:30 & 0 & 607 \\
\hline WVREF627-608 & 29653 WVREF627 & $8 / 7 / 08$ 19:31 & 0 & 608 \\
\hline WVREF627-609 & 29664 WVREF627 & 8/7/08 19:39 & 0.0008 & 609 \\
\hline WVREF627-610 & 29665 WVREF627 & $8 / 7 / 08$ 19:40 & 0 & 610 \\
\hline WVREF627-611 & 29676 WVREF627 & 8/7/08 19:48 & 0.0007 & 611 \\
\hline WVREF627-612 & \begin{tabular}{l|l|}
29677 & WVREF627 \\
\end{tabular} & $8 / 7 / 08$ 19:49 & 0 & 612 \\
\hline WVREF627-613 & \begin{tabular}{|l|l|}
29688 & WVREF627 \\
\end{tabular} & 8/7/08 19:57 & 0.0005 & 613 \\
\hline WVREF627-614 & 29689 WVREF627 & 8/7/08 19:58 & 0 & 614 \\
\hline WVREF627-615 & 29700 WVREF627 & 8/7/08 20:06 & 0.0013 & 615 \\
\hline WVREF627-616 & \begin{tabular}{l|l}
29701 & WVREF627 \\
\end{tabular} & $8 / 7 / 0820: 06$ & 0 & 616 \\
\hline WVREF627-617 & \begin{tabular}{|l|l|}
29712 & WVREF627 \\
\end{tabular} & 8/7/08 20:14 & 0.0005 & 617 \\
\hline WVREF627-618 & 29713 WVREF627 & $8 / 7 / 08$ 20:14 & 0 & 618 \\
\hline WVREF627-619 & 29724 WVREF627 & 8/7/08 20:22 & -0.0002 & 619 \\
\hline WVREF627-620 & 29725 WVREF627 & $8 / 7 / 08$ 20:23 & 0 & 620 \\
\hline WVREF627-621 & 29732|WVREF627 & 8/7/08 20:32 & 0.0002 & 621 \\
\hline WVREF627-622 & 29733 WVREF627 & 8/7/08 20:33 & 0 & 622 \\
\hline WVREF627-623 & 29739 WVREF627 & $8 / 7 / 0820: 37$ & 0.0014 & 623 \\
\hline WVREF627-624 & \begin{tabular}{l|l}
29837 & WVREF627 \\
\end{tabular} & 8/14/08 15:29 & -0.0015 & 624 \\
\hline WVREF627-625 & \begin{tabular}{|l|l|}
29838 & WVREF627 \\
\end{tabular} & $8 / 14 / 08$ 15:32 & 0 & 625 \\
\hline WVREF627-626 & 29839 WVREF627 & $8 / 14 / 08$ 15:34 & 0 & 626 \\
\hline WVREF627-627 & 29844 WVREF627 & $8 / 14 / 08$ 15:39 & -0.0013 & 627 \\
\hline WVREF627-628 & 29845 WVREF627 & 8/14/08 15:39 & 0 & 628 \\
\hline WVREF627-629 & \begin{tabular}{|l|l|}
29856 & WVREF627 \\
\end{tabular} & 8/14/08 15:48 & -0.0001 & 629 \\
\hline WVREF627-630 & 29857 WVREF627 & 8/14/08 15:49 & 0 & 630 \\
\hline WVREF627-631 & \begin{tabular}{|l|l|}
29868 & WVREF627 \\
\end{tabular} & $8 / 14 / 08$ 15:56 & -0.001 & 631 \\
\hline WVREF627-632 & 29869 WVREF627 & 8/14/08 15:57 & 0 & 632 \\
\hline WVREF627-633 & \begin{tabular}{|l|l|}
29880 & WVREF627 \\
\end{tabular} & 8/14/08 16:05 & -0.0008 & 633 \\
\hline WVREF627-634 & 29881 WVREF627 & 8/14/08 16:05 & 0 & 634 \\
\hline WVREF627-635 & \begin{tabular}{|l|l|}
29892 & WVREF627 \\
\end{tabular} & $8 / 14 / 08$ 16:15 & 0.0014 & 635 \\
\hline WVREF627-636 & 29893 WVREF627 & 8/14/08 16:17 & 0 & 636 \\
\hline WVREF627-637 & \begin{tabular}{l|l}
29904 & WVREF627 \\
\end{tabular} & $8 / 14 / 08$ 16:26 & 0.0009 & 637 \\
\hline WVREF627-638 & 29905 WVREF627 & 8/14/08 16:26 & 0 & 638 \\
\hline WVREF627-639 & \begin{tabular}{l|l}
29916 & WVREF627 \\
\end{tabular} & 8/14/08 16:34 & -0.0002 & 639 \\
\hline
\end{tabular}




\begin{tabular}{|c|c|c|c|c|}
\hline WVREF627-640 & 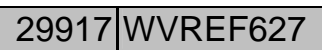 & 8/14/08 16:34 & 0 & 640 \\
\hline WVREF627-641 & 29928|WVREF627 & $8 / 14 / 08$ 16:42 & -0.0001 & 641 \\
\hline WVREF627-642 & 29929 WVREF627 & 8/14/08 16:42 & 0 & 642 \\
\hline WVREF627-643 & 29940|WVREF627 & 8/14/08 16:49 & -0.0001 & 643 \\
\hline WVREF627-644 & \begin{tabular}{l|l|l}
30005 & WVREF627
\end{tabular} & 8/21/08 9:36 & -0.0006 & 644 \\
\hline WVREF627-645 & \begin{tabular}{l|l|l}
30006 & WVREF627
\end{tabular} & 8/21/08 9:41 & 0 & 645 \\
\hline WVREF627-646 & \begin{tabular}{l|l|l}
30008 & WVREF627
\end{tabular} & 8/21/08 9:42 & 0.0006 & 646 \\
\hline WVREF627-647 & \begin{tabular}{l|l|l}
30009 & WVREF627
\end{tabular} & 8/21/08 9:42 & 0 & 647 \\
\hline WVREF627-648 & 30014 WVREF627 & 8/21/08 9:47 & -0.0027 & 648 \\
\hline WVREF627-649 & 30015|WVREF627 & $8 / 21 / 089: 47$ & 0 & 649 \\
\hline WVREF627-650 & \begin{tabular}{l|l|l}
30026 & WVREF627
\end{tabular} & 8/21/08 9:56 & -0.0004 & 650 \\
\hline WVREF627-651 & \begin{tabular}{l|l|l}
30027 & WVREF627
\end{tabular} & 8/21/08 9:56 & 0 & 651 \\
\hline WVREF627-652 & \begin{tabular}{|l|l|}
30038 & WVREF627 \\
\end{tabular} & $8 / 21 / 08$ 10:05 & -0.0007 & 652 \\
\hline WVREF627-653 & 30039 WVREF627 & $8 / 21 / 08$ 10:05 & 0 & 653 \\
\hline WVREF627-654 & 30050|WVREF627 & 8/21/08 10:13 & 0.0002 & 654 \\
\hline WVREF627-655 & 30051 $\mid$ WVREF627 & $8 / 21 / 08$ 10:14 & 0 & 655 \\
\hline WVREF627-656 & \begin{tabular}{l|l|l}
30062 & WVREF627 \\
\end{tabular} & $8 / 21 / 08$ 10:25 & 0.0012 & 656 \\
\hline WVREF627-657 & 30063|WVREF627 & $8 / 21 / 08$ 10:25 & 0 & 657 \\
\hline WVREF627-658 & 30074|WVREF627 & $8 / 21 / 08$ 10:34 & 0.0006 & 658 \\
\hline WVREF627-659 & 30075|WVREF627 & $8 / 21 / 08$ 10:34 & 0 & 659 \\
\hline WVREF627-660 & \begin{tabular}{|l|l|}
30086 & WVREF627 \\
\end{tabular} & 8/21/08 10:43 & 0.0006 & 660 \\
\hline WVREF627-661 & \begin{tabular}{l|l|l|}
30087 & WVREF627 \\
\end{tabular} & $8 / 21 / 08$ 10:43 & 0 & 661 \\
\hline WVREF627-662 & 30098 $\mid$ WVREF627 & $8 / 21 / 08$ 10:53 & -0.001 & 662 \\
\hline WVREF627-663 & 30099 WVREF627 & $8 / 21 / 08$ 10:54 & 0 & 663 \\
\hline WVREF627-664 & \begin{tabular}{l|l|l}
30100 & WVREF627
\end{tabular} & $8 / 21 / 08$ 10:54 & 0 & 664 \\
\hline WVREF627-665 & \begin{tabular}{l|l}
30111 & WVREF627 \\
\end{tabular} & 8/21/08 11:02 & 0.0007 & 665 \\
\hline WVREF627-666 & \begin{tabular}{l|l|l}
30260 & WVREF627 \\
\end{tabular} & 8/28/08 9:14 & -0.0019 & 666 \\
\hline WVREF627-667 & \begin{tabular}{l|l|l}
30261 & WVREF627
\end{tabular} & 8/28/08 9:18 & 0 & 667 \\
\hline WVREF627-668 & \begin{tabular}{|l|l|}
30263 & WVREF627 \\
\end{tabular} & 8/28/08 9:19 & 0.0005 & 668 \\
\hline WVREF627-669 & 30264 $\mid$ WVREF627 & 8/28/08 9:20 & 0 & 669 \\
\hline WVREF627-670 & \begin{tabular}{l|l|l|}
30269 & WVREF627 \\
\end{tabular} & 8/28/08 9:24 & 0.0007 & 670 \\
\hline WVREF627-671 & \begin{tabular}{l|l|l}
30270 & WVREF627
\end{tabular} & 8/28/08 9:25 & 0 & 671 \\
\hline WVREF627-672 & \begin{tabular}{l|l}
30281 & WVREF627 \\
\end{tabular} & 8/28/08 9:33 & -0.0006 & 672 \\
\hline WVREF627-673 & \begin{tabular}{l|l}
30282 & WVREF627
\end{tabular} & 8/28/08 9:34 & 0 & 673 \\
\hline WVREF627-674 & \begin{tabular}{|l|l|}
30293 & WVREF627 \\
\end{tabular} & 8/28/08 9:42 & 0.0007 & 674 \\
\hline WVREF627-675 & \begin{tabular}{l|l}
30294 & WVREF627 \\
\end{tabular} & 8/28/08 9:42 & 0 & 675 \\
\hline WVREF627-676 & \begin{tabular}{l|l|l|}
30305 & WVREF627 \\
\end{tabular} & 8/28/08 9:51 & 0.0005 & 676 \\
\hline WVREF627-677 & \begin{tabular}{|l|l|}
30306 & WVREF627 \\
\end{tabular} & 8/28/08 9:52 & 0 & 677 \\
\hline WVREF627-678 & 30317 WVREF627 & $8 / 28 / 08$ 10:00 & 0.0001 & 678 \\
\hline WVREF627-679 & 30318 WVREF627 & 8/28/08 10:01 & 0 & 679 \\
\hline WVREF627-680 & \begin{tabular}{|l|l|}
30329 & WVREF627 \\
\end{tabular} & 8/28/08 10:10 & 0.0008 & 680 \\
\hline WVREF627-681 & \begin{tabular}{l|l|l}
30330 & WVREF627 \\
\end{tabular} & 8/28/08 10:11 & 0 & 681 \\
\hline WVREF627-682 & 30341 WVREF627 & $8 / 28 / 08$ 10:20 & 0.0008 & 682 \\
\hline WVREF627-683 & \begin{tabular}{l|l|l}
30342 & WVREF627 \\
\end{tabular} & $8 / 28 / 08$ 10:20 & 0 & 683 \\
\hline WVREF627-684 & 30353 WVREF627 & $8 / 28 / 08$ 10:29 & -0.0009 & 684 \\
\hline WVREF627-685 & 30354 $\mid$ WVREF627 & $8 / 28 / 08$ 10:30 & 0 & 685 \\
\hline WVREF627-686 & \begin{tabular}{l|l|}
30365 & WVREF627 \\
\end{tabular} & $8 / 28 / 08$ 10:40 & 0.0007 & 686 \\
\hline WVREF627-687 & \begin{tabular}{l|l|l}
30481 & WVREF627 \\
\end{tabular} & 9/3/08 14:14 & -0.0109 & 687 \\
\hline WVREF627-688 & \begin{tabular}{l|l|l}
30482 & WVREF627 \\
\end{tabular} & 9/3/08 14:22 & 0 & 688 \\
\hline
\end{tabular}




\begin{tabular}{|l|r|l|r|r|r|}
\hline WVREF627-689 & 30484 & WVREF627 & $9 / 3 / 0814: 23$ & -0.0002 & 689 \\
\hline WVREF627-690 & 30485 & WVREF627 & $9 / 3 / 0814: 24$ & 0 & 690 \\
\hline WVREF627-691 & 30490 & WVREF627 & $9 / 3 / 0814: 28$ & 0.0001 & 691 \\
\hline WVREF627-692 & 30491 & WVREF627 & $9 / 3 / 0814: 29$ & 0 & 692 \\
\hline WVREF627-693 & 30502 & WVREF627 & $9 / 3 / 0814: 37$ & -0.0003 & 693 \\
\hline WVREF627-694 & 30503 & WVREF627 & $9 / 3 / 0814: 39$ & 0 & 694 \\
\hline WVREF627-695 & 30514 & WVREF627 & $9 / 3 / 0814: 49$ & -0.0006 & 695 \\
\hline WVREF627-696 & 30515 & WVREF627 & $9 / 3 / 0814: 52$ & 0 & 696 \\
\hline WVREF627-697 & 30526 & WVREF627 & $9 / 3 / 0815: 03$ & 0.0006 & 697 \\
\hline WVREF627-698 & 30527 & WVREF627 & $9 / 3 / 0815: 03$ & 0 & 698 \\
\hline WVREF627-699 & 30538 & WVREF627 & $9 / 3 / 0815: 15$ & -0.0011 & 699 \\
\hline WVREF627-700 & 30539 & WVREF627 & $9 / 3 / 0815: 17$ & 0 & 700 \\
\hline WVREF627-701 & 30550 & WVREF627 & $9 / 3 / 0815: 26$ & 0.0004 & 701 \\
\hline WVREF627-702 & 30551 & WVREF627 & $9 / 3 / 0815: 50$ & 0.0018 & 702 \\
\hline WVREF627-703 & 30552 & WVREF627 & $9 / 3 / 0816: 35$ & 0 & 703 \\
\hline WVREF627-704 & 30563 & WVREF627 & $9 / 3 / 0816: 44$ & 0.0014 & 704 \\
\hline WVREF627-705 & 30564 & WVREF627 & $9 / 3 / 0816: 46$ & 0 & 705 \\
\hline WVREF627-706 & 30575 & WVREF627 & $9 / 3 / 0816: 55$ & 0.0002 & 706 \\
\hline WVREF627-707 & 30576 & WVREF627 & $9 / 3 / 0816: 55$ & 0 & 707 \\
\hline WVREF627-708 & 30587 & WVREF627 & $9 / 3 / 0817: 06$ & -0.001 & 708 \\
\hline
\end{tabular}

